\title{
Structural and functional investigation of cargo recognition by exportins
}

\author{
Dissertation \\ in partial fulfillment of the requirements \\ for the degree \\ "Doctor rerum naturalium (Dr. rer. nat)" \\ in the Molecular Biology Program \\ at the Georg-August University Göttingen, \\ Faculty of Biology
}

\author{
submitted by \\ Metin Aksu \\ born in \\ Çorum, Turkey
}

Göttingen, September 2015 
Members of the Thesis Committee

Prof. Dr. Dirk Görlich

(Supervisor and Referee)

Prof. Dr. Reinhard Lührmann

(Co-referee)

Prof. Dr. Peter Rehling
Department of Cellular Logistics

Max Planck Institute for Biophysical Chemistry

Göttingen, Germany

Department of Cellular Biochemistry

Max Planck Institute for Biophysical Chemistry

Göttingen, Germany

Department of Cellular Biochemistry

Georg August University Göttingen

Göttingen, Germany

\section{Additional Members of the Examination Board}

Prof. Dr. Patrick Cramer

Prof. Dr. Holger Stark

Prof. Dr. Henning Urlaub
Department of Molecular Biology

Max Planck Institute for Biophysical Chemistry

Göttingen, Germany

Department of Structural Dynamics

Max Planck Institute for Biophysical Chemistry

Göttingen, Germany

Bioanalytical Mass Spectrometry Research Group

Max Planck Institute for Biophysical Chemistry

Göttingen, Germany

Date of oral examination November 17, 2015 
To those I love the most 


\section{ACKNOWLEDGEMENTS}

First of all, I thank my supervisor Dirk Görlich for the opportunity to work with him, his input for this work and his continuous support throughout my PhD. His scientific knowledge and enthusiasm always inspired me.

I would like to thank Reinhard Lührmann and Peter Rehling for being part of my thesis committee and their valuable comments and suggestions for the project. I thank Patrick Cramer, Holger Stark and Henning Urlaub for joining my extended thesis committee.

Crystallization was at the heart of this project and most of the work was done at the crystallization facility of our institute. I thank Vladimir Pena and Jürgen Wawrzinek for establishing the facility, Jürgen Wawrzinek for excellent technical support and Ulrich Steurwald for discussions and suggestions about crystallography.

I sincerely thank Sergei Trakhanov for sharing his crystallographic knowledge with me, his contribution to the structure solution and the great time at the synchrotron.

I thank Henning Urlaub, Samir Karaca, Monika Raabe and Uwe Plessmann for the mass spectrometric analysis, Miroslav Nikolov for kindly providing the SILAC HeLa extracts.

I would like to thank Dirk Görlich, Koray Kirli, Avani Shukla, Rüstem Yilmaz and Ömer Çiçek for the critical reading of the various parts of this thesis.

I am also thankful to Steffen Burkhardt, Kerstin Grüniger and Cornelia Paz for administrative support.

Additional thanks go to:

Renate, Heinz-Jürgen, Jens and Susanne for technical assistance.

Gabi and Uwe for taking care of many things and making this lab a ready to use place. Without their contribution, this project would take much longer.

Jenny and Connie for christmas cookies, Heinz-Jürgen for delicious cakes.

Bastian, Koray and Kevser for scientific and non-scientific discussions during coffee breaks

All the former and current members of the lab for lovely conversations during breaks, fruitful discussions during the seminars and the great atmosphere in the lab.

Koray, Kevser, Sinem, Samir, Ömer and the other Turkish community for their friendship, all the fun and not letting me get homesick easily. 
Avani, Jonas, Maria, Ömer, Sona, Ingrid and Myro for reminding me that there is a life outside the lab. All the parties, game nights and other outings were much better when they were with me.

Rüstem for his continuous support and friendship.

Last but not least, I would like to thank my mother and sister for supporting and motivating me all the time. 


\section{TABLE OF CONTENTS}

ACKNOWLEDGEMENTS ........................................................................................ vii

TABLE OF CONTENTS .......................................................................................

LIST OF FIGURES ............................................................................................. xiii

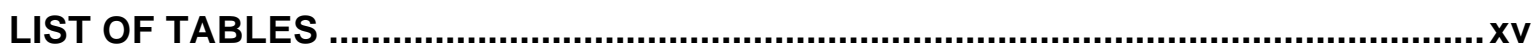

LIST OF ABBREVIATIONS .......................................................................................xi

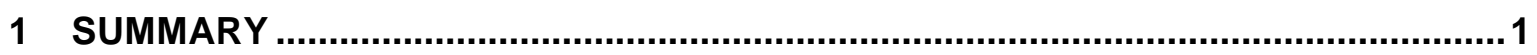

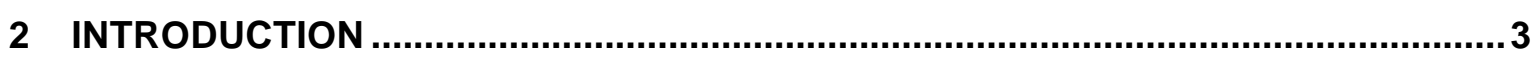

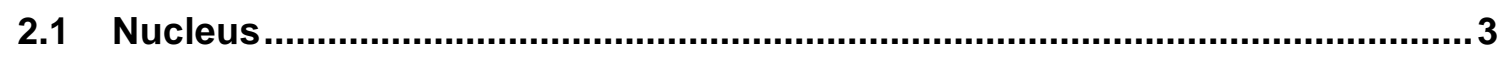

2.2 Nucleocytoplasmic Transport ................................................................ 4

2.2.1 Importin $\beta$-like nuclear transport receptors ............................................. 4

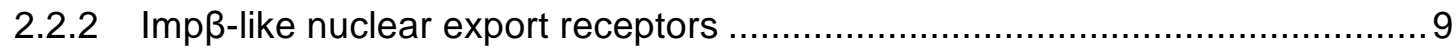

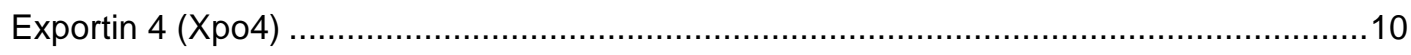

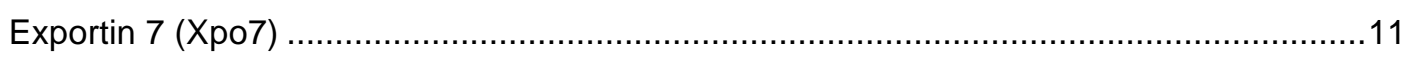

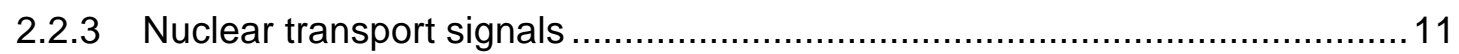

2.3 The structural features of Imp $\beta$-like NTRs ..................................................13

2.3.1 The regulator of NTR •cargo interaction: Ran.......................................... 15

2.3.2 Interaction of RanGTP with Impß-like NTRs .............................................. 16

2.3.3 Cargo recognition by Imp $\beta$-like nuclear export receptors ............................. 19

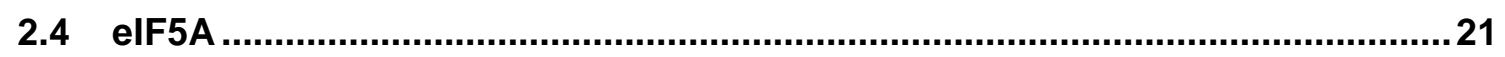

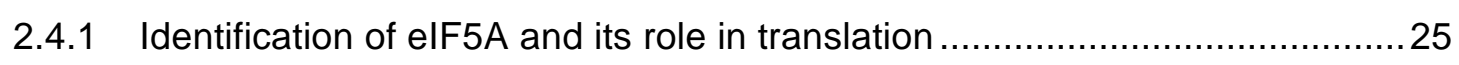

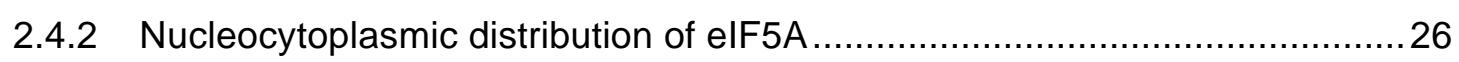

2.5 About this work.................................................................................

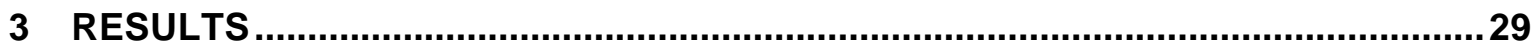

3.1 Structural and Biochemical Analysis of Cargo Recognition by Xpo4...........29

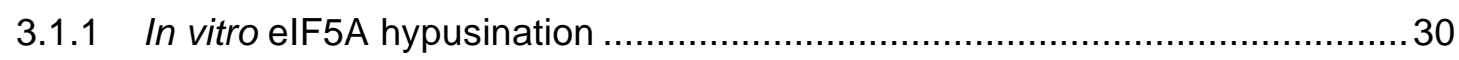

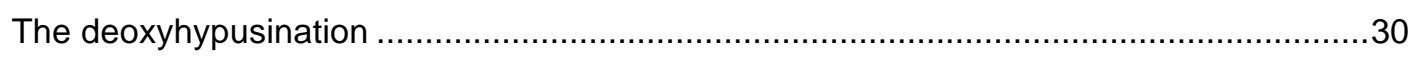

The reversal of the deoxyhypusination and the hydroxylation .........................................31

Assessing the effect of the modifications on Xpo4 binding …………...............................34

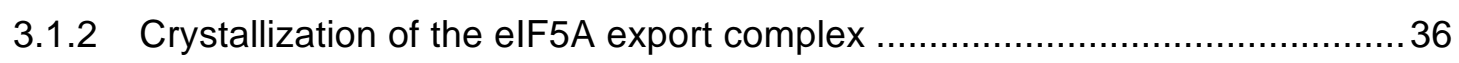

Reconstitution of elF5A export complex .....................................................................

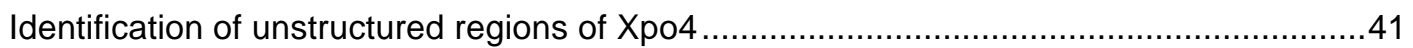

Generation of loop deletions and crystallization...........................................................50

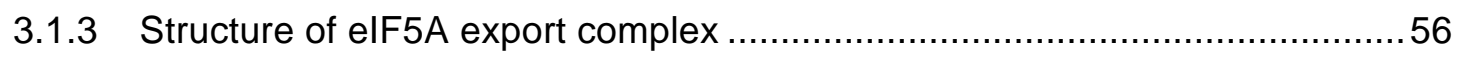




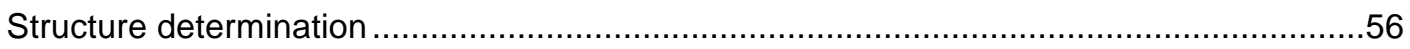

Overall structure of Xpo4

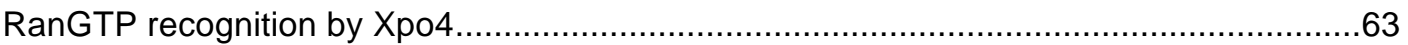

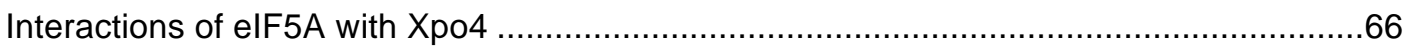

Analysis of the structure with biochemical and functional assays ....................................69

3.1.4 Crystallization trials of the Sox2 import complex ........................................ 76

3.1.5 Crystallization trials of Xpo4 and Xpo4-RanGTP complex ........................... 82

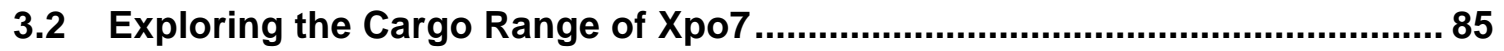

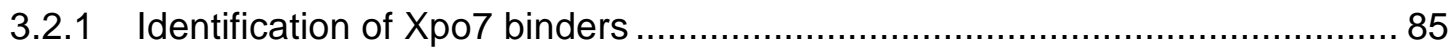

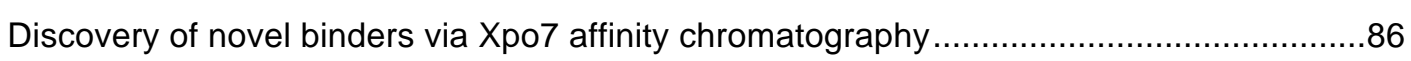

3.2.2 Validation of the interaction of the selected binders with Xpo7 …................. 92

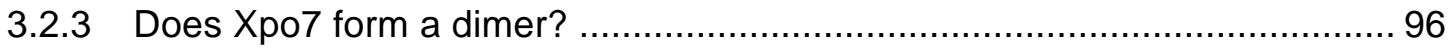

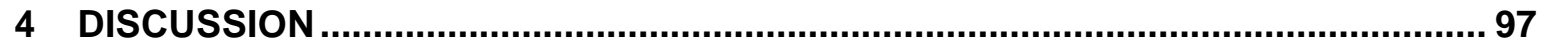

4.1 Xpo4 mediated nucleocytoplasmic transport.................................................... 97

4.1.1 Implications for cargo loading and cargo release mechanisms.................. 100

4.1.2 Implications for Xpo4 function ............................................................ 102

4.1.3 Implications for elF5A function ............................................................. 103

4.1.4 How does Xpo4 recognize other cargoes? ............................................... 104

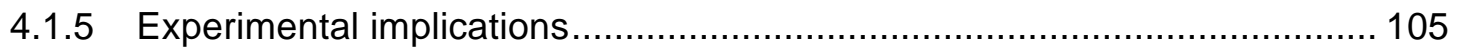

In vitro hypusine-containing elF5A production ..........................................................106

4.2 What have we learned about Xpo7? .................................................................. 107

4.2.1 Xpo7 is a broad-spectrum exportin .................................................... 107

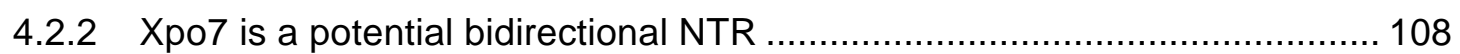

4.2.3 Functional significance of dimeric Xpo7 .................................................... 109

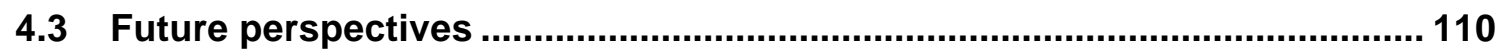

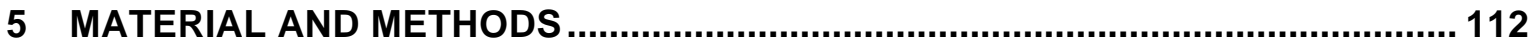

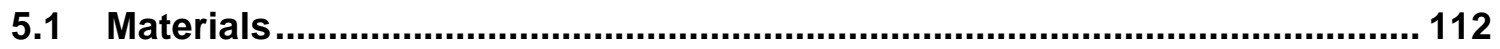

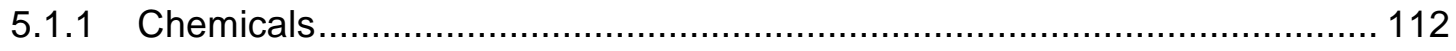

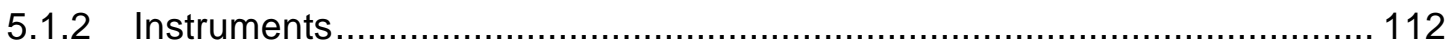

5.1.3 Software and bioinformatics tools ......................................................... 113

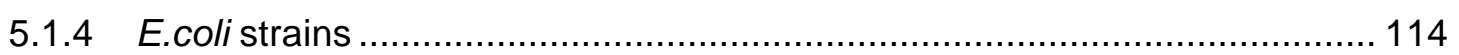

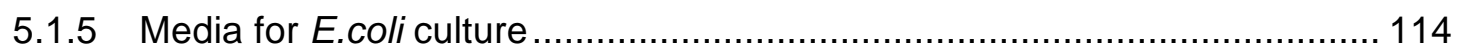

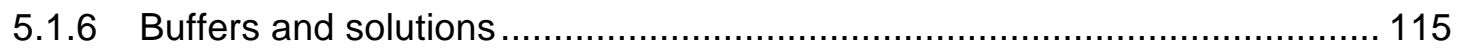

5.1.7 Commercial crystallization screens ..................................................... 116

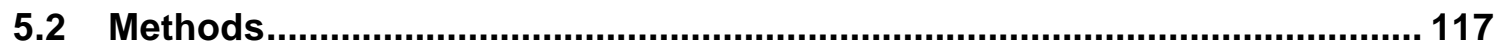

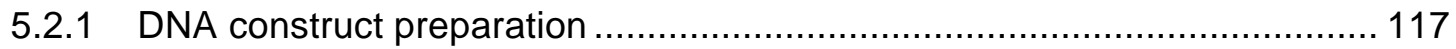

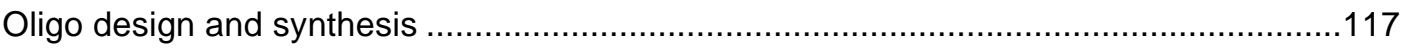




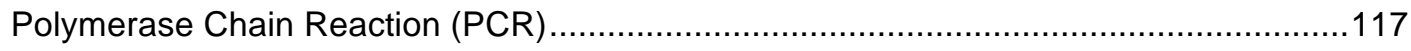

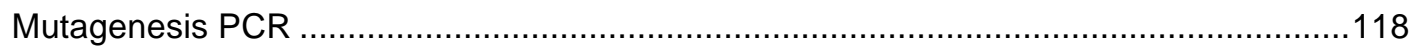

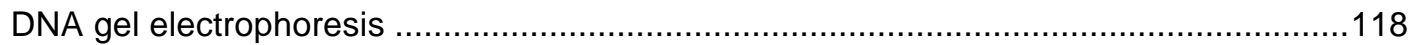

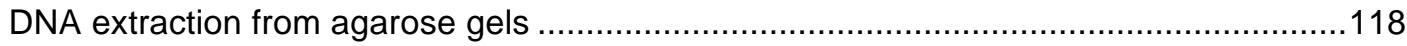

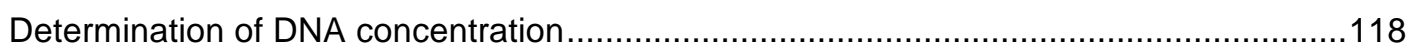

DNA cleavage with restriction enzymes ............................................................ 118

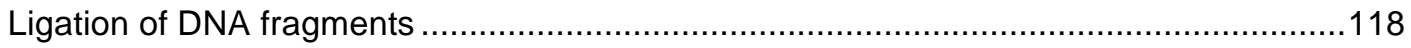

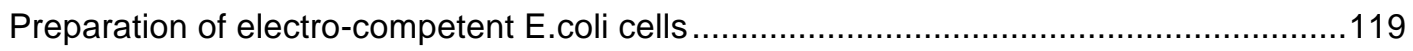

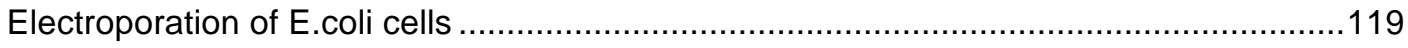

DNA purification from E.coli cultures.................................................................120

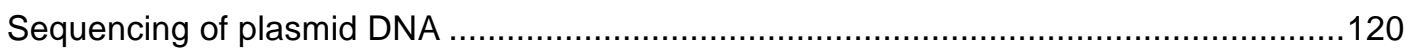

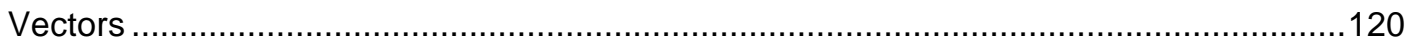

5.2.2 Protein expression and purification........................................................ 122

Expression and purification of Xpo4 variants ....................................................122

Expression and purification of Selenomethionine-substituted Xpo4 ............................123

Expression and purification of RanGTP ............................................................... 124

Expression and purification of other proteins ................................................. 125

Expression of proteins with in vivo biotinylation ............................................. 125

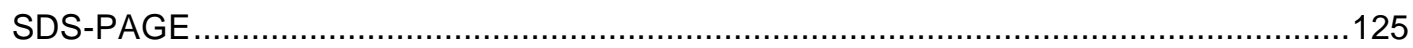

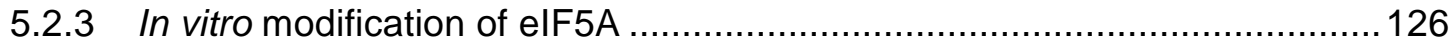

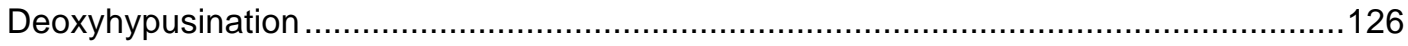

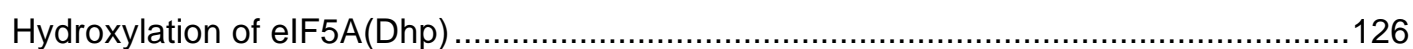

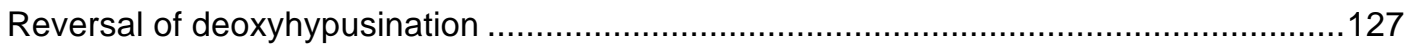

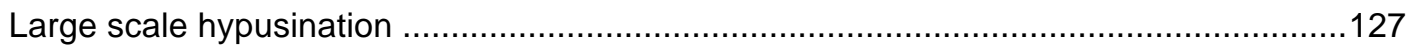

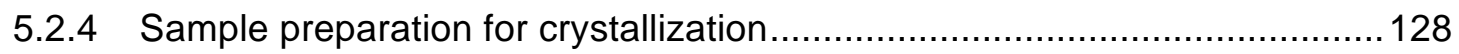

Reconstitution of the elF5A export complexes......................................................128

Reconstitution of the RanGTP•Xpo4 complex ................................................129

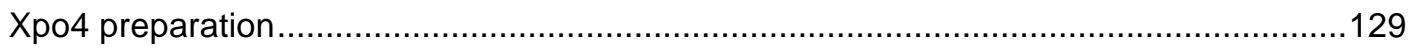

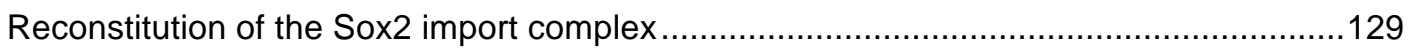

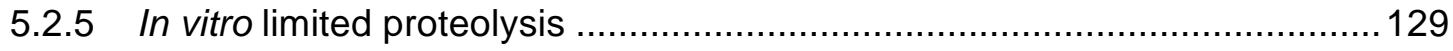

5.2.6 Molecular weight determination with static light scattering (SLS) ............. 130

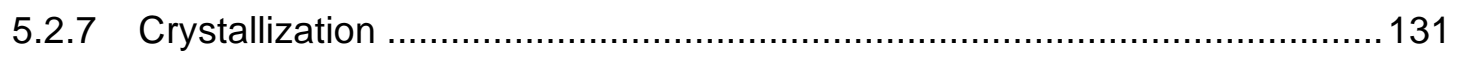

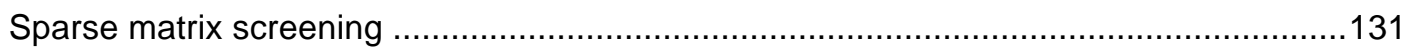

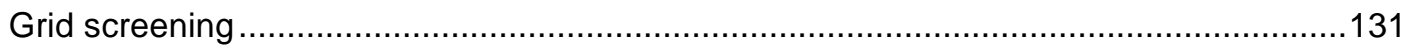

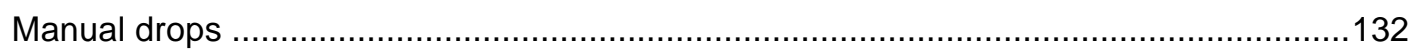

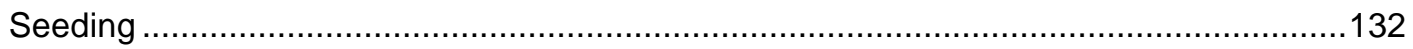

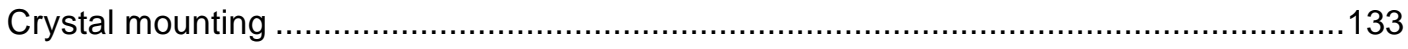

In situ limited proteolysis ................................................................................ 133

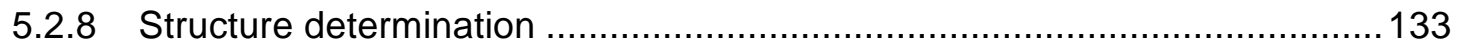

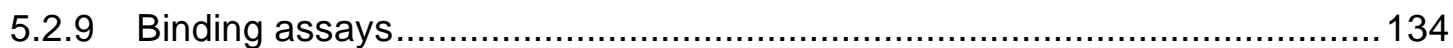


Binding assays with anti-Z affibody dimer beads 135

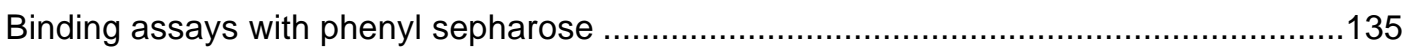

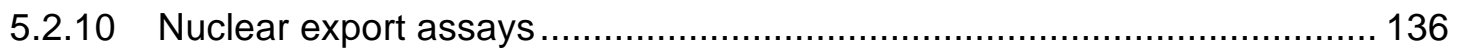

5.2.11 Pull down experiments from cytoplasmic Hela extracts .......................... 136

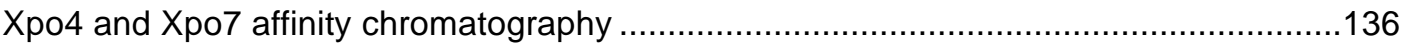

Affinity chromatography with Xpo7 cargoes …….....................................................137

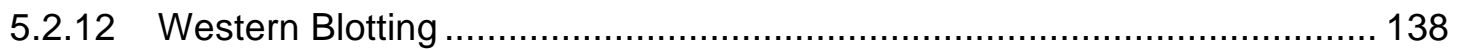

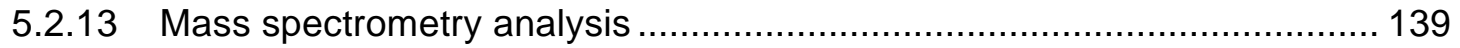

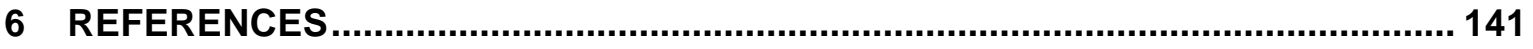

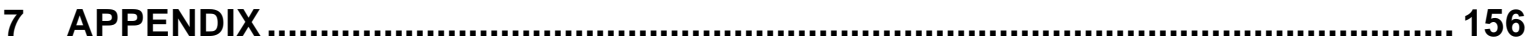




\section{LIST OF FIGURES}

Figure 2-1 Overview of the nucleocytoplasmic transport cycles................................... 6

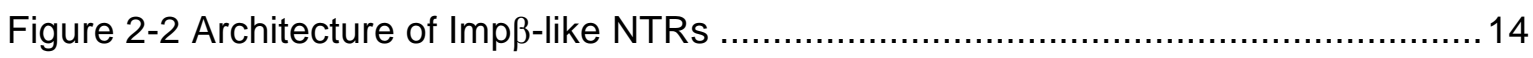

Figure 2-3 Comparison of GDP- and GTP-bound structures of Ran ............................... 16

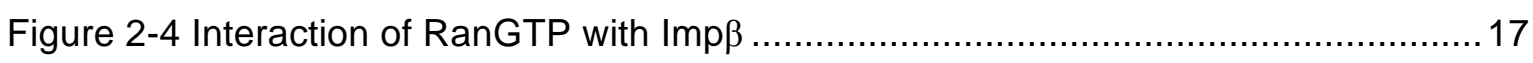

Figure 2-5 Interaction of exportins with RanGTP and respective cargoes ....................... 18

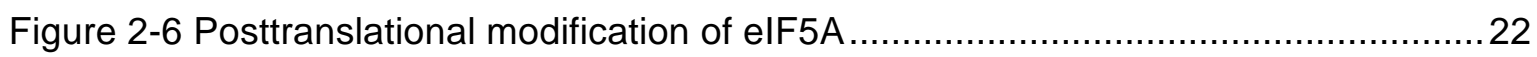

Figure 2-7 Multiple sequence alignment of elF5A from different species.......................... 23

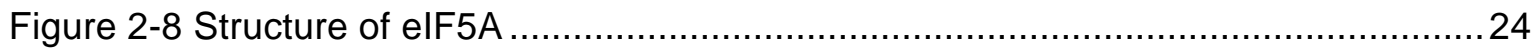

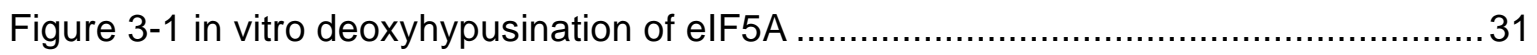

Figure 3-2 The reversal of the deoxyhypusination assays with elF5A(Dhp) and

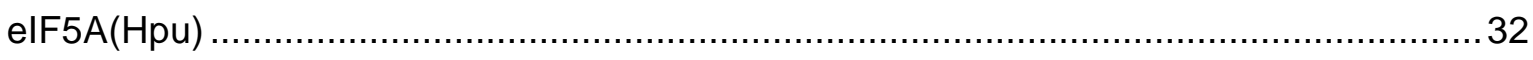

Figure 3-3 Scheme for large scale elF5A(Hpu) purification ........................................... 34

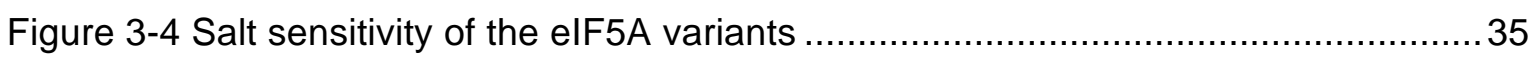

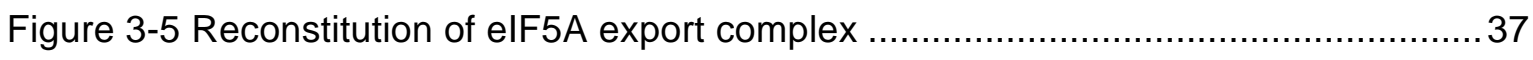

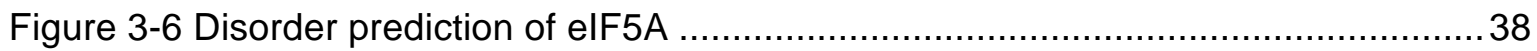

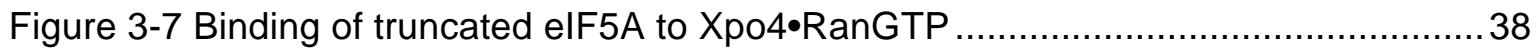

Figure 3-8 Reconstitution of elF5A15-154 export complex and the crystallization hits ..... 41

Figure 3-9 in vitro limited proteolysis of elF5A export complexes ................................ 42

Figure 3-10 Identification of the protease cleavage sites ............................................ 44

Figure 3-11 Crystallization drops of in situ limited proteolysis ....................................... 46

Figure 3-12 SDS-PAGE analysis of the trypsin and chymotrypsin digested crystals ........ 47

Figure 3-13 in vitro limited proteolysis with trypsin coupled to size exclusion chromatography.

Figure 3-14 in vitro limited proteolysis with chymotrypsin coupled to size exclusion chromatography 49

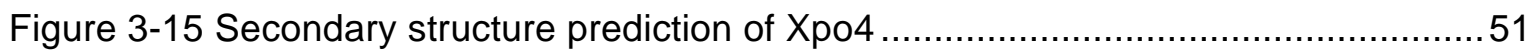

Figure 3-16 Sequence alignment of Xpo4 homologues ............................................. 52

Figure 3-17 Schematic representation of the Xpo4 loop mutants ....................................53

Figure 3-18 elF5A export complex formation with Xpo4 loop mutants..............................54

Figure 3-19 Crystallization of the export complexes consisting Xpo4 loop mutants..........55

Figure 3-20 Stereo views of the electron density of the export complex structure .............58

Figure 3-21 Structure of the RanGTP•Xpo4•eIF5A export complex.................................59

Figure 3-22 Structure of Xpo4 in the export complex and HEAT repeat organization....... 61

Figure 3-23 Complexes at the asymmetric unit and Xpo4 contact sites...........................62 
Figure 3-24 Conformational differences between the Xpo4 molecules in the asymmetric

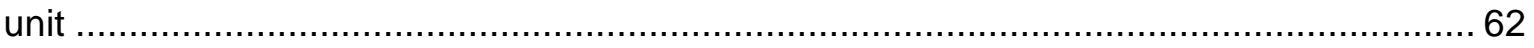

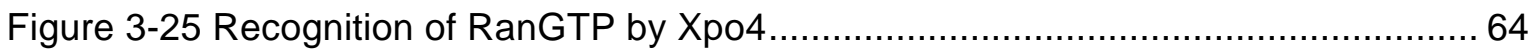

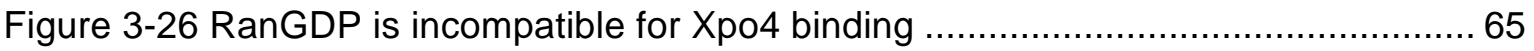

Figure 3-27 Structure of the export complex and domains of elF5A................................ 66

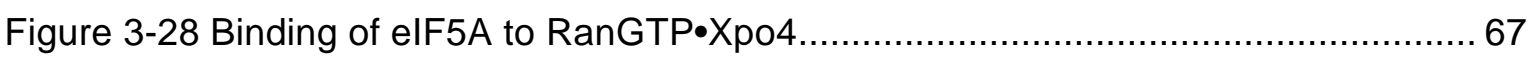

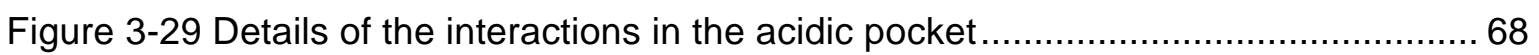

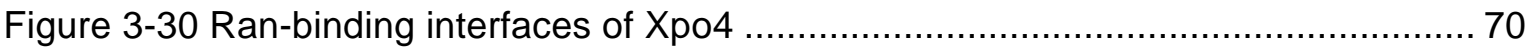

Figure 3-31 $\mathrm{N}$ terminal interaction interface of Xpo4 is essential for RanGTP binding ...... 71

Figure 3-32 Ran-binding interface 2 and 4 are required for proper elF5A binding ........... 72

Figure 3-33 $\mathrm{N}$ terminal docking of elF5A is crucial for Xpo4 binding ............................. 73

Figure 3-34 Hypusine-containing loop is essential for Xpo4 binding .............................. 74

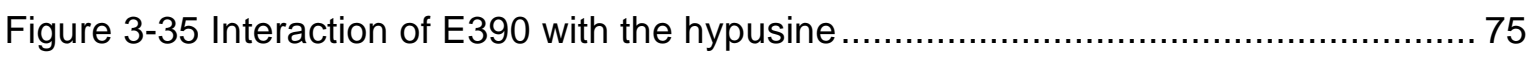

Figure 3-36 $\mathrm{N}$ terminal docking of elF5A is also essential for export activity of Xpo4 ...... 76

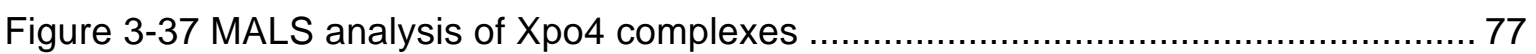

Figure 3-38 Identification of minimal Xpo4-binding region of Sox2 ................................ 78

Figure 3-39 Reconstitution of the import complex with Sox2 fragment............................. 79

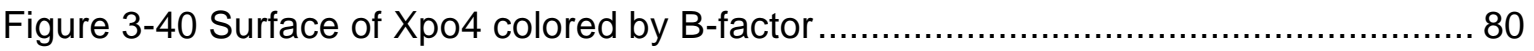

Figure 3-41 Reconstitution of the import complex with truncated Xpo4 and initial

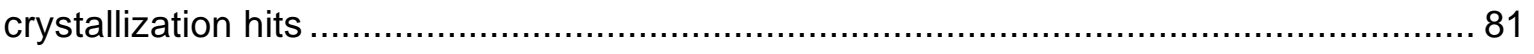

Figure 3-42 Reconstitution of RanGTP-Xpo4 complex .............................................. 83

Figure 3-43 Xpo7 affinity chromatography with cytoplasmic HeLa extract ........................ 86

Figure 3-44 Xpo7 affinity chromatography using cytoplasmic HeLa extracts prepared with SILAC method 88

Figure 3-45 MS analysis of forward and reverse experiments ..................................... 89

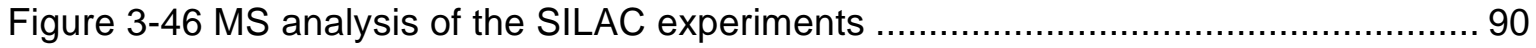

Figure 3-47 Identified candidate proteins enrich Xpo7 from a cytoplasmic extract in a

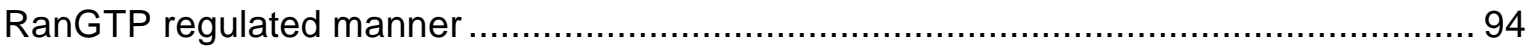

Figure 3-48 Binding assay with candidates and recombinant Xpo7 …........................... 95

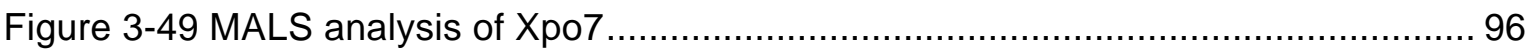

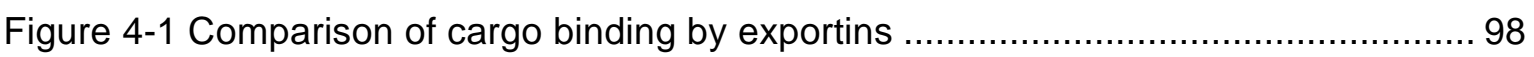

Figure 4-2 Cytoplasmic disassembly of the export complex ......................................... 101

Figure 4-3 Recognition of elF5A isoforms by Xpo4 ..................................................... 103

Figure 5-1 An example MRC plate of a grid screen design ........................................ 132 


\section{LIST OF TABLES}

Table 2-1 Functionally characterized vertebrate NTRs of the $\operatorname{Imp} \beta$ family and their

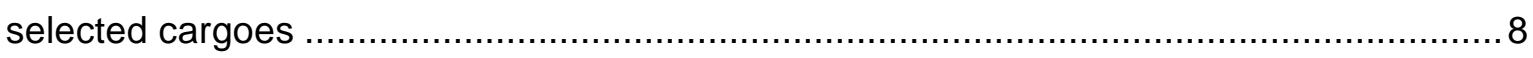

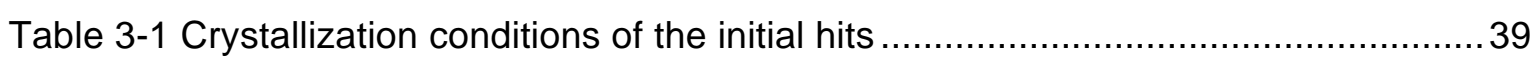

Table 3-2 Identified peptides that are used to determine the protease cleavage sites ..... 45

Table 3-3 Data collection and refinement statistics for RanGTP•Xpo4•elF5A complex ....57

Table 3-4 Mapping of Xpo4 boundaries for stable Sox2 binding .................................. 80

Table 3-5 Mapping of Xpo4 boundaries for ligand binding ............................................ 83

Table 3-6 The list of RanGTP dependent Xpo7 cargoes............................................... 91

Table 3-7 The list of potential Xpo7 import substrates ................................................... 92

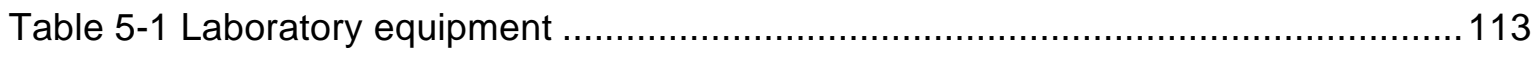

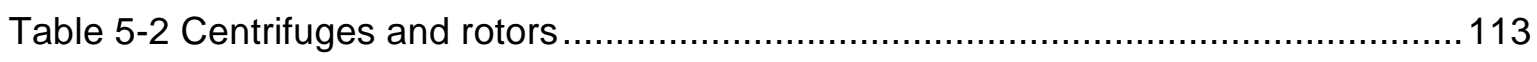

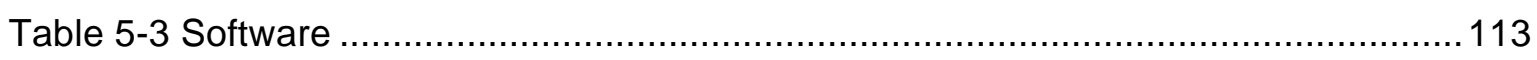

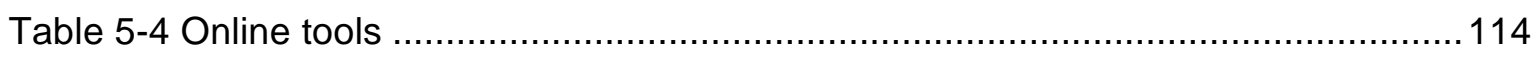

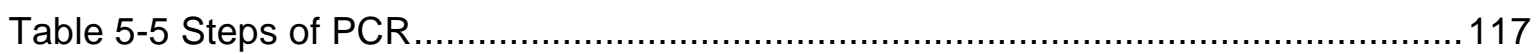

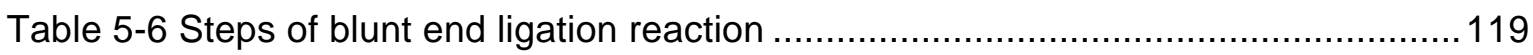

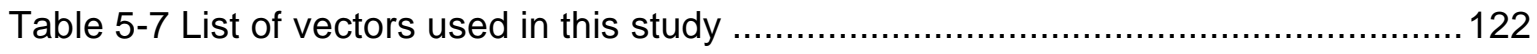

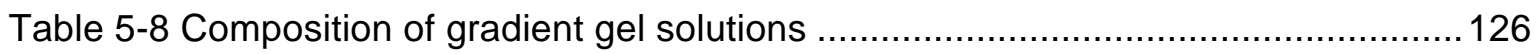

Table 7-1 Uniprot identifiers of the proteins that were significantly enriched in the

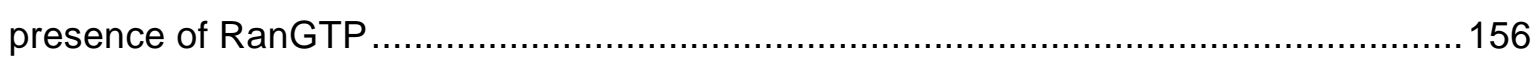

Table 7-2 Uniprot identifiers of the proteins that were significantly enriched in the absence of RanGTP. 


\section{LIST OF ABBREVIATIONS}

\begin{tabular}{|c|c|}
\hline A280 & Absorbance at $\lambda=280 \mathrm{~nm}$ \\
\hline ADP & Adenosine 5'-diphosphate \\
\hline ATP & Adenosine 5'-triphosphate \\
\hline BSA & Bovine serum albumin \\
\hline C-terminus & Carboxy-terminus \\
\hline CAS & Cellular apoptosis susceptibility (Exportin 2) \\
\hline cNLS & Classical nuclear localization signal \\
\hline CRM1 & Chromosomal region maintenance 1 (Exportin 1) \\
\hline DAP & 1,3-diaminopropane \\
\hline DHS & Deoxyhypusine synthase \\
\hline DLS & Dynamic light scattering \\
\hline DMSO & Dimethyl sulfoxide \\
\hline DNA & Deoxyribonucleic acid \\
\hline $\mathrm{DOHH}$ & Deoxyhypusine hydroxylase \\
\hline DTT & Dithiothreitol \\
\hline E.coli & Escherichia coli \\
\hline EDTA & Ethylenediaminetetraacetic acid \\
\hline elF & Eukaryotic translation initiation factor \\
\hline elF5A(Dhp) & Deoxyhypusine-containing elF5A \\
\hline elF5A(Hpu) & Hypsuine-containing elF5A \\
\hline elF5A(Lys) & Lysine-containing (non-modified) elF5A \\
\hline FG repeat & Phenylalanine-glycine repeat \\
\hline GAP & GTPase-activating protein \\
\hline GDP & Guanosine 5'-diphosphate \\
\hline GFP & Green fluorescent protein \\
\hline GTP & Guanosine 5'-triphosphate \\
\hline GTPase & GTP hydrolase \\
\hline His14 (or H14) & Amino terminal tag of 14 Histidine residues \\
\hline HEAT repeat & $\begin{array}{l}\text { Class of protein repeats (Huntingtin, Elongation factor } 3 \text {, Protein } \\
\text { phosphatase } 2 A \text {, TOR } 1 \text { ) }\end{array}$ \\
\hline HEPES & 4-(2-hydroxyethyl)-1-piperazineethanesulfonic acid \\
\hline HIV & Human immunodeficiency virus \\
\hline hnRNP & Heterogeneous nuclear ribonucleoprotein \\
\hline hs & Homo sapiens \\
\hline Hypusine & Hydroxypurescine-lysine; Nع-(4-amino-2-hydroxybutyl)lysine \\
\hline IBB & Importin $\beta$-binding domain of Importin $\alpha$ \\
\hline IMAC & Immobilized metal affinity chromatography \\
\hline $\operatorname{Imp}$ & Importin \\
\hline IPTG & Isopropyl- $\beta$-D-thiogalactopyranoside \\
\hline $\mathrm{kDa}$ & Kilodalton \\
\hline King cobra & Ophiophagus hannah \\
\hline LB & Luria-Bertani (lysogeny broth, medium) \\
\hline MALS & Multi angle light scattering \\
\hline MBP & Maltose binding protein \\
\hline MDa & Megadalton \\
\hline MES & 2-(N-morpholino)ethanesulfonic acid \\
\hline $\mathrm{mm}$ & Mus musculus \\
\hline MPI-BPC & Max Planck Institute for Biophysical Chemistry \\
\hline
\end{tabular}




\begin{tabular}{|c|c|}
\hline MW & Molecular weight \\
\hline N-terminus & Amino-terminus (start of a protein) \\
\hline NAD & Nicotinamide adenine dinucleotide \\
\hline ncNLS & Non-classical nuclear localization signal \\
\hline NE & Nuclear envelope \\
\hline NES & "Leucine-rich" nuclear export signal \\
\hline NLS & Nuclear localization signal \\
\hline NPC & Nuclear pore complex \\
\hline NTF2 & Nuclear transport factor 2 \\
\hline NTR & Nuclear transport receptor \\
\hline Nup & Nucleoporin \\
\hline$o / n$ & Overnight \\
\hline OAc & Acetate \\
\hline $\mathrm{OB}$ & Oligonucleotide-binding \\
\hline $\mathrm{OD}_{600}$ & Optical density $\lambda=600 \mathrm{~nm}$ \\
\hline OD600 & Optical densitiy of a sample measured at a wavelength of $600 \mathrm{~nm}$ \\
\hline PBS & Phosphate-buffered saline \\
\hline PDB & RCSB protein data bank \\
\hline PEG & Polyethylene glycol \\
\hline $\mathrm{Pi}$ & Inorganic phosphate \\
\hline PKI & Protein kinase $\mathrm{A}$ inhibitor \\
\hline PMSF & Phenylmethylsulfonyl fluoride \\
\hline r.m.s.d & Root mean square deviation \\
\hline Ran & Ras-related nuclear protein \\
\hline RanBP & Ran-binding protein \\
\hline RanGAP & RanGTPase-activating protein \\
\hline RanGDP & GDP-bound Ran \\
\hline RanGEF & Ran guanine nucleotide exchange factor \\
\hline RanGTP & GTP-bound Ran \\
\hline Ras & Rat sarcoma \\
\hline RCC1 & Regulator of chromosome condensation 1 (see also "RanGEF") \\
\hline RNA & Ribonucleic acid \\
\hline rpm & Rounds per minute \\
\hline sc & Saccharomyces cerevisiae \\
\hline SDS-PAGE & Sodium dodecyl sulfate-polyacrylamide gel electrophoresis \\
\hline SEC & Size exclusion chromatography \\
\hline SUMO & Small ubiquitin like modifier \\
\hline SV40 & Simian virus 40 \\
\hline TB & Terrific broth medium \\
\hline TEV & Tobacco etch virus \\
\hline Tris & 2-amino-2-hydroxymethyl-1,3-propanediol \\
\hline$w / v$ & Weight per volume \\
\hline$w / w$ & Weight per weight \\
\hline WT & Wild type \\
\hline Хро & Exportin \\
\hline YT & Yeast extract tryptone medium \\
\hline z & (in "zz") IgG-binding domain of the Staphylococcal protein A \\
\hline A280 & Absorbance at $\lambda=280 \mathrm{~nm}$ \\
\hline
\end{tabular}

Standard single-letter amino acid codes and the International System of units (SI) were used. 


\section{SUMMARY}

Nucleocytoplasmic exchange is essential for eukaryotic cells. It proceeds through nuclear pore complexes and is largely mediated by Importin $\beta$-like nuclear transport receptors (Imp $\beta$-like NTRs). According to the direction of the transport, Imp $\beta$-like NTRs are classified as importins or exportins. Exportin 4 (Xpo4) is a bidirectional receptor that can function both as importin and exportin. Xpo4 mediates export of the eukaryotic translation initiation factor 5A (elF5A) and Smad3 as well as import of transcription factors such as Sox2 and SRY. So far, it has been unclear how Xpo4 facilitates transport of structurally diverse cargoes to opposing compartments.

In order to understand the mechanisms of Xpo4-dependent bidirectional transport, in my PhD work, I aimed at crystallizing the cargo-bound and unbound complexes of Xpo4. Here I present the crystal structure of the RanGTP.Xpo4•eIF5A export complex. The structure shows that Xpo4 recognizes not just a linear peptide, but the two folded domains of elF5A at the same time. elF5A contains hypusine, a unique amino acid with two positive charges, that is essential for cell viability and for elF5A function in translation. The hypusine docks into a deep, acidic pocket of Xpo4. The interactions at the acidic pocket are essential for export complex formation and elF5A export by Xpo4. Therefore, hypusine is a critical element of elF5A's complex export signature. The structure also reveals that Ran promotes elF5A binding through conformational changes in Xpo4, including the stabilization of a conserved acidic loop. Similar acidic loops in unidirectional importins have critical function in cargo assembly and disassembly processes. Therefore, this detail also gives mechanistic hints about how Xpo4 can act as a bidirectional transport receptor.

In a parallel project, I aspired to identify novel transport substrates for exportin 7 (Xpo7). To address this, I employed an Xpo7 affinity chromatography to enrich Xpo7 interaction partners from a cytoplasmic extract. This revealed not only further Xpo7 export substrates but also several potential import cargoes. The results suggest that in addition to its characterized role in nuclear export, Xpo7 could function as a nuclear import receptor. Therefore, similar to Xpo4, Xpo7 can be another bidirectional nuclear transport receptor. 


\section{INTRODUCTION}

\subsection{Nucleus}

The compartmentalization of the eukaryotic cell into membrane-bound organelles is the main difference between eukaryotes and prokaryotes. The most prominent organelle and the defining feature of the eukaryotes, as their name implies (eu, "true" and karyo, "kernel"), is the cell nucleus. The nucleus is enclosed by the nuclear envelope $(\mathrm{NE})$, a double membrane structure that separates the genome from the cytoplasm.

The nucleocytoplasmic compartmentation offers numerous advantages to the eukaryotes. Firstly, the confinement of the genome into the nucleus protects it from mechanical and metabolic damages and therefore contributes genomic stability, which allows eukaryotes to handle considerably larger genomes (Görlich and Kutay, 1999). Secondly, the nucleus separates nuclear processes like DNA replication, transcription and mRNA splicing from the translation in the cytoplasm. It hereby provides spatiotemporal regulation of gene expression. Moreover, the spatial separation of transcription from translation allows eukaryotes to easily handle intron-containing genes to exploit alternative mRNA splicing. As a result, eukaryotes increased the coding potential of their genomes in respect to a given number of genes. Finally, the possibility to control the localization of specific molecules such as transcription factors adds another layer of regulation.

Regardless of the numerous benefits, the separation of the nucleus and the cytoplasm necessitates a nucleocytoplasmic exchange of materials. Since translation takes place only in the cytoplasm, all proteins that are needed in the nucleus, such as the components of DNA repair and replication machinery have to be imported from the cytoplasm. Conversely, translation depends on nuclear products such as the mRNAs, tRNAs and the ribosomal subunits which need to be exported to the cytoplasm. In fact, more than a million macromolecules per second are actively transported between the nucleus and the cytoplasm (Ribbeck and Görlich, 2001). 


\subsection{Nucleocytoplasmic Transport}

Nuclear pore complexes (NPCs) conduct nucleocytoplasmic transport. NPCs are proteinaceous channels that are embedded in the NE and formed at the sites where the inner and outer nuclear membranes meet (Watson, 1954). With molecular weights of $\sim 66 \mathrm{MDa}$ in yeast (Rout and Blobel, 1993) and $125 \mathrm{MDa}$ in vertebrates (Reichelt et al., 1990), NPCs constitute one of the largest complexes in the cell. Despite such gigantic sizes however, NPCs are assembled only by $\sim 30$ different proteins, called nucleoporins (Nups). Individual Nups can occur in different copy numbers per NPC (Ori et al., 2013). Given the eight-fold symmetry of NPCs (Gall, 1967), these copy numbers are usually assumed to be multiples of eight. The NPC scaffold encloses a large aqueous channel (see e.g. Eibauer et al., 2015), which is guarded by a permeability barrier formed by cohesive phenylalanine-glycine (FG) domains (Frey and Görlich, 2007; Patel et al., 2007; Hulsmann et al., 2012)

The permeability barrier allows efficient passive diffusion of small molecules and proteins up to $5 \mathrm{~nm}$ in diameter (or 20-40 $\mathrm{kDa}$ in mass) whereas becomes limiting as the size exceeds the passive diffusion limit (Bonner, 1975; Mohr et al., 2009). In contrast, larger macromolecules depend on nuclear transport receptors (NTRs) for facilitated transport (reviewed in Görlich and Kutay, 1999).

\subsubsection{Importin $\beta$-like nuclear transport receptors}

Most of the facilitated transport is mediated by the NTRs of the Importin $\beta(\operatorname{Imp} \beta)$ family (occasionally also referred to as $\beta$-karyopherins), which have the ability to interact with the FG repeats of the NPC and overcome the size limit of the permeability barrier. NTRs constantly shuttle between the cytoplasm and the nucleus, bind to their cargoes on one side of the NE, pass through the NPC as complexes and release them on the other side. Despite their poor sequence homology (8-15\% identity), Impß-like NTRs show similar domain organization (see below). In addition to their large size (90-130 kDa), these receptors are characterized by their acidic isoelectric point ( $\mathrm{pl}$ 4.6-6.0), their affinity for phenylsepharose, and their ability to bind Ran (Görlich and Kutay, 1999; Ribbeck and Görlich, 2002). 
Ran (Ras-related nuclear protein) is a member of the small ras-like GTPases (Bischoff and Ponstingl, 1991a). It was the first identified nuclear transport factor (Melchior et al., 1993; Moore and Blobel, 1993). Its function in nucleocytoplasmic transport is best explained by the RanGTP gradient model (Görlich et al., 1996b; Izaurralde et al., 1997). GDP-bound Ran (inactive state, from now on referred to as RanGDP) is found predominantly in the cytoplasm, while the GTP-bound Ran (active state, referred to as RanGTP) is present exclusively in the nucleus. The differential localization of Ran species is maintained by the asymmetric distribution of Ran effector proteins. The guanine nucleotide exchange factor RCC1 (regulator of chromosome condensation 1), which facilitates GDP to GTP exchange on Ran (Bischoff and Ponstingl, 1991b; Klebe et al., 1995) is bound to chromatin (Ohtsubo et al., 1989), therefore RanGTP is produced only in the nucleus. On the other hand, the Ran GTPase activating protein 1 (RanGAP1) is exclusively cytoplasmic (Hopper et al., 1990; Matunis et al., 1996; Mahajan et al., 1997). RanGAP1 stimulates the intrinsic GTPase activity of Ran, decreasing the RanGTP levels in the cytoplasm (Bischoff et al., 1994; Becker et al., 1995; Klebe et al., 1995). Notably, when RanGTP is bound to NTRs, RanGAP1 alone cannot act on Ran, instead it requires the assistance of a special class of Ran-binding proteins, RanBP1 (Coutavas et al., 1993) or RanBP2/Nup358 (Yokoyama et al., 1995), to activate the RanGTPase in NTR complexes (Bischoff and Görlich, 1997; Kutay et al., 1997).

Impß-like NTRs bind RanGTP at least 1000-fold stronger than RanGDP (Görlich et al., 1996b). RanGTP binding acts like a switch, altering the cargo binding behavior of the NTR. Therefore, the RanGTP gradient across the NE drives the directionality of the transport (Görlich et al., 1996b; Izaurralde et al., 1997). According to the direction they carry their cargoes, Imp $\beta$-like NTRs are classified as exportins and importins. Figure 2-1 illustrates their transport cycles. Exportins bind their cargoes in the nucleus, where the RanGTP level is high. Export complexes traverse NPCs as trimeric RanGTP-exportin-cargo complexes and are dissociated upon hydrolysis of Ran-bound GTP in the cytoplasm. Free exportin goes back to the nucleus for another round of export. Importins, on the other hand, function in the opposite manner. Importins bind their cargoes in the cytoplasm, where the RanGTP level is low, and traverse the NPCs as dimeric import 
complexes. In the nucleus, RanGTP binding to the importin dissociates the import complex, releasing the import cargo into the nucleus. The newly formed importinRanGTP complex travels back to the cytoplasm, where the RanGTP effectors disassemble it from the importin, allowing the importin to perform another import cycle. Although these transport processes use the metabolic energy supplied by RanGTP, the translocation across the NPC per se is energy-independent (Kose et al., 1997; Schwoebel et al., 1998; Nachury and Weis, 1999; Ribbeck et al., 1999).

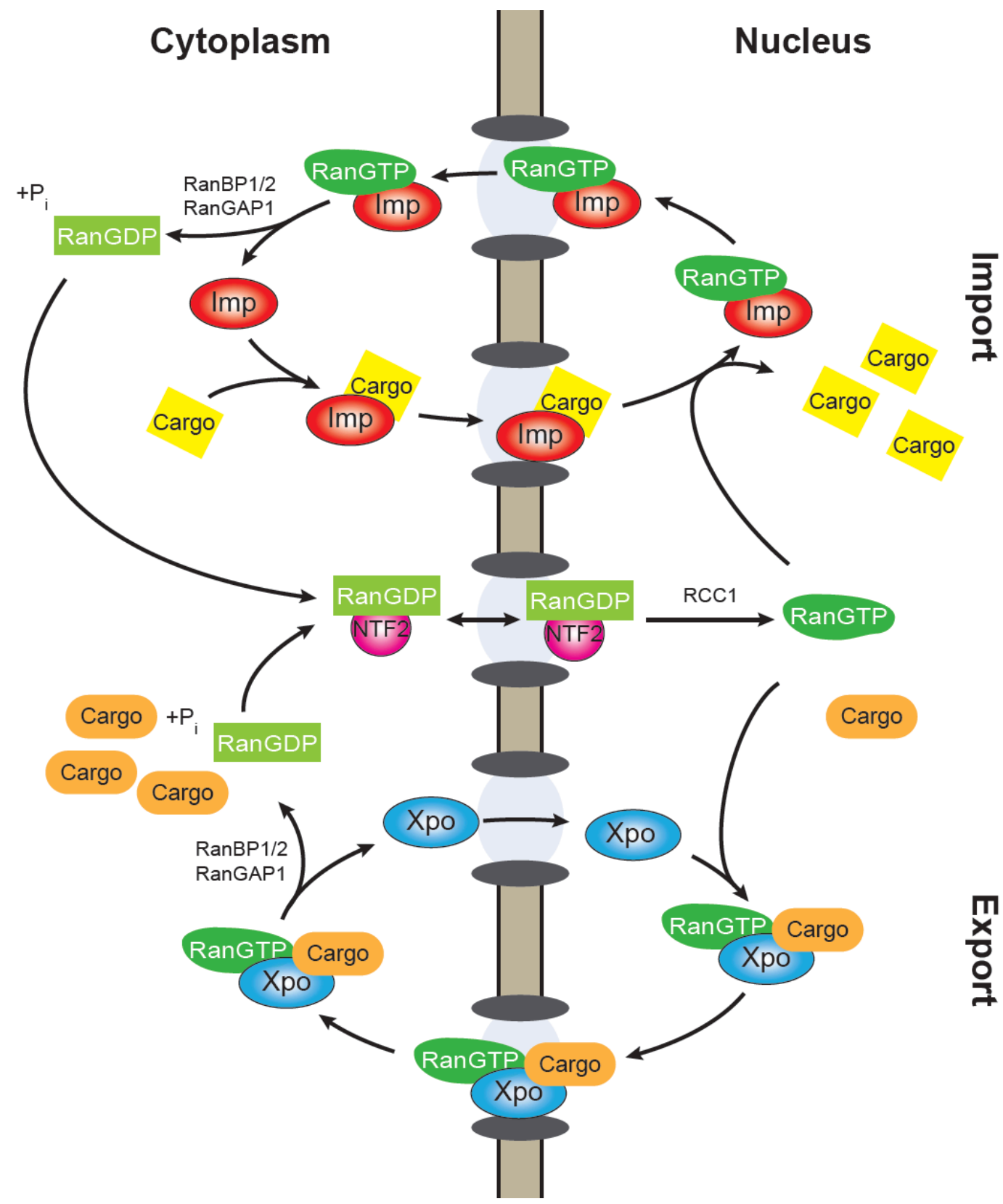

Figure 2-1 Overview of the nucleocytoplasmic transport cycles

Importins and exportins are abbreviated as Imp and Xpo, respectively. See text for the details. Adapted from Görlich \& Kutay, 1999. 
Each round of import and export removes one RanGTP molecule from the nucleus. Another small NTR, nuclear transport factor 2 (NTF2), counteracts the depletion of Ran by shuttling RanGDP back to the nucleus (Figure 2-1; (Ribbeck et al., 1998). Based on its structure (Bullock et al., 1996) and sequence (Moore and Blobel, 1994), NTF2 is not a member of Imp $\beta$ family. NTF2 is a $15 \mathrm{kDa}$ protein and found in the cell as homodimer, which transports two RanGDP molecules. Dissociation of RanGDP from NTF2 occurs after the conversion of GDP to GTP by RCC1 (Ribbeck et al., 1998).

The Imp $\beta$ family is the largest NTR class and comprises 21 members in vertebrates and 14 members in Saccharomyces cerevisiae. While most of these receptors transport cargoes only in one direction, some can mediate both import and export. The functionally characterized vertebrate NTRs and a selection of their respective cargoes are summarized in Table 2-1.

\begin{tabular}{|c|c|c|c|}
\hline NTR & Adapter & Cargoes & Selected references \\
\hline \multicolumn{4}{|l|}{ Importins } \\
\hline \multirow[t]{6}{*}{$\operatorname{Importin} \beta(\operatorname{Imp} \beta)$} & & Ribosomal proteins & (Jakel and Görlich, 1998) \\
\hline & & HIV Rev, HIV Tat & (Truant and Cullen, 1999) \\
\hline & & Histones & $\begin{array}{l}\text { (Mosammaparast et al., } \\
\text { 2001; Muhlhausser et al., } \\
\text { 2001) }\end{array}$ \\
\hline & Importin $\alpha$ & Classical NLS-cargoes & (Görlich et al., 1995) \\
\hline & Snurportin1 & $\mathrm{m}_{3} \mathrm{G}$-capped U-snRNPs & (Huber et al., 1998) \\
\hline & Importin 7 & Histone H1 & (Jakel et al., 1999) \\
\hline \multirow{5}{*}{$\begin{array}{l}\text { Transportin } 1+2 \text { (Trn, } \\
\text { Imp } \beta-2)\end{array}$} & & hnRNP proteins & (Pollard et al., 1996) \\
\hline & & Ribosomal proteins & (Jakel and Görlich, 1998) \\
\hline & & TAP/NXF1 & (Truant and Cullen, 1999) \\
\hline & & Histones & (Muhlhausser et al., 2001) \\
\hline & & c-FOS & (Arnold et al., 2006) \\
\hline $\begin{array}{l}\text { Transportin SR 1+2 } \\
\text { (TrnSR, Trn3) }\end{array}$ & & SR proteins & (Kataoka et al., 1999) \\
\hline \multirow[t]{2}{*}{ Importin 4} & & Ribosomal proteins & (Jakel et al., 2002) \\
\hline & & Histones & $\begin{array}{l}\text { (Mosammaparast et al., } \\
\text { 2001; Muhlhausser et al., } \\
\text { 2001) }\end{array}$ \\
\hline Importin 5 & & $\begin{array}{l}\text { Ribosomal proteins } \\
\text { Histones }\end{array}$ & (Jakel and Görlich, 1998) \\
\hline \multirow[t]{2}{*}{ Importin 7} & & Ribosomal proteins & (Jakel and Görlich, 1998) \\
\hline & & ERK2, SMAD3, MEK1 & (Chuderland et al., 2008) \\
\hline
\end{tabular}




\begin{tabular}{|c|c|c|c|}
\hline NTR & Adapter & Cargoes & Selected references \\
\hline \multirow[t]{2}{*}{ Importin 8} & & Argonuate proteins & (Weinmann et al., 2009) \\
\hline & & SRP19 & (Dean et al., 2001) \\
\hline \multirow[t]{2}{*}{ Importin 9} & & Ribosomal proteins & (Jakel et al., 2002) \\
\hline & & Histones & $\begin{array}{l}\text { (Mosammaparast et al., } \\
\text { 2001; Muhlhausser et al., } \\
\text { 2001) }\end{array}$ \\
\hline \multirow[t]{2}{*}{ Importin 11} & & UbcM2 & (Plafker and Macara, 2000) \\
\hline & & rpL12 & (Plafker and Macara, 2002) \\
\hline \multicolumn{4}{|l|}{ Exportins } \\
\hline \multirow[t]{5}{*}{ CRM1 (Exportin 1) } & & Leu-rich NES cargoes & $\begin{array}{l}\text { (Fischer et al., 1995; Wen } \\
\text { et al., 1995; Fornerod et } \\
\text { al., 1997) }\end{array}$ \\
\hline & HIV Rev & HIV genomic RNA & (Fischer et al., 1995) \\
\hline & & Snurportin1 & (Paraskeva et al., 1999) \\
\hline & NMD3 & $\begin{array}{l}\text { 60S pre-ribosomal } \\
\text { subunit }\end{array}$ & $\begin{array}{l}\text { (Ho et al., 2000; Thomas } \\
\text { and Kutay, 2003) }\end{array}$ \\
\hline & PHAX/CBC & $\mathrm{m}^{7} \mathrm{G}$-capped UsnRNAs & $\begin{array}{l}\text { (Izaurralde et al., 1995; } \\
\text { Ohno et al., 2000) }\end{array}$ \\
\hline CAS (Exportin 2) & & Importin as & (Kutay et al., 1997) \\
\hline Exportin-t (Xpot) & & tRNAs & $\begin{array}{l}\text { (Arts et al.., 1998; Kutay et } \\
\text { al., 1998) }\end{array}$ \\
\hline \multirow[t]{4}{*}{ Exportin 5} & & tRNAs, eEF1A & $\begin{array}{l}\text { (Bohnsack et al., 2002; } \\
\text { Calado et al., 2002) }\end{array}$ \\
\hline & & dsRNAs & $\begin{array}{l}\text { (Brownawell and Macara, } \\
\text { 2002) }\end{array}$ \\
\hline & & Pre-miRNAs & $\begin{array}{l}\text { (Yi et al., 2003; Bohnsack } \\
\text { et al., 2004; Lund et al., } \\
2004 \text { ) }\end{array}$ \\
\hline & & $\begin{array}{l}\text { 60S pre-ribosomal } \\
\text { subunit }\end{array}$ & (Wild et al., 2010) \\
\hline Exportin 6 & & Actin-profiling complex & (Stuven et al., 2003) \\
\hline Exportin 7 & & p50RhoGAP, 14-3-3o & (Mingot et al., 2004) \\
\hline \multicolumn{4}{|l|}{ Bidirectional NTRs } \\
\hline \multirow[t]{2}{*}{ Importin 13} & & $\begin{array}{l}\text { UBC9, MagoY14 } \\
\text { (import) }\end{array}$ & (Mingot et al., 2001) \\
\hline & & elF1A (export) & (Mingot et al., 2001) \\
\hline \multirow[t]{3}{*}{ Exportin 4} & & elF5A (export) & (Lipowsky et al., 2000) \\
\hline & & SMAD3 (export) & (Kurisaki et al., 2006) \\
\hline & & Sox2, SRY (import) & (Gontan et al., 2009) \\
\hline
\end{tabular}

Table 2-1 Functionally characterized vertebrate NTRs of the Imp $\beta$ family and their selected cargoes 


\subsubsection{Imp $\beta$-like nuclear export receptors}

To date, eight $\operatorname{Imp} \beta$ family exportins have been identified in vertebrates (Table 2-1), however, only four of them (CAS, Exportin-t, Exportin 5 and CRM1) have functional orthologues in other eukaryotes. CAS (Exportin 2) is a specialized exportin and exports only one type of protein: Importin a (Impa; Kutay et al., 1997). Impa, the import adaptor of Imp $\beta$, recognizes nuclear localization signals (NLSs, see below) and confers nuclear import of NLS-containing proteins. After each import cycle, Impa is recycled back to the cytoplasm by CAS for another import cycle. Similarly, Exportin-t (Xpot) is a dedicated exportin and mediates the nuclear export of tRNAs that are properly processed and modified (Kutay et al., 1998; Lipowsky et al., 1999). Exportin 5 (Xpo5) also exports tRNAs (Bohnsack et al., 2002). Moreover, Xpo5 transports additional RNAs, such as doubled stranded RNAs (dsRNAs) and precursor microRNAs (pre-miRNAs), from the nucleus to the cytoplasm (Brownawell and Macara, 2002; Bohnsack et al., 2004; Lund et al., 2004). Finally, in vertebrates, Xpo5 functions in the export of 605 pre-ribosomal subunits (Wild et al., 2010). Similarly, CRM1 (Xpo1) also supplies the cytoplasm with the RNA-based cargoes, including UsnRNAs, signal recognition particles (SRPs) as well as the 40S and 60S ribosomal subunits (Ciufo and Brown, 2000; Ohno et al., 2000; Thomas and Kutay, 2003). Moreover, unlike the abovementioned exportins, CRM1 exports a wide variety of cargoes that are structurally unrelated and thus it is involved in many cellular processes (reviewed in Hutten and Kehlenbach, 2007; Güttler and Görlich, 2011; Ishizawa et al., 2015 and see below).

In summary, Xpot, Xpo5 and CRM1 deliver the nuclear products to the cytoplasm, while CAS recycles Impa and contributes to the Imp $\beta$-dependent nuclear import. Therefore, all of these conserved exportins fulfill functions that are necessitated by the compartmentalization of the eukaryotic cells. Furthermore, exportins perform an additional fundamental function: they preserve the identity of the nucleus by constantly exporting the cytoplasmic factors from the nucleus, to confine their localization and therefore their activity to the cytoplasm. As described before, the NPCs are not absolute barriers and allow free diffusion of proteins smaller than the passive diffusion limit (20-40 kDa). In fact, even far larger objects can diffuse through the NPCs when the sufficient time is provided. Consequently, proteins 
whose functions have to be restricted to the cytoplasm, e.g. the components of the translation machinery or whose access to the nucleus needs to be dependent on a stimulus, e.g. transcription factors can enter the nucleus and give rise to undesirable outcomes. In order to counteract the leakage of such proteins, exportins continuously depletes them from the nucleus. CRM1 and Xpo5, for example, export most of the essential translation factors from the nucleus to limit their activity to the cytoplasm and therefore play an essential role in separating the transcription from the translation (Bohnsack et al., 2002). By recognizing hundreds of cytoplasmic proteins, CRM1 is the major actor of this task. However, higher eukaryotes employ additional exportins, Exportin 4, Exportin 6, Exportin 7, and Importin 13, to deplete additional cytoplasmic factors from the nucleus.

So far, only one export cargo has been identified for Exportin 6 (Xpo6) and Importin 13 (Imp13), which transport actin and elF1A, respectively, to the cytoplasm (Mingot et al., 2001; Stuven et al., 2003). Exportin 4 (Xpo4) and Exportin 7 (Xpo7) will be the focus of this dissertation and therefore will be presented in the following sections.

\section{Exportin 4 (Xpo4)}

Xpo4 was initially identified as an export receptor of elF5A (eukaryotic translation initiation factor $5 \mathrm{~A}$ ), and later was shown to export Smad3 as well (Lipowsky et al., 2000; Kurisaki et al., 2006). Moreover, Xpo4 mediates the nuclear import of Soxtype transcription factors, i.e. Sox2 and SRY (Gontan et al., 2009). In other words, Xpo4 transports distinct cargoes into opposite compartments and is the third characterized bidirectional NTR after Imp13 (Mingot et al., 2001) and Msn5p, the Xpo5 orthologue in S. cerevisiae (Yoshida and Blobel, 2001). In addition, Xpo4 acts as a co-regulator of Sox9, another Sox-type transcription factor, by suppressing the Sox9-mediated transcription without affecting its cellular localization (Tsuchiya et al., 2011). Xpo4 has recently been characterized as a tumor suppressor protein in murine model of hepatocellular carcinoma (HCC) (Zender et al., 2008).

Xpo4 orthologues can be found in all vertebrates and arthropods as well as in some plants and even in slime mold Dictyostelium discoideum but not in Drosophila melanogaster and in fungi. The closest relative of Xpo4 within the $\operatorname{Imp} \beta$ 
superfamily is Xpo7, which together form a separate subgroup in the phylogenetic tree (Kutay et al., 2000).

\section{Exportin 7 (Xpo7)}

Xpo7 was isolated from HeLa cells via affinity chromatography on immobilized RanGTP and referred as RanBP16 (Ran-binding protein 16; Kutay et al., 2000). Subsequently, RanBP16 was shown to export p50RhoGAP and 14-3-3o from human and $X$. laevis nuclei and therefore characterized as the last member of the Impß-like exportins (Mingot et al., 2004). The same study revealed the translation initiation factor elF4AI and the subunits of the endosomal retromer (Vps35, Vps26 and Vps29) as additional Xpo7 binders. Predominant cytoplasmic localization of these proteins and their RanGTP-dependent interaction with Xpo7 suggested that these proteins could also be export substrates of Xpo7 (Mingot et al., 2004). Additionally, Xpo7 mediates export of the serine/threonine kinase LKB1 via the adapter STRADa (Dorfman and Macara, 2008). Identification of several structurally distinct proteins as export substrates and the lack of a common signal sequence suggested that $\mathrm{Xpo7}$, similar to CRM1, might be a broad-spectrum exportin (Mingot et al., 2004; Dorfman and Macara, 2008).

Xpo7 is conserved in vertebrates and also exists in several other higher eukaryotes including $D$. melanogaster, $C$. elegans and even in some plants such as cotton and rice (Kutay et al., 2000). Xpo7 is ubiquitously expressed in all human tissues (Kim et al., 2014). The erythroid-specific isoform of Xpo7, Xpo7B, is highly expressed at the onset of the terminal erythroid differentiation and is required for the erythroid nuclear condensation (Hattangadi et al., 2014). Moreover, in these cells, the cytoplasmic migration of the nuclear proteins prior to enucleation is inhibited upon Xpo7 knockdown, which suggests that Xpo7 is necessary for the nuclear export of these proteins.

\subsubsection{Nuclear transport signals}

Nucleocytoplasmic transport of cargoes is a highly controlled process and requires specific interaction between the cargo and the NTR. Whether a cargo is recognized and transported by an NTR is determined by the presence of nuclear transport signals on the cargoes. Whereas some of these transport signals are 
quite complex and involve three-dimensional features of the cargo, some other transport signals are as simple as a few amino acid-short peptides that the fusion of these signals direct any protein to the corresponding compartment. Such transport signatures are termed as nuclear import and nuclear export signals.

The very first examples of the nuclear localization signals (NLSs) were identified in the large T-antigen of simian virus 40 (SV40), which consisted a short patch of basic amino acids (PKKKRKVE; Kalderon et al., 1984) and in Xenopus laevis nucleoplasmin, where the basic patch was separated by a short spacer (Robbins et al., 1991). The SV40 type (monopartite) and the nucleoplasmin type (bipartite) signals are referred as the classical nuclear localization signals (cNLSs) and form the major NLS class of the Impß-dependent nuclear import. However, cNLSs are not recognized directly by Imp $\beta$. Instead, Impa binds to cNLS (Görlich et al., 1994), which, in turn, is recognized by $\operatorname{Imp} \beta$ via its Imp $\beta$-binding (IBB) domain (Görlich et al., 1996a). Nevertheless, not all Imp $\beta$ import cargoes require Impa. The proteins that contain non-classical NLSs (ncNLSs) interact directly with $\operatorname{Imp} \beta$. A 29 amino acid peptide from the parathyroid hormone-related protein (PTHrP; Lam et al., 1999) and the highly basic (pl 12.2) beta-like import receptor-binding (BIB) domain of rpL23a (Jakel and Görlich, 1998) are the well-known examples of ncNLSs.

As described before, CRM1 is the most versatile exportin and transports hundreds of proteins to the cytoplasm. The simplest nuclear export signals (NESs) are the so-called leucine-rich export signals of the CRM1-dependent export pathway. These NESs were initially identified as the short peptides comprising four interspersed hydrophobic residues. The first examples of this kind were characterized in the protein kinase A inhibitor (PKI) and HIV Rev (Fischer et al., 1995; Wen et al., 1995). Later, the PKI type (LALKLAGLDI) and the Rev type (LPPLERLTL) NESs have been identified in several other CRM1 cargoes, and these two have formed the major NES classes. A comprehensive study by Güttler and his colleagues (2010) redefined the NES consensus to be $x-\Phi-x_{2}-\Phi-x_{3}-\Phi-x_{2-3}-\Phi-x-\Phi$ (where $\Phi$ is hydrophobic and $\mathbf{x}$ is any amino acid) for PKI type and $\Phi-\Phi-x-\Phi-x-\Phi-x-\Phi$ for Rev type. 


\subsection{The structural features of Imp $\beta$-like NTRs}

All NTRs of the Imp $\beta$ family share a common architecture and are composed of the so-called HEAT repeats (Görlich et al., 1997; Chook and Blobel, 1999; Cingolani et al., 1999; Vetter et al., 1999a), named after the four proteins of this class: huntingtin, elongation factor 3 , the PR65/A subunit of protein phosphatase 2A (PP2A) and the lipid kinase TOR (Andrade and Bork, 1995). HEAT repeats are $\sim 40$ amino acid motifs, which made up of two antiparallel $\alpha$-helices (called $A$ and B) that are connected by a short linker. In Imp $\beta$-like NTRs, about 20 of these repeats pack side by side, generally with a clockwise rotation between the successive repeats. This gives rise to a uniform right-handed superhelical structure with the $A$ helices forming the outer convex surface and the B helices forming the inner concave surface (Figure 2-2a). This repetitive organization confers flexibility to the Imp $\beta$-like NTRs, which leads to diverse shapes from a closed ring to an open supercoil (Figure 2-2b). In addition, the flexibility of the structure contributes to cargo binding and cargo release (Conti et al., 2006).

The helices of the HEAT repeats contain hydrophobic amino acids that make intra- and inter-repeat contacts and form a continuous hydrophobic core. These continuous blocks are sealed by the first and last helices. Moreover, the successive $A$ helices form hydrophobic pockets necessary for the interaction with the FG repeats of the NPC components (Bayliss et al., 2002). In contrast, the B helices usually establish the interaction interfaces with Ran and the cargo (see below). 


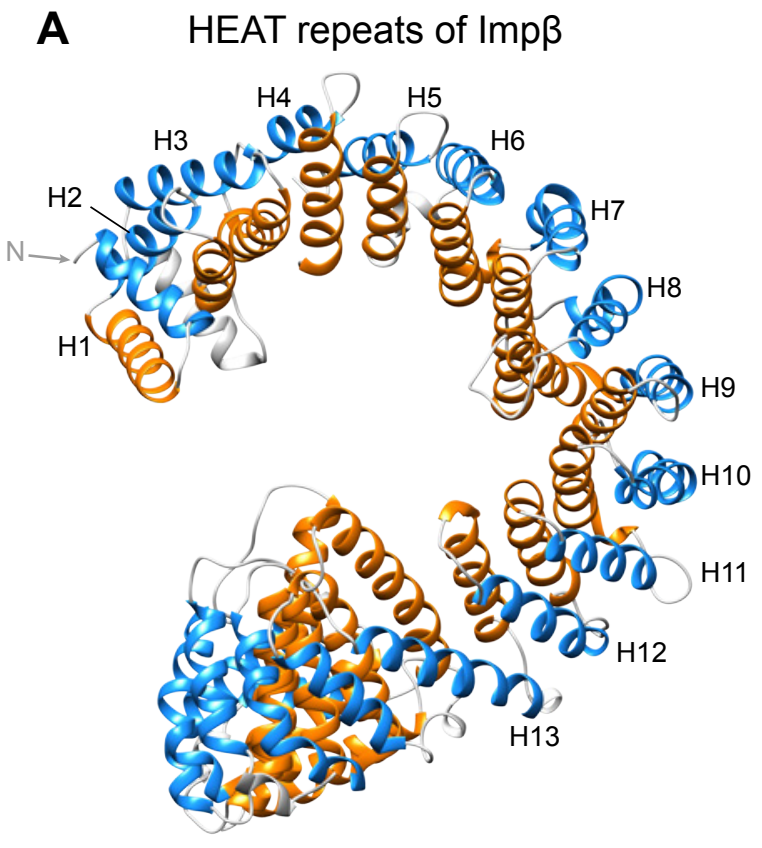

B Flexibility of Impß-like NTRs
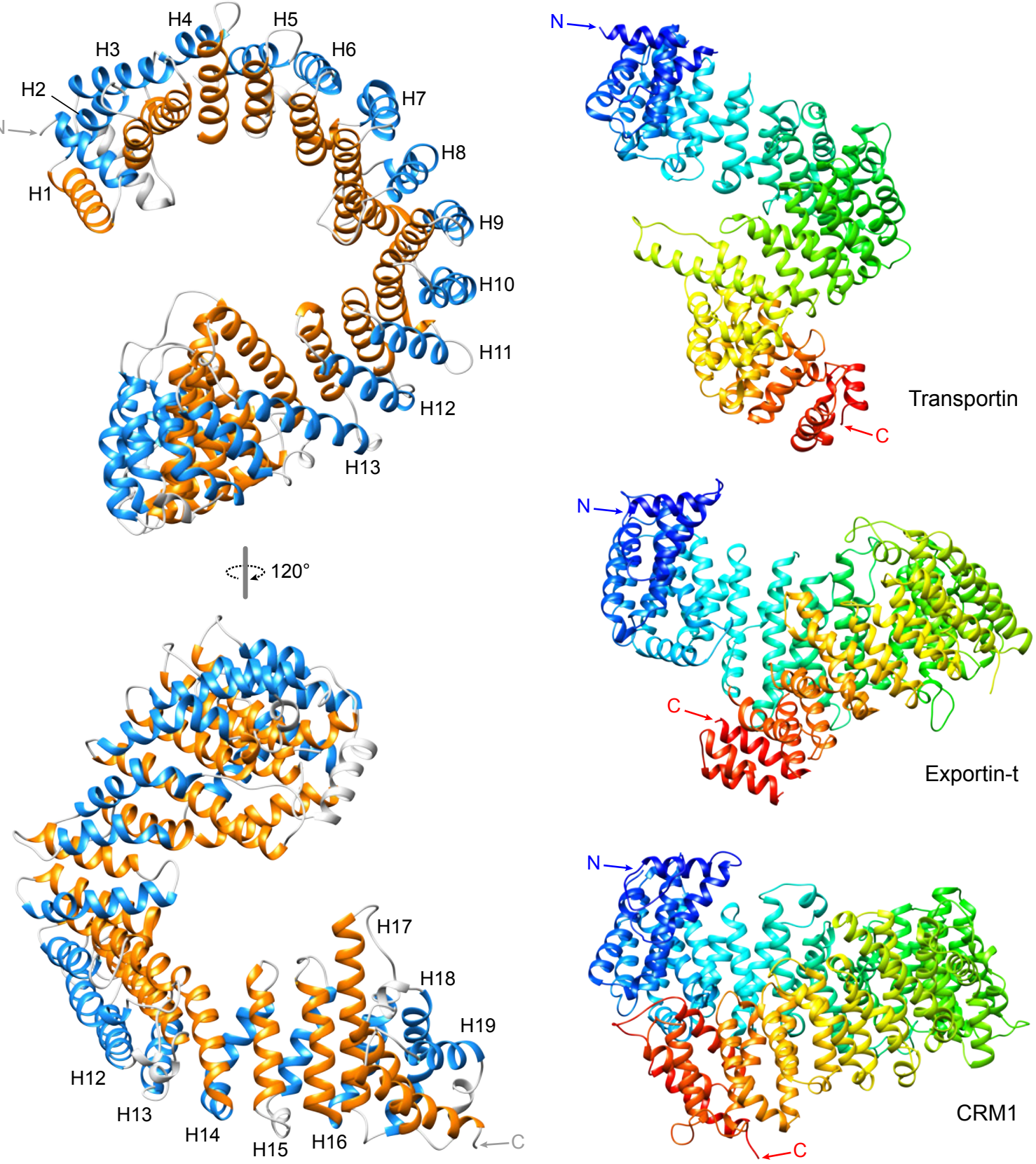

Figure 2-2 Architecture of Imp $\beta$-like NTRs

A) HEAT repeat structure of $\operatorname{Imp} \beta$ (from the Imp $\beta$ RanGTP structure, PDB ID 2BKU, Lee et al., 2005) is shown in two different orientations. HEAT repeats are numbered according to Lee et al., 2005. A and B helices are highlighted in blue and orange, respectively, while the loops and non-HEAT helices are colored in grey. N- and C-termini of the molecule are indicated. See text for further details B) The structures of Transportin (2OT8, Cansizoglu and Chook, 2007), Exportin-t (3ICQ, Cook et al., 2009) and CRM1 (3GJX, Monecke et al., 2009) are depicted to illustrate the conformational flexibility of NTRs. The transport receptors are shown with a color gradient from blue ( $\mathrm{N}$-terminus) to red (C-terminus). 


\subsubsection{The regulator of NTR•cargo interaction: Ran}

As described above, RanGTP drives the directionality of the transport by mediating either cargo release (for importins) or cargo binding (for exportins) and therefore plays a central role for the nucleocytoplasmic transport. For this reason, in this section, I will briefly introduce the structures of the nucleotide-bound Ran.

To date, several structures of Ran alone or in complex with Ran-binding proteins have been solved (Vetter and Wittinghofer, 2001). Figure 2-3 shows representative structures of Ran bound to GDP and GTP (Scheffzek et al., 1995; Vetter et al., 1999b; Partridge and Schwartz, 2009). The structure of Ran resembles the so-called the $G$ domain of the Ras-like small GTPases, which is composed of six $\beta$-strands surrounded by five $\alpha$-helices (Scheffzek et al., 1995). Nucleotide recognition is mediated by the loops of the $\mathrm{G}$ domain, which involves several hydrogen bonds as well as a $\mathrm{Mg}^{2+}$ ion. In addition to the $\mathrm{G}$ domain, Ran has a characteristic C-terminal extension (Scheffzek et al., 1995).

Comparison of GDP- and GTP-bound Ran structures reveals conformational differences at three regions, referred as "switch regions" (Figure 2-3; Scheffzek et al., 1995; Vetter et al., 1999b). RanGDP adopts more compact folding, where the C-terminal helix (Switch III) fold onto the "back" of the Ran. In addition, the acidic DEDDDL motif at the very extreme C-terminus (residues 211-216), which is not resolved in the crystal structure, probably contacts the so-called "basic patch" (Vetter et al., 1999b). Upon nucleotide exchange, the switch regions reorganize markedly. Switch I adopts almost a new shape and packs against the GTP, now making extensive contacts with it. In addition, switch I clashes with the loop of switch III, forcing it to reorganize. Now, switch III has an extended conformation and does not contact the $\mathrm{G}$ domain, and thus the basic patch of the Ran becomes free. The change in switch II is minor but functionally important. Switch II contains the $\operatorname{Gln}^{69}$ residue, which is crucial for GTPase activity (Bischoff et al., 1994). In the GTP state, Gln ${ }^{69}$ is brought to close proximity to the $Y$-phosphate of GTP. Moreover, the position and the coordination of the $\mathrm{Mg}^{2+}$ ion change as well. 



\section{RanGTP}

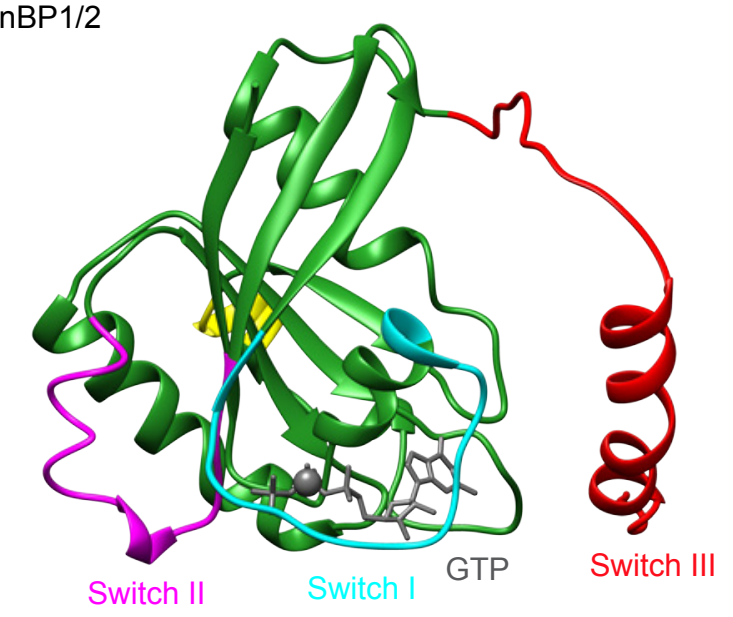

$\% 180^{\circ}$
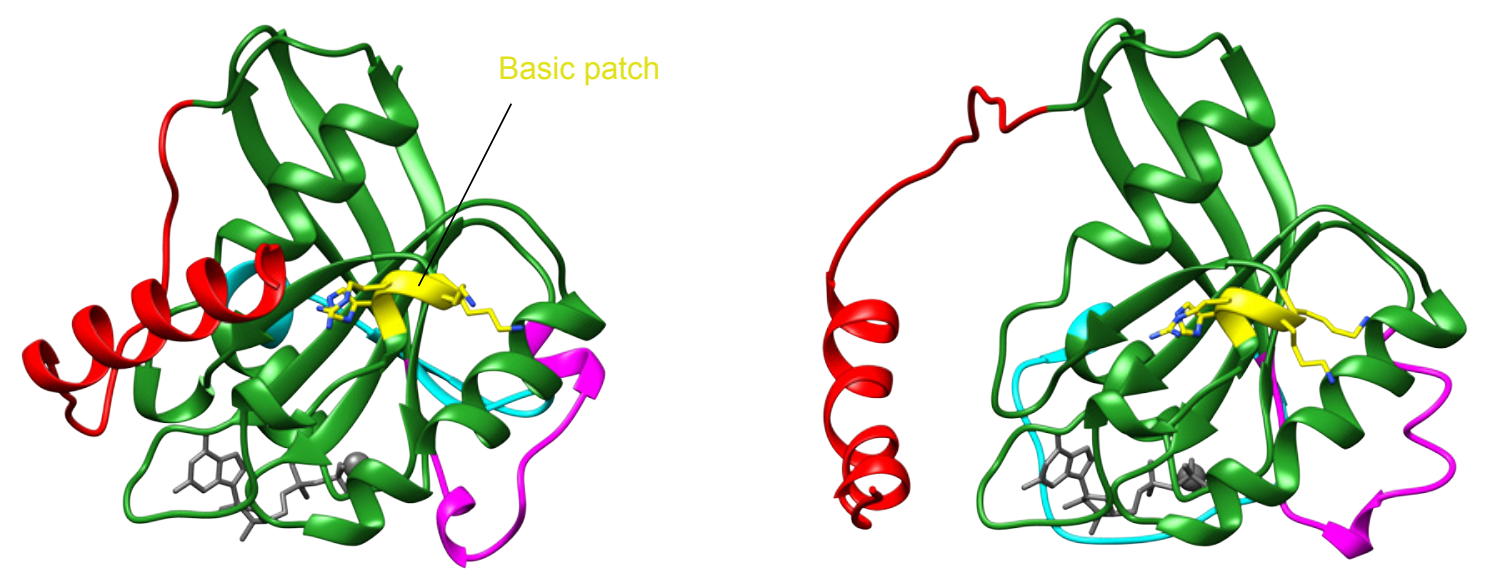

Figure 2-3 Comparison of GDP- and GTP-bound structures of Ran

The structures of Ran bound to GDP (left) and GTP (right) are shown in ribbon representations. $\mathrm{Mg}^{2+}$ ions and the nucleotides are shown as spheres and sticks, respectively. The core of the protein ( $G$ domain) is colored in green, while the parts that undergo drastic conformational changes are colored in cyan (switch I, residues 30-47), magenta (switch II, residues 65-80), red (switch II, residues 177-216) and yellow (basic patch, residues 139-142) and indicated accordingly. In the lower panel, models were rotated $180^{\circ}$ to view the "back" of Ran. Residues of the basic patch were shown in yellow sticks, while nitrogens in blue. DEDDDL motif (residues 211-216) at the very end of the molecule is not resolved in the crystal structures, however, it likely folds onto the basic patch of Ran. RanGDP and RanGTP were taken from the structures with PDB ID 3GJO (Partridge and Schwartz, 2009) and 1RRP (Vetter et al., 1999b), respectively.

\subsubsection{Interaction of RanGTP with Imp $\beta$-like NTRs}

Impß-like NTRs make use of the RanGTP gradient across the NPCs by discriminating RanGTP from RanGDP. However, they do not directly contact the bound GTP, instead they perceive the regions that are different between the GDPand GTP-bound states, the switch I and II. In addition, NTRs interact with Ran at its basic patch and at the invariant loops, which contact the bound nucleotide. 
The structures of RanGTP bound to $\operatorname{Imp} \beta$ and Transportin had showed how importins recognize the GTP state of Ran (Chook and Blobel, 1999; Vetter et al., 1999a). N-terminal HEAT repeats, which are the most conserved among the NTRs; (Görlich et al., 1997), form the first interaction interface and contact switch II as well as the back of Ran (Figure 2-4). Second interaction interface is formed by HEAT repeats (HEAT 7 and 8 ) at the middle portion of the importins. The so-called acidic loop at HEAT 8 interacts with, among others, the basic patch. In RanGDP, this region is shielded by the switch III, and therefore would not be accessible by importins. Finally, B helices of the C-terminal repeats (HEAT 12-15) establish the third interaction interface, which contacts the loops of Ran that hold guanine base. While, Imp $\beta$ also interacts with switch I of Ran via its C-terminal repeats, no such interaction has been described for Transportin so far. It should be noted that the switch III of Ran does not contribute to RanGTP binding. Indeed, it is disordered in the NTR structures solved to date.

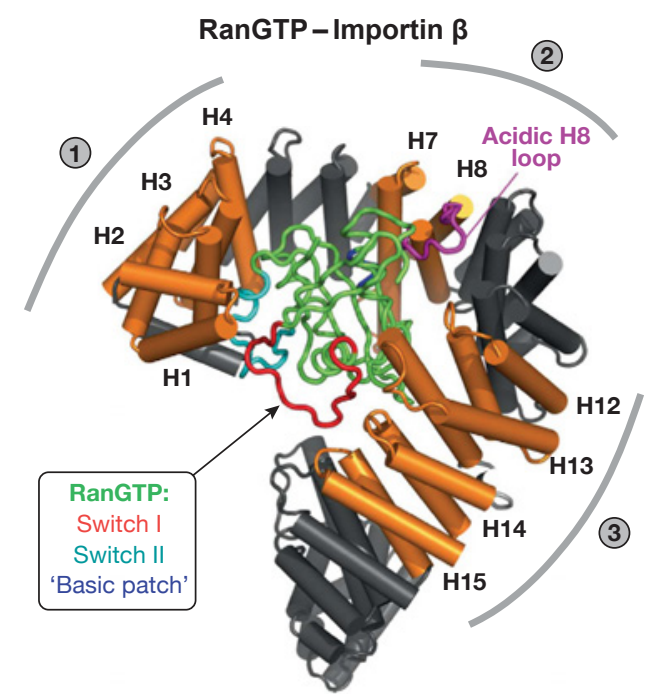

Figure 2-4 Interaction of RanGTP with Imp $\beta$

The figure (adapted from Güttler and Görlich, 2011) shows the recognition of Ran by Imp $\beta$. Ran is shown in a tube representation and colored in green. Important parts of Ran are colored and indicated accordingly. The helices of $\operatorname{Imp} \beta$ are depicted as cylinders and colored in black. Those HEAT repeats that are involved in RanGTP recognition are numbered and highlighted in orange. Ran-binding regions are labeled as encircled numbers. 

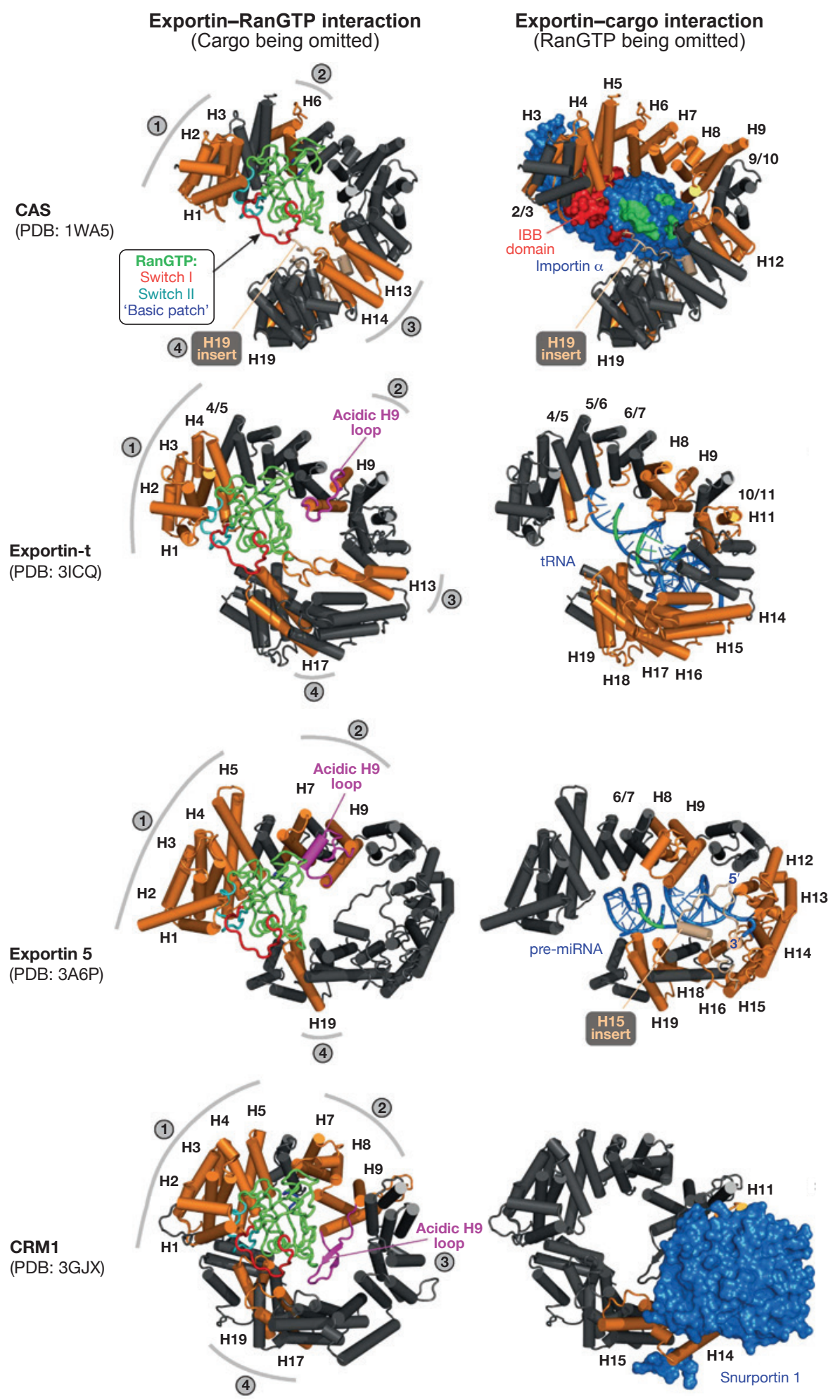

Figure 2-5 Interaction of exportins with RanGTP and respective cargoes

Export complex structures of CAS, Exportin-t, Exportin 5 and CRM1 are shown in two different views. Left: Exportin-RanGTP interactions are illustrated as described in Figure 2-4. Structures were aligned according to Ran. Right: Similar view as in left. To show the exportin-cargo interactions, RanGTP is removed and surface or ribbon representation of respective cargoes are shown in blue, while the regions contact Ran are colored in green. The figure was adapted from Güttler and Görlich, 2011. 
So far, the structures of several exportins (CRM1, CAS, Xpot, Xpo5 and Imp13) in cargo-bound form have been elucidated. Although the general principles of RanGTP recognition by exportins appear somehow similar to the importins, specific aspects are different and exclusive to the exportins (Matsuura and Stewart, 2004; Cook et al., 2009; Monecke et al., 2009; Okada et al., 2009; Grunwald et al., 2013). All exportins contain an additional C-terminal Ran-binding interface (HEAT 17-19), which (except Xpo5) always contacts switch I of Ran (Figure 2-5, region four). In the case of Xpo5, switch I is recognized by the $\mathrm{N}$-terminal repeats. Consequently, Ran is wrapped by the exportins from two sides. Exportins also differ from importins in terms of the acidic loop and the interaction with the basic patch of Ran. With the exception of CRM1, exportins do not contact the basic patch. Indeed, CAS does not even posses an acidic loop (Figure 2-5).

\subsubsection{Cargo recognition by Imp $\beta$-like nuclear export receptors}

Unlike importins, which either bind to the cargo or Ran, exportins couple Ran binding to cargo loading. In other words, exportins accommodate the cargo and Ran at the same time. The recruitment of the cargo and Ran occurs in a cooperative manner where the binding of one increases the affinity of exportin towards the other one. Structures of the exportins in the cargo-bound (nuclear) form as well as in the cargo-free (cytoplasmic) form revealed that such cooperativity is achieved by direct interactions of Ran with the cargo or by the conformational changes throughout the exportin (reviewed in Güttler and Görlich, 2011).

As mentioned before, CAS recycles the import adaptor Impa back to the cytoplasm. In the export complex, both Ran and Impa are hold by the $\mathrm{N}$ - and C-terminal HEAT repeats and accommodated on the inner surface of CAS such that a negatively charged C-terminal part of Impa binds the basic patch of Ran (Figure 2-5, Matsuura and Stewart, 2004).

Xpot and Xpo5 are the two RNA-specific exportins of the cell. While Xpot specifically exports mature tRNAs, Xpo5 mediates export of additional RNAs such as pre-miRNA and dsRNAs. With respect to the cargo-binding mode, Xpot and Xpo5 export complex structures resemble that of CAS (Figure 2-5, Cook et al., 
2009; Okada et al., 2009). Both exportins wrap the cargo and Ran. In addition, similar to CAS, the basic patch of Ran engages interactions with negatively charged portions of the cargoes. Both exportins recognizes different RNAs in a sequence-independent manner. They accomplish this by probing the phosphoribose backbone of the RNAs. Although the overall charges of Xpot and Xpo5 are negative, positively charged residues on the inner surface mediate the interaction with the cargo in both cases. In spite of all the similarities, the shapes of these exportins differ in the export complexes: Xpot assumes relatively circular arrangement whereas Xpo5 forms a U-shaped structure. This difference might explain how Xpo5 can recognize diverse RNAs (Güttler and Görlich, 2011).

With respect to the shape of the receptor and the recruitment of the cargo, CRM1 export complexes differ from the exportins described above. While CAS, Xpot and Xpo5 utilize mostly the B helices to hold the cargo at the inner surface of the exportin, this mechanism does not exist in CRM1; instead the cargo is recruited to the outer surface (Figure 2-5, Monecke et al., 2009). In the export complex, CRM1 forms a toroid-like structure, where $\mathrm{C}$-terminal helices touch the inter-repeat loops of the N-terminal repeats (see also Figure 2-2). Similar to the other $\operatorname{Imp} \beta$-like receptors, Ran is positioned on the inner surface of CRM1. Indeed, CRM1 almost completely encircles Ran: $\mathrm{N}$ - and C-terminal HEAT repeats of Ran interact with switch II and I, respectively; HEAT 7 and 8 shield the basic patch of Ran; the acidic loop in HEAT 9 interacts with the loops holding the nucleotide base and locks Ran to the $\mathrm{N}$ - and $\mathrm{C}$-terminal repeats (Figure 2-5). Such a recognition topology leaves no space for the cargo binding. Therefore, the export cargo binds to outside of the toroid, far away from the Ran-binding region, which represents a special case where Ran and the cargo do not interact. This kind of cargo recognition mode does not enforce any size limitation onto the cargoes, which allows CRM1 to carry cargoes as large as ribosomal subunits (Güttler and Görlich, 2011).

Structures of CRM1 export complexes with different NESs revealed how CRM1 exports a large number of structurally diverse cargoes (Dong et al., 2009; Monecke et al., 2009; Güttler et al., 2010). The A helices of the HEATs 11 and 12 form a hydrophobic cleft, which the hydrophobic $\Phi$ residues of the NES dock into. NESs that differ in their $\Phi$ spacing docks into the same pocket by adopting 
different backbone conformations. While, for instance, Snurportin1 NES is mostly a-helical in the hydrophobic cleft, HIV Rev NES adopts an almost linear conformation. As a result, these studies showed that any protein could be a CRM1 cargo as long as it has an accessible peptide, which can adopt certain conformation to place its $\Phi$ residues into the hydrophobic cleft.

It would be an elegant mechanism for the eukaryotic cell to append an NES to all proteins that must be exclusively cytoplasmic. Although, in the cell, most of the proteins are exported in this way, several other proteins require the assistance of different exportins. Presence of four additional exportins in the higher eukaryotes might even indicate a different aspect of the export mechanism, which would not be provided by the CRM1-dependent export pathway. How the bidirectional transporter Xpo4 mediates export of elF5A will be the main focus of this study.

\section{4 elF5A}

The eukaryotic translation initiation factor $5 \mathrm{~A}$ (elF5A) is a small (17 kDa), abundant, highly conserved and essential protein found in all eukaryotes and archaea (Gordon et al., 1987; Park et al., 1993; Chen and Liu, 1997). Its bacterial ortholog, elongation factor $\mathrm{P}$ (EF-P), also exists in all bacteria; therefore, elF5A/EF-P is a universally conserved protein (Kyrpides and Woese, 1998; Saini et al., 2009).

elF5A is the only protein known to contain the unusual amino acid hypusine [NE-(4-amino-2-hydroxybutyl)lysine] (Park et al., 1993). Hypusine (hydroxyputrescine-lysine) was first found in bovine brain tissue by Shiba et al. (1971) and later identified in all animal tissues both in free form and in protein (Imaoka and Nakajima, 1973) yet brain having the highest amount of free hypusine (Nakajima et al., 1971). In addition to hypusine, brain contains hypusine derivatives like $\gamma$-aminobutyrylhypusine and $\beta$-alanylhypusine that might act as neurotransmitter (Park et al., 1993). To date, no biosynthetic pathway has been identified for the formation of free hypusine; therefore, degradation of elF5A has been suggested as the source of free hypusine (Park et al., 1993). Finally, observation of hypusine in the excreted urine by Nakajima et al. (1971) led to the hypothesis that the hypusine might be the end product of lysine metabolism. 
Hypusine is indispensible for elF5A function (Schnier et al., 1991) as well as for cell viability and cell proliferation. It is produced post-translationally in two consecutive reactions called hypusination (Figure 2-6). First, deoxyhypusine synthase (DHS) transfers the 4-aminobutyl group from spermidine (using NAD as cofactor) to a specific lysine residue ( $\mathrm{K} 50$ in human, $\mathrm{K} 51$ in yeast) of elF5A to yield deoxyhypusine. Later, second carbon of the aminobutyl moiety is hydroxylated by deoxyhypusine hydroxylase $(\mathrm{DOHH})$ to form hypusine. Inhibition of either deoxyhypusine synthesis by spermidine analogs or deoxyhypusine hydroxylation by chelators prevents growth in mammalian cells (Hanauske-Abel et al., 1994; Lee et al., 1995). Moreover, elF5A-K51R mutant that prevents hypusination does not replace wild type eIF5A, indicating the essential function of hypusine in the cell (Schnier et al., 1991). Interestingly, although both DHS and DOHH are essential in mammalian cells, only DHS is required for cell viability in yeast (Park, 2006).

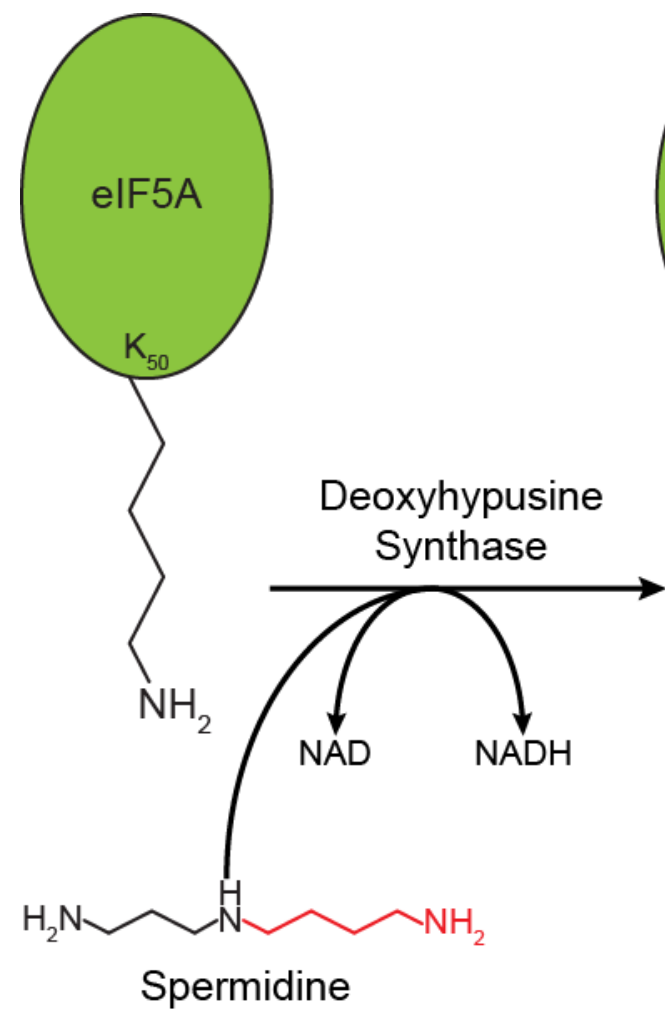

Deoxyhypusine
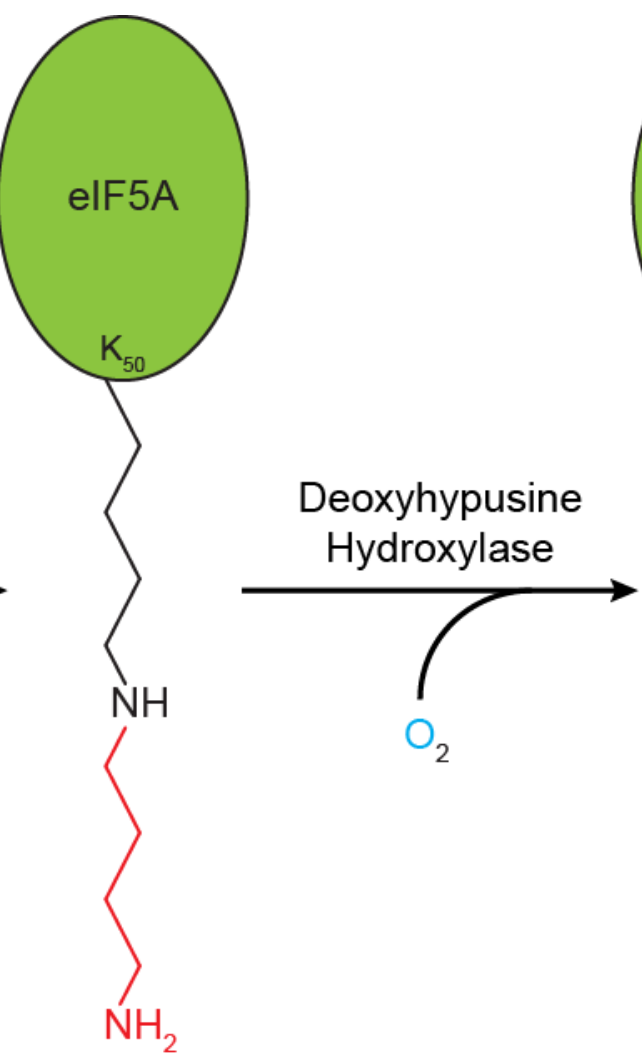

Hypusine

Figure 2-6 Posttranslational modification of elF5A

On the contrary, EF-P lacks hypusine. Nonetheless, another unique posttranslational modification, referred to as lysinylation, occurs on a specific 
lysine (K34) residue of EF-P (Yanagisawa et al., 2010; Peil et al., 2012). However, unlike elF5A, the lysine is not conserved in all bacteria, instead an arginine is found in most of EF-P, which was recently shown to be modified by a reaction called rhamnosylation (Lassak et al., 2015).

\begin{tabular}{|c|c|}
\hline И. jannaschii & TKQVNVGSLKVGQY \\
\hline H. butylicus & --VTYATLGELKVGSYIV \\
\hline mexicana & SDED- \\
\hline thaliana & PQQAGT IRKNGY \\
\hline . cerevisiae & MSDEE----HTFETADAG--SSATYPMQCSALRKNGFVT \\
\hline deum & MSDNEALDVEDYAQAGSG--ASLTFP IQCSALRKNGFVVI \\
\hline ster & MAELD----DQFETTDSG--ASTTYPMQCSALRKNGFVML \\
\hline G. $g$ & MAD-D----LDFETGDAG--ASATFPMQCSALRKNGFVVL \\
\hline H. $s$ & ETGDAG--ASATFPMQCSALRKNGFVV \\
\hline chii & I IDRRKGQVLAIMGI \\
\hline S & I IDKRIGQVIADMGD \\
\hline na & IVATDIFTGNRLEDQAPSTHNVE \\
\hline A. th & FVAIDIFTSKKLEDIVPS \\
\hline lae & LVAIDIFTGKKLEDLSP \\
\hline D. $d$ & ITAIDIFTGKKYEEICPSTHNID \\
\hline D. $n$ & MVGIDIF SNKKYEDICP STHNMD \\
\hline G. $\mathrm{g}$ & LVGIDIFTGKKYEDICPSTHNMD \\
\hline H. s & LVGIDIETGKKYEDICPSTHNMDVPNIKRNDFQLIGIQDG \\
\hline M. ja & -EGIEG-LEPGGE--VEYIEAVGQYKITRVIGGK---- \\
\hline H. b & -EDLKSKLQPGVE--VEYWVVMGRYMITRVRGAPKS-- \\
\hline L. $\mathrm{m}$ & -SHLSLMDDEGE-------SRE---------------- \\
\hline A. $t$ & LQQIKSGFDDGKDLVVSVMSAMGEEQINALKDIGPK-- \\
\hline b. & GDSLQTAFDEGKDLMVT I I SAMGEEAA I SFKEAARTD- \\
\hline D. $c$ & GKEITQMLKEGKEPLVSVISALGKEGVVSVKVSNN--- \\
\hline gaster & GEQLRLDFDSGKDLLCTVLKACGEECVIAIKTNTALDK \\
\hline G. $g$ & GREIEQKYDCGEEIITIHGARFTTS------------ \\
\hline H. sapiens & GKEIEQKYDCGEEILITVLSAMTEEAAVAIKAMAK--- \\
\hline
\end{tabular}

Figure 2-7 Multiple sequence alignment of elF5A from different species

elF5A sequences from archaeal (Methanocaldococcus jannaschii, Hyperthermus butylicus) and eukaryotic (Dictyostelium discoideum, Leishmania Mexicana, Arabidopsis thaliana, Saccharomyces cerevisiae, Drosophila melanogaster, Gallus gallus and Homo sapiens) organisms. The arrow marks the lysine residue that is converted to hypusine. The amino acid residues identical throughout archaea and eukaryotes are highlighted in red, completely conserved in eukaryotes are highlighted in orange and highly conserved in eukaryotes are highlighted in green.

elF5A is a highly conserved protein. Multiple sequence alignment of elF5A amino acid sequences from different species reveals high degree of conservation (Figure 2-7, Figure 2-8c). Remarkably, the amino acid sequence of the region surrounding the lysine that undergoes hypusination is identical in all eukaryotes, which might suggest the importance of this region for recognition by the enzymes or for the function of elF5A (Park et al., 1993). The sequence analysis also shows that the conservation is higher in the $\mathrm{N}$-terminus and the sequence similarity decreases towards the C-terminus. In addition, $\mathrm{N}$-terminus is shorter in archaea than in 
eukaryotes. Moreover, elF5A from human, slime mold and alfalfa can substitute yeast elF5A (Magdolen et al., 1994) indicating the functional conservation.

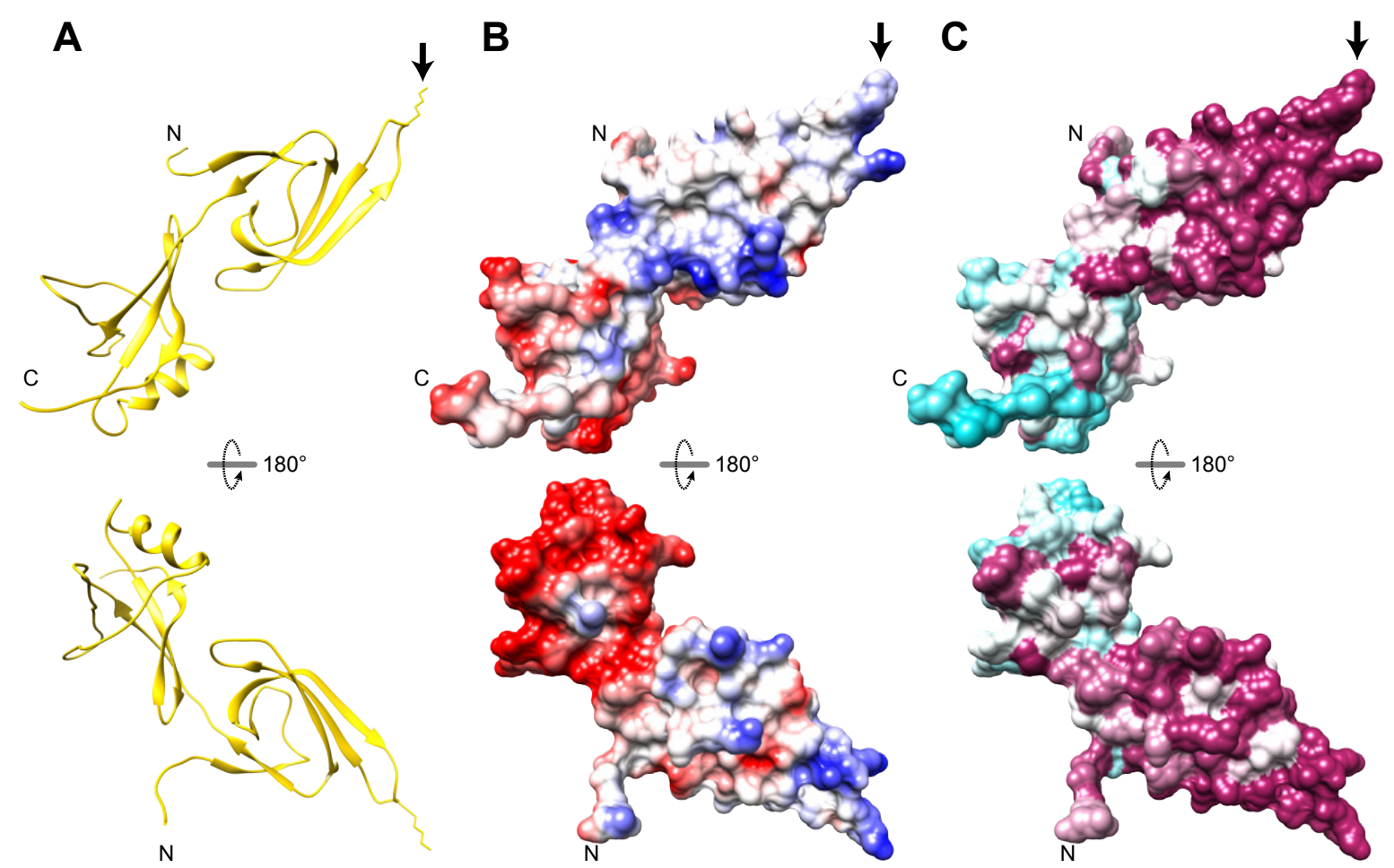

Figure 2-8 Structure of elF5A

A) 3D structure of yeast elF5A (PDB ID 3ER0) as ribbon representation with two orientations. $\mathrm{N}$ and $\mathrm{C}$ represent $\mathrm{N}$-terminus and $\mathrm{C}$-terminus, respectively. Lysine that is converted to hypusine is marked with arrow and shown as stick. B, C) elF5A is depicted as surfaces in the same orientation as in $(\mathbf{A})$. The surfaces are colored according to electrostatic potential (B) with a color gradient from red (negatively charged) to blue (positively charged) and according to conservation (C) with a gradient from cyan (variable) to maroon (conserved). Images were generated using UCSF Chimera.

Although elF5A gene is encoded by one gene in archaea, many eukaryotic organisms have two or more elF5A genes (Schnier et al., 1991; Jenkins et al., 2001; Wang et al., 2001; Thompson et al., 2004). These genes are differentially transcribed and the products of these genes are thought to play different roles in the cell. The human elF5A is encoded by EIF5A1 and EIF5A2 genes. EIF5A1 is constitutively expressed in all tissues, while EIF5A2 is expressed in testis, brain and highly expressed in certain cancer tissues and tumor cell lines (Park et al., 2010). Likewise, in yeast, two genes (TIF51A and TIF51B) encode elF5A proteins that are $90 \%$ identical and their expression is regulated according to the presence of oxygen as TIF51A is transcribed in aerobic conditions (Schnier et al., 1991). Nonetheless, both of these proteins can replace each other without having any effect on the growth rate and the protein content suggesting a functional similarity 
of these proteins. Interestingly, some plants contain more than two elF5A genes; Arabidopsis thaliana has three and tomato (Lycopersicon esculentum) has four elF5A isoforms that are expressed in different tissues (Thompson et al., 2004).

So far, the crystal structures of elF5A orthologues from several species including archaea, yeast, human and plants have been elucidated (Figure 2-8, Kim et al., 1998; Peat et al., 1998; Yao, 2003; Teng et al., 2009; Tong et al., 2009). They show that elF5A is composed of two globular domains connected by a flexible hinge region. $\mathrm{N}$-terminal domain is formed by six $\beta$-strands and resembles the $\mathrm{SH} 3$-like domains of other proteins related to translation. In addition, $\mathrm{N}$-terminal domain contains the hypusine in an extended, flexible, and highly conserved loop (Figure 2-8). Moreover, most of the basic residues are localized to the $\mathrm{N}$-terminal domain (Figure 2-7 and Figure 2-8), which gives rise to relatively positively charged region. In contrast, the C-terminal domain is almost entirely acidic and consists of an oligonucleotide-binding (OB)-fold found in nucleic acid-binding proteins. In fact, elF5A was shown to bind certain RNAs (Xu and Chen, 2001). Same study also revealed that in addition to the C-terminal domain, the hypusine is essential for sequence-specific interactions with RNAs.

\subsubsection{Identification of elF5A and its role in translation}

elF5A (formerly IF-M2Ba or elF4D) was initially purified from rabbit reticulocytes as a component of translation initiation system and was shown to stimulate translation in the model methionyl-puromycin synthesis assay (Kemper et al., 1976). Later, Benne and Hershey (1978) showed that unlike other initiation factors, elF5A was not necessary for the formation of 80 S ribosomal complexes and had no effect on globin synthesis. Similarly, elF5A did not stimulate poly(U)-dependent phenylalanine synthesis in the assay with the purified $80 \mathrm{~S}$ initiation complexes and elongation factors EF-1 and EF-2, eliminating the role of elF5A as an elongation factor (Benne and Hershey, 1978). Therefore, elF5A was suggested to stimulate the first peptide bond between the methionine and puromycin. However, subsequent studies in yeast revealed that the loss of elF5A had slight impact on the global protein synthesis (Kang and Hershey, 1994; Zuk and Jacobson, 1998). Based on this and the observation that the depletion of elF5A led to the accumulation of cells in G1 phase, it was suggested that elF5A may function in 
translation of certain proteins like those involved in $\mathrm{G} 1$ to $\mathrm{S}$ phase transition (Hanauske-Abel et al., 1994; Kang and Hershey, 1994).

Although the exact role of elF5A in translation had remained as a mystery for decades, recent studies demonstrated a specific role in translation elongation instead of initiation. In 2006, elF5A was shown to bind the translating $80 S$ ribosomes in a hypusine-dependent manner (Jao and Chen, 2006; Zanelli et al., 2006), restoring its possible function in translation. Afterwards, two independent groups illustrated elF5As function in translation elongation (Gregio et al., 2009; Saini et al., 2009). Later, EF-P was shown to be essential for translation of polyproline-containing proteins (Doerfel et al., 2013; Ude et al., 2013). These studies suggested a similar role for elF5A due to the sequence and structural similarity between elF5A and EF-P. Shortly after, Gutierrez and his colleagues (2013) showed that elF5A was also required for the translation of polyproline motifs. Hydroxyl radical mapping experiments revealed that elF5A localized to the $E$ site of ribosome and interacted with the acceptor arm of tRNA on the P site, stimulating the peptidyl-transferase activity of the ribosome (Gutierrez et al., 2013). Therefore, instead of being a global translation factor, elF5A acts during translation of specific proteins. Taken together, these results revealed that elF5A in fact was a functional homolog of EF-P.

elF5A has been associated with several other cellular processes like vesicular trafficking, cell cycle progression, apoptosis and mRNA degradation. It is not yet clear if these are independent functions of elF5A. Based on its recently characterized role in translation, where elF5A can act as a regulator of gene expression of specific proteins, all of these suggested functions might be secondary effects of translation. For the sake of simplicity, I would like to refer the reader to recent reviews discussing these functions (Zanelli and Valentini, 2007; Park et al., 2010; Caraglia et al., 2013).

\subsubsection{Nucleocytoplasmic distribution of elF5A}

As for the function, the subcellular distribution of elF5A had remained controversial for very long time. Throughout the years, several groups reported different subcellular localizations for elF5A (Ruhl et al., 1993; Shi et al., 1996; Rosorius et al., 1999; Jao and Yu Chen, 2002). However, a recent study demonstrated that 
these discrepancies were due to the overexpression of elF5A (Lee et al., 2009). The study showed that endogenous elF5A is localized exclusively to the cytoplasm, whereas exogenous (overexpressed) elF5A displayed both nuclear and cytoplasmic localization. They discovered that overexpressed eIF5A was not completely modified by the endogenous DHS and DOHH. Upon overexpression of these two enzymes, overexpressed elF5A showed cytoplasmic localization. These results also revealed the importance of the hypusine on subcellular localization of elF5A.

With its $17 \mathrm{kDa}$ molecular weight, elF5A is well below the passive diffusion limit of the NPCs, and therefore can diffuse into the nucleus very fast. The exclusive cytoplasmic localization of elF5A is provided by an active export mechanism. Although initially CRM1 was suggested to be the export factor of elF5A (Rosorius et al., 1999), Xpo4 was identified as the export receptor of elF5A (Lipowsky et al., 2000). Xpo4 exports hypusinated as well as deoxyhypusinated and non-modified elF5A. However, the affinity of Xpo4 to the non-modified elF5A is $\sim 35$ fold less than its affinity to the hypusinated-elF5A (Lipowsky et al., 2000). These results might explain why overexpressed elF5A shows mixed localization.

Although, the active import of elF5A to the nucleus has not been characterized so far, elF5A was shown to enrich in the nucleus upon induction of apoptosis by TNF- $\alpha$ (Taylor et al., 2007). Moreover, acetylation of elF5A was recently shown to stimulate nuclear accumulation (Ishfaq et al., 2012). Interestingly, in another study, the nuclear accumulation of elF5A gave rise to cell proliferation, which can be averted by introduction of Xpo4 (Zender et al., 2008). These results might indicate a yet undiscovered nuclear function of elF5A. Alternatively, these results could be experimental artifacts.

\subsection{About this work}

This thesis addresses different aspects of two Imp $\beta$-like NTRs, namely Xpo4 and Xpo7 and therefore the results will be presented in two sections. The first section describes the structural and biochemical investigation of cargo recognition by Xpo4 and forms the major part of my PhD project. The second part constitutes a smaller part of my PhD work where I searched for additional interaction partners of Xpo7. 
In one transport cycle, bidirectional NTRs can carry two cargoes (one import and one export cargo) in the expense of one RanGTP, which makes them very economic compared to the unidirectional NTRs. How Xpo4 (also Imp13 and Msn5p) could work both as importin and exportin was not known at the beginning of my PhD. In order to understand the molecular details of bidirectional transport and to identify the structural aspects of bidirectional NTRs that allow them to operate different than the unidirectional ones, it was necessary to elucidate the cargo-bound as well as cargo-free structures of bidirectional NTRs. For this project, we aimed to solve the structures of RanGTP•Xpo4•elF5A, RanGTP•Xpo4, and Sox2·Xpo4 complexes and the structure of Xpo4 alone by X-ray crystallography. During the course of this project, I have successfully crystallized and solved the structure of the export complex (RanGTP•Xpo4•eIF5A). For this, I first established an in vitro system for the production of high amounts of very pure hypusinated eIF5A. Then, I engineered Xpo4 to obtain diffraction quality crystals. After solving the structure, I validated these findings with biochemical and functional assays. Last part of the first section explains the crystallization trials of the other complexes of Xpo4.

Xpo7 has been suggested to be a broad range exportin like CRM1, however, only three export cargoes have been identified so far. Recent studies by our lab and others have revealed that Xpo7 is required for erythrocyte maturation suggesting that its complete function has not been deciphered. As a result, we decided to elucidate the complete set of Xpo7 binders. By using an optimized affinity chromatography method in combination with mass spectrometry analysis, I have found novel Xpo7 export cargoes. Astonishingly, I also identified several import cargo candidates, which indicated that Xpo7 could be another bidirectional NTR. 


\section{RESULTS}

\subsection{Structural and Biochemical Analysis of Cargo Recognition by Xpo4}

Crystallization of proteins and protein complexes is only possible in a narrow range of multi-dimensional parameters. This necessitates an exhaustive screening for various conditions such as temperature, buffer, precipitant and requires large amounts of proteins or protein complexes. For this project, we needed Xpo4, RanGTP, elF5A as well as Sox2 and decided to produce these proteins recombinantly in E. coli.

For an initial investigation, we used full-length constructs of Xpo4, elF5A and Sox2. Ran was truncated to contain residues $5-180$, excluding the parts that were not structured in the previous NTR-RanGTP structures (Vetter et al., 1999a; Cook et al., 2007). Deletion of C-terminus (residues 181-216) also stabilizes the GTPbound form of Ran and increases the affinity between NTR and RanGTP (Richards et al., 1995; Nilsson et al., 2002). In addition, the Gln69Leu mutation was inserted to prevent GTPase activity of Ran and to stabilize it in GTP-bound form (Bischoff et al., 1994). This construct, RanQ69L ${ }^{5-180}$, will be referred to as RanGTP throughout this section.

Expression and purification of Xpo4, RanGTP and Sox2 had been established in our lab. I further optimized the expression and purification of Xpo4 and RanGTP to improve the solubility as well as purity and to increase the final yield. Details of the latest protocols are described in 5.2.2.

In contrast, there was no established protocol for the production of hypusinecontaining elF5A. Since all of the existing elF5A in eukaryotic cells has hypusine, cellular extracts could be used as a source to purify hypusine-containing elF5A. Lipowsky et al. (2000) used $300 \mathrm{~mL}$ cytoplasmic HeLa extract to obtain $3 \mathrm{mg}$ elF5A. With a similar efficiency, we would have needed tens of liters of cell culture. As a result, this would not have been a cost-efficient method. Moreover, having only the wild type elF5A, use of eukaryotic extracts would not allow the production of truncated or mutated elF5As, which might be required in the later stages of this 
study. These reasons prompted us to use the bacterial expression systems. Nevertheless, recombinantly produced elF5A does not contain hypusine due to lack of DHS and $\mathrm{DOHH}$ in E. coli. Hypusine can be introduced in vivo by co-expressing elF5A with DHS and $\mathrm{DOHH}$ or in vitro by enzymatic reaction. However, it was not clear whether all the produced elF5A would contain hypusine and whether fully modified elF5A could be separable from non-modified and semimodified elF5A species. Consequently, we decided to establish an in vitro system that allows rapid detection of the modification state of elF5A and separation of fully modified elF5A from the other species.

\subsubsection{In vitro elF5A hypusination}

elF5A is posttranslationally modified by $\mathrm{DHS}$ and $\mathrm{DOHH}$ in two consecutive reactions, deoxyhypusination and hydroxylation, as described in section 2.4 and depicted in Figure 2-6. The deoxyhypusination and the hydroxylation reactions have been studied by many groups; therefore, the details of these reactions are well known (reviewed by Park et al., 2010). The deoxyhypusination and the hydroxylation can be used to produce deoxyhypusine-containing elF5A (elF5A(Dhp)) or hypusine-containing elF5A (elF5A(Hpu)) from a bacterially expressed elF5A. However, these reactions reach equilibrium before completion, in other words the efficiency of these reactions, in vitro, is not $100 \%$. As a result, the final reaction mixture contains fully modified, semi-modified as well as non-modified elF5A (elF5A(Lys)). Consequently, it is crucial to separate these differently modified, but otherwise identical proteins. In the following sections, I will explain the methods that led us to separate and purify differently modified elF5As.

\section{The deoxyhypusination}

The deoxyhypusination reaction converts elF5A(Lys) to elF5A(Dhp). There are protocols that employ this reaction in order to produce elF5A(Dhp). The key step is the use of cation exchange chromatography to separate elF5A(Dhp) from elF5A(Lys). Since deoxyhypusination introduces an additional positive charge, elF5A(Dhp) binds the cation exchangers stronger and elutes later in an increasing salt concentration. In order to test this, recombinantly produced elF5A(Lys) was incubated with DHS in the presence of its cofactor NAD and the butylamine donor spermidine in $200 \mathrm{mM}$ glycine $(\mathrm{pH}$ 9.0). As a negative control, I performed the 
same reaction omitting DHS. After $4 \mathrm{~h}$ at $37^{\circ} \mathrm{C}$, the proteins were bound to an SP sepharose column and eluted with a salt gradient. Figure 3-1a shows the elution profiles of different reactions. When DHS was excluded, elF5A was eluted at $\sim 400 \mathrm{mM} \mathrm{NaCl}$. When DHS was present, bound proteins were eluted in two peaks. The first and smaller peak was at $\sim 400 \mathrm{mM} \mathrm{NaCl}$ and the second and larger peak was at $\sim 500 \mathrm{mM} \mathrm{NaCl}$. Both peaks represented elF5A (Figure 3-1b). Therefore, we concluded that the second peak emerged, as predicted, due to the deoxyhypusination and the eluate was elF5A(Dhp). We calculated the efficiency of the reaction to be $90-95 \%$. With this method, we were able to prepare large scale pure elF5A(Dhp).

A

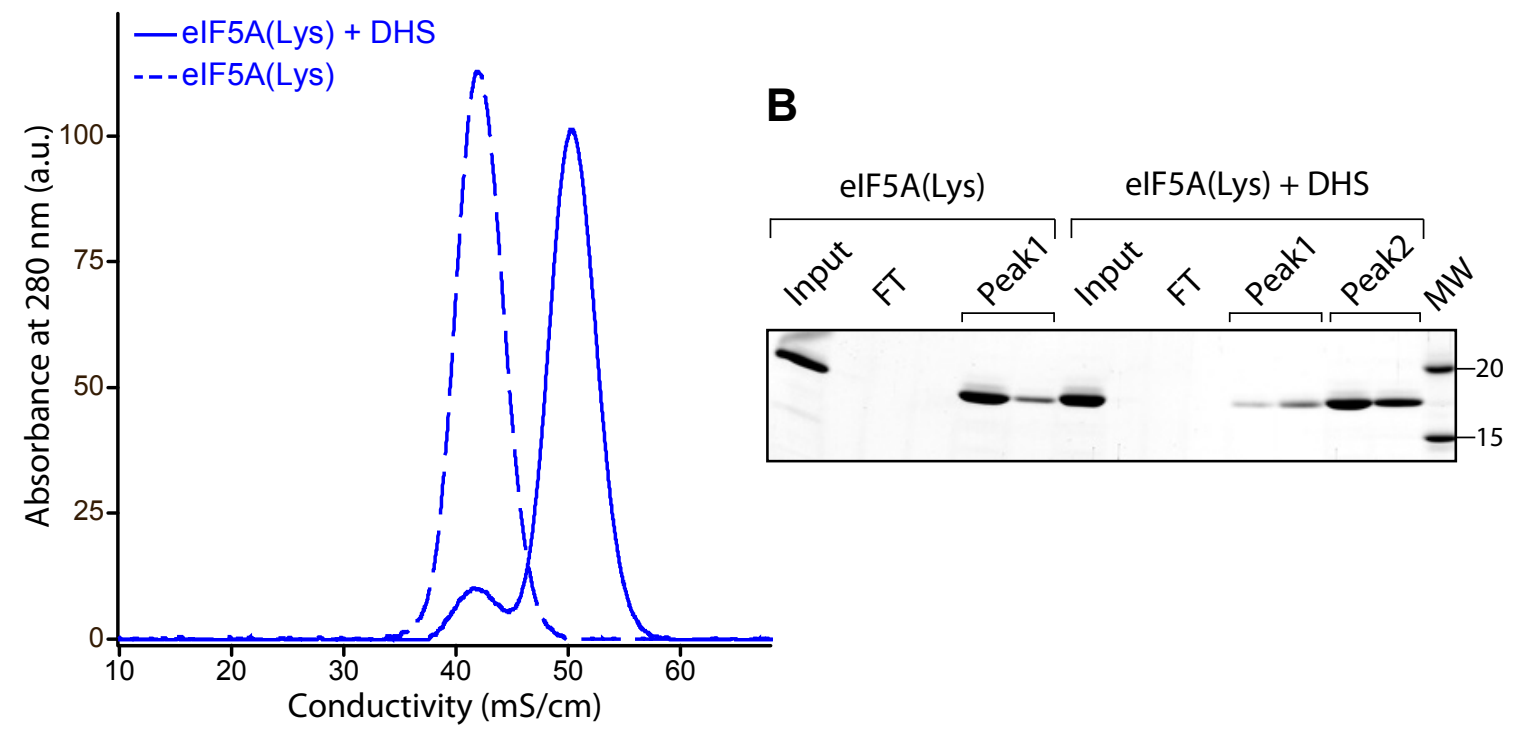

Figure 3-1 in vitro deoxyhypusination of elF5A

$20 \mu \mathrm{M}$ elF5A(Lys) was incubated with $2 \mathrm{mM}$ NAD, $2 \mathrm{mM}$ spermidine, $2 \mathrm{mM}$ DTT in $500 \mu \mathrm{L}$ of $0.2 \mathrm{M}$ glycine $\mathrm{pH}$ 9.0 buffer in the absence or presence of $2 \mu \mathrm{M}$ DHS for $4 \mathrm{~h}$ at $37^{\circ} \mathrm{C}$. After buffer exchange, the samples were loaded to $1 \mathrm{~mL}$ HiTrap SP sepharose column equilibrated with $20 \mathrm{mM}$ potassium phosphate $\mathrm{pH}$ 6.0, $25 \mathrm{mM}$ $\mathrm{NaCl}$ and $2 \mathrm{mM}$ DTT. Bound proteins were eluted with a linear gradient ending at $50 \mathrm{mM}$ potassium phosphate pH 6.0, 0.8 M NaCl and $2 \mathrm{mM}$ DTT (A). Flow through (FT) and peak fractions (Peak1 and Peak2) were collected and analyzed by SDS-PAGE followed by Coomassie blue staining (B). Dashed line and straight line in (A) represent absence and presence of DHS, respectively.

\section{The reversal of the deoxyhypusination and the hydroxylation}

The hydroxylation reaction can be used to convert elF5A(Dhp) to elF5A(Hpu). Unlike the previous situation, there is no chromatographic method that can separate these proteins as they are identical except for an oxygen atom in the elF5A(Hpu). 
In cells, DHS uses spermidine as the butylamine donor and elF5A(Lys) as the butylamine acceptor. In their 2003 study, Park and his colleagues showed that DHS, in vitro, could use deoxyhypusine and other polyamines (i.e. homospermidine) as butylamine donors in the presence of putrescine or 1,3-diaminopropane (DAP), suggesting the reversibility of the deoxyhypusination. A striking result of the study was that the DHS was not able to use elF5A(Hpu) as a donor. I wanted to exploit this specific activity to convert any remaining elF5A(Dhp) after the hydroxylation reaction back to elF5A(Lys), which would leave only elF5A(Hpu) and elF5A(Lys) in the reaction mixture. These can be separated via cation exchange chromatography due to the charge difference between the hypusine and the lysine.

In order to test if the reversal of the deoxyhypusination reaction works, I incubated elF5A(Dhp) with NAD and DAP in the presence or absence of DHS. Then, the products were subjected to cation exchange chromatography. As depicted in Figure 3-2a, elF5A(Dhp) was successfully converted to elF5A(Lys). In the absence of DHS, elF5A(Dhp) was eluted at $\sim 500 \mathrm{mM} \mathrm{NaCl}$ (similar to Figure 3-1). Addition of DHS resulted in elution of elF5A at the same salt concentration as elF5A(Lys) would do. Unlike the deoxyhypusination, the efficiency of the reaction was $\sim 100 \%$. This can be explained by $\sim 150$ fold higher transfer rate of butylamine moiety from elF5A(Dhp) to DHS compared to that from spermidine to DHS (Park et al., 2003).

A



B

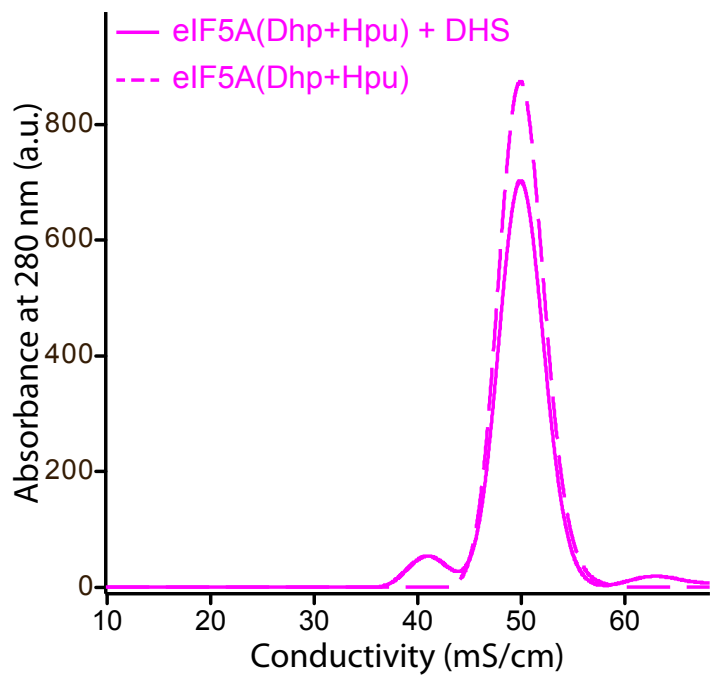

Figure 3-2 The reversal of the deoxyhypusination assays with elF5A(Dhp) and elF5A(Hpu) 
$20 \mu \mathrm{M}$ elF5A was incubated with $2 \mathrm{mM}$ NAD, 2 mM DAP, 2 mM DTT in $500 \mu \mathrm{L}$ of $0.2 \mathrm{M}$ glycine pH 9.0 buffer in the absence or presence of $2 \mu \mathrm{M}$ DHS for $4 \mathrm{~h}$ at $37^{\circ} \mathrm{C}$. After buffer exchange, the samples were loaded to $1 \mathrm{~mL}$ HiTrap SP sepharose column equilibrated with $20 \mathrm{mM}$ potassium phosphate $\mathrm{pH} 6.0,25 \mathrm{mM} \mathrm{NaCl}$ and 2 $\mathrm{mM}$ DTT. Bound proteins were eluted with a linear gradient ending at $50 \mathrm{mM}$ potassium phosphate $\mathrm{pH} 6.0$, $0.8 \mathrm{M} \mathrm{NaCl}$ and $2 \mathrm{mM}$ DTT.

(A) elF5A(Dhp) was subjected to the reversal of the deoxyhypusination assay. (B) $20 \mu \mathrm{M}$ elF5A(Dhp) was incubated with $20 \mu \mathrm{M} \mathrm{DOHH}$ and $2 \mathrm{mM}$ DTT in $500 \mu \mathrm{L}$ of $25 \mathrm{mM}$ Tris/HCl pH7.5 buffer for $4 \mathrm{~h}$ at $37^{\circ} \mathrm{C}$. The reaction products were split into two and used in the reversal of the deoxyhypusination assay. Dashed lines and straight lines represent absence and presence of DHS, respectively.

After observing that the reversal of the deoxyhypusination reaction works, the next was to test the products of the hydroxylation reaction. I first carried out the hydroxylation reaction with elF5A(Dhp) and $\mathrm{DOHH}$. Then, the products were split into two and only one of the samples was incubated with DHS for reversal. The products were analyzed by cation exchange chromatography as used before. The elution profile of the portion that did not have the DHS was similar to that of elF5A(Dhp) (Figure 3-2b), as expected elF5A(Dhp) and elF5A(Hpu) were not separated. In contrast, another peak emerged $(\sim 40 \mathrm{mS} / \mathrm{cm})$ in the DHS containing sample corresponding to elF5A(Lys). Given that the efficiency of the reversal of the deoxyhypusination reaction was $100 \%$, we concluded that the peak at 50 $\mathrm{mS} / \mathrm{cm}$ resembled only elF5A(Hpu).

After confirming that the hypusine could be introduced in vitro and hypusinated elF5A can be separated from the others effectively, I used the system depicted in Figure 3-3 to produce large scale elF5A(Hpu). Briefly, recombinant elF5A was modified by DHS and DOHH to form deoxyhypusine and hypusine. Non-modified elF5A was separated from the rest by cation exchange chromatography. Afterwards, the deoxyhypusine was converted to the lysine and elF5A(Lys)elF5A(Hpu) mixture was subjected to cation exchange chromatography to obtain pure hypusinated eIF5A. 


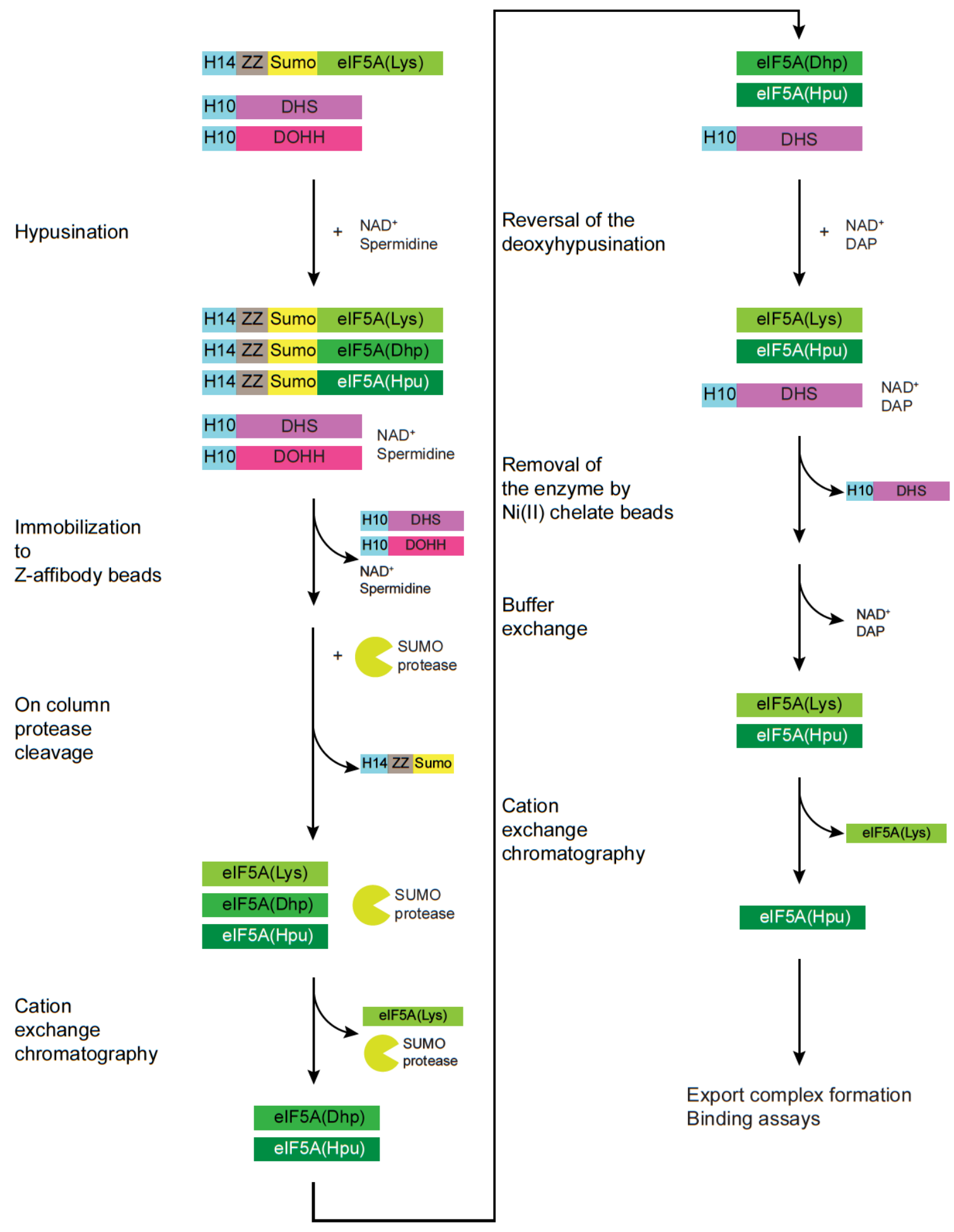

Figure 3-3 Scheme for large scale elF5A(Hpu) purification

\section{Assessing the effect of the modifications on Xpo4 binding}

Although the hypusination is not absolutely essential for the elF5A Xpo4•RanGTP interaction, each modification increases the affinity of the elF5A to Xpo4•RanGTP complex (75 nM, $25 \mathrm{nM}$, and $2 \mathrm{nM}$ respectively from lysine to hypusine; Lipowsky 
et al., 2000). I performed the following experiment to test the binding strength of different elF5A variants to Xpo4•RanGTP. First, each elF5A variant was allowed to form export complexes with RanGTP and Xpo4 in solutions containing various salt concentrations. Then, complexes were recovered on phenyl sepharose beads via Xpo4. Finally, the bound proteins were eluted with SDS sample buffer and analyzed by SDS-PAGE (Figure 3-4). Although the amount of the bound elF5A gradually decreased in all samples as the salt concentration increases, the bound elF5A was significantly higher in the hypusinated version and the effect of salt was less prominent in elF5A(Hpu) compared to the other two variants.

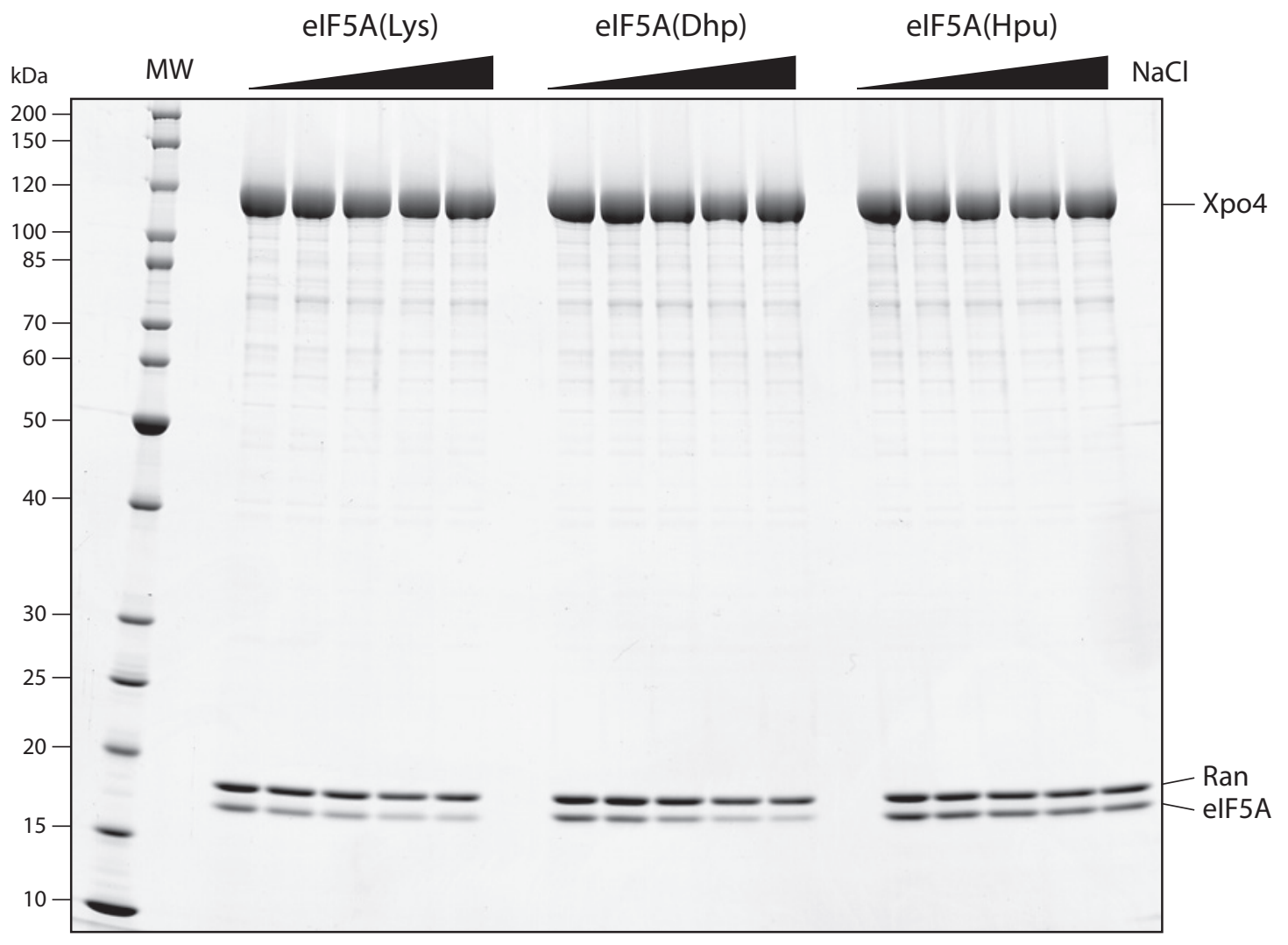

Figure 3-4 Salt sensitivity of the elF5A variants

The samples were prepared by mixing $0.75 \mu \mathrm{M}$ elF5A variants with $0.75 \mu \mathrm{M}$ RanGTP and $0.5 \mu \mathrm{M}$ Xpo4 in $350 \mu \mathrm{L}$ of $50 \mathrm{mM}$ Tris/ $\mathrm{HCl}, 2 \mathrm{mM} \mathrm{Mg}(\mathrm{OAc})_{2}$ buffer with various salt concentrations $(50,100,150,200$, and 250 $\mathrm{mM} \mathrm{NaCl}$ ). After $2 \mathrm{~h}$ incubation at $4{ }^{\circ} \mathrm{C}$, Xpo4 was immobilized to phenyl sepharose beads. Unbound proteins were removed by washing with buffer of the corresponding salt concentration. Xpo4 and bound proteins were eluted with SDS. The eluates were analyzed by SDS-PAGE followed by Coomassie blue staining. 'MW' stands for molecular weight marker, and protein sizes are marked on the left. 


\subsubsection{Crystallization of the elF5A export complex}

\section{Reconstitution of elF5A export complex}

After producing the hypusinated elF5A, I proceeded to form the elF5A export complex. I mixed elF5A(Hpu) and Xpo4 with H14-ZZ-bdSUMO tagged RanGTP in near stoichiometric ratio, elF5A(Hpu) being in excess in order to saturate the RanGTP. After incubating the mixture at $4{ }^{\circ} \mathrm{C}$ for $3 \mathrm{~h}$, the complex was pulled down via H14-ZZ-bdSUMO RanGTP by immobilizing to anti-Z affibody dimer coupled beads. Afterwards, unbound proteins were removed by washing and the complex was eluted with bdSUMO protease. The eluate was analyzed by size exclusion chromatography followed by SDS-PAGE (Figure 3-5a, b). Injected proteins were eluted as a single peak and this peak contained all the components of the export complex. In order to determine the absolute mass and the stoichiometry of the complex, peak fractions of the gel filtration were pooled, concentrated and subjected to a Superdex 200 10/30 gel filtration column coupled to a multi angle light scattering (MALS) detector (Figure 3-5c). The detected molecular mass of $\sim 167 \mathrm{kDa}$ was fully consistent with a 1:1:1 stoichiometric RanGTP•Xpo4•elF5A(Hpu) complex. The theoretical mass of the export complex is also $167 \mathrm{kDa}(20 \mathrm{kDa}(\operatorname{Ran})+130 \mathrm{kDa}(\mathrm{Xpo} 4)+17 \mathrm{kDa}(\mathrm{elF} 5 \mathrm{~A}))$.

In the crystallization facility of MPI-BPC, I tried to crystallize the elF5A export complex using commercially available crystallization screens and tested $\sim 2500$ different conditions. Nevertheless, none of the tested conditions gave rise to crystals or crystalline-like substances.

Flexible regions in the proteins have been considered to be one of the reasons for failures in crystallization. Therefore, I tried to find out possible flexible/disordered regions in the proteins. I was already using the truncated version of Ran that was missing the flexible regions both in the $\mathrm{N}$ - and $\mathrm{C}$-termini. Prediction algorithms identified N-terminal of elF5A to be disordered (Figure 3-6). In fact, first 14 amino acid residues were not visible in the crystal structure of yeast elF5A, and these residues (and the last three amino acids) had to be removed in order to crystallize the human elF5A (Tong et al., 2009). 
A

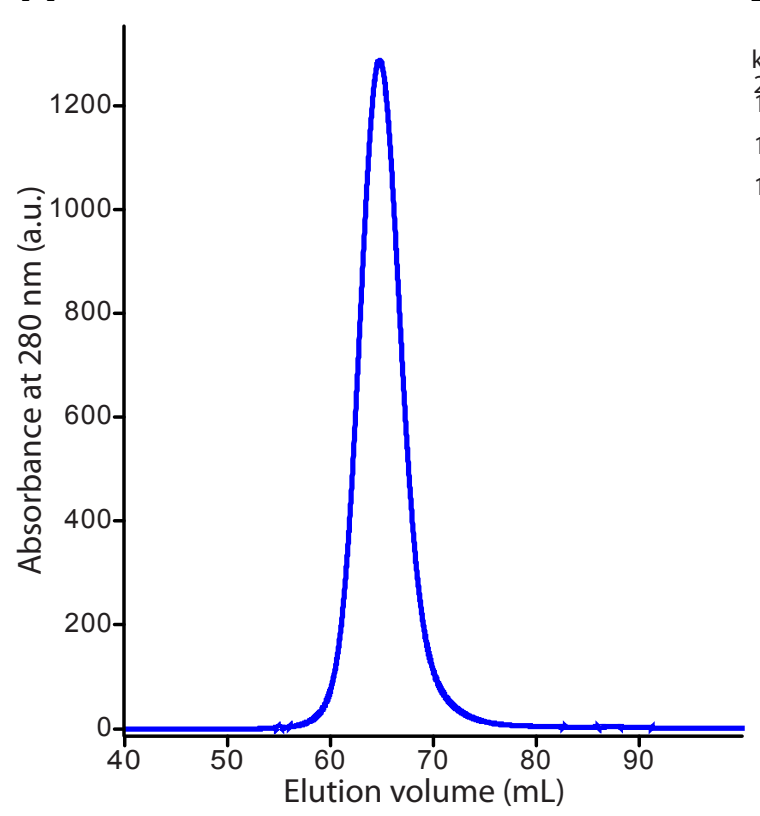

B

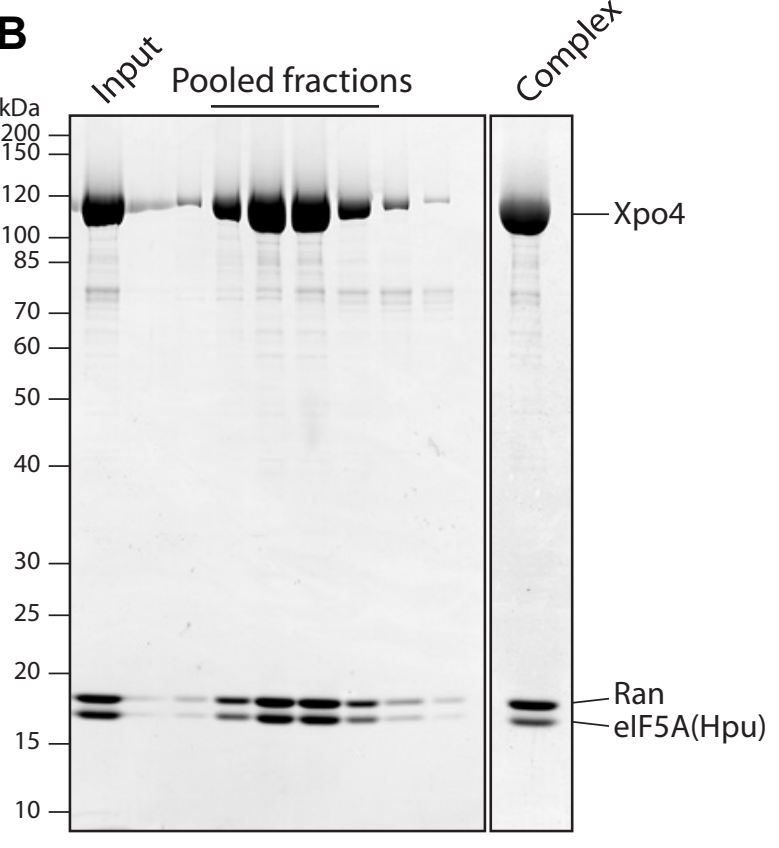

C

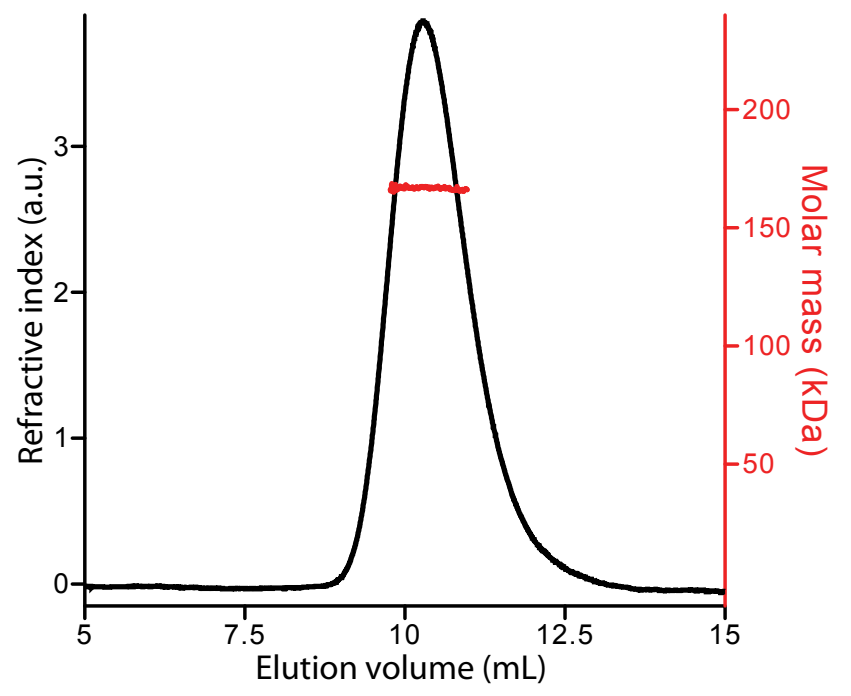

Figure 3-5 Reconstitution of elF5A export complex

(A) Gel filtration profile of the export complex on Superdex $20016 / 60$ column equilibrated with $15 \mathrm{mM}$ Tris/ $\mathrm{HCl}$ $\mathrm{pH} 7.7,18 \mathrm{mM} \mathrm{NaCl}, 2 \mathrm{mM} \mathrm{Mg}(\mathrm{OAc})_{2}$ and $2 \mathrm{mM}$ DTT. Proteins were collected in $1.5 \mathrm{~mL}$ fractions, pooled and concentrated to $12 \mathrm{mg} / \mathrm{mL}$ (Complex). (B) $5 \mu \mathrm{L}$ of the fractions and $3 \mu \mathrm{g}$ of the "input" and the "complex" were analyzed by SDS-PAGE followed by Coomassie blue staining. (C) Complex was diluted to $2 \mathrm{mg} / \mathrm{mL}$ and analyzed by Superdex 200 10/30 column coupled to MALS detector. Theoretical molar mass of a 1:1:1 stoichiometric complex is $167 \mathrm{kDa}$ that matches exactly with the detected mass. 


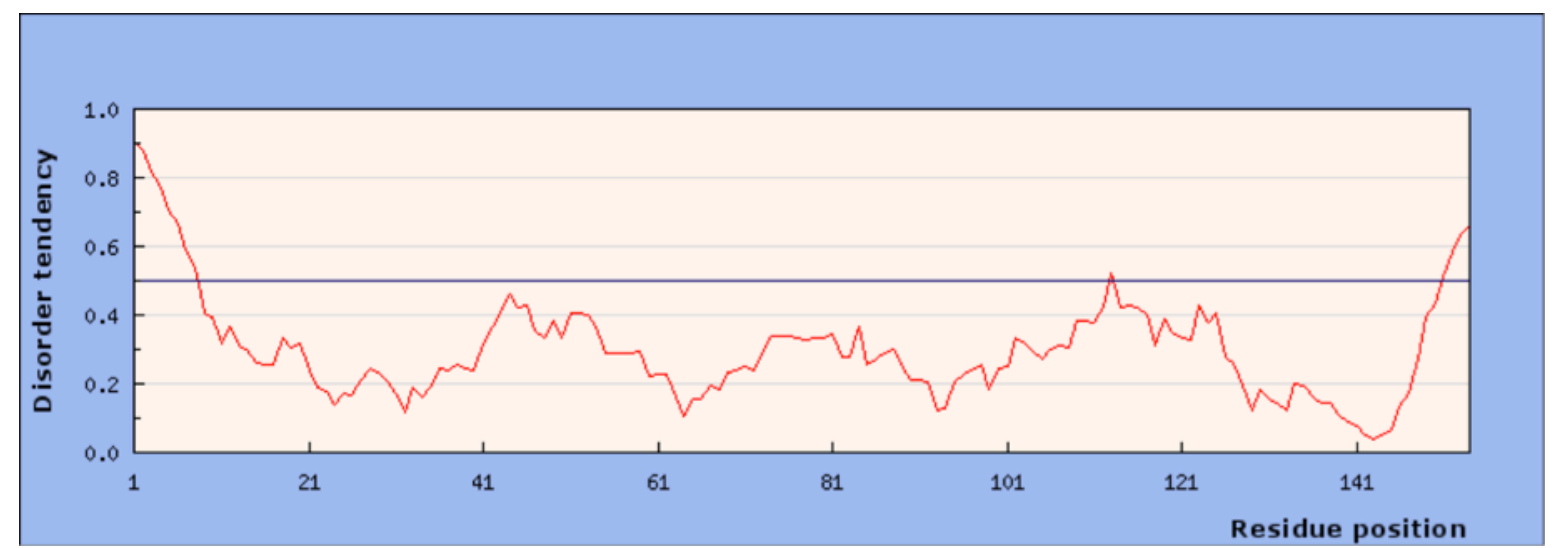

\section{Figure 3-6 Disorder prediction of elF5A}

Primary sequence of elF5A was analyzed by IUPred website using the settings to search for short disordered regions. Predicted disorder score of each amino acid was plotted against the amino acid position in the primary sequence. Values greater than 0.5 indicates unstructured regions.

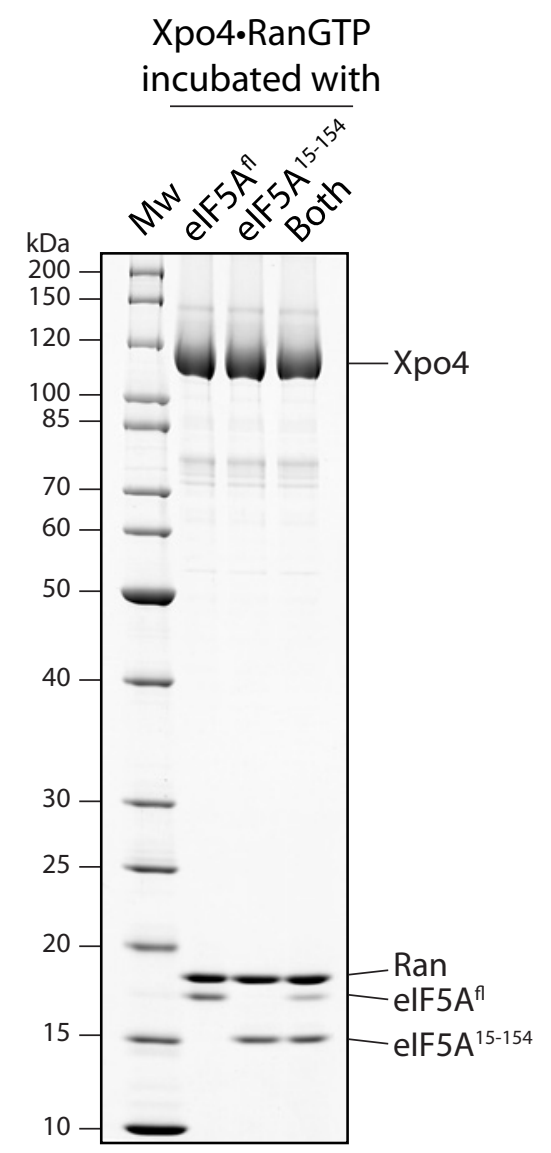

Figure 3-7 Binding of truncated elF5A to Xpo4-RanGTP

The samples were prepared by mixing $0.5 \mu \mathrm{M}$ elF5A(Hpu) with $0.6 \mu \mathrm{M}$ RanGTP and $0.5 \mu \mathrm{M}$ Xpo4 in $350 \mu \mathrm{L}$ of $50 \mathrm{mM}$ Tris/ $\mathrm{HCl}, 30 \mathrm{mM} \mathrm{NaCl}, 2 \mathrm{mM} \mathrm{Mg}(\mathrm{OAc})_{2}$ and $2 \mathrm{mM} \mathrm{DTT}$. After $2 \mathrm{~h}$ incubation at $4{ }^{\circ} \mathrm{C}, 300 \mu \mathrm{L}$ of the samples was incubated with phenyl sepharose beads to precipitate Xpo4 and bound proteins. Unbound proteins were removed; Xpo4 and the bound proteins were eluted with SDS sample buffer. The eluates were analyzed by SDS-PAGE followed by Coomassie blue staining. For competition assay, $0.5 \mu \mathrm{M}$ of each of the full-length and truncated elF5A(Hpu) were mixed with RanGTP and Xpo4. 'MW' and 'fl' stand for molecular weight marker and full-length, respectively. 
I created a truncated version of elF5A (elF5A ${ }^{15-154}$ ) and wanted to test if a complex composed of elF5A ${ }^{15-154}$ would crystallize. However, I first had to check if the truncated version would be hypusinated and form complex with Xpo4 and RanGTP. In vitro hypusination system that I established for full-length elF5A effectively worked for elF5 $A^{15-154}$ as well. After producing hypusinated elF5A ${ }^{15-154}$, I assessed its ability to bind Xpo4 and RanGTP. Figure 3-7 shows the result of the binding assay of the full-length and the truncated elF5A(Hpu) as well as a competition experiment. elF5A ${ }^{15-154}$ was able to form a complex with Xpo4 and RanGTP. The remarkable result was that the shorter version was able to compete out the full-length version in the competition assay (Lane 3 in Figure 3-7) indicating that the truncation did not cause any decrease in the affinity of elF5A to Xpo4•RanGTP.

I prepared an export complex with elF5 $\mathrm{A}^{15-154}$, Xpo4 and RanGTP the same way I did for the full-length elF5A, and analyzed the complex by size exclusion chromatography, SDS-PAGE and MALS (Figure 3-8). All proteins were present in the eluate of the protease elution and ran together in the gel filtration. MALS analysis showed that the absolute mass of the complex was $\sim 163 \mathrm{kDa}$ confirming the shortening of the eIF5A.

I set crystallization drops with the new complex and tested $\sim 1200$ different conditions. Unfortunately, I did not get any three dimensional crystals. Nevertheless, I obtained needle like crystals or sea urchin like needle clusters in three different conditions (Figure 3-8 and Table 3-1).

\begin{tabular}{|c|c|c|c|c|}
\hline $\begin{array}{l}\text { Screen } \\
\text { name }\end{array}$ & $\begin{array}{l}\text { Drop } \\
\text { position }\end{array}$ & Precipitant composition & $\begin{array}{l}\text { Incubation } \\
\text { temperature }\end{array}$ & $\begin{array}{l}\text { Protein } \\
\text { concentration }\end{array}$ \\
\hline PEG II & $\mathrm{A} 2$ & $\begin{array}{l}0.1 \mathrm{M} \text { MES pH } 6.5 \\
15 \% \text { PEG } 400\end{array}$ & $20^{\circ} \mathrm{C}$ & $12 \mathrm{mg} / \mathrm{ml}$ \\
\hline Nucleix & E7 & $\begin{array}{l}0.05 \mathrm{M} \text { Succinic acid pH } 5.5 \\
10 \% \text { 2-Propanol } \\
0.01 \mathrm{M} \mathrm{MgCl}_{2} \\
0.002 \mathrm{M} \mathrm{CoCl}_{2} \cdot 6 \mathrm{H}_{2} \mathrm{O}\end{array}$ & $4^{\circ} \mathrm{C}$ & $12 \mathrm{mg} / \mathrm{ml}$ \\
\hline Nucleix & A10 & $\begin{array}{l}0.05 \mathrm{M} \mathrm{MES} \mathrm{pH} 6.0 \\
5 \% \mathrm{PEG} 4000 \\
0.01 \mathrm{M} \mathrm{MgSO}_{4}\end{array}$ & $20^{\circ} \mathrm{C}$ & $12 \mathrm{mg} / \mathrm{ml}$ \\
\hline
\end{tabular}

Table 3-1 Crystallization conditions of the initial hits 

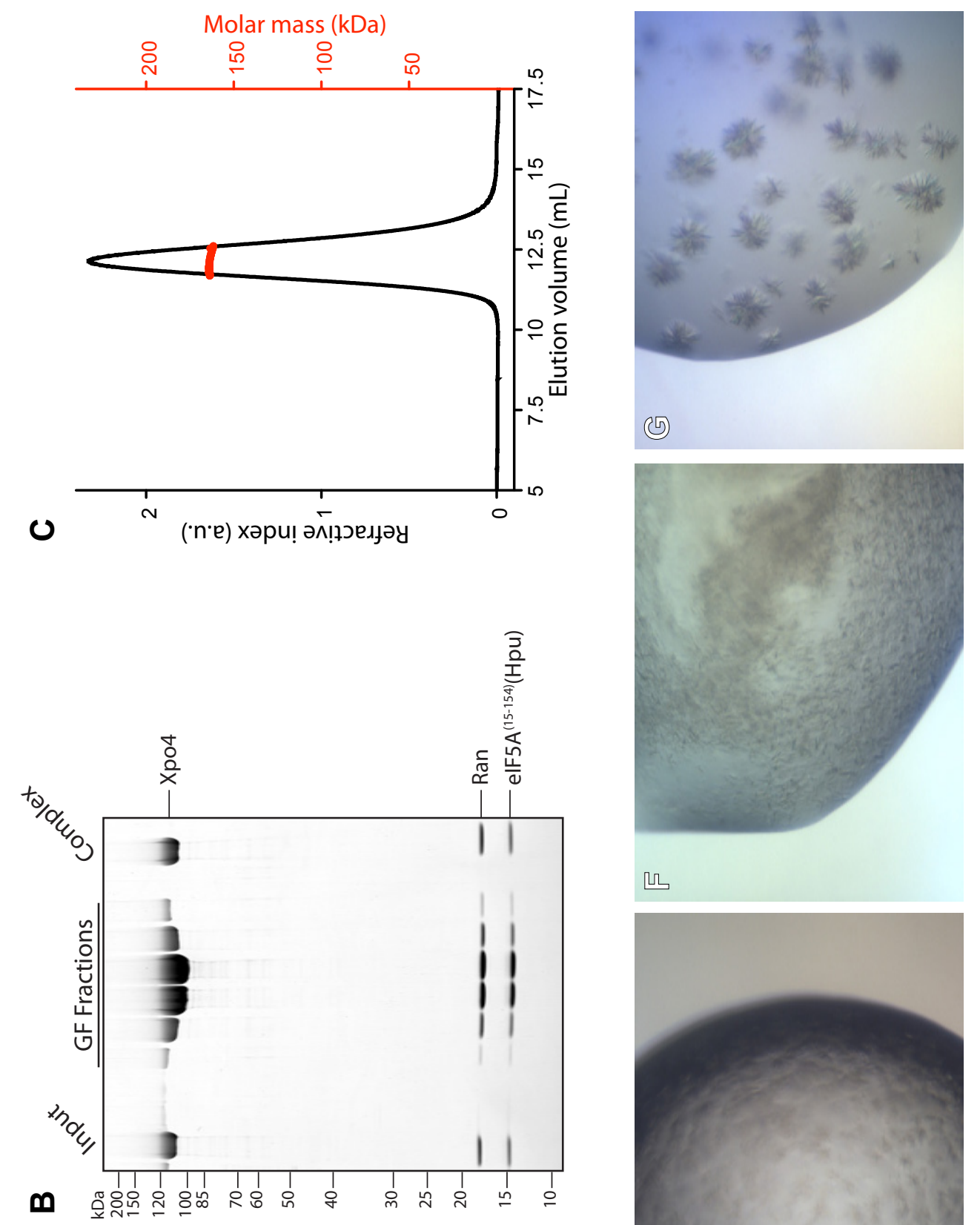

凹
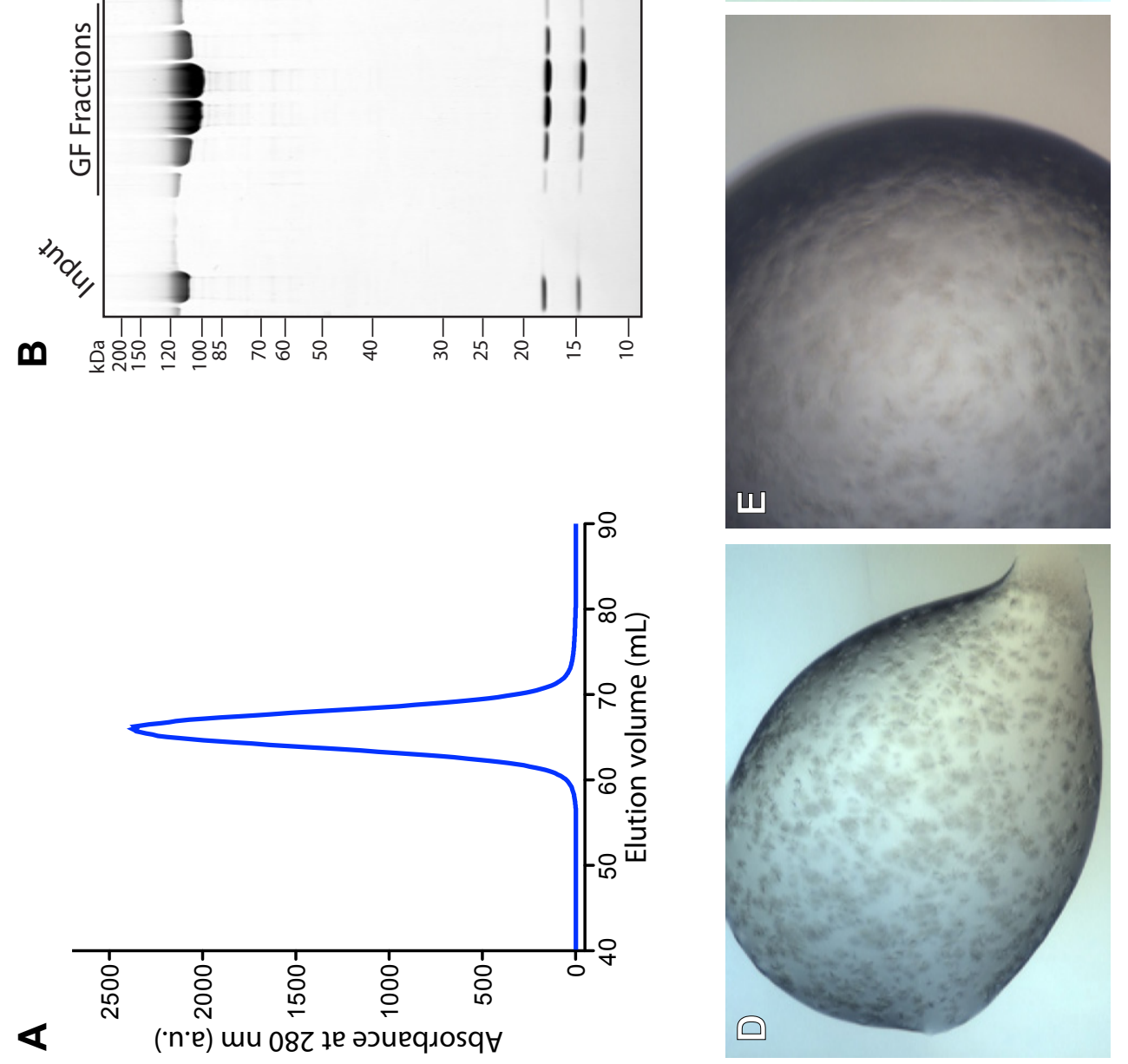
Figure 3-8 Reconstitution of elF5A15-154 export complex and the crystallization hits

(A) Gel filtration profile of the truncated elF5A-containing export complex on Superdex $20016 / 60$ column equilibrated with $15 \mathrm{mM}$ Tris/ $\mathrm{HCl} \mathrm{pH} 7.7,18 \mathrm{mM} \mathrm{NaCl}, 2 \mathrm{mM} \mathrm{Mg}(\mathrm{OAc})_{2}$ and $2 \mathrm{mM}$ DTT. Proteins were collected in $1.5 \mathrm{~mL}$ fractions, pooled and concentrated to $12 \mathrm{mg} / \mathrm{mL}$ (Complex). (B) $5 \mu \mathrm{L}$ of the fractions and $2 \mu \mathrm{g}$ of the "input" and the "complex" were analyzed by SDS-PAGE followed by Coomassie blue staining. (C) Complex was diluted to $2 \mathrm{mg} / \mathrm{mL}$ and analyzed by Superdex 200 10/30 column coupled to MALS detector. Theoretical molar mass of a 1:1:1 stoichiometric complex is $165 \mathrm{kDa}$. (D, E, F, G) Export complex crystals in various crystallization conditions. (D) PEG II-A2, (E) Nucleix-E7, (F) Nucleix A10. For drop compositions see Table 3-1. (G) $0.1 \mathrm{M}$ MES pH 6.5, 13.5\% PEG400. This was the most promising crystallization condition after the initial refinement screen. Sea urchin-like needle clusters appeared after $12 \mathrm{~h}$ and grew full size in 3 days. This condition was used as basis for further refinements.

Clusters or small needles are not suitable for data collection in the synchrotron. Consequently, I tried to optimize the crystallization conditions to obtain diffraction quality crystals. I initially tested conditions with varying $\mathrm{pH}$ and precipitant concentration (Figure 5-1). Later, I assessed the effects of different salts, precipitants, buffers, incubation temperatures and protein concentrations. Additionally, I tested additive screens and silver bullets. Although I observed crystals (needle clusters) as soon as $12 \mathrm{~h}$ (Figure 3-8g), none of the tested conditions improved the shape of the crystals.

\section{Identification of unstructured regions of Xpo4}

Formation of the needle like crystals and the failure of the optimization led us to hypothesize that the removal of the N-terminus of elF5A allowed the complex to make stable crystal contacts in one dimension; nonetheless, other flexible regions were blocking additional crystal contacts that would turn the needles to $2 \mathrm{D}$ or $3 \mathrm{D}$ crystals. At this stage, we were using the minimal RanGTP and elF5A that lack any flexible regions; thus, Xpo4 would have been the only source of the flexibility.

As described in section 2.3, NTRs are made up of successive alpha helices that are connected by loops in different sizes. We suspected, as in the case of yeast CRM1 (Koyama and Matsuura, 2010), Xpo4 might have long insertions that have to be removed to improve the crystals. To test this idea, I decided to employ the limited proteolysis technique. The principle of the technique is that the protein or protein complex is incubated with trace amount of proteases (endopeptidases) so that the partly folded or flexible regions are cleaved while the folded domains remain intact. After the incubation, the samples are analyzed by SDS-PAGE and the emergence of lower molecular weight fragments imply digestions of the fulllength protein, and hence the presence of flexible loops. 


\section{In vitro limited proteolysis}

I performed in vitro limited proteolysis experiments with the export complexes consisting of either full-length or truncated elF5A. I used three proteases; namely trypsin, chymotrypsin and GluC to cover a broad range of amino acids. Trypsin cleaves after the positively charged residues, GluC after the negatively charged residues (preferentially glutamic acid) and chymotrypsin after the bulky hydrophobic residues.

I prepared digestion mixtures (consisting of the protease and the export complexes) with various protease concentrations. The mixtures were incubated for $1 \mathrm{~h}$ at $20^{\circ} \mathrm{C}$. The reactions were stopped by mixing the samples with EDTA and PMSF supplemented SDS sample buffer and incubating $5 \mathrm{~min}$ at $95{ }^{\circ} \mathrm{C}$. The samples were analyzed by SDS-PAGE (Figure 3-9).

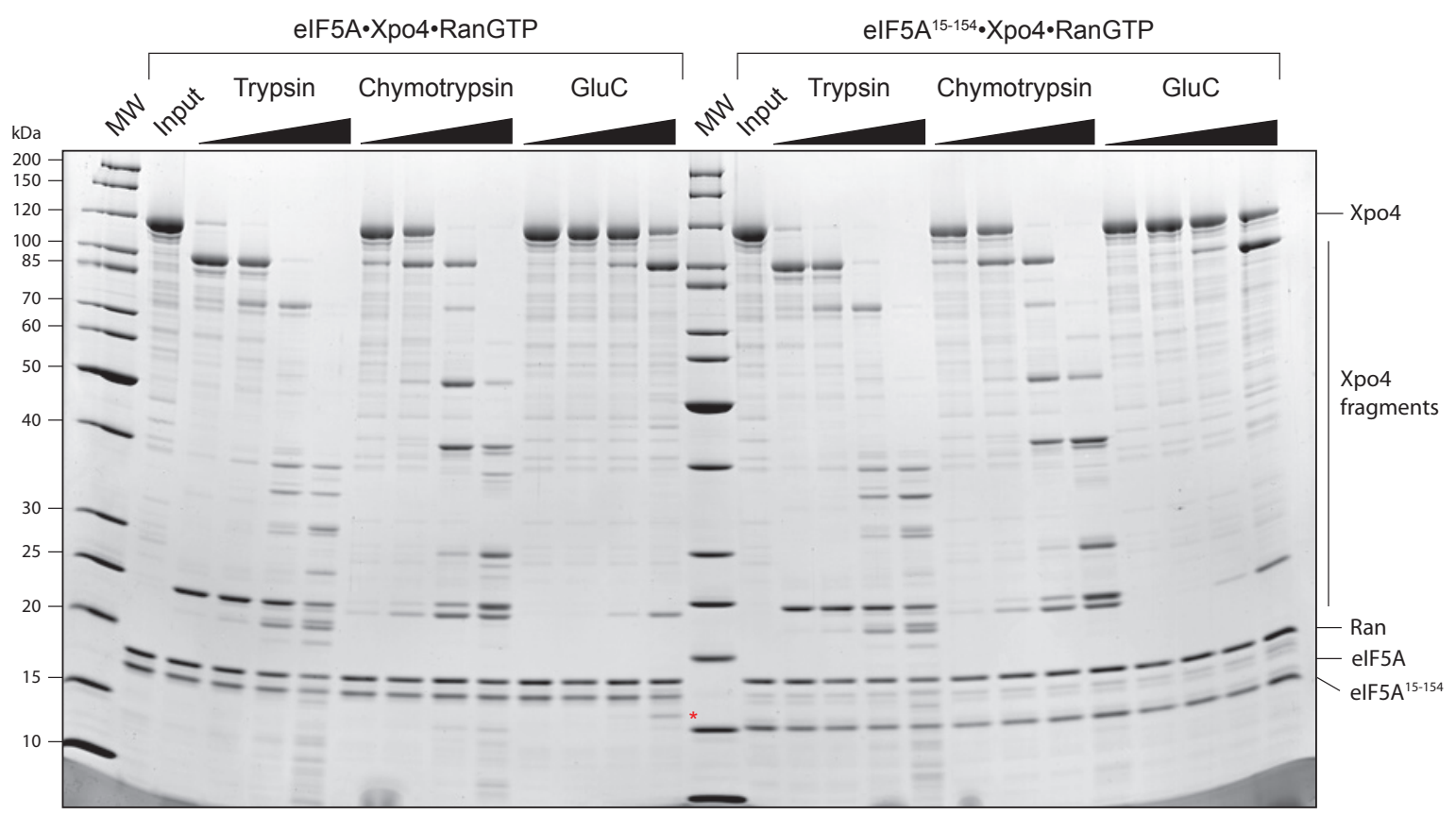

Figure 3-9 in vitro limited proteolysis of elF5A export complexes

$3 \mu \mathrm{g}$ of the export complexes were incubated with increasing concentrations $(1: 2500,1: 500,1: 100$ and 1:20 $\mathrm{w} / \mathrm{w}$ protease to substrate ratio, respectively) of trypsin, chymotrypsin and gluC for $1 \mathrm{~h}$ at $22^{\circ} \mathrm{C}$. The samples were analyzed by SDS-PAGE followed by Coomassie blue staining. As a control (input), $3 \mu \mathrm{g}$ from each complex was prepared and incubated together with the digestions. MW stands for molecular weight marker. $\left.{ }^{*}\right)$ marks the fragment that is the only difference in the digestion patterns of different complexes.

Xpo4, although at varied extents, was susceptible to all of the tested proteases. Digestion pattern of Xpo4 was similar for both complexes, suggesting that the removal of $\mathrm{N}$-terminus of elF5A did not have any conformational change in Xpo4. The only difference between the digestion pattern of both complexes was the presence of a $\sim 16 \mathrm{kDa}$ band in the GluC digestion of the full-length elF5A export 
complex (Figure 3-9 red asterisk). The loss of the same band in the other complex suggested that it belonged to elF5A; and since similar low molecular weight product was missing in the shorter complex, I assumed that the digestion happened in the $\mathrm{N}$-terminal region of elF5A. Finally, it should be noted that among the tested proteases trypsin was the most efficient while the GluC was the least.

The remarkable outcome of the experiment was the appearance of Xpo4 fragments at $\sim 25 \mathrm{kDa}$ and $\sim 100 \mathrm{kDa}$ in all proteases. This implied that Xpo4 had one or more of the flexible loops that were prone to cleavage by the tested proteases. There were at least two prominent bands between 20 and $25 \mathrm{kDa}$ in trypsin and chymotrypsin digestions, on the other hand, there was a single band in GluC. This might be due to lower activity of GluC compared to the others or Xpo4 might have two flexible loops that only one of them is the target of GluC. After discovering that Xpo4 might have flexible or partially folded regions, I performed in situ limited proteolysis in order to test whether the disruption of these loops would improve the quality of the crystals. In the following section, I will explain the identified protease cleavage sites in order to make the subsequent sections comprehensible.

\section{Identification of the protease cleavage sites by mass spectrometry}

The aim of titrating the protease concentrations in the limited proteolysis experiment was to observe the possible digestion intermediates. It was possible to observe such intermediates in trypsin and chymotrypsin digestions (Figure 3-9). The bands corresponding to Xpo4 fragments were excised (including the $\sim 16 \mathrm{kDa}$ band in GluC digestion) from the polyacrylamide gel and analyzed by mass spectrometry.

We were able to identify the composition and the protease cleavage sites of the indicated fragments in Figure 3-10. The peptide sequences that are used to locate the protease cleavage sites are displayed in Table 6-2. We identified that Xpo4 was cleaved by trypsin at Arg245 and Arg947; by chymotrypsin at Tyr247, Phe518 and Phe936; and by GluC at Glu934. As can be recognized, different proteases cleaved very close regions implying that $\mathrm{Xpo} 4$ had at least three unstructured regions or large loops. These were at the $\mathrm{N}$-terminus ( 250 residue), C-terminus ( $\sim 40$ residue) and at the middle ( 520 residue) of Xpo4 (Figure 3-10b). 


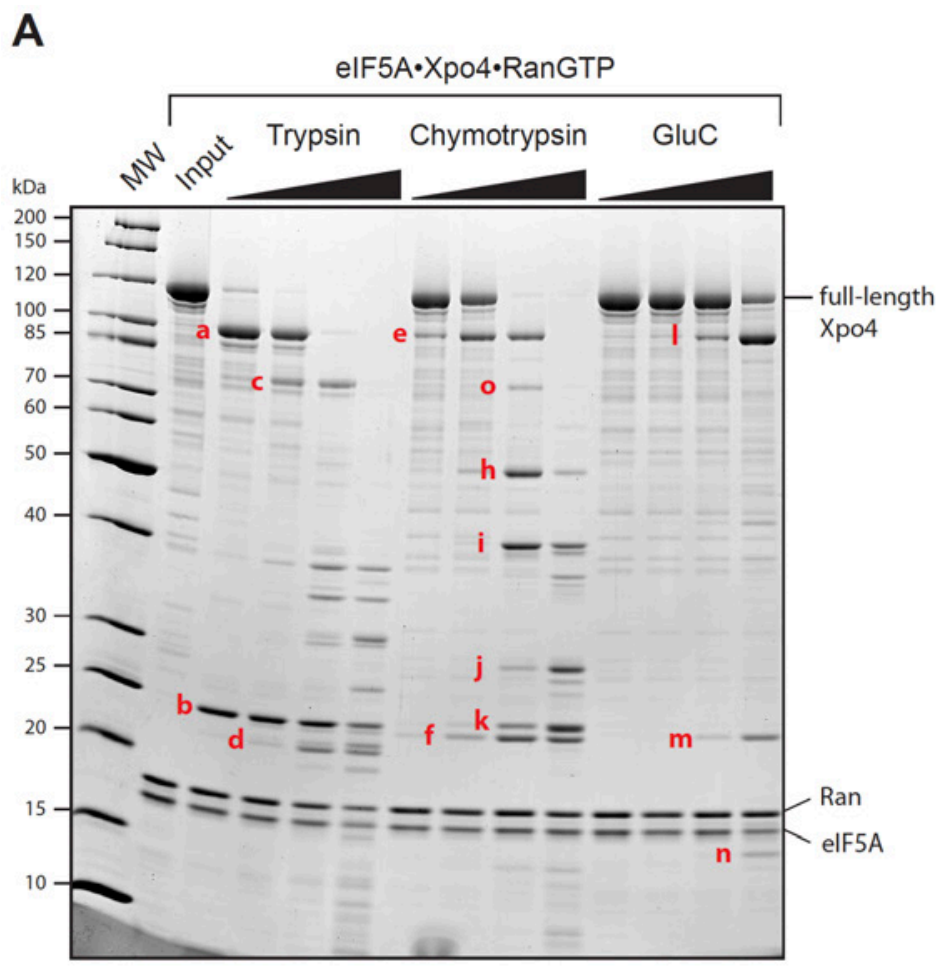

B

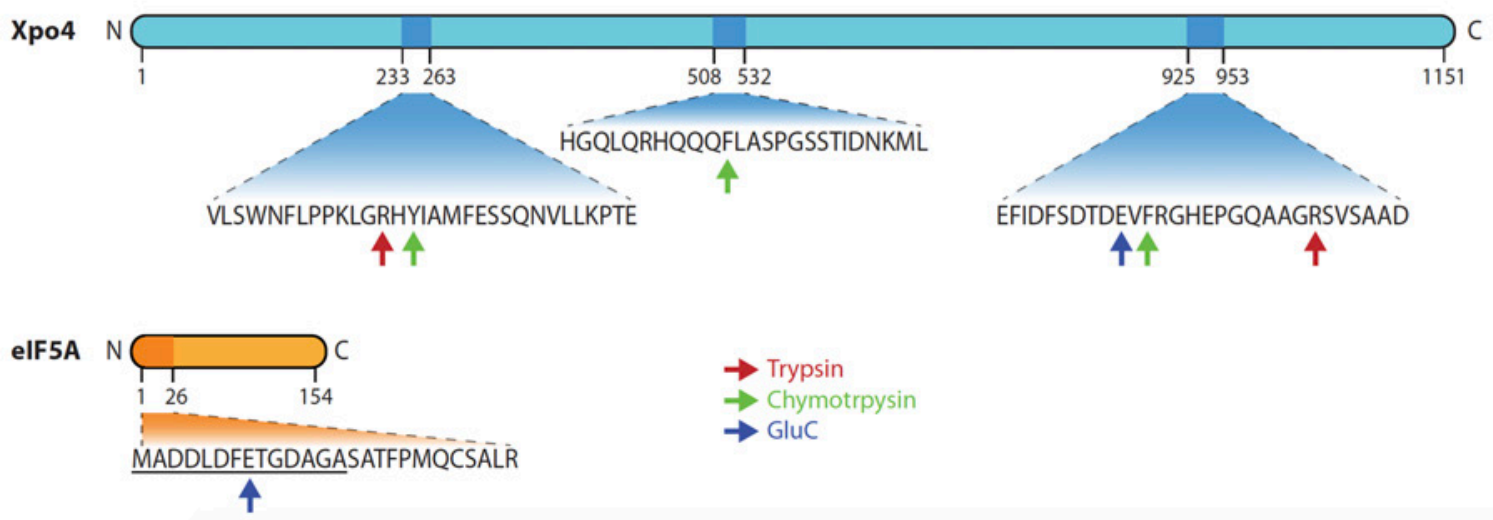

Figure 3-10 Identification of the protease cleavage sites

(A) Digestion fragments that were analyzed by MS are marked with bold letters. (B) Schematic representation of Xpo4 (cyan) and elF5A (orange). The regions that are susceptible to proteases are highlighted and the corresponding sequences are shown beneath. Red, green and blue arrows mark the positions of the cut sites of trypsin, chymotrypsin and GluC, respectively. Underlined residues in elF5A represent the amino acids that were deleted to obtain the needle clusters.

Moreover, as predicted, fragment ' $n$ ' belonged to elF5A. We identified elF5A peptides after the Glu8, suggesting this was the GluC cut site. This result showed that $\mathrm{N}$-terminal of elF5A, in fact, was not structured in the export complex and nicely correlated with the previous findings. 


\begin{tabular}{|lll|}
\hline Protease & Identified peptide & $\begin{array}{l}\text { Corresponding fragment } \\
\text { in Figure 3-10a }\end{array}$ \\
\hline Trypsin & ${ }^{230}$ ANQVLSNFLPPNLGR ${ }^{245}$ & $\mathrm{~b}$ \\
\hline Trypsin & ${ }^{246}$ HYIAMFESSQNVLKPTESWR $^{265}$ & $\mathrm{a}$ \\
\hline Trypsin & ${ }^{948}$ SVSAADVVLY $^{957}$ & $\mathrm{~d}$ \\
\hline Chymotrypsin & ${ }^{925}$ EFIDFSGTDEVF $^{936}$ & $\mathrm{e}$ \\
\hline Chymotrypsin & ${ }^{937}$ RGHEPGQAAGR $^{948}$ & $\mathrm{f}$ \\
\hline Chymotrypsin & ${ }^{248}$ IAMFESSQNVLLKPTESWR $^{266}$ & $\mathrm{o}$ \\
\hline Chymotrypsin & ${ }^{236}$ SWNFLPPNLGR $^{246}$ & $\mathrm{k}$ \\
\hline Chymotrypsin & ${ }^{520}$ ASPGSSTIDNKML $^{532}$ & $\mathrm{~h}$ \\
\hline Chymotrypsin & ${ }^{508}$ HGQLQRHQQQF $^{518}$ & $\mathrm{i}$ \\
\hline GluC & ${ }^{925}$ EFIDFSGTDE $^{934}$ & $\mathrm{l}$ \\
\hline GluC & ${ }^{935}$ VFRGHEPGQAAGR $^{947}$ & $\mathrm{~m}$ \\
\hline GluC & ${ }^{9}$ TGDAGASATFPMQCSALR $^{27}$ & $\mathrm{n}(\mathrm{elF5A})$ \\
\hline
\end{tabular}

Table 3-2 Identified peptides that are used to determine the protease cleavage sites

Identified peptides at the very $\mathrm{N}$ - or $\mathrm{C}$-termini of the corresponding fragments are shown. These peptides were used to identify the protease cleavage sites and can be used as a reference for Figure 3-10b.

\section{In situ limited proteolysis}

After discovering that Xpo4 had flexible or partially folded regions, I performed in situ limited proteolysis in order to test if the disruption of these loops would improve the shape of the crystals. I first determined the rate of the proteolysis in the precipitant $(0.1 \mathrm{M}$ MES pH 6.5, 13\% PEG 400) and found that the activity of the enzymes was 10 times slower. Later, I adjusted the amount of the proteases such that all enzymes would digest similar amount of Xpo4 after certain time. Subsequently, I mixed different proteases with the elF5A ${ }^{15-154}$ export complex solution and set crystallization plates at $20{ }^{\circ} \mathrm{C}$. After 10 days, diamond shape crystals appeared in trypsin and chymotrypsin containing export complexes in $0.1 \mathrm{M}$ MES pH 6.26, 10-12\% PEG 400 (Figure 3-11). These crystals diffracted around 4-5 $\AA$ (the best diffraction was at $3.8 \AA$ ). The crystals belonged to trigonal space group $P 3_{1} 21$ (or its enantiomorph $P 3_{2} 21$ ) with unit cell dimensions $\mathrm{a}=\mathrm{b}=95.9 \AA, \mathrm{c}=379 \AA$ and contained one complex per asymmetric unit. 

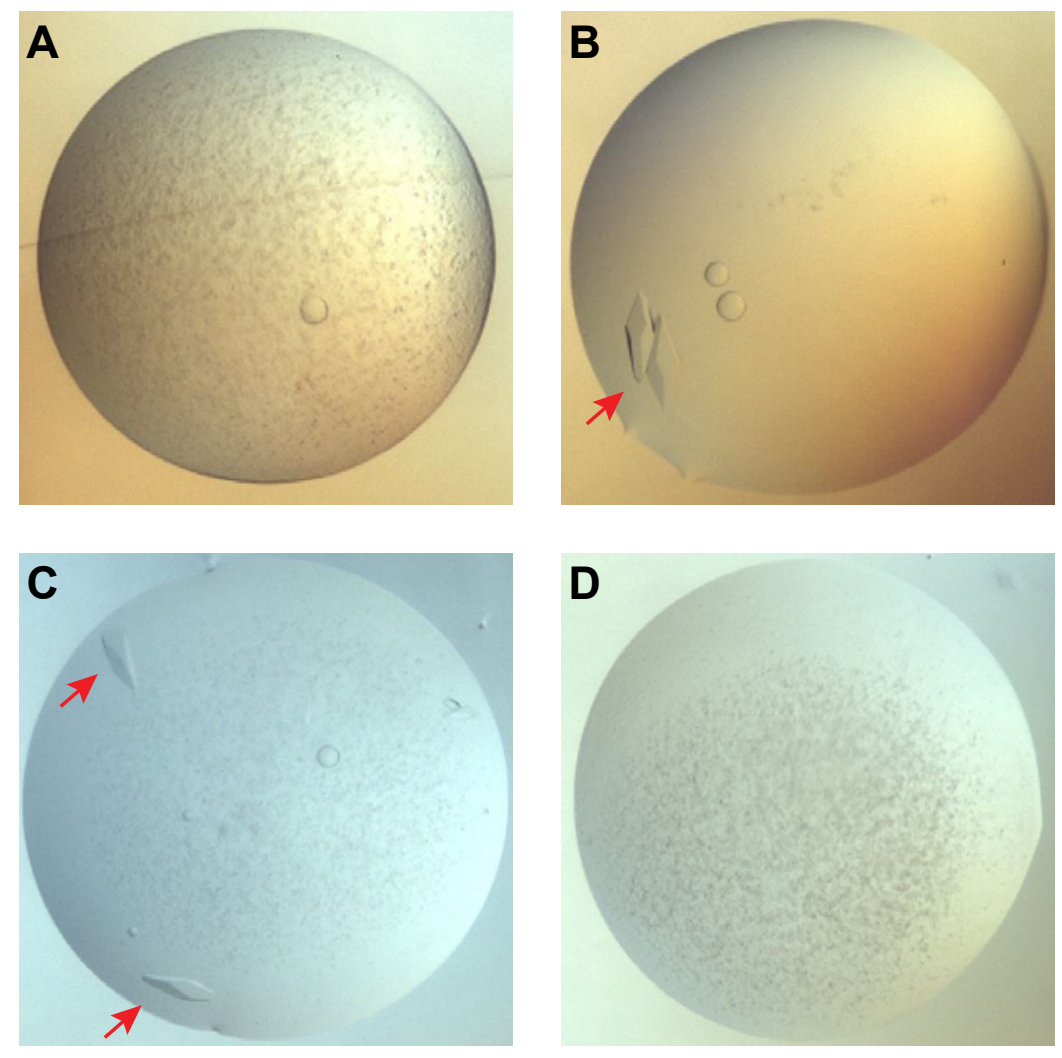

Figure 3-11 Crystallization drops of in situ limited proteolysis

$1 \mu \mathrm{L}$ of either the complex buffer, trypsin $(5.26 \mu \mathrm{g} / \mathrm{mL})$, chymotrypsin $(52.6 \mu \mathrm{g} / \mathrm{mL})$ or gluC $(100 \mu \mathrm{g} / \mathrm{mL}) \mathrm{was}$ mixed with $11 \mu \mathrm{L}$ of the export complex $(12 \mathrm{mg} / \mathrm{mL})$ and crystallization plates were set. Images of buffer $(\mathbf{A})$, trypsin (B), chymotrypsin (C) and gluC (D) containing drops of $0.1 \mathrm{M} \mathrm{MES} \mathrm{pH} \mathrm{6.26,} \mathrm{10.75 \%} \mathrm{PEG} 400$ are shown. The images were taken after 10 days.

Interestingly, the trypsin-digested crystals diffracted better than the chymotrypsin digested ones (approximately $1 \AA$ ). In order to check the content of the crystals, I picked single - trypsin- or chymotrypsin-digested — crystals and analyzed them by SDS-PAGE (Figure 3-12). The results had two important indications. First, the crystals were not homogenous. There were several Xpo4-like fragments similar to the in vitro limited proteolysis experiment. The intensity of the bands indicated that Xpo4 was not completely digested. Rather, the crystals contained full-length as well as partially digested Xpo4. Second, RanGTP was missing in the chymotrypsin-treated crystals. The band intensities of the Ran and elF5A were similar and stoichiometric in the trypsin-treated crystals. On the other hand, Ran was underrepresented in the chymotrypsin-treated one. This might imply the disruption of the complex by chymotrypsin treatment. 


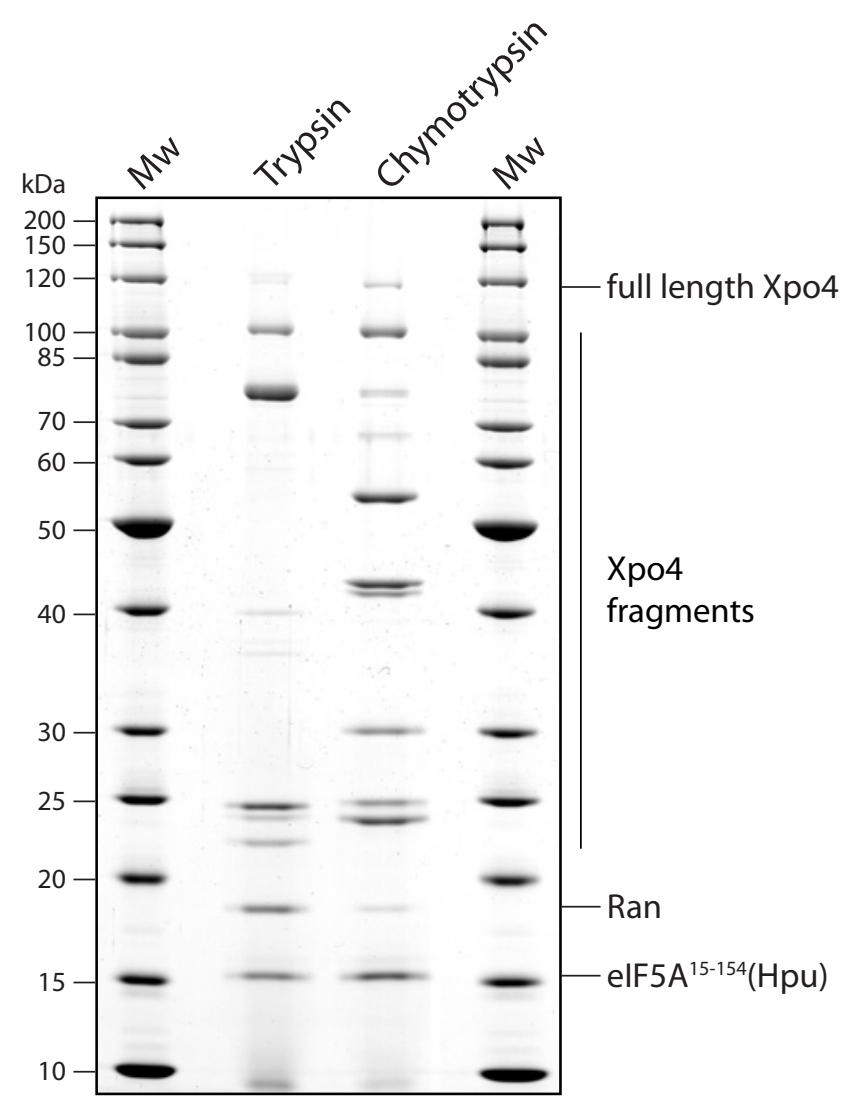

Figure 3-12 SDS-PAGE analysis of the trypsin and chymotrypsin digested crystals

Crystals obtained after the in situ limited proteolysis were fished out of the crystallization drop using Nylon loops, resuspended in SDS sample buffer and analyzed by SDS-PAGE followed by Coomassie blue staining. 'Mw' stands for molecular weight marker and protein sizes are marked on the left.

The loss of RanGTP and the heterogeneity of the crystals led us to reveal the fate of the complex after the protease digestions. I performed an in vitro limited proteolysis experiment followed by size exclusion chromatography. First, elF5A ${ }^{15-154}$ export complex was digested with trypsin or chymotrypsin at room temperature for $90 \mathrm{~min}$. Then, the digestions were subjected to a Superdex 200 10/30 gel filtration column. Finally, fractions of the peaks were analyzed by SDSPAGE. Figure 3-13 and Figure 3-14 demonstrate the elution profiles of the chromatography and the results of the SDS-PAGE analysis of trypsin and chymotrypsin digestions, respectively. 


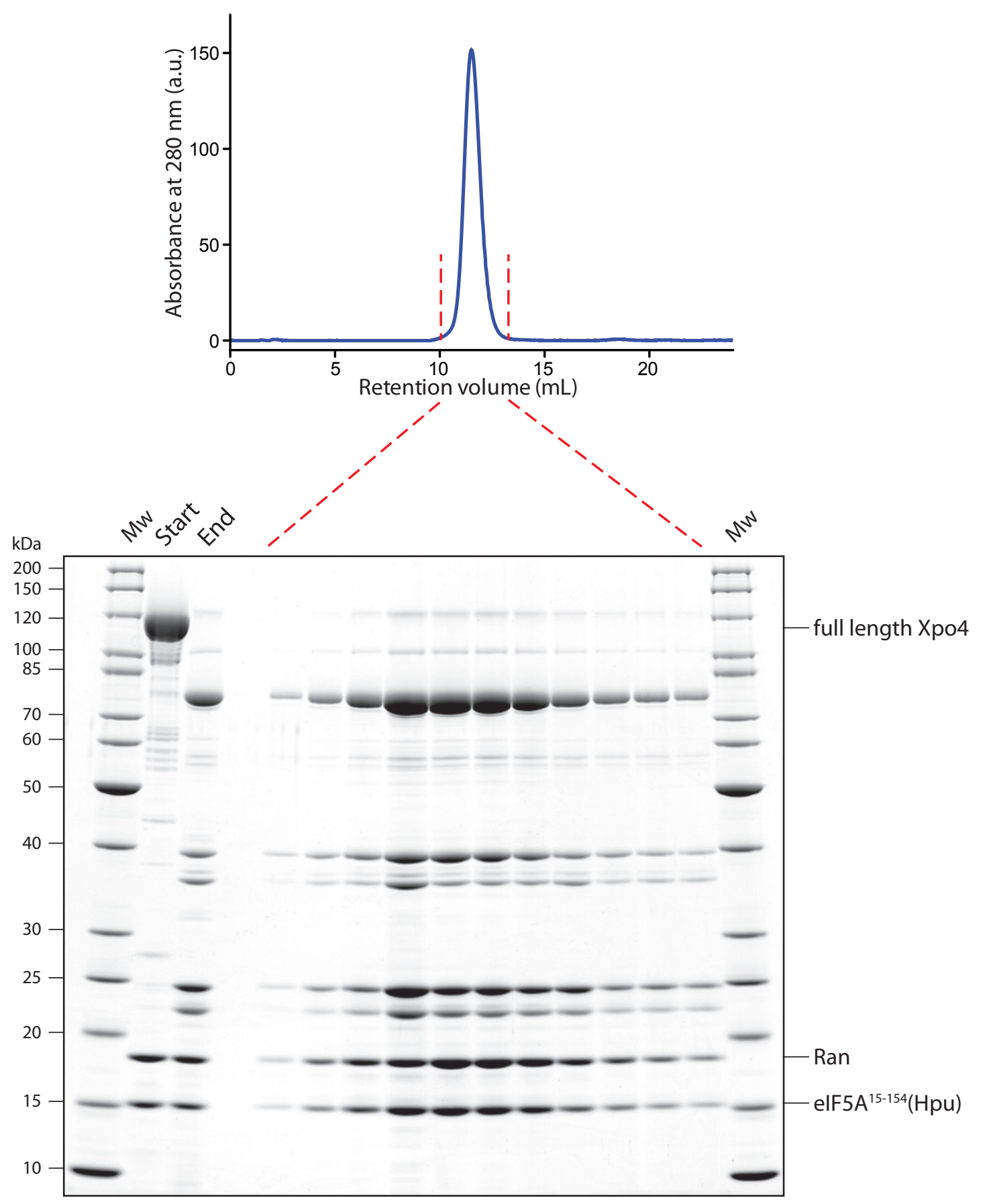

Figure 3-13 in vitro limited proteolysis with trypsin coupled to size exclusion chromatography

Limited proteolysis experiment was performed with trypsin and elF5A ${ }^{15-154}$ export complex (1:500 w/w trypsin to complex ratio) for $90 \mathrm{~min}$ at room temperature. The digestion was analyzed on a Superdex 200 10/30 gel filtration column equilibrated with $15 \mathrm{mM}$ Tris/ $\mathrm{HCl} \mathrm{pH} \mathrm{7.7,} 18 \mathrm{mM} \mathrm{NaCl}, 2 \mathrm{mM} \mathrm{Mg}(\mathrm{OAc})_{2}$ and $2 \mathrm{mM}$ DTT (upper panel). Eluted proteins were collected in $200 \mu \mathrm{L}$ fractions, precipitated with $10 \%$ TCA and resuspended in $25 \mu \mathrm{L}$ SDS sample buffer (supplemented with $10 \mathrm{mM}$ Tris base). $2 \mu \mathrm{g}$ of the export complex immediately after the protease addition (Start) and after 90 min incubation (End) and $10 \mu \mathrm{L}$ of the resuspended fractions were analyzed by SDS-PAGE followed by Coomassie Blue staining (lower panel). 'Mw' stands for molecular weight marker and protein sizes are marked on the left. (Modified from Aksu et al., 2016) 

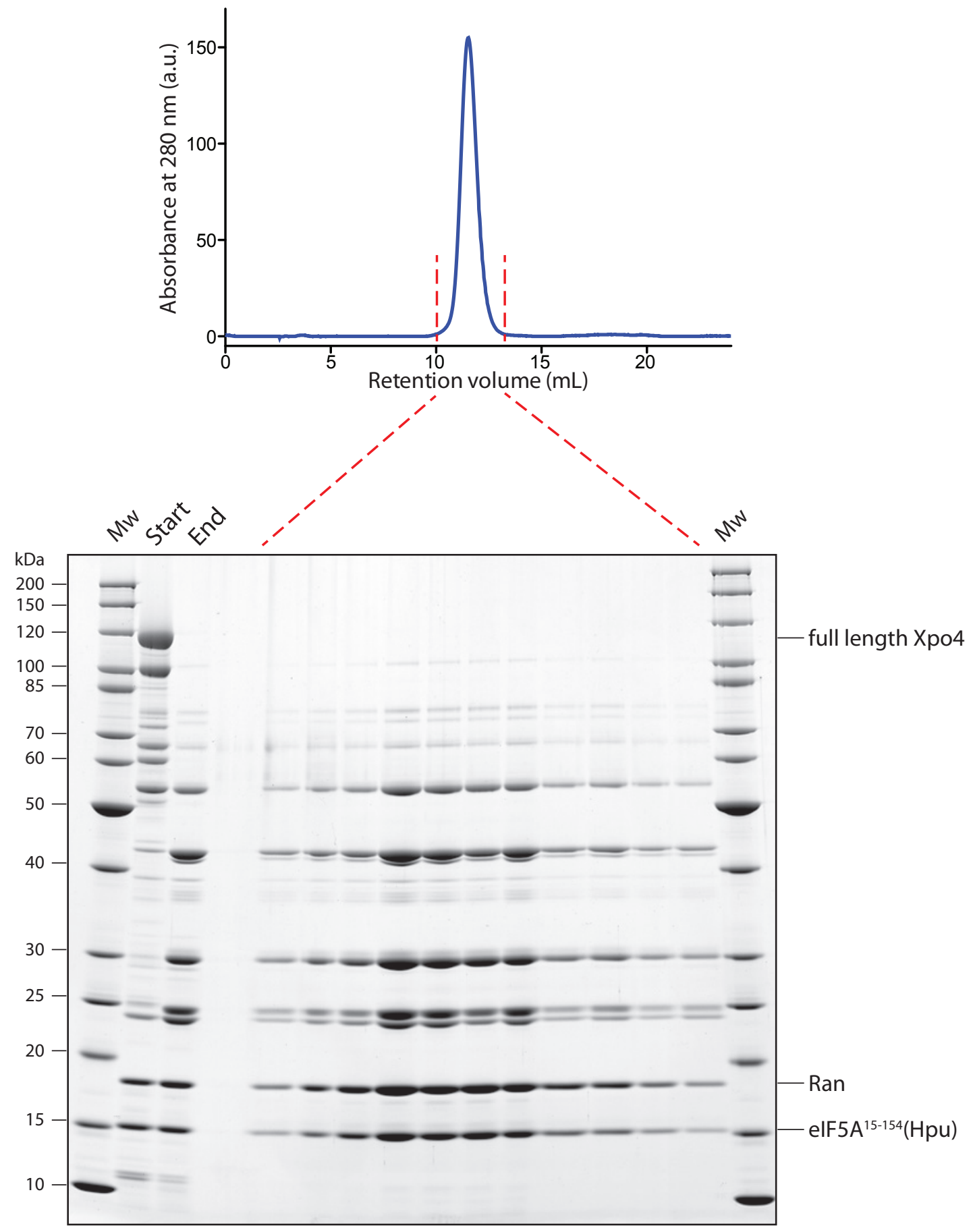

Figure 3-14 in vitro limited proteolysis with chymotrypsin coupled to size exclusion chromatography

Limited proteolysis experiment was performed with chymotrypsin and elF5A $\mathrm{A}^{15-154}$ export complex (1:100 w/w chymotrypsin to complex ratio) for $90 \mathrm{~min}$ at room temperature. The digestion was analyzed on a Superdex 200 10/30 gel filtration column equilibrated with $15 \mathrm{mM}$ Tris/ $\mathrm{HCl} \mathrm{pH} \mathrm{7.7,} 18 \mathrm{mM} \mathrm{NaCl}, 2 \mathrm{mM} \mathrm{Mg}(\mathrm{OAc})_{2}$ and $2 \mathrm{mM}$ DTT (upper panel). Eluted proteins were collected in $200 \mu \mathrm{L}$ fractions, precipitated with $10 \%$ TCA and resuspended in $25 \mu \mathrm{L}$ SDS sample buffer (supplemented with $10 \mathrm{mM}$ Tris base). $2 \mu \mathrm{g}$ of the export complex immediately after the protease addition (Start) and after $90 \mathrm{~min}$ incubation (End) and $10 \mu \mathrm{L}$ of the resuspended fractions were analyzed by SDS-PAGE followed by Coomassie Blue staining (lower panel). 'Mw' stands for molecular weight marker and protein sizes are marked on the left. 
Both of the digestions were eluted as a single peak and at the same elution volume of the native complex. SDS-PAGE analysis showed that although Xpo4 was cleaved at several positions by both of the proteases, all the fragments copurified and the digestions did not immediately disrupt the export complex. The results provided evidence that the proteases nicked the solvent-exposed loops of Xpo4 without affecting the stability of the complex.

Since the products of the in situ limited proteolysis were heterogeneous, I wanted to test if the relatively homogenous preparations would improve the quality of the crystals. I carried out preparative scale in vitro limited proteolysis using trypsin and chymotrypsin, subjected the digestions to size exclusion chromatography and set crystallization drops with the previously identified conditions. Remarkably, the digested complexes did not form crystals. In spite of all the efforts, I couldn't obtain any diffraction quality crystal. At best, some conditions gave needle like clusters. These results suggested that the in vitro limited proteolysis might have removed some of the additional loops of Xpo4 that might necessary for crystal contacts.

\section{Generation of loop deletions and crystallization}

So far, we were able to obtain diffracting quality crystals with the export complex, however, couldn't improve the resolution of the crystals. The limited proteolysis experiments and crystallization trials suggested that the removal of certain regions of Xpo4 was crucial for crystallization whereas the removal of other regions prevented crystallization. The heterogeneity of the Xpo4 in the crystals seemed to be the primary reason for relatively poor resolution. It may have been challenging to find out the conditions that would have homogenous Xpo4 digestions as well as give rise to well diffracting crystals. Therefore, we decided to create an engineered Xpo4 that lacks the solvent-exposed loops and test if the complex (containing loop deleted Xpo4) would form crystals in the absence of proteases.

This task had two important questions to be resolved: What were the exact positions of the loops and how many of these loops had to be removed for crystal formation? To answer these questions, I made use of bioinformatics tools and combined the obtained information with biochemical assays. 

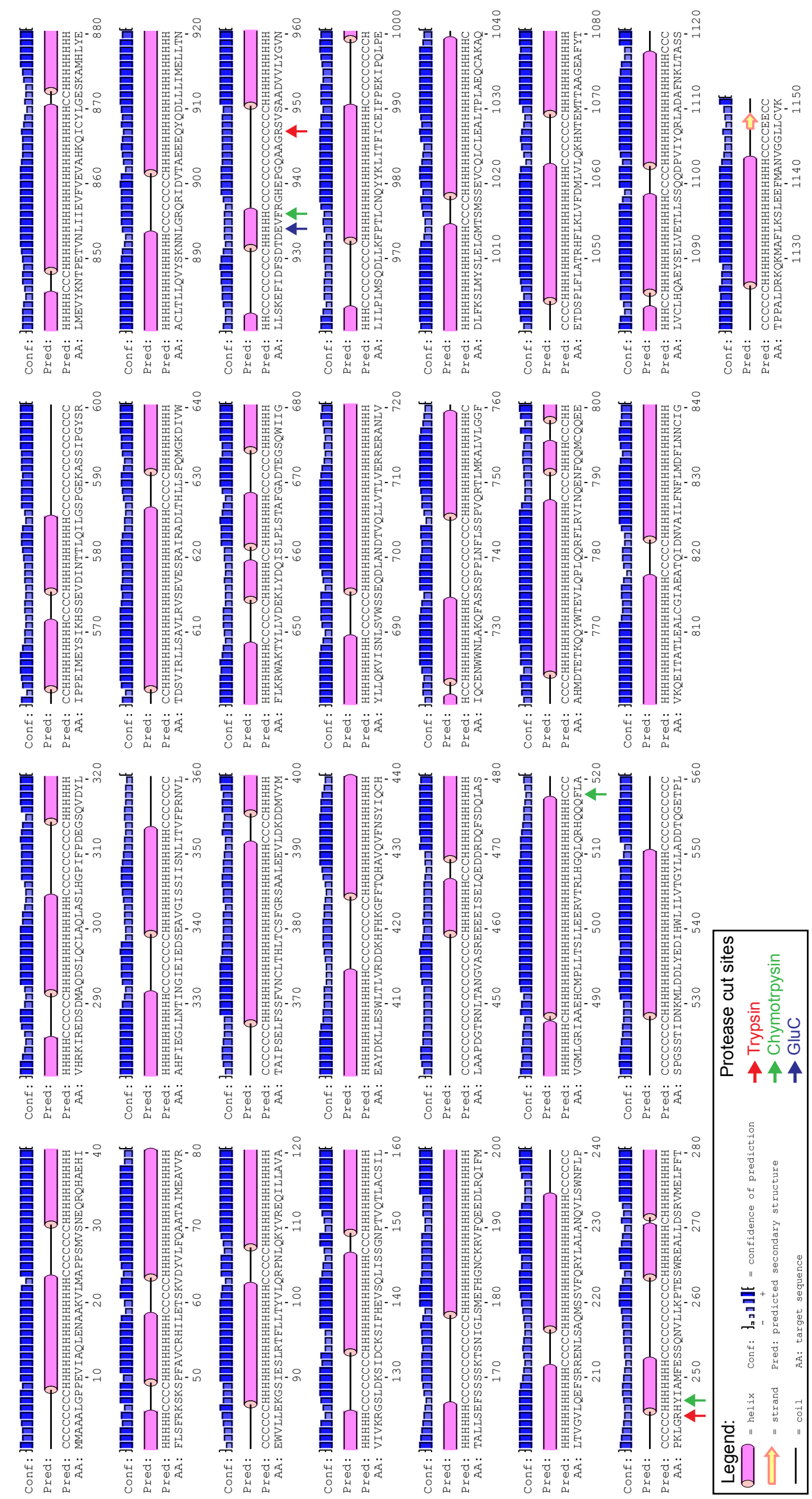

Figure 3-15 Secondary structure prediction of Xpo4 


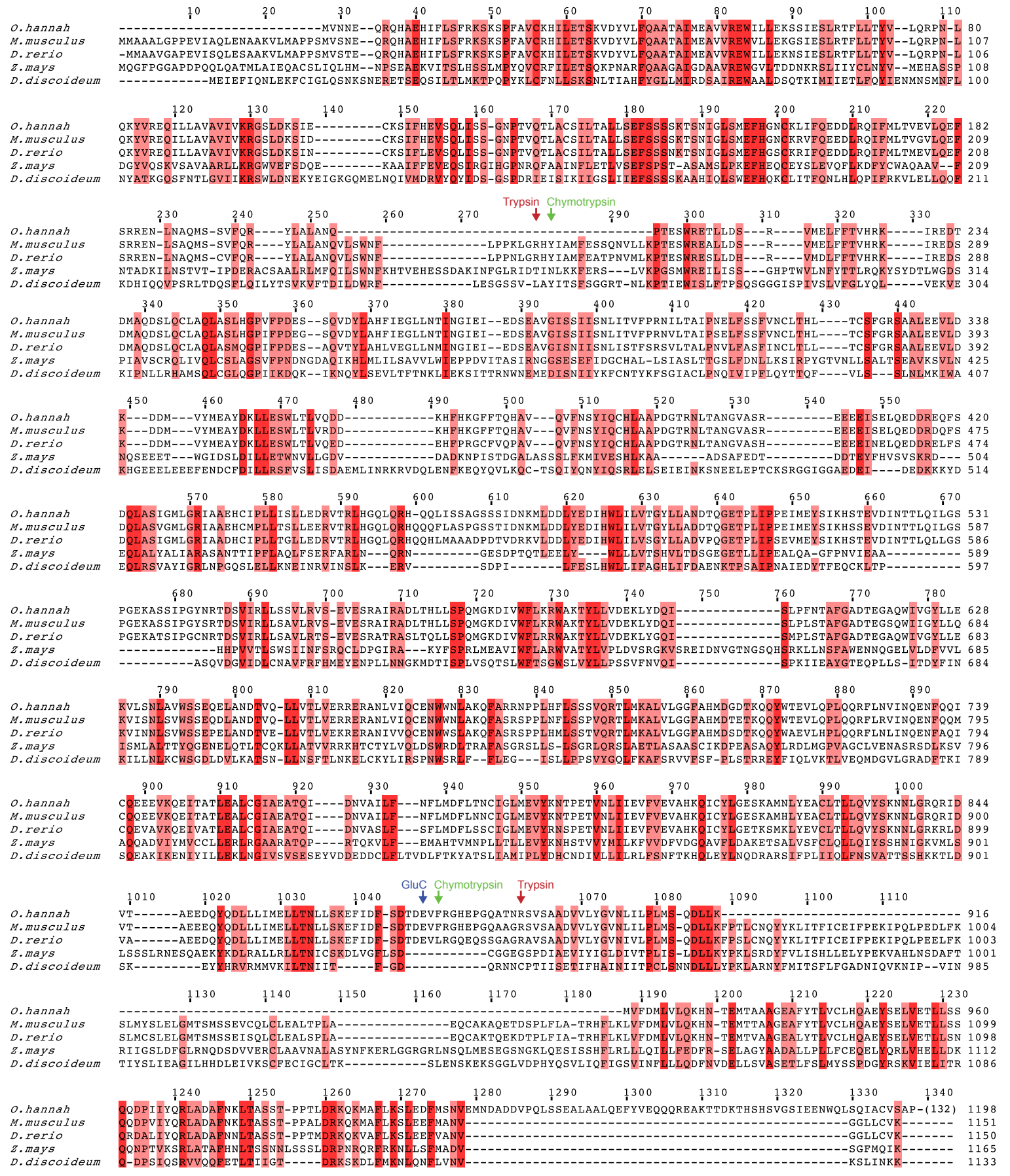

Figure 3-16 Sequence alignment of Xpo4 homologues

The alignment includes Xpo4 from M. musculus (mouse), D. rerio (zebrafish), O. Hannah (king cobra), $Z$. mays (maize), and $D$. discoideum. The conserved residues are highlighted in dark red boxes, whereas the residues that are present in four out of five sequences are in light red boxes. Identified protease cleavage sites are marked with arrows and indicated accordingly. (Modified from Aksu et al., 2016)

I used online secondary structure prediction algorithms to find out the predicted positions of the HEAT repeats. One example from PSIPRED server is shown in Figure 3-15. In parallel, I examined the conservation of Xpo4 homologues from different organisms. Figure 3-16 shows the sequence alignment of Xpo4 homologues from five different organisms. 
Consistent with the flexible loops, the residues surrounding the protease cleavage sites (residues 240-260 and 930-950) are poorly conserved among Xpo4 homologues and even missing in some organisms (Figure 3-16). Moreover, these regions reside in long loops, or in helices with low confidence value, which are surrounded by helices with high confidence value (possibly the helices of the HEAT repeats) (Figure 3-15).

I wanted to remove as many amino acids as possible without disturbing the structure of Xpo4. In order to determine the number of residues that can be deleted from Xpo4, I made use of the following approach. Based on the secondary structure prediction, I set the borders and therefore the maximum size of the loops that can be deleted. Later, I constructed several Xpo4 deletion mutants lacking different size of loops. Next, assuming that the solubility of a protein reflects the proper folding, I compared the solubility of these mutants with full-length Xpo4. Finally, I checked the ability of these mutants to bind RanGTP and elF5A, picked the best and combined different loop deletions. Accordingly, I identified the loop at the $\mathrm{N}$-terminus to be the residues between 241 and 260, and the loop at the C-terminus to be the residues between 931 and 948; and created the mutants depicted in Figure 3-17.

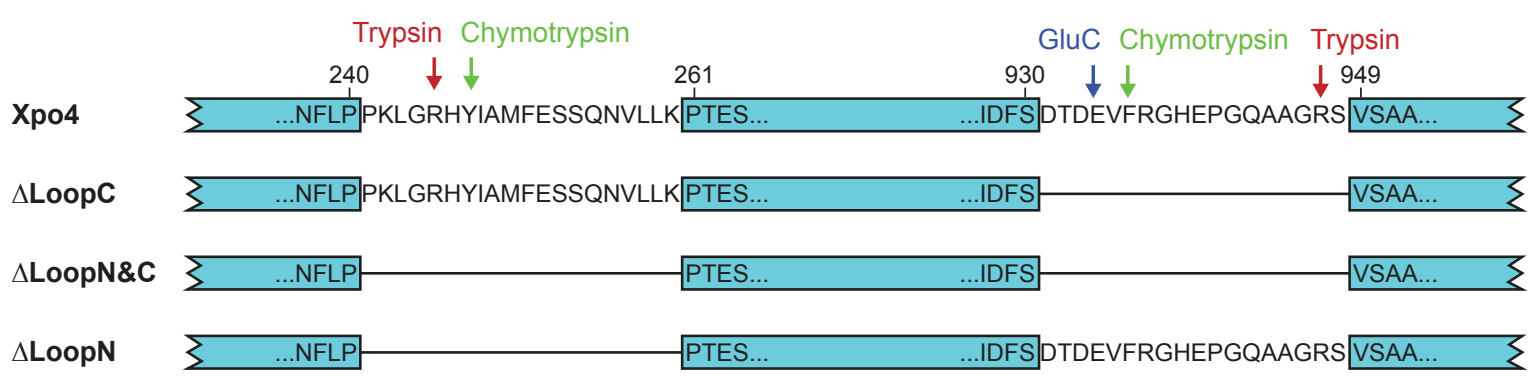

Figure 3-17 Schematic representation of the Xpo4 loop mutants

The sequences of the characterized loops are shown in Xpo4 (the full-length). The protease cleavage sites are marked with arrows and shown above the corresponding sequence. $\Delta$ LoopC, $\Delta$ LoopN\&C and $\Delta$ LoopN represent Xpo4 ${ }^{\Delta 931-948}, \mathrm{Xpo}^{\Delta 241-2608931-948}, \mathrm{Xpo}^{\Delta 241-266}$, respectively. (Adapted from Aksu et al., 2016)

I formed elF5A export complexes with these mutants using the protocol established for the full-length Xpo4, analyzed them by size exclusion chromatography and SDS-PAGE (Figure 3-18). All complexes eluted nearly at the same volume and contained all the components of the export complex. SDSPAGE analysis showed that all the mutants formed stoichiometric complexes with RanGTP and elF5A(Hpu). Later, I subjected these complexes to limited 
proteolysis with trypsin. Confirming our previous biochemical data, $\Delta$ LoopN\&C mutant was resistant to proteolytic degradation at the $\mathrm{N}$ - and $\mathrm{C}$-terminal regions (Figure 3-18b).
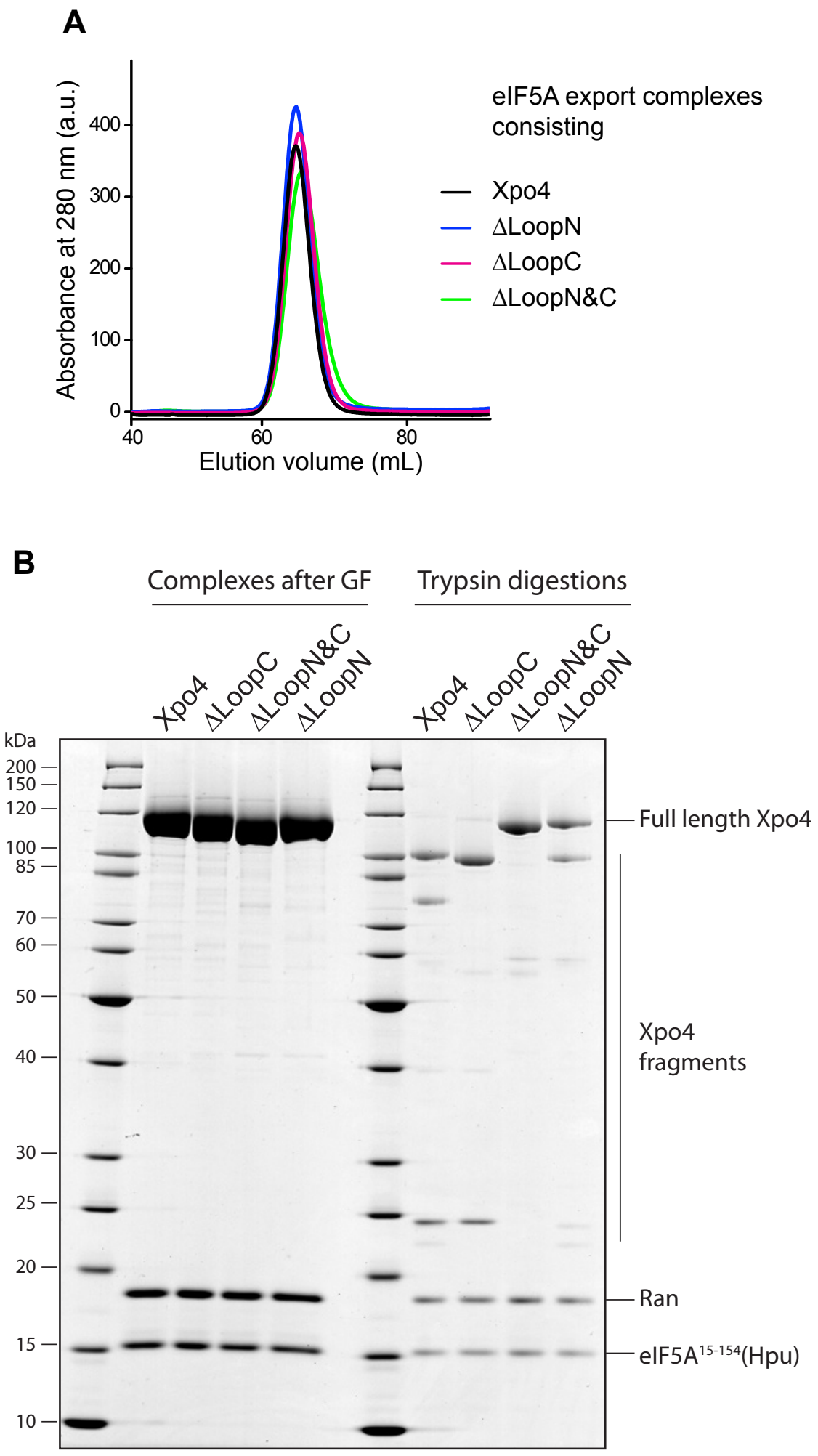

Figure 3-18 elF5A export complex formation with Xpo4 loop mutants

(A) Overlay of the size exclusion chromatograms of the export complexes consisting Xpo4 or Xpo4 loop mutants. Export complexes were formed in solution using near stoichiometric amounts of elF5A(Hpu), 
RanGTP and Xpo4 or Xpo4 mutants. Each complex was analyzed on a Superdex $20016 / 60$ gel filtration column equilibrated with $15 \mathrm{mM}$ Tris/ $\mathrm{HCl} \mathrm{pH} \mathrm{7.7,} 18 \mathrm{mM} \mathrm{NaCl}, 2 \mathrm{mM} \mathrm{Mg}(\mathrm{OAc})_{2}$ and $2 \mathrm{mM}$ DTT. (B) Left Peak fractions were collected, pooled and concentrated to $12 \mathrm{mg} / \mathrm{ml} .2 \mu \mathrm{g}$ from each complex was analyzed by SDS-PAGE followed by Coomassie staining. Right The export complexes were incubated with trypsin (1000:1 $\mathrm{w} / \mathrm{w})$ for $1 \mathrm{~h}$ at $22^{\circ} \mathrm{C}$ and digestion pattern ( $1 \mu \mathrm{g}$ from each complex) was analyzed by SDS-PAGE followed by Coomassie staining. (Adapted from Aksu et al., 2016)

I set crystallization drops with these complexes using the previously identified crystallization conditions. Export complexes consisting of either $\triangle \mathrm{LoopN}$ or $\triangle$ LoopN\&C were crystallized without any protease treatment (Figure 3-19). In addition, in situ trypsin- or chymotrypsin-treated $\Delta$ LoopC containing export complex also formed similar crystals with the same crystallization condition. Overall, these results suggested that the removal of N-terminal loop of Xpo4 was required for proper crystal contacts. Remarkably, crystals obtained from the full-length Xpo4 containing complex could be used as seeds for both $\triangle$ LoopN and $\triangle$ LoopN\&C complexes suggesting that new crystals had similar unit cell properties as the full-length.
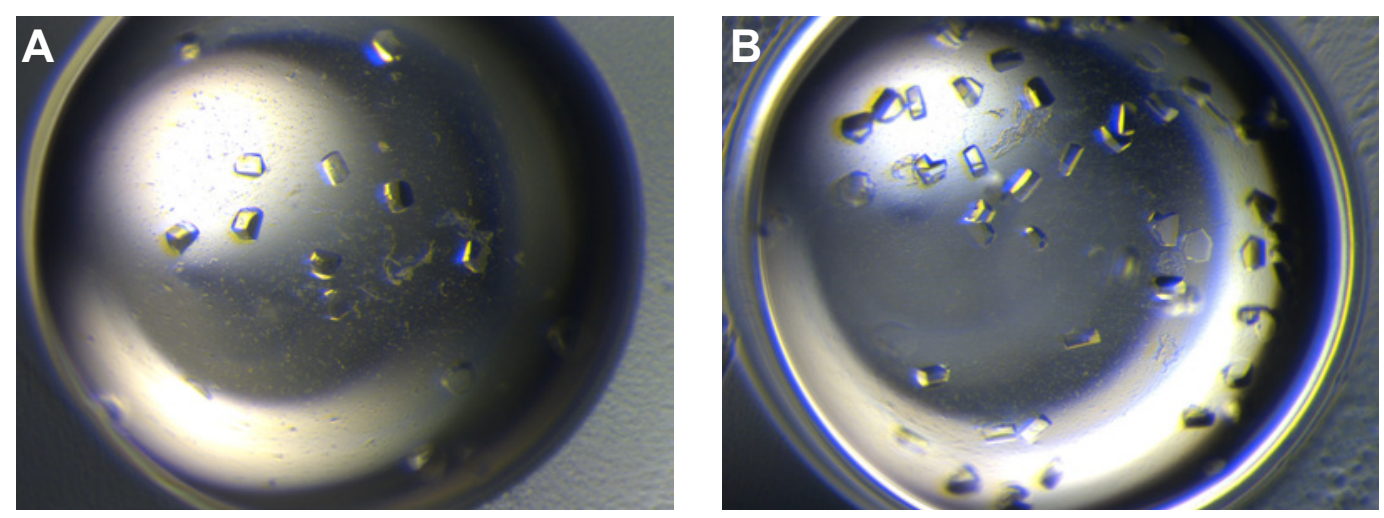

Figure 3-19 Crystallization of the export complexes consisting Xpo4 loop mutants

$1 \mu \mathrm{L}$ of $12 \mathrm{mg} / \mathrm{ml}$ export complex was mixed with $1 \mu \mathrm{L}$ of the precipitants with various $\mathrm{pH}$ and PEG concentrations. Export complexes consisting either $\Delta$ LoopN $(\mathbf{A})$ or $\Delta$ LoopN\&C (B) were crystallized in $0.1 \mathrm{M}$ MES pH 6.26, 8-10\% PEG 400. Note that the crystallization condition is almost identical to that of in situ protease treated full-length Xpo4 containing export complexes.

These crystals diffracted to around $3.5 \AA$. We tested several crystals and collected a data set at $3 \AA$ resolution from the $\triangle$ LoopN\&C containing export complex. The crystals belonged to trigonal space group $P 3_{1} 21$ with unit cell dimensions $\mathrm{a}=\mathrm{b}=$ 98.6 $\AA$ and $\mathrm{c}=726.8 \AA$ and contained two complexes per asymmetric unit. Interestingly, the size of the unit cell in one dimension (c) was double compared to the old crystals, possibly to accommodate the second complex in the asymmetric unit. For phasing, I purified selenomethionine-labeled $\Delta$ LoopN\&C, formed ternary export complex, crystallized with the same crystallization condition and collected a data set at $3.5 \AA$ resolution, where the anomalous signal extended beyond $5 \AA$ resolution. 


\subsubsection{Structure of elF5A export complex}

\section{Structure determination}

All data collection was done at beamline X10SA at the Swiss Light Source (Villigen, Switzerland) together with Dr. Sergei Trakhanov from our lab. High redundancy of the collected data was necessary for phase determination. For phasing information, the dataset was indexed, integrated and scaled with XDS. Positions of the 56 selenium sites (out of 66) were located by SHELXD. Both the steps were carried out by Dr. Trakhanov. Later, I obtained the initial phases by molecular replacement (MR) using RanGTP (PDB ID 3GJX; Monecke et al., 2009) as the search model. The resulting information and the position of selenium atoms were used to obtain the electron density map. Initial helix and strand search revealed the ring like structure of Xpo4 as well as the position of Ran. Remaining electron density at this point clearly demonstrated the position of elF5A. Crystal structure of human elF5A (PDB ID 3CPF; Tong et al., 2009) was later manually placed to the electron density. Automated model building was performed using AutoBuild Wizard in Phenix. Later, Coot was used for manual model building and PHENIX Refine was used for refinement. The quality of the final model was assessed by MolProbity as well as by the validation server of PDB. In the final stages, the model was refined against the data set at a resolution of $3.2 \AA$ to an Rwork of $23.3 \%$ and Rfree of $29.9 \%$. The model has good stereochemistry, with $96.4 \%$ of the residues in the most favored region of the Ramachandran plot and only two residues in the disallowed region. Data collection and refinement statistics are shown in Table 3-3 and a sample of the quality of the electron density for the interaction interfaces is provided in Figure 3-20. The crystal structure contains two ternary complexes in the asymmetric unit, which are very similar (r.m.s.d. of $1 \AA$ over 904 atom pairs). Complex 1 has better electron density than complex 2, therefore I will refer to complex 1 unless otherwise stated. The final model includes residues 7 to 176 of Ran and 16 to 151 of elF5A. I modeled 1025 of 1113 residues of Xpo4, missing few residues at the very $\mathrm{N}$ - and C-termini as well as several disordered loop regions between the HEAT repeats. Due to weak electron density at the $\mathrm{C}$ terminus of Xpo4, last two HEAT repeats were modeled mostly as polyalanine. 


\begin{tabular}{|c|c|c|}
\hline & Native & SeMet \\
\hline \multicolumn{3}{|l|}{ Data collection } \\
\hline Space group & \multicolumn{2}{|c|}{$P 3,21$} \\
\hline \multicolumn{3}{|l|}{ Cell dimensions } \\
\hline$a, b, c(\AA)$ & $98.616,98.616,726.864$ & $98.477,98.477,725,678$ \\
\hline$\alpha, \beta, \gamma\left({ }^{\circ}\right)$ & $90,90,120$ & $90,90,120$ \\
\hline Resolution $(\AA ̊)$ & $49.41-3.2(3.31-3.20)^{*}$ & $49.33-3.40(3.61-3.48)$ \\
\hline$R_{\text {sym }}$ & $0.08(0.95)$ & $0.15(1.96)$ \\
\hline $\mid / \sigma l$ & $22.42(2.26)$ & $18.0(1.40)$ \\
\hline Completeness (\%) & 99.91 (99.79) & $99.7(97.10)$ \\
\hline Redundancy & $9.90(10.20)$ & $18.7(15.10)$ \\
\hline \multicolumn{3}{|l|}{ Refinement } \\
\hline Resolution $(\AA)$ & $49.41-3.20$ & \\
\hline No. reflections & 69829 & \\
\hline$R_{\text {work }} / R_{\text {free }}(\%)$ & 23.3/29.9 & \\
\hline \multicolumn{3}{|l|}{ No. atoms } \\
\hline Protein & 18644 & \\
\hline Ligand/ion & 66 & \\
\hline Water & 0 & \\
\hline \multicolumn{3}{|l|}{$B$-factors } \\
\hline Protein & 102.7 & \\
\hline Ligand/ion & 84.1 & \\
\hline \multicolumn{3}{|l|}{ Water } \\
\hline \multicolumn{3}{|l|}{ R.m.s deviations } \\
\hline Bond lengths $(\AA)$ & 0.006 & \\
\hline Bond angles $\left(^{\circ}\right)$ & 0.81 & \\
\hline
\end{tabular}

Table 3-3 Data collection and refinement statistics for RanGTP.Xpo4-elF5A complex

*Values in parentheses are for highest-resolution shell 
A
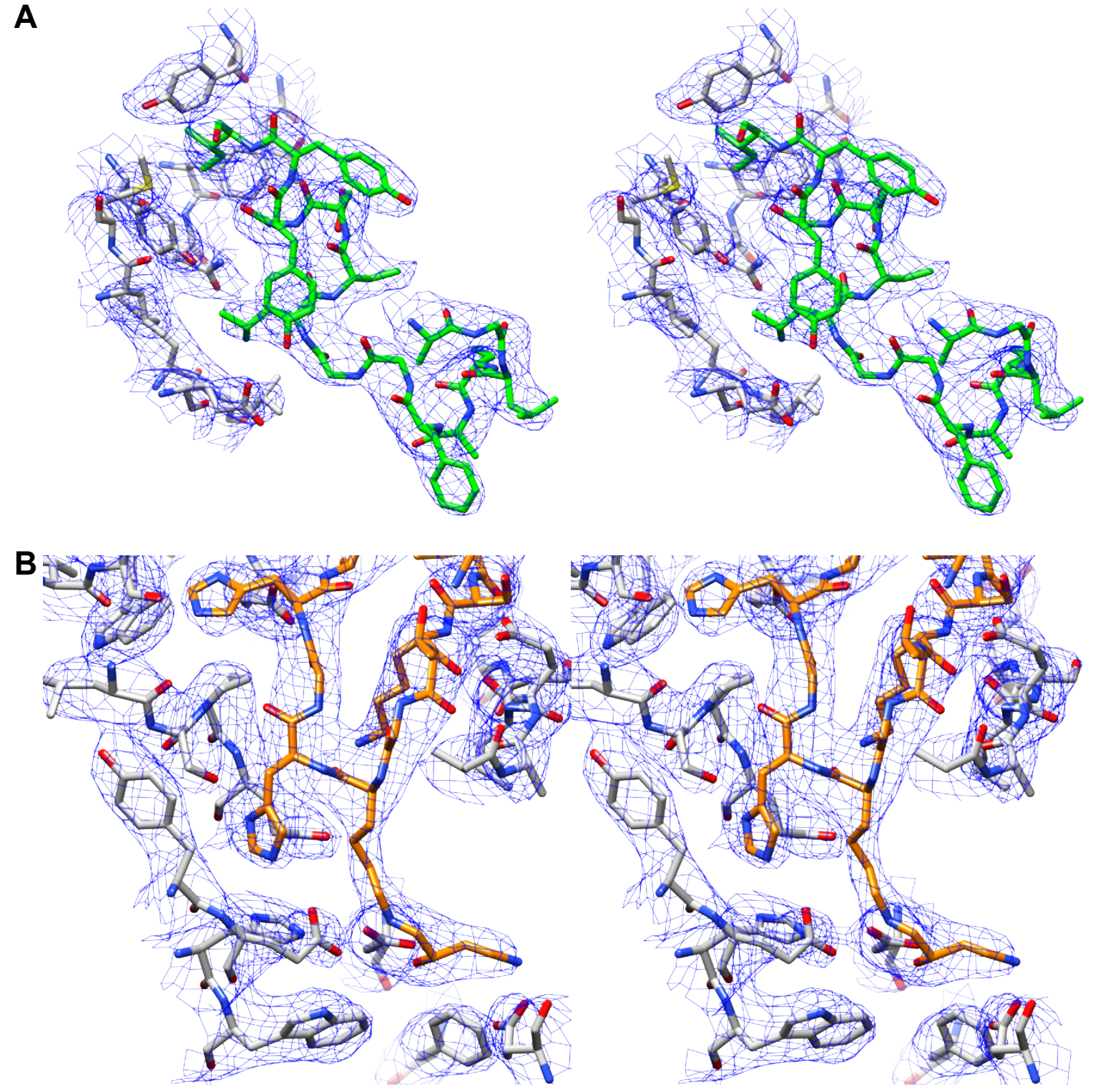

Figure 3-20 Stereo views of the electron density of the export complex structure

The electron density of the refined 2Fo-Fc map (contoured at $1.0 \mathrm{\sigma}$ ) is shown as blue mesh, with the stick representation of the final model superimposed. (A) Stereo view of the switch II region of Ran (carbon atoms in green) interacting with Xpo4 (carbon atoms in grey). (B) Stereo view showing the hypusine-containing loop of elF5A (carbon atoms in orange) docking into Xpo4's acidic pocket. (Adapted from Aksu et al., 2016) 


\section{Overall structure of Xpo4}

The structure of the export complex is shown in Figure 3-21. Before explaining the details of the export complex structure, I would like to devote this section to describing the structure of Xpo4.
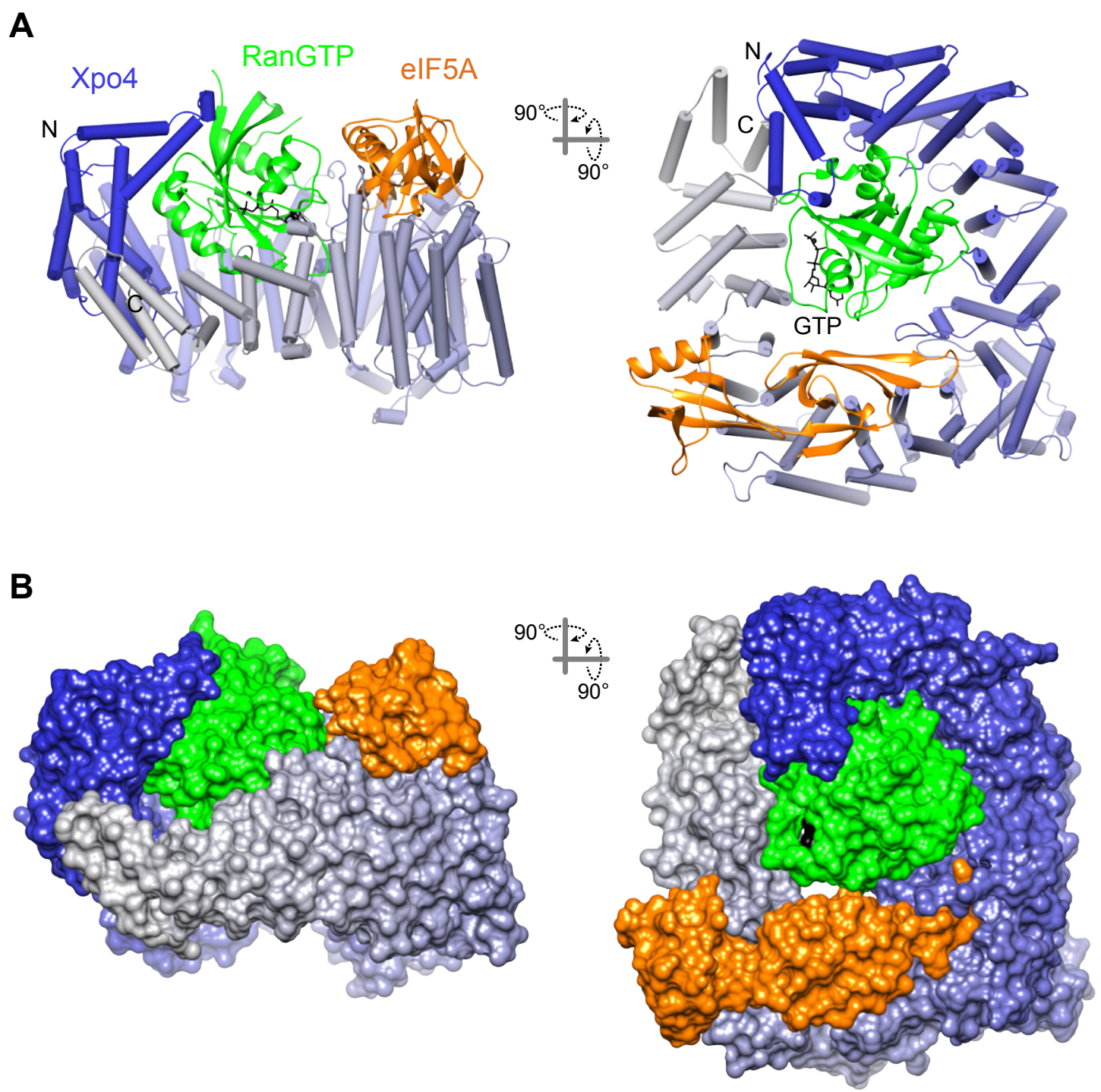

Figure 3-21 Structure of the RanGTP·Xpo4-elF5A export complex

(A) View of the export complex in two different orientations. Ran (green) and elF5A (orange) are shown in a ribbon representation, whereas Xpo4 is shown as cylinders. GTP (black) is shown as sticks. Xpo4 is depicted with a color gradient from blue ( $\mathrm{N}$ terminus) to grey ( $\mathrm{C}$ terminus). (B) The export complex is rendered as surface representation with same color-coding and view as in $(\mathbf{A})$. 

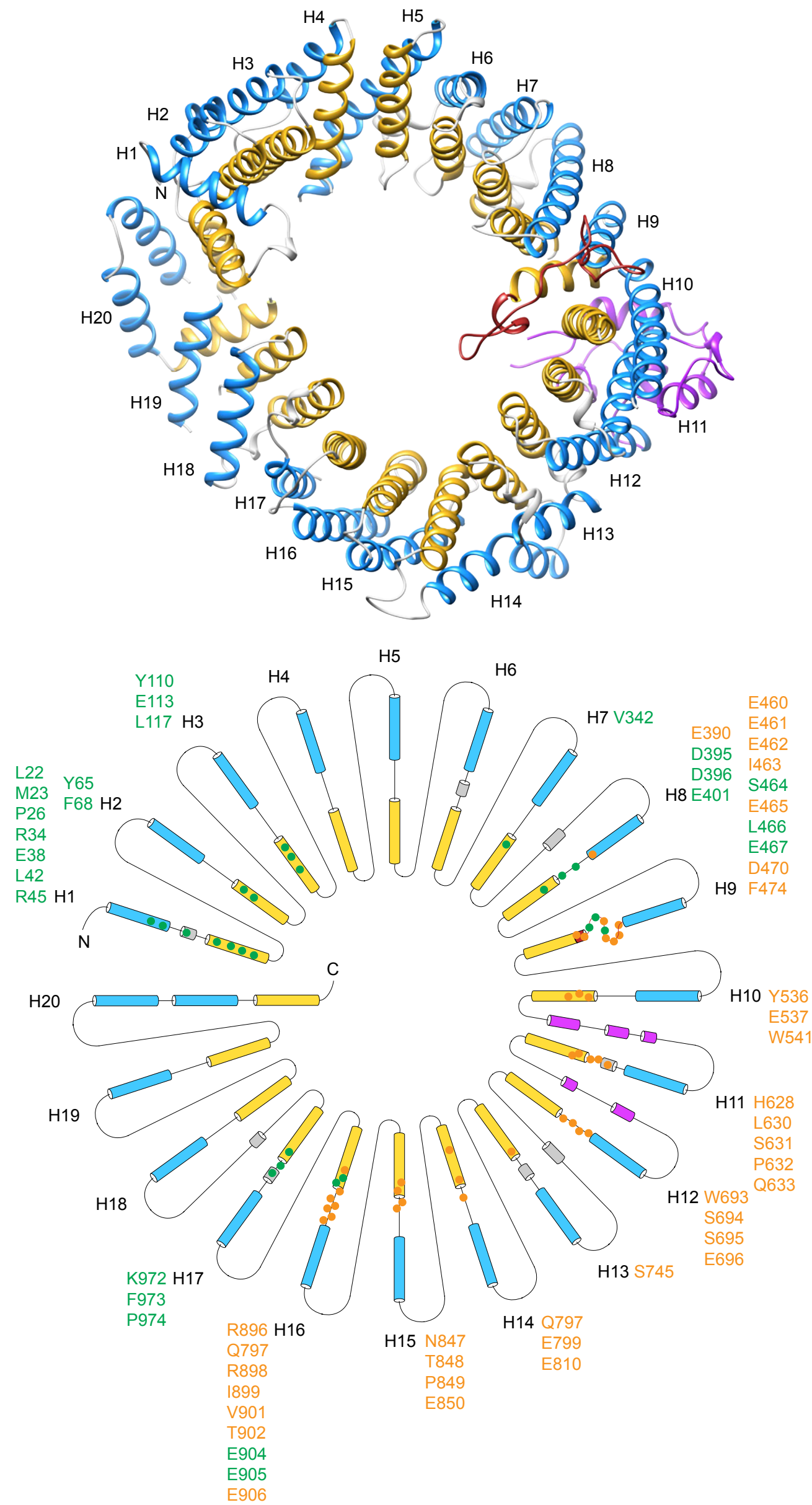
Upper. Xpo4 in the export complex is shown in a ribbon representation (RanGTP and elF5A are removed for clarity). A and B helices of the HEAT repeats are colored in blue and yellow, respectively. Long inter-repeat insertions mentioned in the text are colored in dark pink, whereas the acidic loop is in brown. Bottom. Schematic representation of the Xpo4 secondary structure. Coloring is same as in the upper panel. Green and orange dots represent the Xpo4 residues interacting with RanGTP and elF5A, respectively. (Adapted from Aksu et al., 2016)

Xpo4, as all members of importin $\beta$ family, is an all $\alpha$-helical protein and is made of consecutive HEAT repeats (Figure 3-22). As mentioned in the introduction, HEAT repeats are $\sim 40$ amino acid motifs which consist of two consecutive $\alpha$-helices (A and $B$ ) that pack in an antiparallel orientation against each other (Andrade et al., 2001). The repeats pack side by side to form a superhelical structure. The $A$ helices form the outer convex surface and the $B$ helices form the inner convex surface (Figure 3-22). Xpo4 consists of 19 canonical HEAT repeats and 3 ahelices (termed as HEAT 20) at the very C-terminus sealing the superhelix. Superhelical arrangement of Xpo4 is interrupted by three anticlockwise kinks (between HEATs 3 and 4, HEATs 9 and 10, and HEATs 13 and 14) that convert superhelical structure into a toroid-like shape, with HEAT 20 touching the loop between HEATs 2 and 3.

Xpo4 contains several insertions either between the $A$ and $B$ helices of the same HEAT repeat (intra-repeat) or between successive HEAT repeats (inter-repeat). Most of the intra-repeat insertions are short loops and in some cases contain small helices. An exception is the $~ 30$ amino acid long loop in between HEAT 9A and 9B. This loop contains mostly acidic residues and resembles the so-called 'acidic loop' of CRM1 (Monecke et al., 2009). The inter-repeat insertions, on the other hand, vary in length and topology. Noteworthy insertions are between HEATs 10 and 11 and HEATs 11 and 12. The insertion between HEATs 10 and 11 is 50 amino acid long and folds into two a-helices, one of them packing against the $A$ helices of HEATs 10 and 11. Similarly, the insertion between HEATs 11 and 12 is a long loop ( 30 amino acids) with a hydrophobic a helix folding against the $\alpha$ helices of the previous insert. 


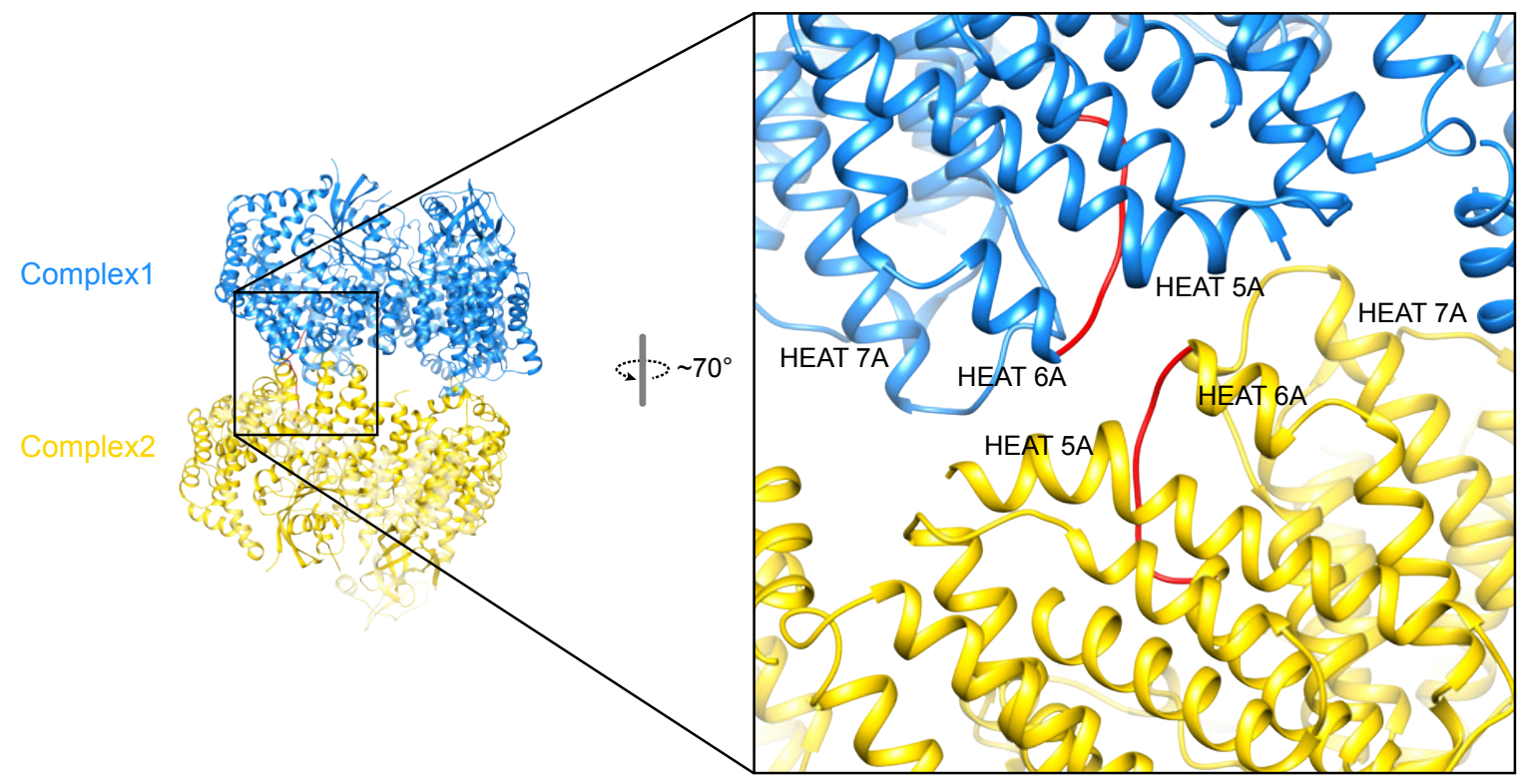

Figure 3-23 Complexes at the asymmetric unit and Xpo4 contact sites

On the left, the components of the asymmetric unit are shown in ribbon representation. Ternary complexes are colored in blue and yellow. On the right, the magnified view shows the portions of Xpo4 that interact with each other around the deleted N-terminal loop (red). In general, HEATs 5-7 Xpo4 from complex1 are in close proximity to the same region of Xpo4 from complex 2. Additional 20 residues into the loop shown in red would prevent the proper contact, validating our initial work.

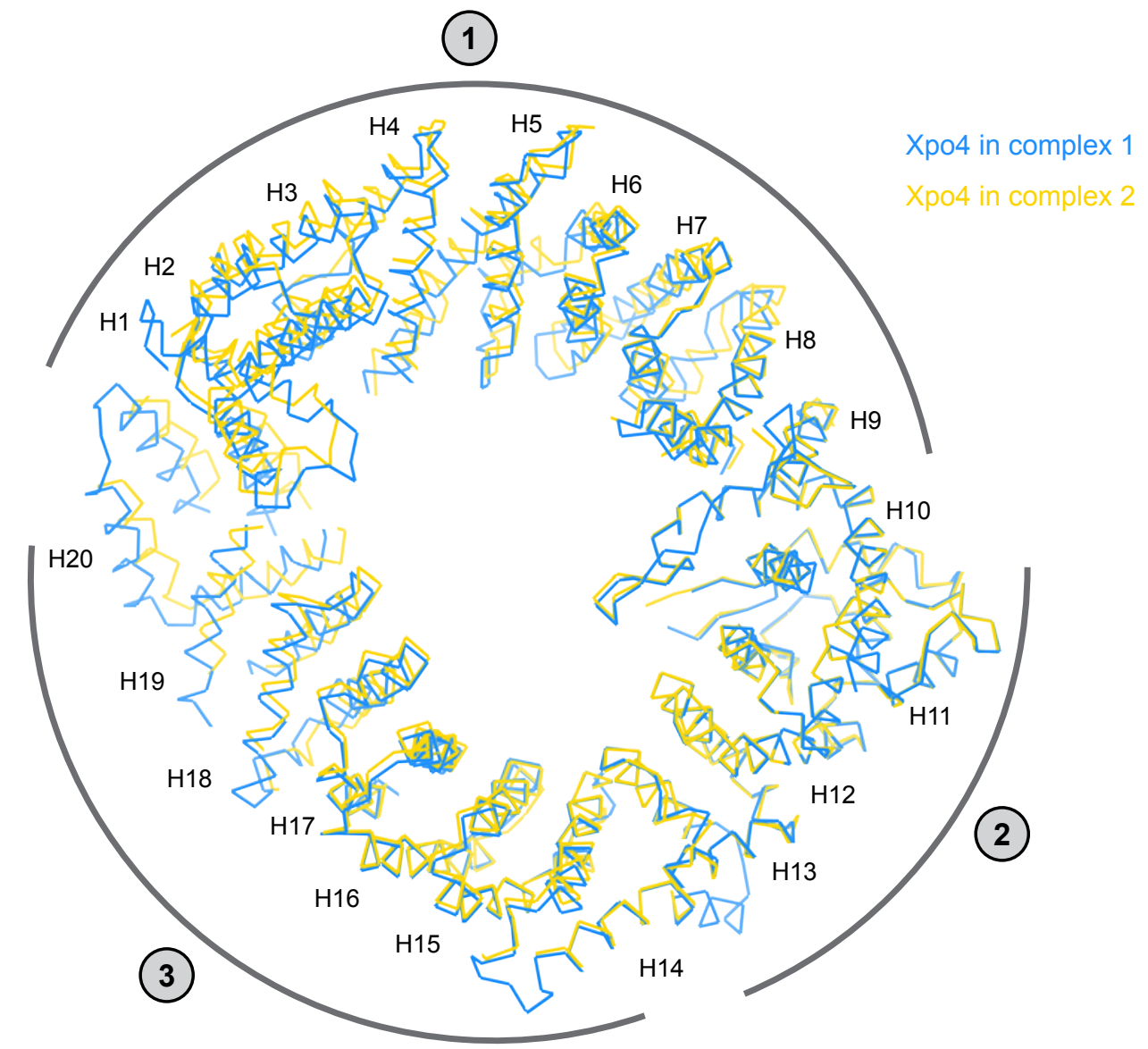

Figure 3-24 Conformational differences between the Xpo4 molecules in the asymmetric unit

Xpo4 from complex 1 (blue) is superimposed to that from complex 2 (yellow) via HEATs 10-13. Conformational differences between the Xpo4 molecules indicate possible hinge regions for the movement of $\mathrm{N}$ - and C-terminal HEAT repeats, which divide Xpo4 into 3 rigid bodies: HEATs 1-9, 10-13, and 14-20. 
As previously identified, two regions of Xpo4 were sensitive to certain proteases and the deletion of the N-terminal loop was crucial to obtain crystals. The $\mathrm{N}$-terminal and $\mathrm{C}$-terminal deletions reside in the loops between HEATs 5 and 6 and HEATs 16 and 17, respectively. In the asymmetric unit, the residues in the loop between HEATs 5 and 6 contact the same residues of the second Xpo4 molecule, providing a rationale for the necessity of the deletion (or the protease treatment in the initial case) to obtain the diffraction quality crystals (Figure 3-23).

Two Xpo4 molecules in the asymmetric unit are identical and superimpose with an r.m.s.d of $1 \AA$ in their Ca atoms (Figure 3-24). However, the r.m.s.d is lower if superposition is carried out using only HEAT repeats 1-9 (0.5 $\AA$ ), HEAT repeats 10-13 (0.4 $\AA$ ) or HEAT repeats $14-20(0.7 \AA)$, indicating a slight movement of both termini with respect to each other. This might also reflect the flexibility of Xpo4.

\section{RanGTP recognition by Xpo4}

Xpo4 wraps around Ran and has four distinct interaction sites. The details are shown in Figure 3-25 and Figure 3-22 (lower panel). The first interaction site involves the $\mathrm{N}$-terminal region of Xpo4 and is formed by the HEATs 1 to 3 . This region interacts with switch II region of Ran (residues 65 to 80 ) as well as a helix 3. The interaction occurs mainly via hydrophobic contacts, which is very similar to what has been seen for other NTRs. In fact, N-terminal is the most conserved region among the importin $\beta$ superfamily (Görlich et al., 1997). HEATs 7 and 8 form the second interaction site and contact the basic back of Ran involving the so-called 'basic patch', $\beta$ strand 6 and $\alpha$ helix 5. In particular, Asp395, Asp396 and Glu401 of Xpo4 interact with His139, Arg140, Gln145, Trp163 and Arg166 of Ran. Overall this interaction resembles the interaction between Ran and the conserved acidic insertion of importin $\beta$ and transportin (Chook and Blobel, 1999; Vetter et al., 1999a). The third interaction site of Xpo4 is formed by the acidic loop within HEAT 9, which engages contacts with the loops of Ran involved in guanine recognition. A similar Ran-binding interface is found in most of the exportins. However, in CAS, Xpot and Xpo5, the interactions are mediated by the loops within the C-terminal HEAT repeats (Matsuura and Stewart, 2004; Cook et al., 2009; Okada et al., 2009). Therefore, this mode of Ran-binding is analogous to 
that of CRM1 as the interaction site is also formed by the acidic loop within HEAT 9 (Monecke et al., 2009). However, it is noteworthy to mention that unlike CRM1, which contacts Ran mostly via electrostatic interactions, interaction of Xpo4 occurs via hydrophobic contacts centered at Leu466 of Xpo4. The last Ran-binding interface involves the C-terminal repeats (HEATs 16 and 17), which contacts switch I of Ran (residues 30 to 47). Other exportins (except Xpo5) also contact switch I of Ran by the C-terminal HEAT repeats.

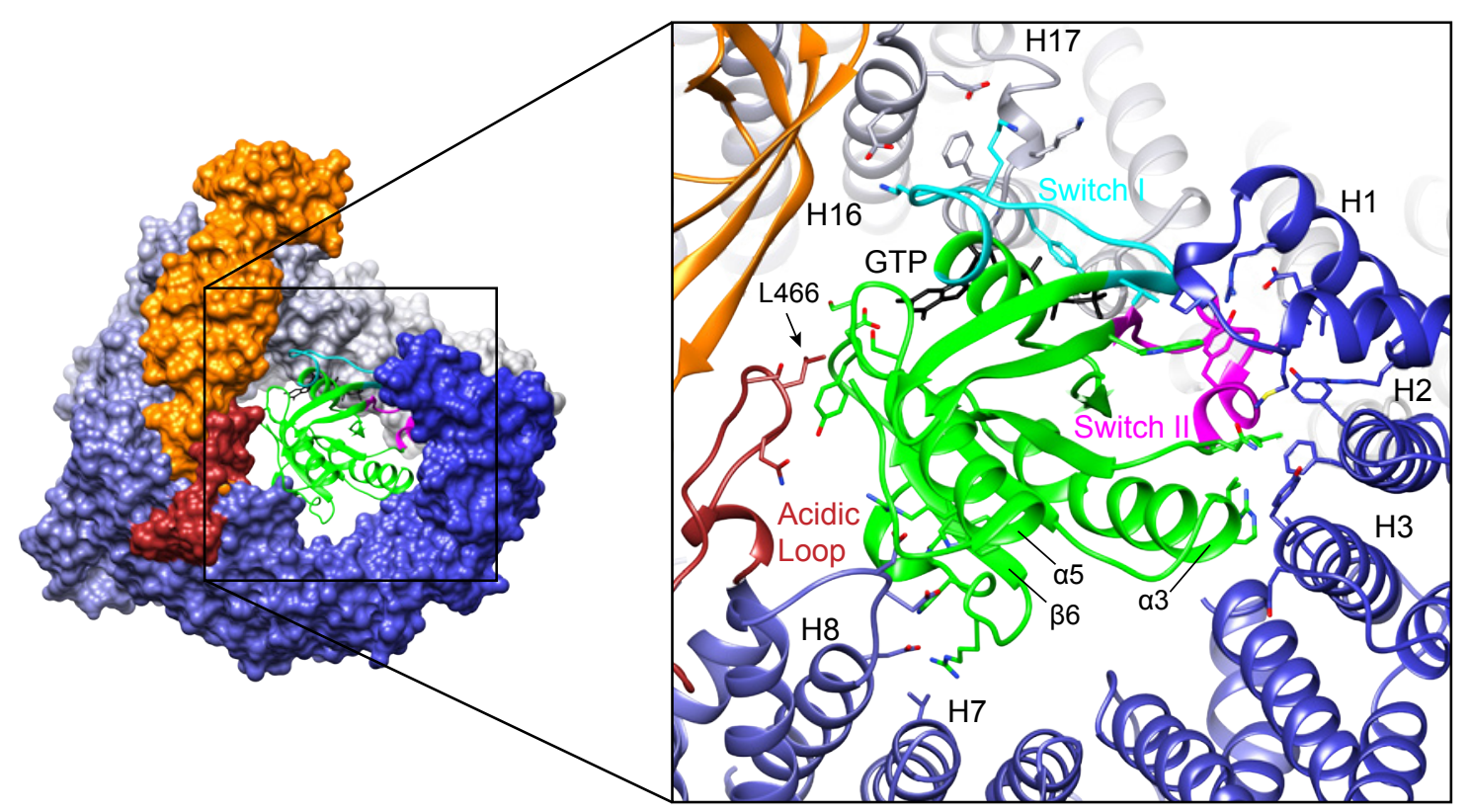

Figure 3-25 Recognition of RanGTP by Xpo4

On the left, Xpo4 and elF5A are rendered as surface representation. Xpo4 is shown with a color gradient from blue ( $\mathrm{N}$ terminus) to grey (C terminus), while acidic loop is shown in brown. elF5A is colored in orange and Ran in green. Switch I and II regions of Ran are shown as cyan and pink, respectively. GTP (black) is shown as sticks. On the right, the magnified view shows the interacting residues of Xpo4 and RanGTP. (Adapted from Aksu et al., 2016)

The structure of Ran in the export complex is almost identical (with an r.m.s.d of $0.5 \AA$ ) to that in the other NTR-RanGTP complexes. Although the details of the interactions of Xpo4 with Ran differ at certain regions, the overall recognition mechanism is similar to that seen in other exportins. Xpo4 contacts switch I and switch II of Ran, hence directly sensing its nucleotide-bound state. These regions have different conformations in GDP-bound Ran; therefore, the interactions described for these regions would not occur. Indeed, if RanGDP structure is overlaid with RanGTP in the export complex, switch I and II regions of RanGDP would clash with HEAT 1 and HEATs 1 to 3, respectively (Figure 3-26). Additionally, switch III region of RanGDP, which is disordered in GTP-bound form, 
would clash with HEAT 8 and the acidic loop of Xpo4. Overall, these conformational differences make RanGDP incompatible for Xpo4 binding.

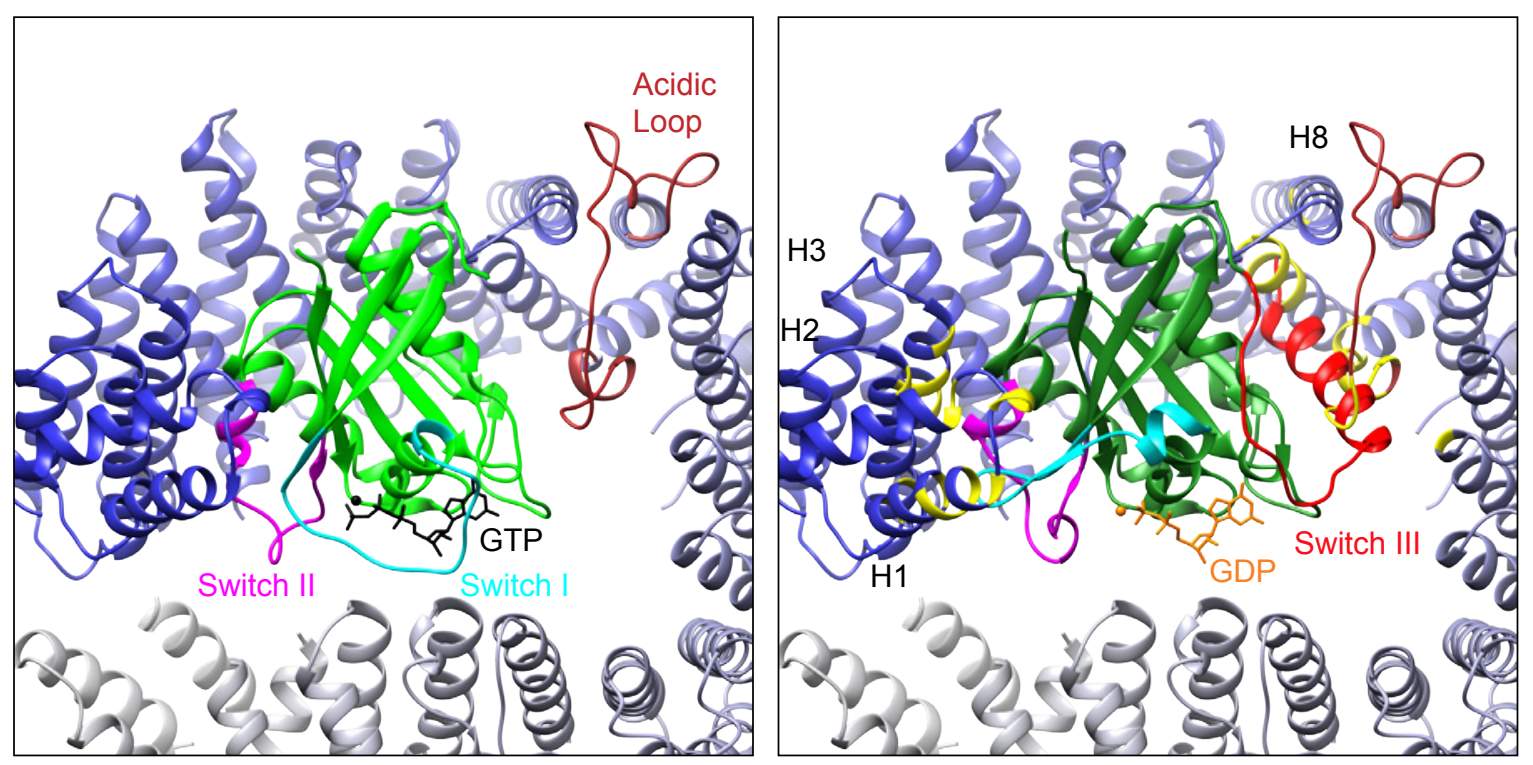

Figure 3-26 RanGDP is incompatible for Xpo4 binding

On the left, the structure of the export complex is shown (elF5A is removed). Structure components were colored as in Figure 3-25. On the right, structure of GDP-bound Ran (PDB ID 3GJ0, Partridge and Schwartz, 2009 ) is superimposed to the export complex. Ran is shown in dark green, switch III region in red and GDP in orange. Xpo4 residues that would clash with Ran are colored in yellow. 


\section{Interactions of elF5A with Xpo4}

The structure of unmodified human elF5A has been previously solved (Tong et al., 2009). elF5A consists of two domains, an N-terminal SH3-like domain and a Cterminal oligonucleotide-binding (OB)-fold domain, connected by a short linker (Figure 3-27). In the export complex, the structure of each domain is essentially similar (with an r.m.s.d of $0.6 \AA$ for each) to that in the previous structure. The only difference is a $15^{\circ}$ anticlockwise rotation of the $\mathrm{C}$-terminal domain relative to the $\mathrm{N}$-terminal domain, which is most probably enforced by the Xpo4 as it would otherwise clash. Consistent with our biochemical data, the very $\mathrm{N}$-terminus of elF5A is solvent-exposed (Figure 3-28b lower panel).
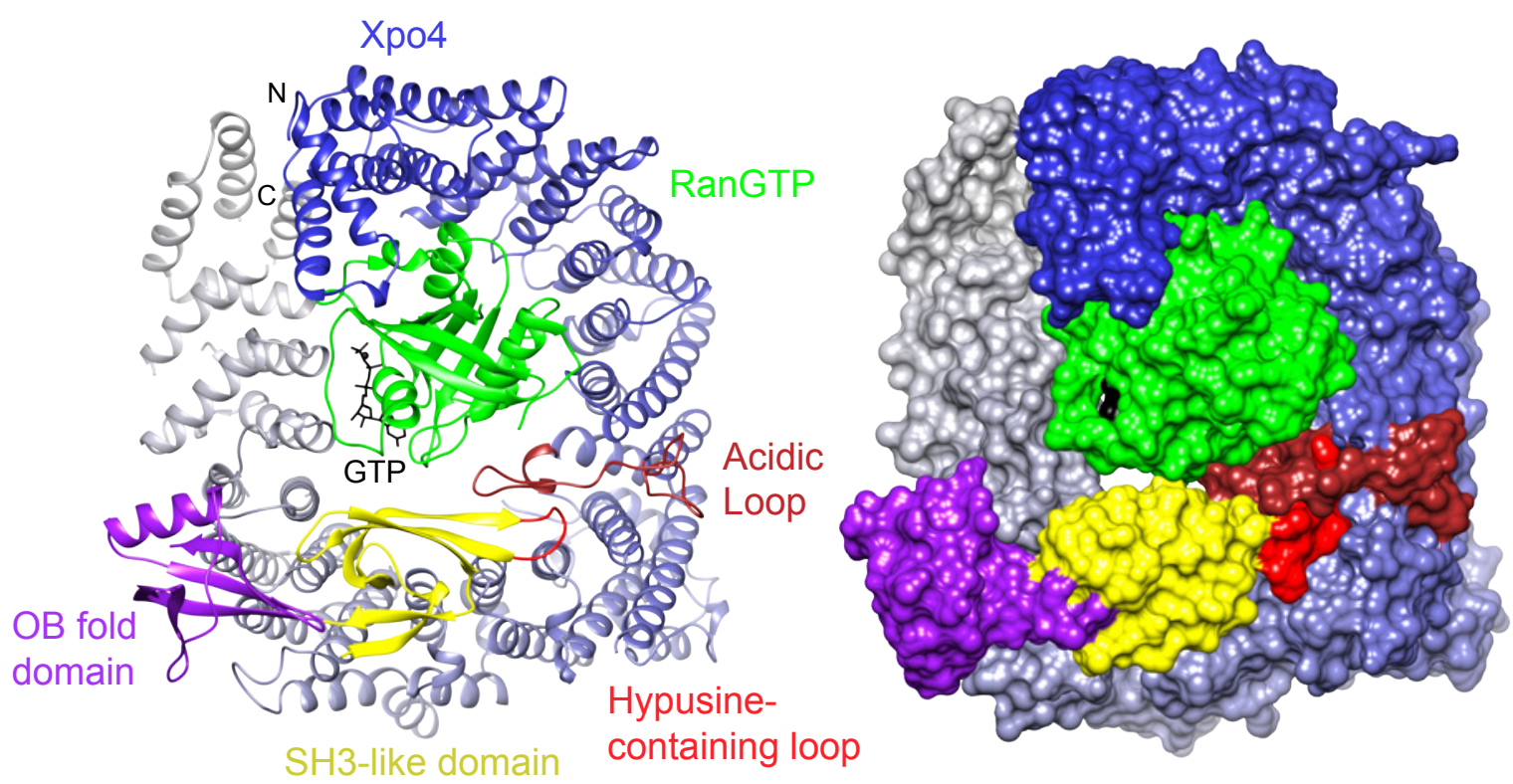

Figure 3-27 Structure of the export complex and domains of elF5A

The structure of the export complex is depicted as in Figure 3-21 (right panels), with the structural domains of elF5A colored and indicated accordingly.

Xpo4 interacts extensively with both domains of elF5A (Figure 3-27), burying a total surface of $2169 \AA^{2}$ on elF5A. This is consistent with the biochemical data that revealed contributions from both domains for Xpo4 binding (Lipowsky et al., 2000). Xpo4 does not wrap its cargo; instead elF5A sits on the intra-repeat loops of HEATs 11 to 16 , while inserting the basic hypusine-containing loop into an acidic pocket made up of HEATs 8 to 11 (Figure 3-27 and Figure 3-22 bottom panel). Several negatively charged residues of Xpo4 form the interaction interface, which are complemented with conserved positively charged residues of elF5A (Figure 3-28c). 
A

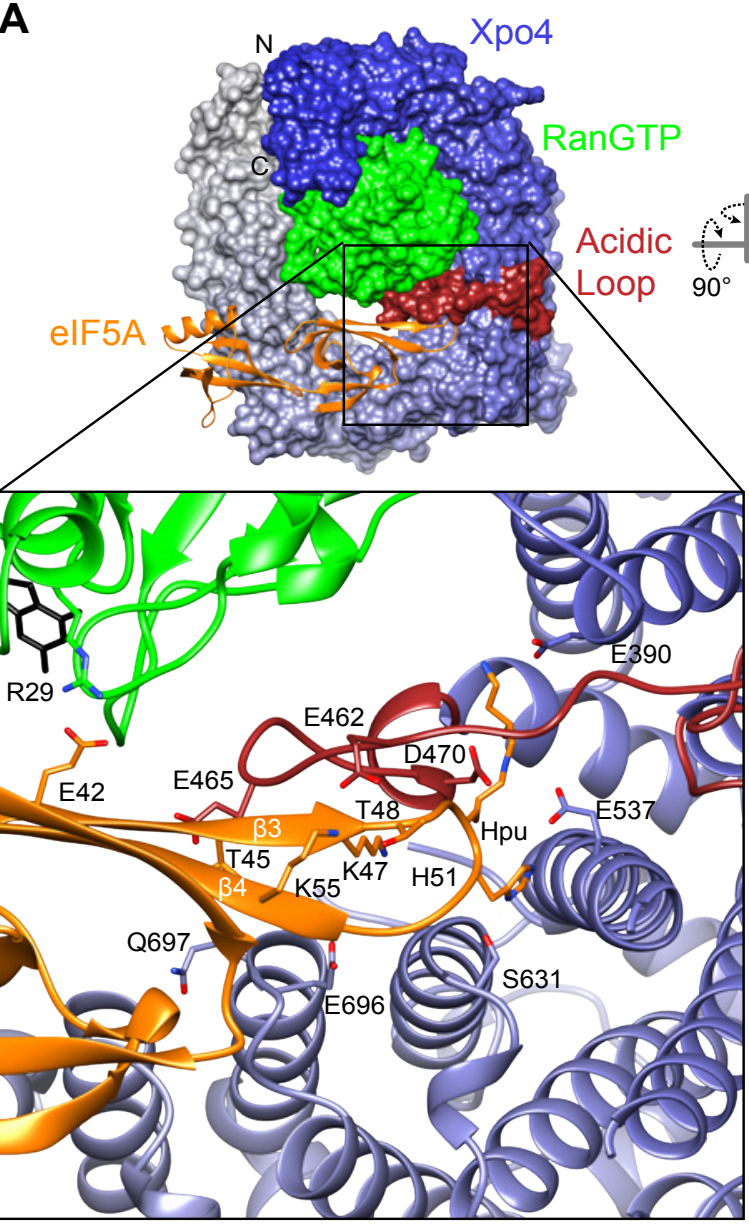

C

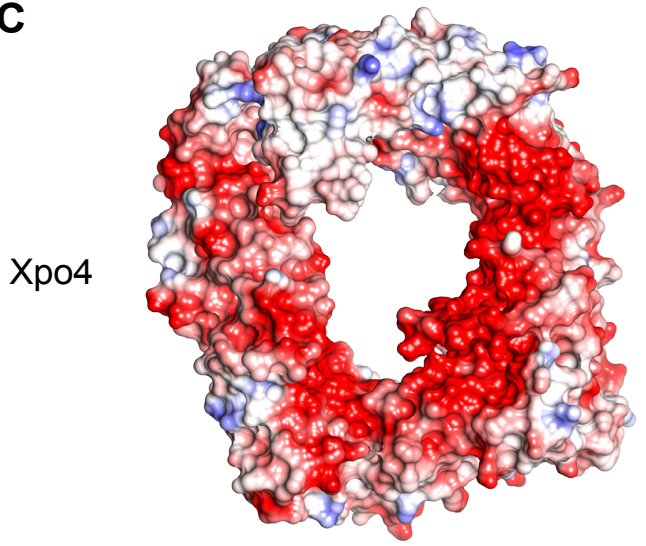

elF5A

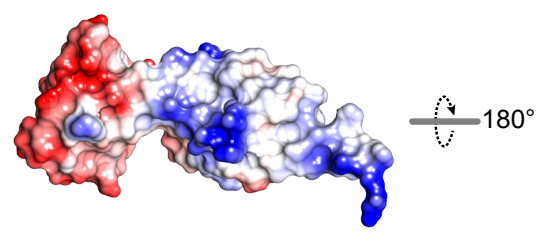

B

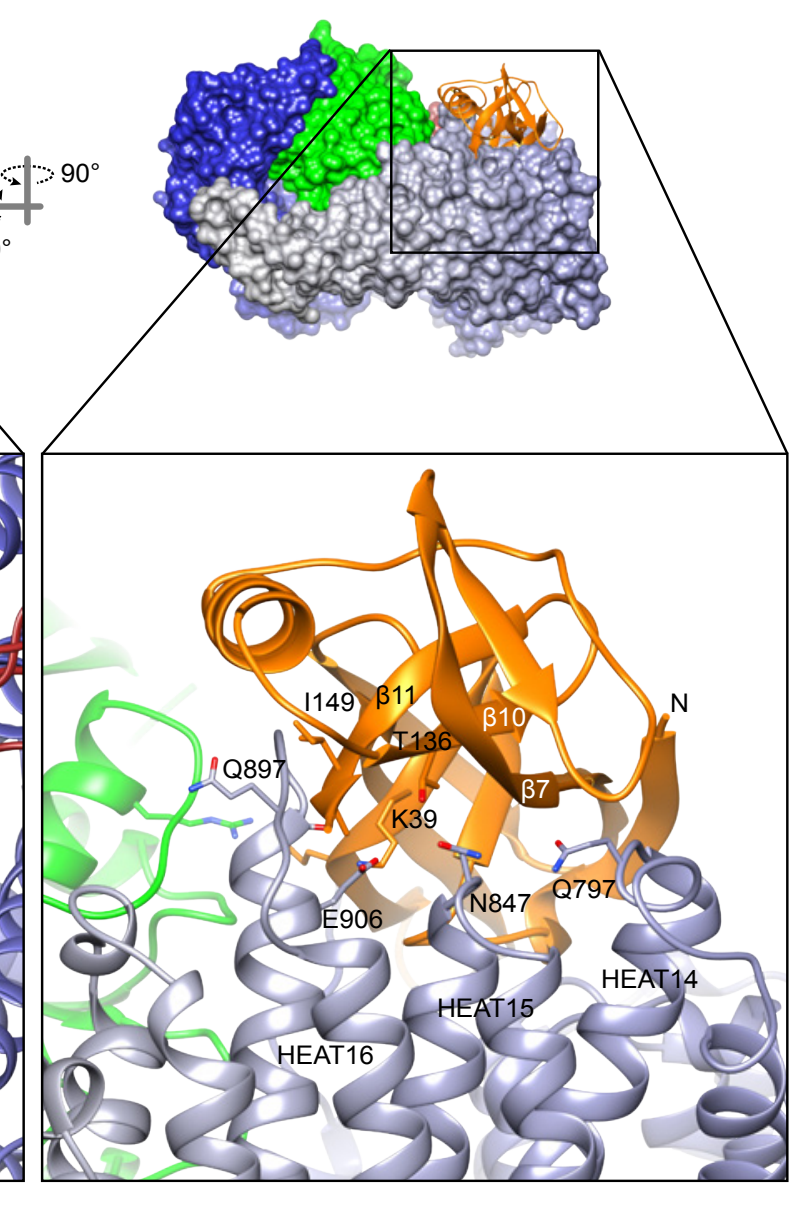


The N-terminal SH3-like domain of elF5A constitutes the larger interaction interface and contacts several residues on HEATs 8 to 16 of Xpo4. Most of the interactions are governed by a number of salt bridges and hydrogen bonds. The basic tip of elF5A, composed of $\beta$ strand 3 ( $\beta 3$ ), the hypusine-containing loop and $\beta$ strand 4 ( $\beta 4$ ) forms the center of the interactions (Figure 3-28a). The acidic loop of Xpo4 aligns next to $\beta 3$ and makes backbone hydrogen bonds with the residues of $\beta 3$ as if it were an antiparallel $\beta$ sheet and locks the basic tip of elF5A. In addition, Glu462 and Glu465 of the Xpo4 acidic loop contact Thr45, Thr48 and Lys55 on the basic tip of elF5A and further stabilize this interaction. Similarly, the hypusine-containing loop engages in direct interactions with the residues of HEATs 8 to 11. The hypusine (Hpu50) side chain bends in an L shape and seems to hook into the acidic loop (Figure 3-27 and Figure 3-29). This hook is positioned by several acidic residues of Xpo4. In particular, the amine nitrogen (terminal nitrogen) and hydroxyl oxygen of the butylamine moiety of hypusine interact with $\mathrm{Xpo4}^{\mathrm{Glu} 390}$ and $\mathrm{Xpo} 4^{\mathrm{Asp} 470}$, respectively. In addition, the side chain nitrogen $(\varepsilon$ nitrogen) of hypusine is stabilized by $\mathrm{Xpo}^{\mathrm{Asp} 470}$ and $\mathrm{Xpo4} 4^{\mathrm{Glu537}}$. Likewise, elF5A ${ }^{\text {His51 }}$ of the loop contacts Xpo $4^{\text {Glu537 }}$ and Xpo $4^{\text {Ser631 }}$ via nitrogens of imidazole side chain. Finally, $\beta 4$ of the basic tip is positioned by polar interactions between the intra-repeat loops of HEATs 11 and 12 Figure 3-28.
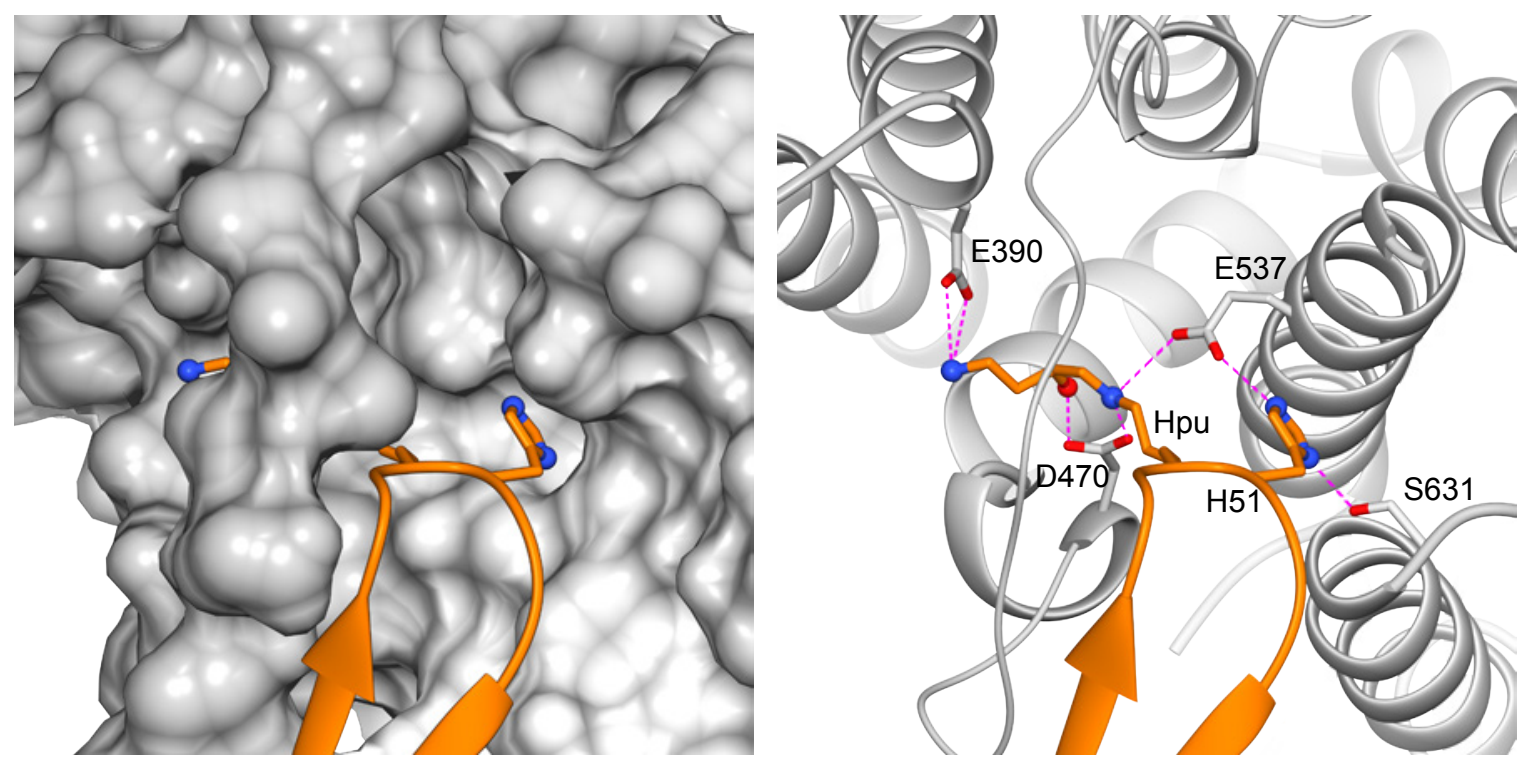

Figure 3-29 Details of the interactions in the acidic pocket

Docking of hypusine-containing loop into the acidic pocket of Xpo4 is shown. elF5A is shown as orange ribbon, while the hypusine (Hpu) and histidine (H51) are shown as sticks. Xpo4 is colored in grey and depicted as surface representation on the left and as ribbon on the right. The Xpo4 residues that interact with the hypusine and the histidine are shown as sticks. Nitrogen and oxygen atoms are shown as blue and red spheres, respectively. (Adapted from Aksu et al., 2016) 
The C-terminal OB-fold domain of elF5A forms the smaller interaction surface, but engages in extensive interactions with Xpo4. C-terminal domain sits on a concave surface made up of the intra-repeat loops of HEATs 14 to 16 (Figure 3-28b). Intrarepeat loop of HEAT 16 runs in the opposite direction to $\beta$ strand 11 ( $\beta 11)$ and makes backbone hydrogen bonds as well as hydrophobic contacts via elF5A $A^{\text {lle149. }}$. Several residues of the intra-repeat loops of HEATs 14 and 15 approach elF5A and interact with $\beta$ strand 7 and 10 ( $\beta 7$ and $\beta 10$, respectively) via polar contacts.

Finally, elF5A does not only interact with Xpo4 but also with Ran. Glu42 of elF5A contacts Ran via Arg29 (Figure 3-28a).

\section{Analysis of the structure with biochemical and functional assays}

As described in the previous sections, Xpo4 has very large and different interaction interfaces with both Ran and elF5A. To analyze the importance of these interfaces, I designed Xpo4 mutants that would block either Ran or elF5A binding.

\section{Xpo4 mutants that block RanGTP binding}

I analyzed the three Ran-binding interfaces of Xpo4, by creating the following Xpo4 mutants: Y110R (interface 1); D395R, D396R and D401R (interface 2); E905R and E906R (Interface 4), including double and quadruple mutations at the interface 2 and 4 (Figure 3-30). Wild type Xpo4 and arginine mutants were incubated with ZZ-NEDD8 tagged RanGTP. After Ran was recovered on anti-Z affibody dimer beads, Ran and bound proteins were eluted with bdNEDD8 protease. The sample from inputs and eluates were analyzed by SDS-PAGE (Figure 3-31a). Despite slight differences on bound Xpo4, none of the interface 2 and 4 mutations (including the quadruple mutation) blocked Xpo4 binding. On the contrary, the Xpo4 ${ }^{\mathrm{Y} 110 \mathrm{R}}$ mutation significantly impaired Ran binding. To rule out the possibility that this result was due to the side effects like impaired folding, I performed a binding assay with an import cargo, Sox2. Wild type Xpo4 and arginine mutants were incubated with Sox2. After Xpo4 was recovered on phenylsepharose beads, Xpo4 and bound proteins were eluted with SDS and analyzed by SDS-PAGE (Figure 3-31b). All mutants were able to bind the phenyl-sepharose beads and co-purified Sox2, indicating that a proper folding of Xpo4 was retained in these mutants. 

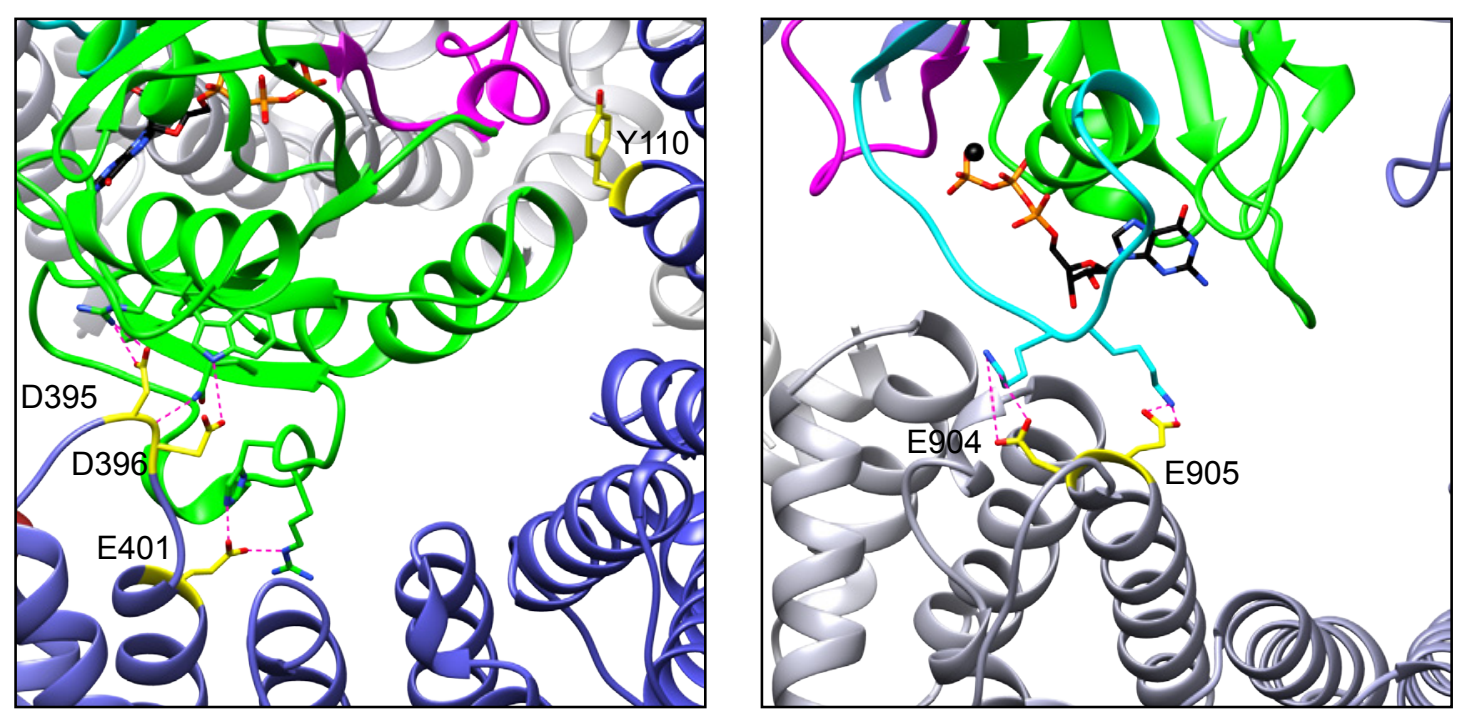

Figure 3-30 Ran-binding interfaces of Xpo4

Two images show the molecular details of RanGTP recognition by Xpo4. The export complex is shown in same color-coding as in Figure 3-25, with the mutated residues of Xpo4 shown in yellow. 


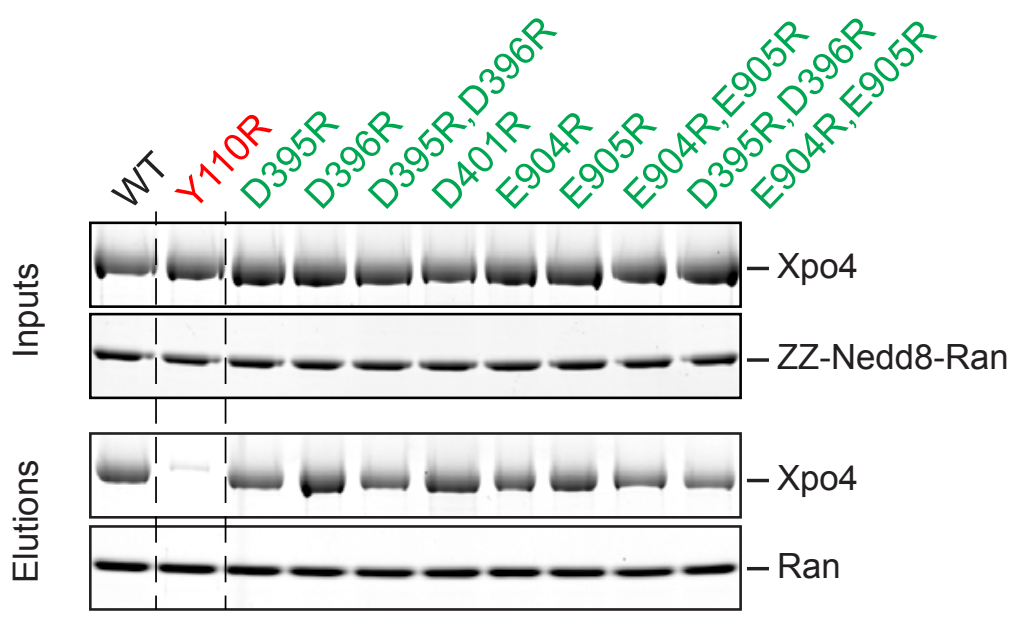

B Binding of Xpo4 variants to phenyl-sepharose beads

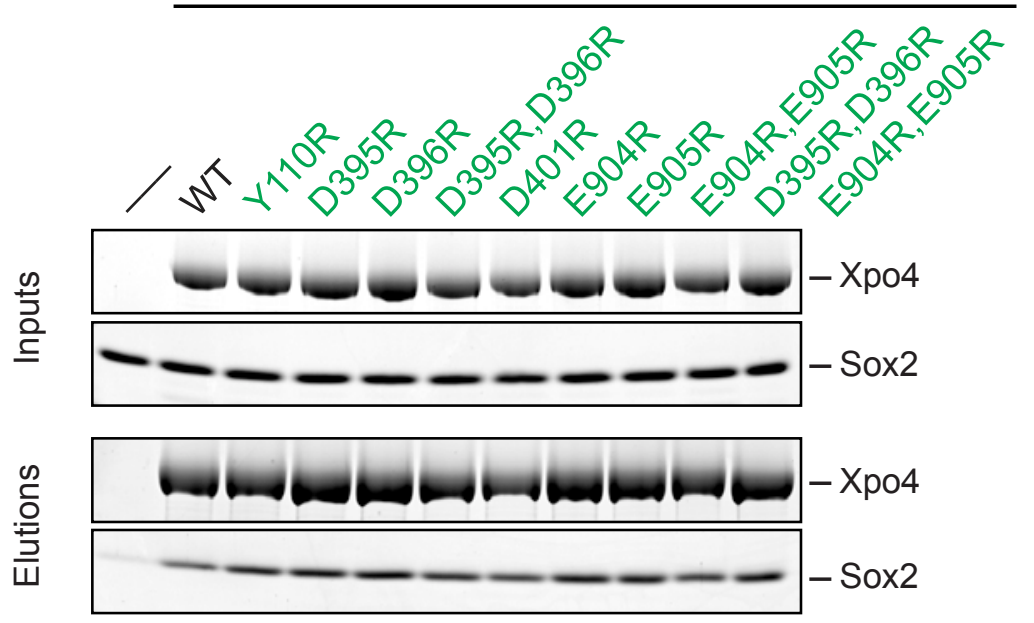

Figure 3-31 $\mathrm{N}$ terminal interaction interface of Xpo4 is essential for RanGTP binding

(A) $0.75 \mu \mathrm{M}$ ZZ-Nedd8 tagged RanGTP was mixed with $1 \mu \mathrm{M}$ wild type or mutant Xpo4 in $350 \mu \mathrm{L}$ at $100 \mathrm{mM}$ $\mathrm{NaCl}$. After $2 \mathrm{~h}$ incubation at $4{ }^{\circ} \mathrm{C}, 300 \mu \mathrm{L}$ of the samples was incubated with anti-Z affibody dimer beads to capture RanGTP and bound proteins. After another $2 \mathrm{~h}$ incubation, unbound proteins were removed, RanGTP and bound proteins were eluted by incubating the beads with $250 \mathrm{nM}$ bdNEDD 8 protease. Both inputs and eluates were analyzed by SDS-PAGE followed by Coomassie staining. (B) The samples were prepared by mixing $2 \mu \mathrm{M}$ Sox2 fragment with $0.75 \mu \mathrm{M}$ wild type or mutant Xpo4 in $350 \mu \mathrm{L}$ buffer at $100 \mathrm{mM} \mathrm{NaCl}$. After $2 \mathrm{~h}$ incubation at $4{ }^{\circ} \mathrm{C}, 300 \mu \mathrm{L}$ of the samples was incubated with phenyl-sepharose beads to recover Xpo4 and bound proteins. Unbound proteins were removed; Xpo4 and the bound proteins were eluted with SDS sample buffer. The inputs and eluates were analyzed by SDS-PAGE followed by Coomassie blue staining. A sample without Xpo4 served as negative control for Sox2 binding to the beads.

Overall, these results proved that the $\mathrm{N}$-terminal interaction interface (interface 1) of Xpo4 is essential for RanGTP binding. The results were also inline with the hypothesis that in the absence of elF5A, Xpo4 might have a different conformation than that in the export complex. To test this idea, I performed the same RanGTP 
binding experiment in the presence of hypusinated elF5A (Figure 3-32). The double mutation at the second interface (Xpo4 $4^{\mathrm{D} 395 \mathrm{R}, \mathrm{D} 396 \mathrm{R}}$, interacting with the back of Ran) impaired elF5A binding. Moreover, the quadruple mutation (Xpo4 ${ }^{\mathrm{D} 395 \mathrm{D}, \mathrm{D} 396 \mathrm{R}, \mathrm{E} 904 \mathrm{R}, \mathrm{E} 905 \mathrm{R}}$, interacting with the back and the switch I of Ran) further weakened elF5A binding, indicating that correct positioning of the Ranbinding interfaces are required for efficient elF5A binding.

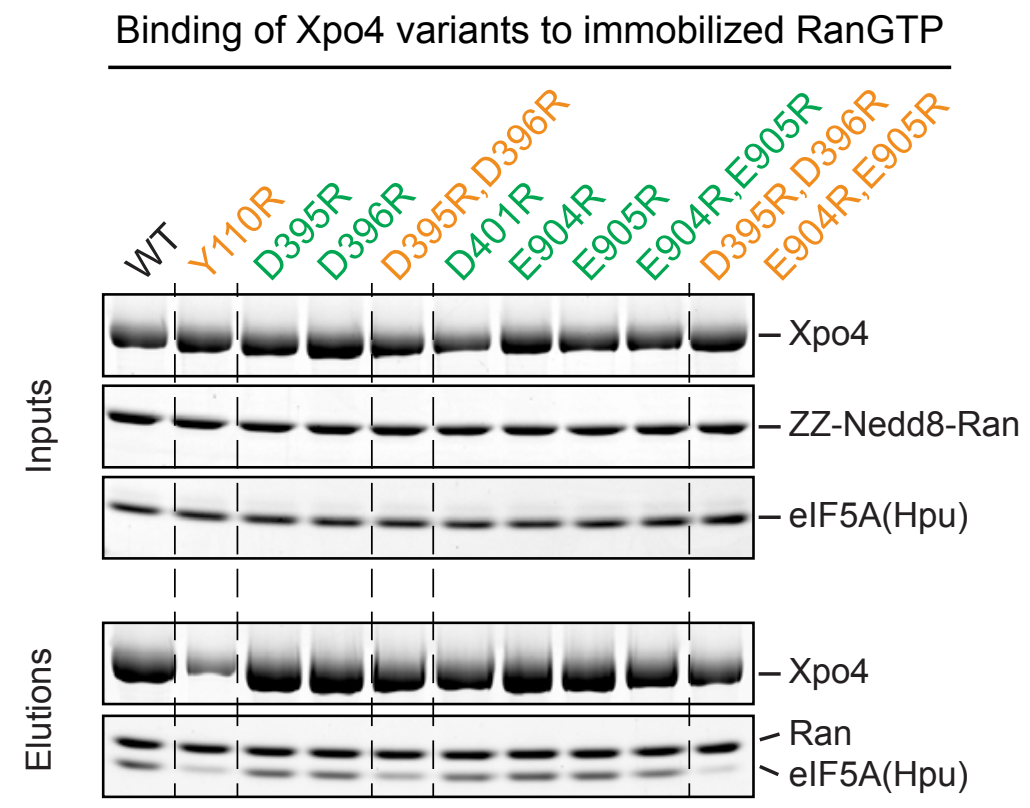

Figure 3-32 Ran-binding interface 2 and $\mathbf{4}$ are required for proper elF5A binding

$1 \mu \mathrm{M}$ wild type or mutant Xpo4 was mixed with $1 \mu \mathrm{M}$ hypusinated elF5A and $0.75 \mu \mathrm{M}$ ZZ-Nedd8 tagged RanGTP in $350 \mu \mathrm{L}$ at $100 \mathrm{mM} \mathrm{NaCl}$. After $2 \mathrm{~h}$ incubation at $4{ }^{\circ} \mathrm{C}, 300 \mu \mathrm{L}$ of the samples was incubated with Zaffibody dimer beads to capture RanGTP and bound proteins. After another $2 \mathrm{~h}$ incubation, unbound proteins were removed, RanGTP and bound proteins were eluted by incubating the beads with 250 nM bdNEDD8 protease. Both, inputs and eluates were analyzed by SDS-PAGE followed by Coomassie staining. Note that the binding strength of Xpo4 variants (including Y110R mutant) to RanGTP increases in the presence of elF5A (when compared to Figure 3-31), beautifully demonstrating the positive influence of an export cargo on the affinity of an exportin to RanGTP.

\section{Xpo4 mutants that block elF5A binding}

The recognition of elF5A by Xpo4 is complex and includes three-dimensional features of elF5A. Especially, the basic tip of elF5A extensively interacts with Xpo4. To test the requirements of these interactions, I created another set of Xpo4 mutants and tested their ability for RanGTP-dependent elF5A binding (Figure 3-33). 

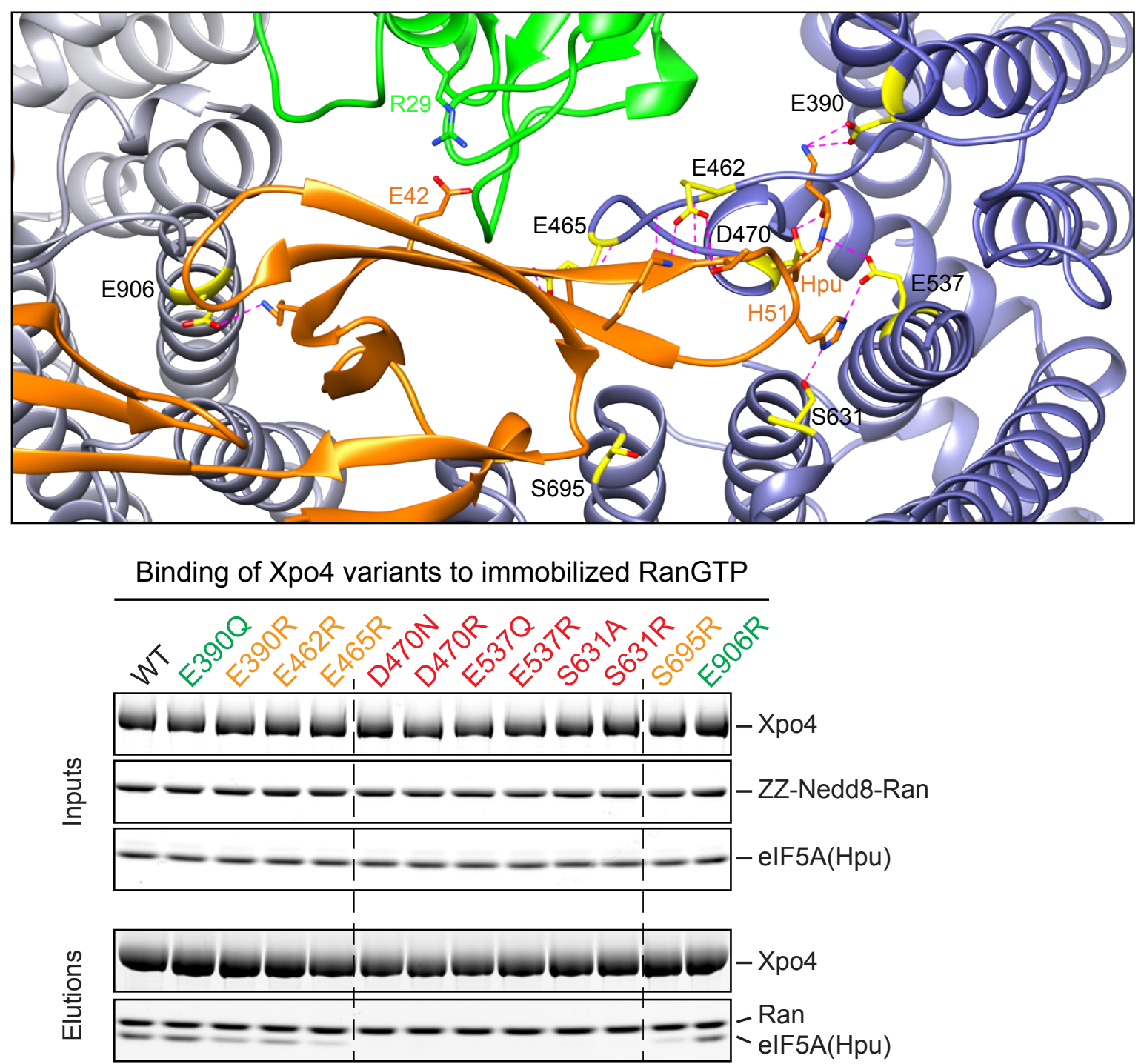

Figure 3-33 N terminal docking of elF5A is crucial for Xpo4 binding

Upper panel. Xpo4 elF5A interaction interface is shown in a similar view and color-coding as in Figure 3-28, with the mutated residues of Xpo4 shown in yellow. The residues of elF5A and Ran that are mentioned in the text are colored in orange and green, respectively. Lower panel. $1 \mu \mathrm{M}$ Xpo4 wild type or mutants was incubated with $0.75 \mu \mathrm{M}$ ZZ-brNEDD8 tagged RanGTP and $1.25 \mu \mathrm{M}$ hypusinated elF5A in a buffer containing $100 \mathrm{mM} \mathrm{NaCl}$. Experiment was performed essentially similar to Figure 3-32. (Adapted from Aksu et al., 2016)

$\mathrm{Xpo} 4^{\mathrm{D} 470 \mathrm{R}}, \mathrm{Xpo}^{\mathrm{E5} 37 \mathrm{R}}$ and $\mathrm{Xpo} 4^{\mathrm{S} 631 \mathrm{R}}$ mutations at the acidic pocket completely abolished elF5A binding, whereas Xpo4 ${ }^{\mathrm{E} 390 \mathrm{R}}, \mathrm{Xpo}^{\mathrm{E} 465 \mathrm{R}}$ and $\mathrm{Xpo} 4^{\mathrm{S} 695 \mathrm{R}}$ mutations severely reduced it. All these mutated residues would sterically clash with elF5A, but still allowed binding of RanGTP, indicating that the mutations did not affect Xpo4 folding. In addition, I tested much milder set of mutations (Xpo4 ${ }^{\mathrm{D} 40 \mathrm{~N}}$, $\mathrm{Xpo} 4^{\mathrm{E} 537 \mathrm{Q}}$ or $\mathrm{Xpo} 4^{\mathrm{S} 631 \mathrm{~A}}$ ), not aiming at steric exclusion, but loss of interactions. The loss of interaction mutants of Xpo4 also impaired export complex formation while still binding to RanGTP. Considering the extensive interactions between elF5A and Xpo4, it was unexpected to observe loss of elF5A binding with single $\mathrm{Xpo} 4^{\mathrm{D} 470 \mathrm{~N}}, \mathrm{Xpo}^{\mathrm{E} 537 \mathrm{Q}}$ or $\mathrm{Xpo4^{ \textrm {S631A } }}$ mutants. To verify these results, I performed 
the binding experiments the other way around using elF5A mutants (elF5A $\mathrm{A}^{\mathrm{K} 5 \mathrm{~A}}$, elF5A ${ }^{\mathrm{K} 50 \mathrm{R}}$, elF5A $\mathrm{H}^{\mathrm{H1A}}$; Figure 3-34). Likewise, none of the mutants bound to Xpo4. These results indicated that the interaction of the hypusine-containing loop is essential for elF5A binding to Xpo4•RanGTP.

Binding of Xpo4•RanGTP to immobilized elF5A variants

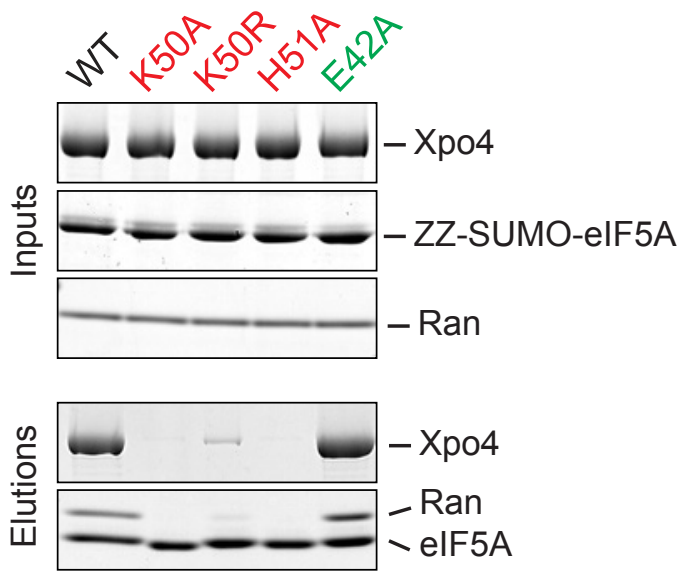

Figure 3-34 Hypusine-containing loop is essential for Xpo4 binding

$0.75 \mu \mathrm{M}$ wild type or mutant His14-ZZ-SUMO tagged non-hypusinated elF5A was mixed with $1 \mu \mathrm{M}$ Xpo4 and RanGTP in $350 \mu \mathrm{L}$ at $50 \mathrm{mM} \mathrm{NaCl}$. After $2 \mathrm{~h}$ incubation at $4{ }^{\circ} \mathrm{C}, 300 \mu \mathrm{L}$ of the samples was incubated with Zaffibody dimer beads to capture RanGTP and bound proteins. After another $2 \mathrm{~h}$ incubation, unbound proteins were removed, RanGTP and bound proteins were eluted by incubating the beads with $250 \mathrm{nM}$ bdSUMO protease. The inputs and eluates were analyzed by SDS-PAGE followed by Coomassie staining.

The above-described experiments showed that the N-terminal docking of elF5A into the acidic pocket was necessary for elF5A binding. However, the Xpo4 ${ }^{\mathrm{E} 390 \mathrm{R}}$ mutation, which would block the N-terminal insertion, did not completely impaired elF5A binding (Lane 3 in Figure 3-33). There were two possible reasons for this observation. First, the tip of the hypusine or R390 residue could be flexible, which would allow binding of elF5A by adopting a different conformation. Second, model building of the hypusine might be wrong. To test these two hypotheses, I performed an experiment where elF5A variants (hypusine, deoxyhypusine or lysine) were tested for $\mathrm{Xpo} 4^{\mathrm{E} 390 \mathrm{R}}$ binding. As positive and negative controls, I included wild type and D470N mutant Xpo4. Figure 3-35 shows that the binding strength of elF5A variants to wild type Xpo4 correlates with the modification state of elF5A; on the other hand, independent of the modification state, the D470N mutation prevented binding of all elF5A variants. The binding of hypusinecontaining elF5A to Xpo4 decreased in the E390R mutant. The decrease was more prominent in the case of deoxyhypusine-containing elF5A. Remarkably, the binding was restored in lysine-containing elF5A. Taken together, these results 
proved that the butylamine moiety of hypusine resides in close proximity to the E390 residue of Xpo4.
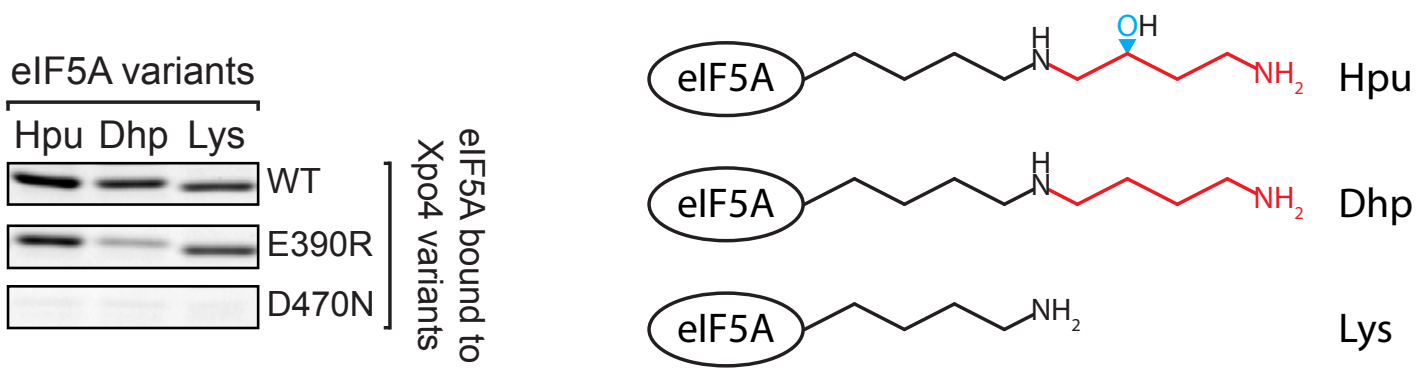

Figure 3-35 Interaction of E390 with the hypusine

Experimental setup is essentially similar to Figure 3-33. Hpu, Dhp and Lys denote hypusine, deoxyhypusine and lysine, respectively.

Finally, I investigated the functionality of the Xpo4 mutants with a nuclear export assay. For the assay, I used digitonin-permeabilized HeLa cells. Digitonin treatment permeabilizes the plasma membrane while keeping the nuclear membrane intact (Adam et al., 1990) and therefore allows introduction of probes into the cells. Moreover, since most of the soluble nuclear transport factors are washed away during the permeabilization process, active transport depends on addition of the soluble components back to the permeabilized cells. As a result, this assay allows examination of individual components, such as nuclear transport receptors. Additionally, the permeabilized cells are stable and functional for hours in the presence of Xenopus egg extract (Stuven et al., 2003). In the assay, I used an egg extract that had been depleted of NTRs by phenyl sepharose (Ribbeck and Görlich, 2002). The assay made use of the fact that the small size of elF5A allowed a fast passive diffusion into the nuclei. elF5A has one surface-exposed cysteine residue, which is not in an interface with Ran and Xpo4 and therefore does not affect Xpo4 binding. As a result, elF5A can be quantitatively labeled with maleimide dyes. As seen in Figure 3-36, fluorescently-labeled elF5A diffused into the nuclei and got enriched in the nucleoli after $15 \mathrm{~min}$ incubation. These incubations were split and further incubated with wild type Xpo4 or with Xpo4 mutants. Wild type Xpo4 efficiently exported the diffused elF5A and cleared the nucleolar signal. The mutations $\left(\mathrm{Xpo} 4^{\mathrm{E} 390 \mathrm{R}}, \mathrm{Xpo} 4^{\mathrm{E} 462 \mathrm{R}}\right.$ and $\mathrm{Xpo} 4^{\mathrm{E} 465 \mathrm{R}}$, orange labels in Figure 3-33) that reduced elF5A binding did not eliminate the export activity, suggesting that Xpo4 could function efficiently even if the binding strength was reduced. On the contrary, the mutations (Xpo4 ${ }^{\mathrm{D} 470 \mathrm{~N}}, \mathrm{Xpo} 4^{\mathrm{E} 537 \mathrm{Q}}$ and $\mathrm{Xpo} 4^{\mathrm{S} 631 \mathrm{~A}}$, 
red labels in Figure 3-33) that completely abolished elF5A binding also blocked the export activity of Xpo4.

15 min diffusion

Further incubation with Xpo4 variants
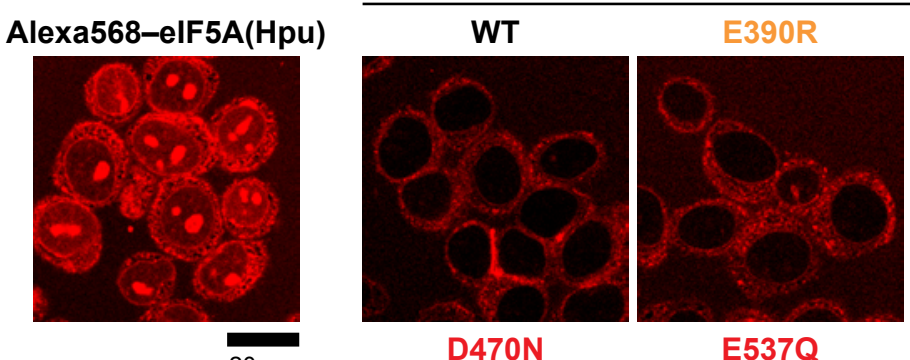

E462R

E465R

$20 \mu \mathrm{m}$

D470N

E537Q

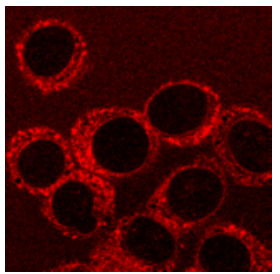

S631A
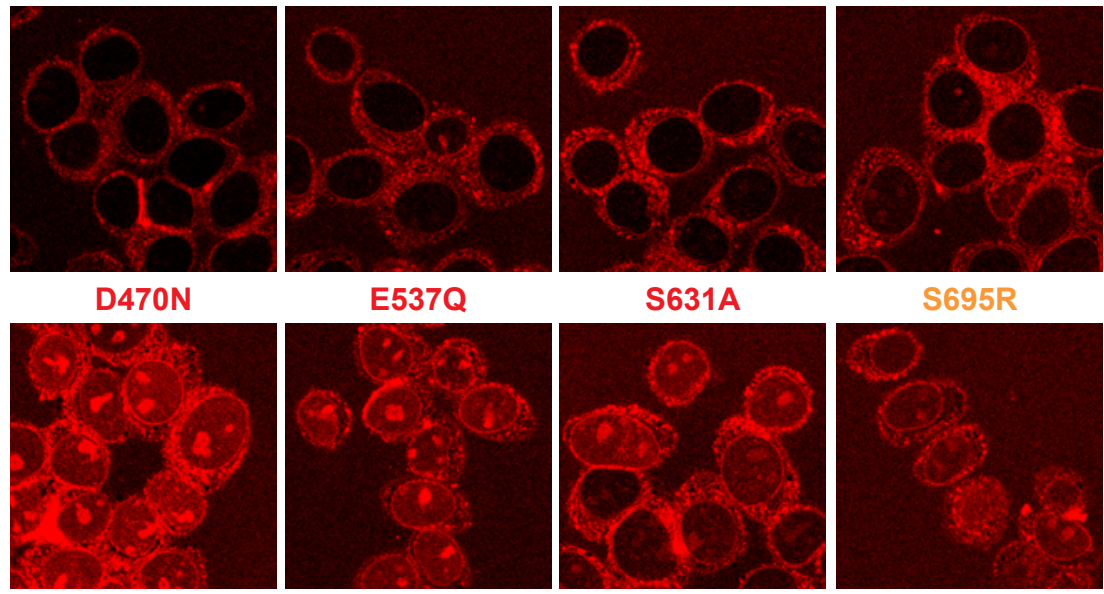

S695R

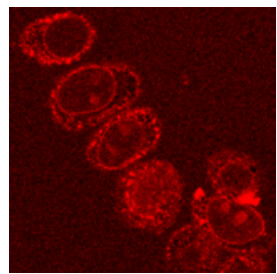

Figure 3-36 $\mathrm{N}$ terminal docking of elF5A is also essential for export activity of Xpo4

Alexa567-labelled hypusinated elF5A was allowed to diffuse into the nuclei of permeabilized HeLa cells in the presence of an energy-regenerating system and a Xenopus egg extract that importin $\beta$-like transport receptors had depleted. After $15 \mathrm{~min}$, the mixture was split and $2 \mu \mathrm{M}$ Xpo4 variant was added. After 30 min, the distribution of elF5A was recorded by confocal fluorescence microscopy. (Adapted from Aksu et al., 2016)

\subsubsection{Crystallization trials of the Sox2 import complex}

Crystallization of the Sox2 import complex, composed of Sox2 and Xpo4, was the second task of this project. In spite of the extensive effort, we were not able to solve the structure of this complex. However, the knowledge gained from Xpo4 during the crystallization of the export complex led us to obtain import complex crystals. In this section, I will briefly summarize the results that guided us to the crystals.

Bacterial expression and reconstitution of full-length Xpo4 and Sox2 resulted in a stoichiometric complex. The import complex was stable up to $500 \mathrm{mM}$ salt concentration (data not shown, see Figure 3-4 for comparison with the export complex). MALS analysis indicated that the molecular weight of the import complex was $167 \mathrm{kDa}$, suggesting a 1:1 stoichiometry (Figure 3-37). MALS analysis also revealed an interesting feature of the import complex. Although the molecular weights of the import and the export complexes were almost identical, the import complex eluted from the gel filtration column earlier. This suggested that the export complex was more compact than the import complex. 


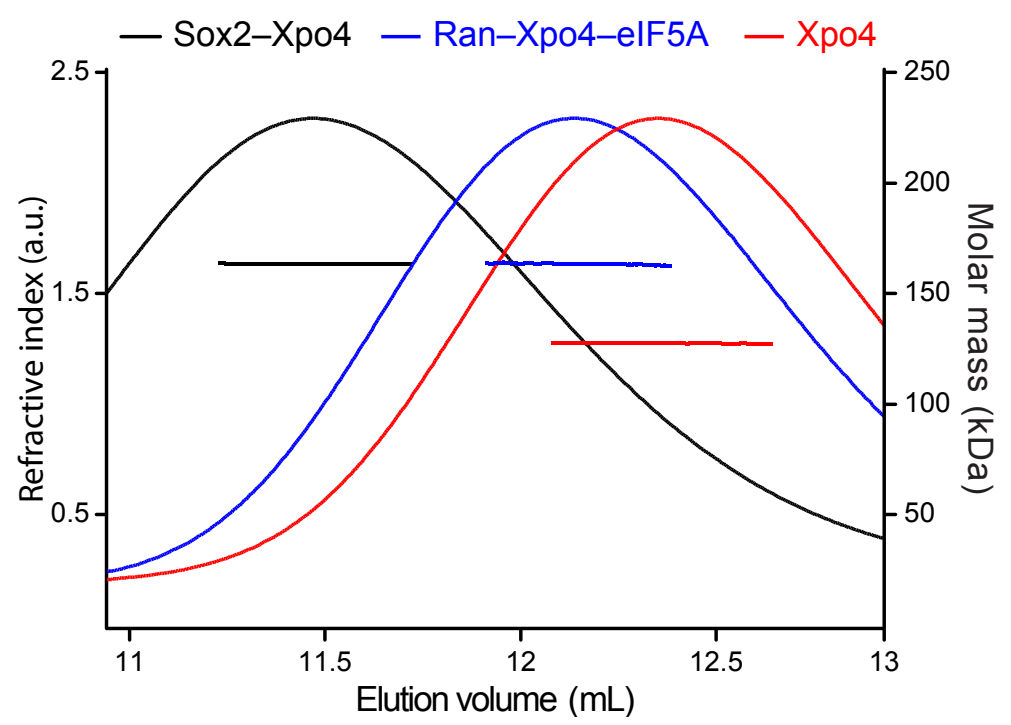

Figure 3-37 MALS analysis of Xpo4 complexes

Gel filtration-purified complexes of Sox2-Xpo4 (black), RanGTP-Xpo4-elF5A ${ }^{15-154}$ (blue) and Xpo4 (red) were concentrated to $2 \mathrm{mg} / \mathrm{mL}$ and analyzed by Superdex $20010 / 30$ column coupled to MALS detector. Theoretical molar masses of the complexes are $166 \mathrm{kDa}$ for Sox2-Xpo4, $165 \mathrm{kDa}$ for RanGTP-Xpo4-elF5A ${ }^{15-154}$, and $130 \mathrm{kDa}$ for $\mathrm{Xpo} 4$ alone.

Initial crystallization screenings with the full-length Xpo4-Sox2 complex failed. After the identification of the flexible loops in Xpo4, I tested binding of the Xpo4 deletion mutants (Xpo4 ${ }^{\Delta 241-260}, \mathrm{Xpo}^{\Delta 931-948}$ and $\mathrm{Xpo} 4^{\Delta 241-2602931-948}$ ) to Sox2. All of the proteins bound to Sox2 and formed stable import complexes. Nevertheless, I was not able to identify suitable crystallization conditions for the import complexes in spite of exhaustive screening.

The failure in the crystallization and the gel filtration profile again pointed out additional unstructured regions in the export complex. The aforementioned observation in the MALS experiment could be the result of Xpo4 adopting a more open conformation in the import cargo-bound state. Alternatively, possible unstructured regions of Sox2 might have led to the larger apparent size of the import complex. Intrinsically disordered regions are highly abundant in the eukaryotic transcription factors (Liu et al., 2006). In fact, secondary structure prediction algorithms find large regions of Sox2 to be disordered. Therefore, I aimed at finding the flexible region(s) of Sox2 in the import complex. In vitro limited proteolysis experiments were not successful. As a result, I used the following approach to find out the minimal Xpo4-binding region of Sox2.

Sox2 is prone to degradation when expressed in E. coli (Figure 3-38a). Normally, Sox2 was expressed with $\mathrm{N}$ - and $\mathrm{C}$-terminal tag so that a pure full-length protein 
could only be obtained after tandem affinity purification. As seen in Figure 3-38a, after purification only with C-terminal tag, full-length protein as well as several low molecular weight proteins were acquired. To find out the minimal Xpo4-binding region, I co-expressed Sox2 with H14-bdSUMO tagged Xpo4 and purified the receptor and bound proteins by immobilizing Xpo4 to a Ni (II) chelate matrix followed by protease elution. The eluate was analyzed by size exclusion chromatography to identify the fragments that would co-migrate with Xpo4. Although the injected proteins eluted in a single peak, SDS-PAGE analysis revealed that there were at least two different populations (Figure 3-38). In addition to the full-length Sox2, there was one major $\sim 15 \mathrm{kDa}$ protein co-migrating with Xpo4. MS analysis revealed this protein to be a Sox2 fragment, comprising of residues 1-122 (these were the residues between trypsin cleavage sites, actual boundary might be slightly different). This region corresponded to the high-mobility group box (HMG-box) domain (DNA-binding domain) of Sox2.

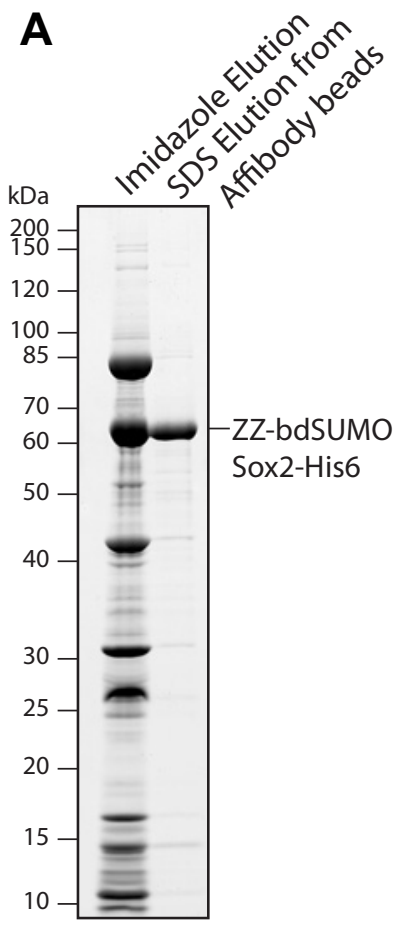

B

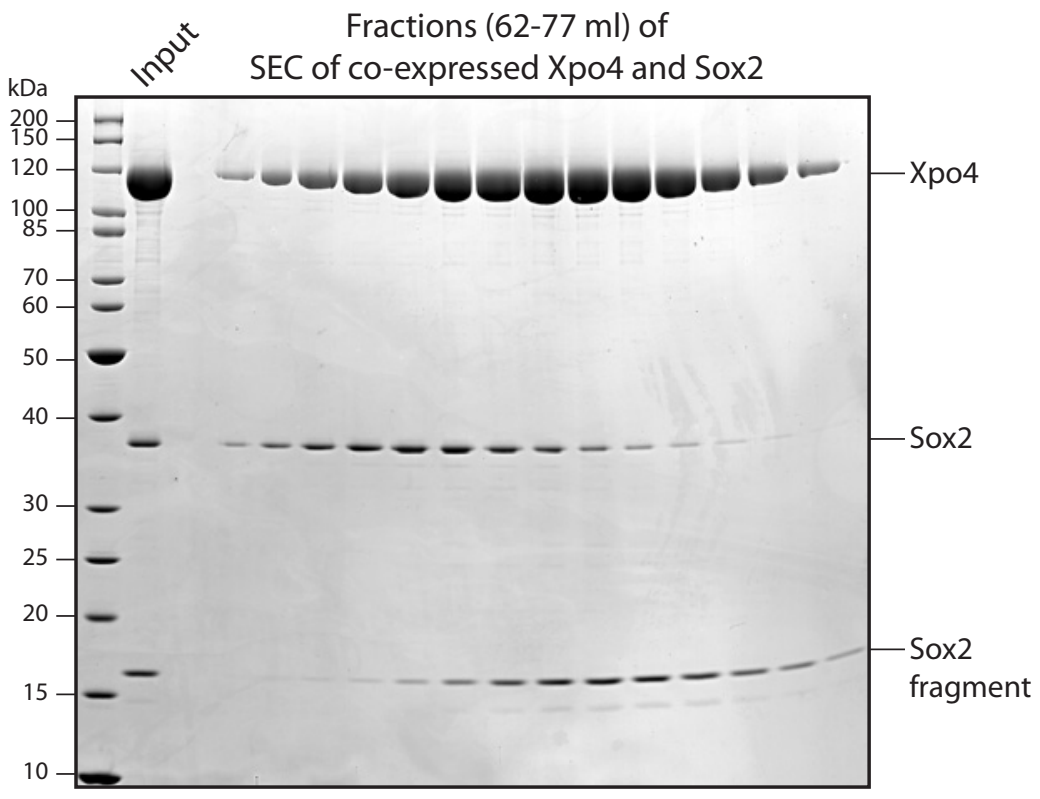

Figure 3-38 Identification of minimal Xpo4-binding region of Sox2

(A) N- and C- terminal tagged Sox2 was expressed in E. coli. Soluble lysate was loaded to Ni (II) chelate matrix. After removal of the unbound proteins, Sox2 fragments were eluted with imidazole. Many Sox2 fragments including a $85 \mathrm{kDa}$ chaperone were purified. The eluate was immobilized to anti-Z affibody dimer coupled beads and eluted with SDS. (B) Co-expressed proteins were purified via Xpo4 and subjected to size exclusion chromatography. Fragments corresponding to 62 to $77 \mathrm{ml}$ were analyzed by SDS-PAGE followed by Coomassie staining.

The HMG-box domain of Sox2 (residues 41-120) has been crystalized (Reményi et al., 2003). In addition, the residues 1-40 were disordered. Moreover, Gontan et 
al. (2009) revealed residues 38-113 to be part of the Xpo4-dependent import signal. Based on these studies and the result of MS analysis, I created a truncation construct of Sox2, consisting of residues 37-122. This fragment formed a stable import complex with Xpo4 (Figure 3-39). However, I again failed to identify any crystallization condition. Later, I also formed complexes with previously described Xpo4 deletion mutants. Nevertheless, the tested import complexes did not yield crystals.

After working with a minimal Sox2 fragment, I ascribed the failure in crystallization to Xpo4. We considered two possibilities for the failure. Either Xpo4 had additional unstructured loops that have to be removed or Xpo4 was so flexible that it prevented suitable crystal contacts. Analysis of the Xpo4 structure in the export complex did not indicate any further possible loops that can be removed. Due to its regulatory role in the export complex, we did not take the acidic loop into account. Therefore, we focused on the second possibility.

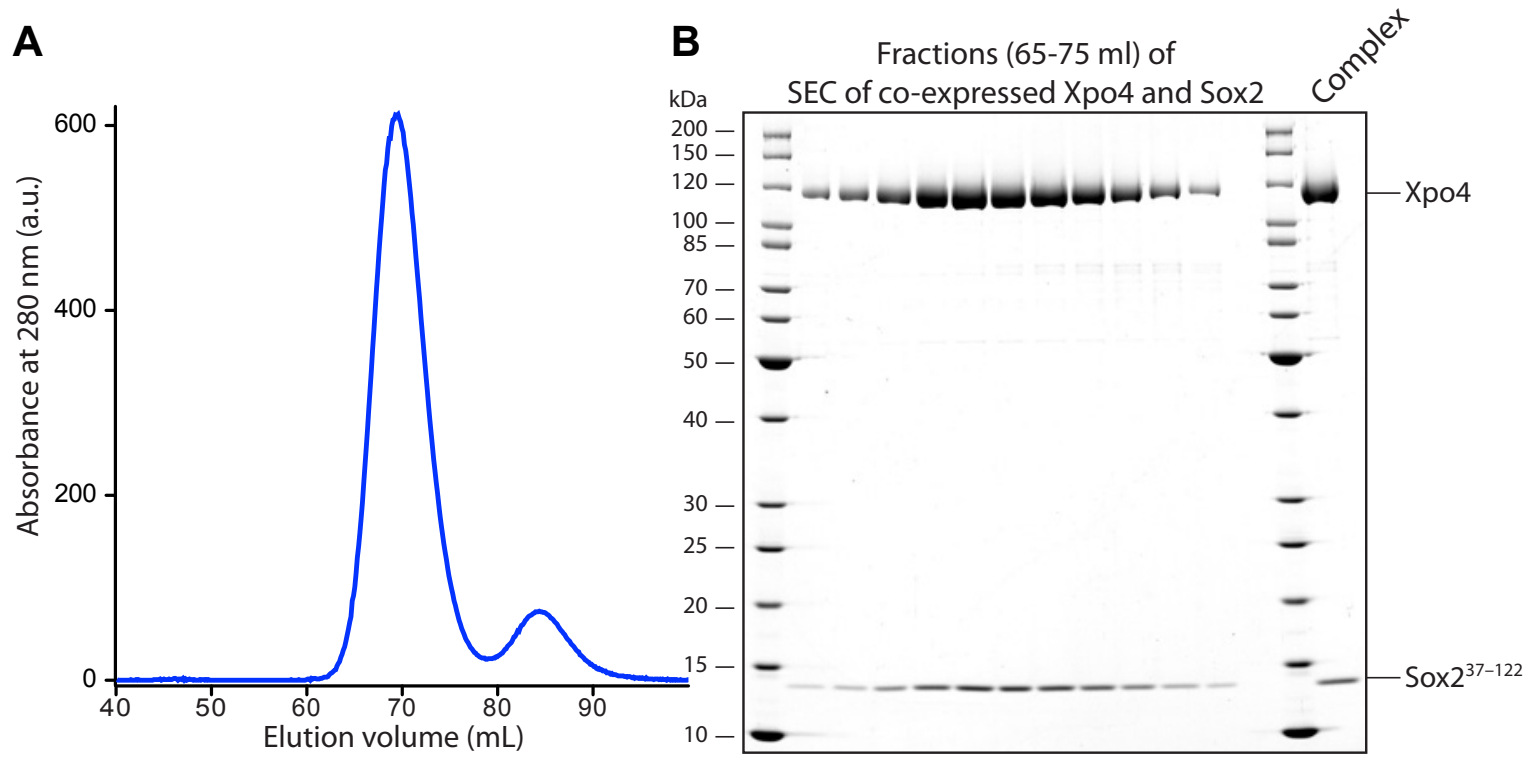

Figure 3-39 Reconstitution of the import complex with Sox2 fragment

(A) Gel filtration profile of the import complex. Xpo4 was mixed with Sox2 fragment, Sox2 being in excess. The complex was allowed to form in solution for $3 \mathrm{~h}$ at $4{ }^{\circ} \mathrm{C}$. Complex was separated from the excess Sox 2 by SEC on Superdex 200 16/60 column equilibrated with $20 \mathrm{mM}$ Tris/ $\mathrm{HCl} \mathrm{pH} \mathrm{7.7,100} \mathrm{mM} \mathrm{NaCl}$. Elutions were collected in $1.5 \mathrm{~mL}$ fractions. (B) $10 \mu \mathrm{L}$ of each fraction was analyzed by SDS-PAGE followed by Coomassie staining.

Crystallization requires the formation of well-ordered crystals. On the contrary, Impß-like NTRs are flexible due to their HEAT repeat structure. Two Xpo4 molecules in the asymmetric unit have already demonstrated the flexibility of Xpo4 (Figure 3-24). Further analysis of the Xpo4 structure revealed that the temperature 
factor (B-factor) of the $\mathrm{C}$-terminal region was higher than that of the rest of the structure (Figure 3-40). B-factor represents the uncertainty of an atom in the structure. Higher B-factors might be caused either by defects in the diffraction data or due to the different positioning of the atom in different unit cells of the crystal, which reflects the flexibility. Consequently, I decided to truncate Xpo4 from the C-terminus to obtain a relatively rigid structure.
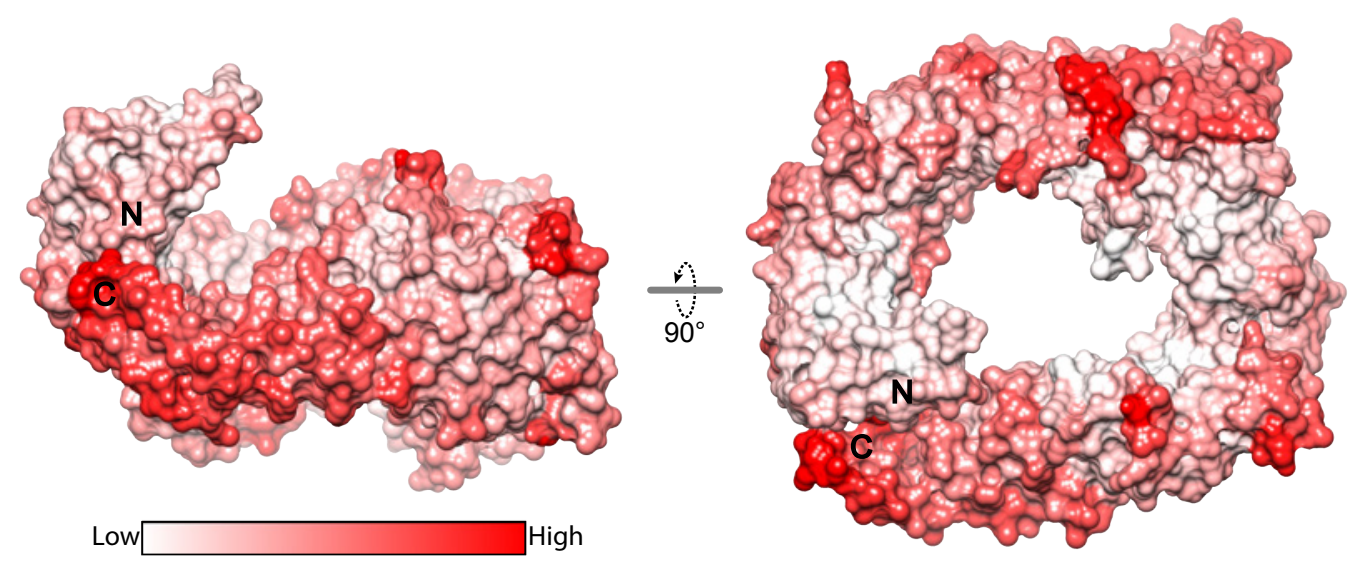

Figure 3-40 Surface of Xpo4 colored by B-factor

As described in the introduction (section 2.3), HEAT repeats form a continuous hydrophobic core, which is sealed by the terminal repeats. Therefore, the presence of the terminal repeats is required for proper folding of the NTR. Even removal of the last helix of the HEAT 20 (20C) resulted in complete insolubility of Xpo4 (see below). To obtain soluble proteins, I created several truncation constructs where the last helix was designed such that it ended with three or four hydrophobic residues, which could pack against the previous HEAT repeat and complete the folding of the molecule. The created Xpo4 truncations and their features are summarized in Table 3-4.

\begin{tabular}{|lcccc|}
\hline Xpo4 boundary & Last helix & Xpo4 solubility & Sox2 binding & Crystal formation \\
\hline $1-625$ & H11A & + & No & \\
\hline $1-691$ & H12A & ++ & Yes & Yes \\
\hline $1-759$ & H13B & + & Yes & \\
\hline $1-967$ & H17A & - & & \\
\hline $1-993$ & H17B & - & & \\
\hline $1-1069$ & H19A & - & & \\
\hline $1-1120$ & H20B & - & & No \\
\hline $1-1145$ & Full-length & ++++ & Yes & \\
\hline
\end{tabular}

Table 3-4 Mapping of Xpo4 boundaries for stable Sox2 binding

See also Figure 3-22 on page 61 for corresponding positions of the truncations. 
The constructs were expressed in E. coli. Soluble ones were tested for Sox $2^{37-122}$ binding. Among three soluble Xpo4 truncations, two of them bound to Sox2 fragment. The result implied that the HEAT repeat helices 11B or 12A could be part of the Sox2 recognition motif.

I formed an import complex with the minimal Xpo4 (Xpo4 ${ }^{1-691}$ ) and minimal Sox2 fragment $\left(\right.$ Sox $\left.2^{37-122}\right)$. The minimal import complex was separated by size exclusion chromatography and analyzed by SDS-PAGE and MALS (Figure 3-41). Final preparation was concentrated to 6 and $12 \mathrm{mg} / \mathrm{ml}$ and used for crystallization trials in the crystallization facility. Needle-like crystal clusters were observed in several conditions. These crystals resembled the initial crystals that we obtained from the export complex where the removal of the $\mathrm{N}$-terminus gave rise to diffracting quality crystals. Although, these needle-like crystals require further optimization, it was motivating to show that the import complex can be crystallized as well.

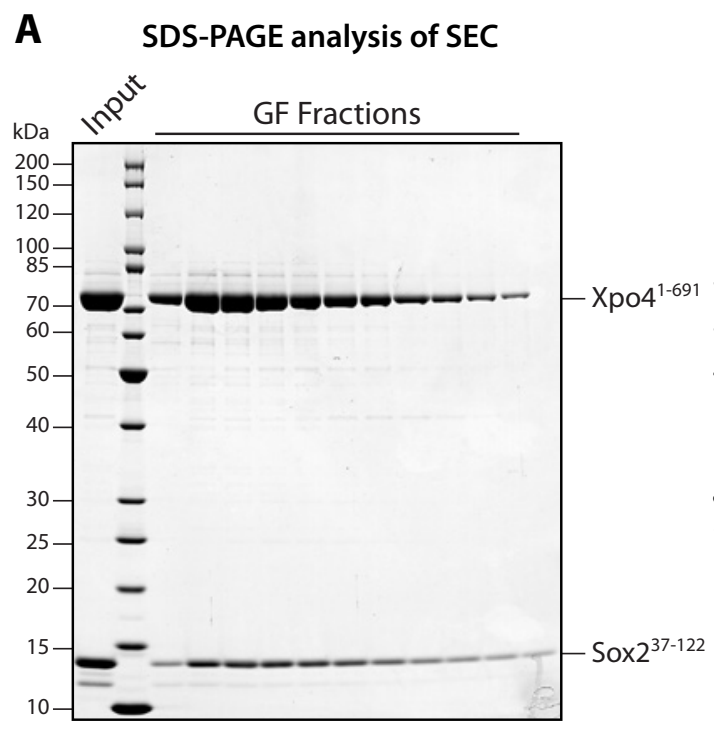

C

Sox $2^{37-122}-X p o 4^{1-691}$

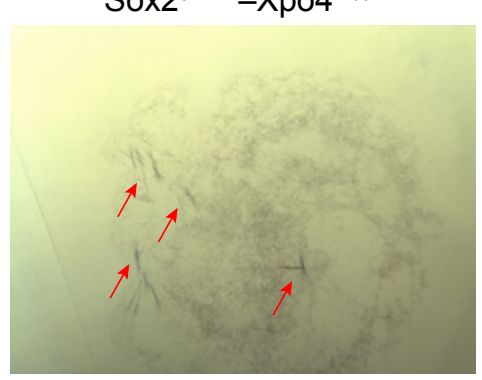

B

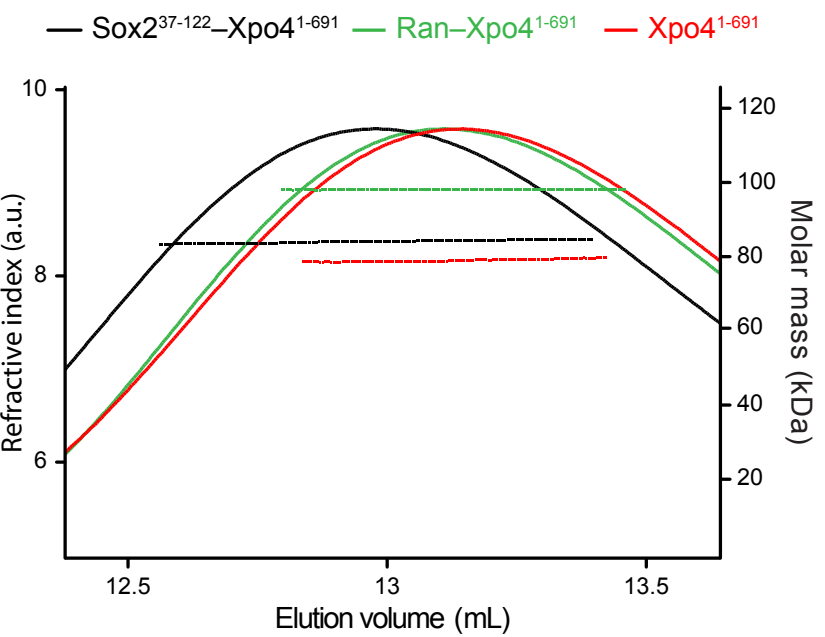

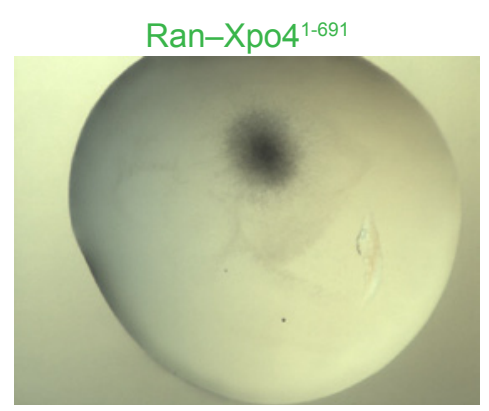

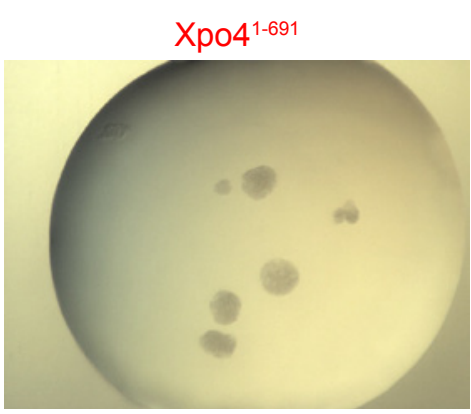

Figure 3-41 Reconstitution of the import complex with truncated Xpo4 and initial crystallization hits

(A) SDS-PAGE analysis of SEC of the minimal import complex. Xpo4 $4^{1-691}$ was mixed with Sox2 fragment, Sox2 being in excess. Complex was allowed form in solution for $3 \mathrm{~h}$ at $4{ }^{\circ} \mathrm{C}$. The complex was separated from the excess Sox2 by SEC on Superdex 200 16/60 column equilibrated with $20 \mathrm{mM} \mathrm{Tris/HCl} \mathrm{pH} \mathrm{7.7,} 100 \mathrm{mM}$ 
$\mathrm{NaCl}$. Elutions were collected in $1 \mathrm{~mL}$ fractions. $10 \mu \mathrm{L}$ of each fraction was analyzed by SDS-PAGE followed by Coomassie staining. Similar protocol was followed for RanGTP-Xpo $4^{1-691}$ and Xpo $4^{1-691}$ preparation. (B) Gel filtration-purified complexes of Sox2-Xpo4 (black), RanGTP-Xpo4 (green) and Xpo4 (red) were concentrated to $2 \mathrm{mg} / \mathrm{mL}$ and analyzed by Superdex 200 10/30 column coupled to MALS detector. Theoretical molar masses of the complexes were $89 \mathrm{kDa}$ for Sox2-Xpo4, $98 \mathrm{kDa}$ for RanGTP-Xpo4, and $79 \mathrm{kDa}$ for Xpo4 alone. All values are for truncated constructs. (C) After confirming the sample homogeneity by dynamic light scattering, protein samples were concentrated to 6 and $12 \mathrm{mg} / \mathrm{ml}$ and screened for crystallization hits. In several conditions, needle-like crystals or needle clusters were observed. One example for each protein sample is shown. Crystallization conditions of the drops were $100 \mathrm{mM} \mathrm{HEPES} \mathrm{pH} \mathrm{7.5,} \mathrm{8 \%} \mathrm{PEG} \mathrm{4000,}$ $100 \mathrm{mM} \mathrm{NaCl}$, and $50 \mathrm{mM} \mathrm{MgCl}_{2}$ for Sox2-Xpo4; 16\% PEG $800020 \%$ Glycerol, and $40 \mathrm{mM} \mathrm{KH}_{2} \mathrm{PO}_{4}$ for RanGTP-Xpo4; and $100 \mathrm{mM} \mathrm{NaH}_{2} \mathrm{PO}_{4} \cdot \mathrm{H}_{2} \mathrm{O}, 12 \%$ PEG 8000 for Xpo4.

\subsubsection{Crystallization trials of Xpo4 and Xpo4-RanGTP complex}

Crystallization of the elF5A export complex and Sox2 import complex required preparation of highly pure samples of Xpo4 and RanGTP. I used this opportunity to crystallize Xpo4 either alone or in complex with RanGTP. Complex formation of Xpo4-RanGTP was straightforward and similar to the protocol of the reconstitution of the export complex. Briefly, Xpo4 was mixed with H14-ZZ-bdSUMO tagged RanGTP in near stoichiometric ratio, Xpo4 being in excess in order to saturate the RanGTP. After the proteins were allowed to form complexes in solution, they were immobilized to the anti-Z affibody coupled matrix. Unbound proteins were removed and complex was eluted with protease elution. The eluate was analyzed by size exclusion chromatography followed by SDS-PAGE (Figure 3-42a, b). Injected proteins ran on the gel filtration as a single entity. The purity of Xpo4-RanGTP complex, as well as Xpo4, was high quality as judged by SDS-PAGE (Figure 3-42b, c) and by dynamic light scattering (data not shown). I used commercially available sparse matrix screens and set crystallization plates in the crystallization facility. By setting drops both at $4{ }^{\circ} \mathrm{C}$ and $20^{\circ} \mathrm{C}$, I tested approximately 2500 crystallization conditions. However, I couldn't detect any promising condition for the crystallization of Xpo4 or the Xpo4-RanGTP complex. 

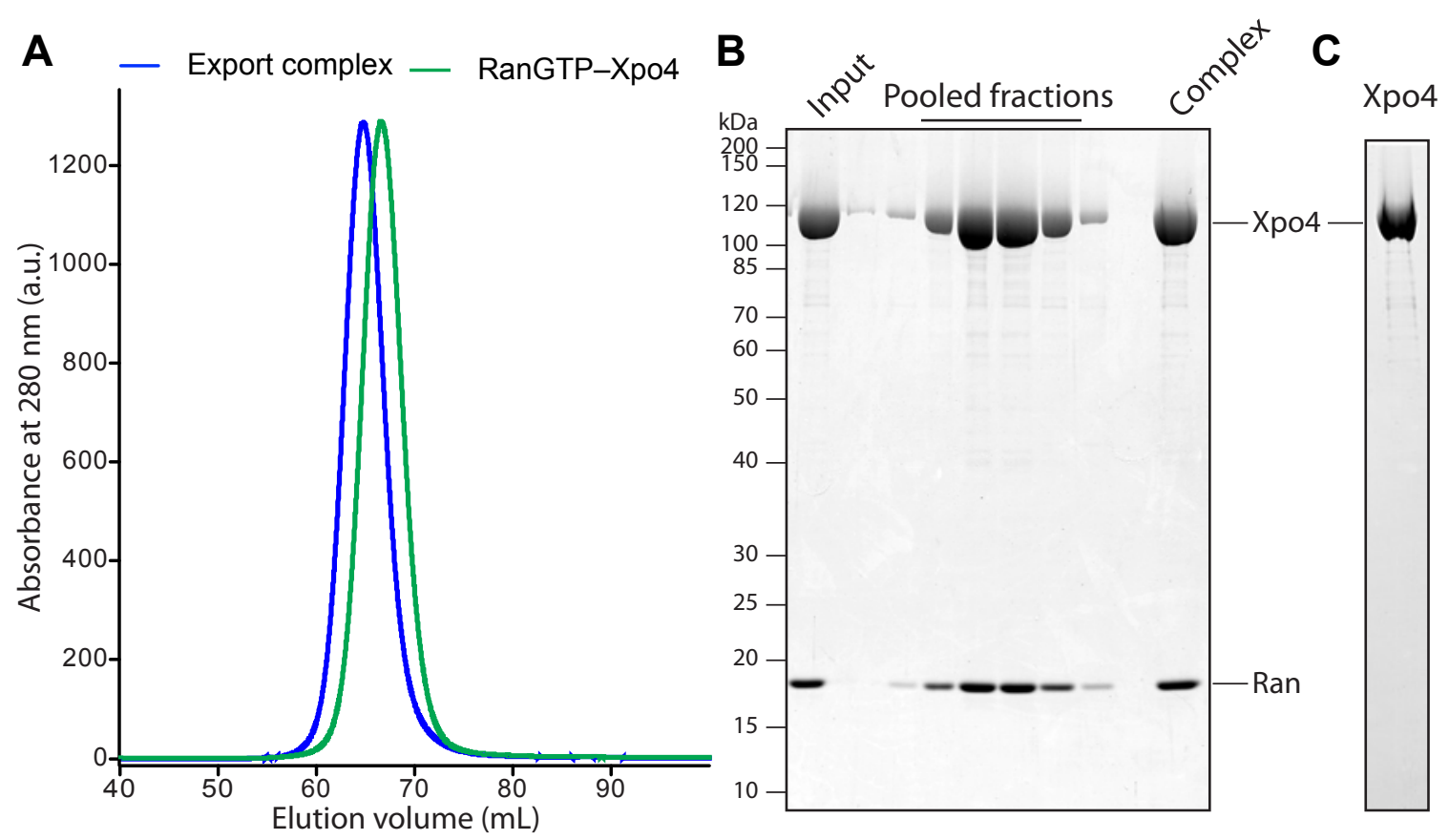

Figure 3-42 Reconstitution of RanGTP-Xpo4 complex

(A) Gel filtration profile of the RanGTP-Xpo4 on Superdex $20016 / 60$ column equilibrated with $15 \mathrm{mM}$ Tris/HCl $\mathrm{pH} 7.7,18 \mathrm{mM} \mathrm{NaCl}, 2 \mathrm{mM} \mathrm{Mg}(\mathrm{OAc})_{2}$ and $2 \mathrm{mM}$ DTT. Proteins were collected in $1.5 \mathrm{~mL}$ fractions, pooled and concentrated to $12 \mathrm{mg} / \mathrm{mL}$ (Complex). Chromatogram of the export complex (Figure 3-5) was overlaid for comparison (B) $5 \mu \mathrm{L}$ of the fractions and $3 \mu \mathrm{g}$ of the "input" and the "complex" were analyzed by SDS-PAGE followed by Coomassie blue staining. (C) Full-length Xpo4 $(2 \mu \mathrm{g})$, which was used in the preparation of the complexes and for crystallization, was analyzed by SDS-PAGE and Coomassie blue staining.

After every new Xpo4 construct created for the import and export complexes, I used the same Xpo4 (also Xpo4-RanGTP) for crystallization trials. All the tested Xpo4 constructs throughout this study are summarized in Table 3-5.

\begin{tabular}{|lccccc|}
\hline Xpo4 & Solubility & \multicolumn{3}{c}{ Binding to } & \multirow{2}{*}{$\begin{array}{c}\text { Crystal } \\
\text { formation }\end{array}$} \\
\cline { 2 - 4 } & & RanGTP & elF5A & Sox2 & \\
\hline $1-460$ & ++ & Yes & No & No & \\
\hline $1-625$ & + & Yes & No & No & \\
\hline $1-691$ & ++ & Yes & No & Yes & Yes \\
\hline $1-759$ & + & Yes & No & Yes & \\
\hline$\Delta 241-260$ & ++++ & Yes & Yes & Yes & No \\
\hline$\Delta 931-948$ & ++++ & Yes & Yes & Yes & No \\
\hline$\Delta 241-260 \&$ & +++ & Yes & Yes & Yes & No \\
$931-948$ & & & & & \\
\hline$\Delta 559-600$ & - & & & & \\
\hline$\Delta 575-600$ & + & Yes & Yes & ? & \\
\hline$\Delta 654-668$ & ++++ & Yes & Yes & Yes & \\
\hline Full-length & ++++ & Yes & Yes & Yes & No \\
\hline
\end{tabular}

Table 3-5 Mapping of Xpo4 boundaries for ligand binding 
Xpo $4^{1-460}$ represents the $\mathrm{N}$-terminal fragment up to the acidic loop, Xpo $4^{\Delta 559-600}$ and $\mathrm{Xpo} 4^{\Delta 575-600}$ are the deletions of the insertion between HEATs 10 and 11, whereas Xpo4 ${ }^{\Delta 654-668}$ is that of the insertion between HEATs 11 and 12. Note that the second insertion was dispensable for Xpo4.

Although the crystallization trials for most of the constructs failed, biochemical analysis with elF5A, Sox2 and RanGTP revealed the essential parts of Xpo4 that were required for recognition of different interaction partners. In the end, similar to the import complex, promising crystallization conditions were obtained from Xpo4 $4^{1-691}$ and Xpo4 ${ }^{1-691}$-RanGTP complexes (Figure 3-41). 


\subsection{Exploring the Cargo Range of Xpo7}

As described in the introduction, Xpo7 is a member of the importin $\beta$ superfamily that mediates export of several structurally unrelated proteins and is therefore thought to have broad substrate specificity. Although, only three export cargoes have been characterized (Mingot et al., 2004; Dorfman and Macara, 2008), the work of our lab and the others have revealed that Xpo7 is required for the export of histones during erythroid maturation (Hattangadi et al., 2014), suggesting that the complete role of Xpo7 has not been fully elucidated. Therefore, we decided to investigate the function of Xpo7 by identifying the complete set of interaction partners of Xpo7.

\subsubsection{Identification of Xpo7 binders}

We decided to enrich Xpo7 interaction partners from a cellular extract via Xpo7 affinity chromatography. I designed and optimized an affinity chromatography method for reduced non-specific binding and therefore improved the possibility of identification of new interaction partners.

Bacterially expressed ED-SUMOvera-His12 tagged Xpo7 was immobilized to anti- $Z$ affibody beads. Then, these beads were incubated with a buffer or with a cytoplasmic HeLa cell extract in the absence or presence of RanGTP. After unbound proteins were washed away, His12-Xpo7 and bound proteins were eluted by incubating the beads with a SUMOvera protease. This step ensured that the binding that was specific to the matrix was eliminated. Next, the eluates were immobilized to a $\mathrm{Ni}$ (II) chelate matrix and Xpo7 binders were eluted with SDS. SDS can disrupt the interaction between His12-Xpo7 and the bound proteins but not the interaction between the matrix and His12; therefore, only bound proteins were recovered. Finally, the proteins were analyzed by SDS-PAGE. As shown in Figure 3-43, known Xpo7 interacting proteins were enriched in the presence of RanGTP. Surprisingly, several proteins bound to Xpo7 strongly only in the absence of RanGTP, and hence behaved like potential import cargoes. We identified some of these proteins by mass spectrometry as NAMPT, CutC, and the RBBP7 and HAT1 subunits of the type B histone acetyltransferase (HAT) complex (details will be discussed in the following sections). Nuclear localization of these proteins suggested that Xpo7 might import them to the nucleus. Overall, these 
results provided evidence for the presence of additional Xpo7 interaction partners that needed to be elucidated.

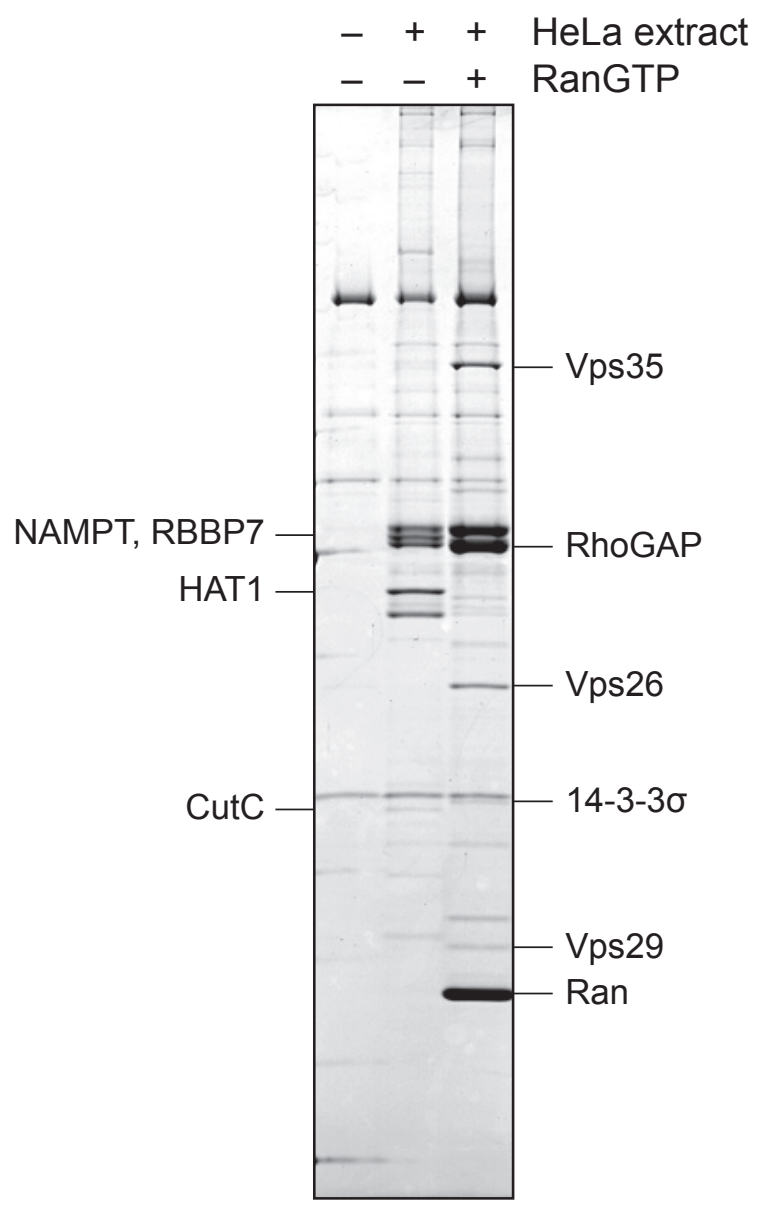

Figure 3-43 Xpo7 affinity chromatography with cytoplasmic HeLa extract

ED-SUMOvera-His12 tagged Xpo7 was immobilized to anti-Z affibody dimer coupled beads and incubated with a buffer (as a negative control) or with a cytoplasmic HeLa extract in the absence or presence of $5 \mu \mathrm{M}$ RanGTP. His 12-Xpo7 and bound proteins were recovered by protease elution and immobilized to $\mathrm{Ni}$ (II) chelate beads. Xpo7 bound proteins were eluted with SDS, analyzed by SDS-PAGE followed by Coomassie staining. Labeled bands were analyzed by mass spectrometry. ED is an affinity tag consisting $E$ and $D$ domains of protein A. SUMOvera is a protease cleavage site that is not recognized by eukaryotic SUMO protease.

\section{Discovery of novel binders via Xpo7 affinity chromatography}

So far, the identification of new interaction partners has depended on the visibility of a protein on the gel so that it can be excised and analyzed by mass spectrometry (MS). The result of the initial affinity chromatography suggested that Xpo7 might function as an importin in addition to its previously characterized role in nuclear export. This necessitates the enrichment of export cargoes and a release of import cargoes in the presence of RanGTP. For this reason, instead of analyzing the single bands, we decided to compare the protein levels in the 
sample containing RanGTP (+Ran) to the sample where RanGTP is absent (-Ran). Additionally, in order to increase the quantification sensitivity, and hence the identification efficiency, we decided to use the SILAC (stable isotope labeling by amino acids in cell culture) approach as a quantitative MS method (Ong et al., 2002).

SILAC relies on the use of two cell populations that are grown in identical conditions except the supplemented amino acids. In particular, one cell population is grown in a media containing natural amino acids (light), while the other one is in a media containing ${ }^{2} \mathrm{H},{ }^{13} \mathrm{C}$, or ${ }^{15} \mathrm{~N}$ labeled forms of the amino acids (heavy). When the light and the heavy extracts are analyzed on MS, they can be easily distinguished due to their mass shift. Therefore, the relative signal intensities are used to determine the protein abundances in different cell populations.

We obtained such heavy and light cytoplasmic HeLa extracts from Dr. Miroslav Nikolov from Mass Spectrometry Research Group, MPI-BPC and performed Xpo7 affinity chromatography in the absence or presence of $5 \mu \mathrm{M}$ RanGTP. As shown in Figure 3-44, both extracts performed similar in the affinity chromatography and gave identical results. Moreover, the results were similar to Figure 3-43.

Equal amounts of -Ran sample from the light extract and +Ran sample from the heavy extract (forward experiment), and +Ran sample from the light extract and -Ran sample from the heavy extract (reverse experiment) were mixed and analyzed by MS. The benefits of such an analysis are numerous. Firstly, as mentioned above, the relative intensity of a protein is easily determined by comparing light and heavy peptides. Secondly, errors that can be caused due to sample handling or instrumental processing are equalized for both samples. Thirdly, the forward and reverse experiments form experimental replicates, and therefore increases identification of false positives. MS analysis was performed by Samir Karaca from Mass Spectrometry Research Group, MPI-BPC. 


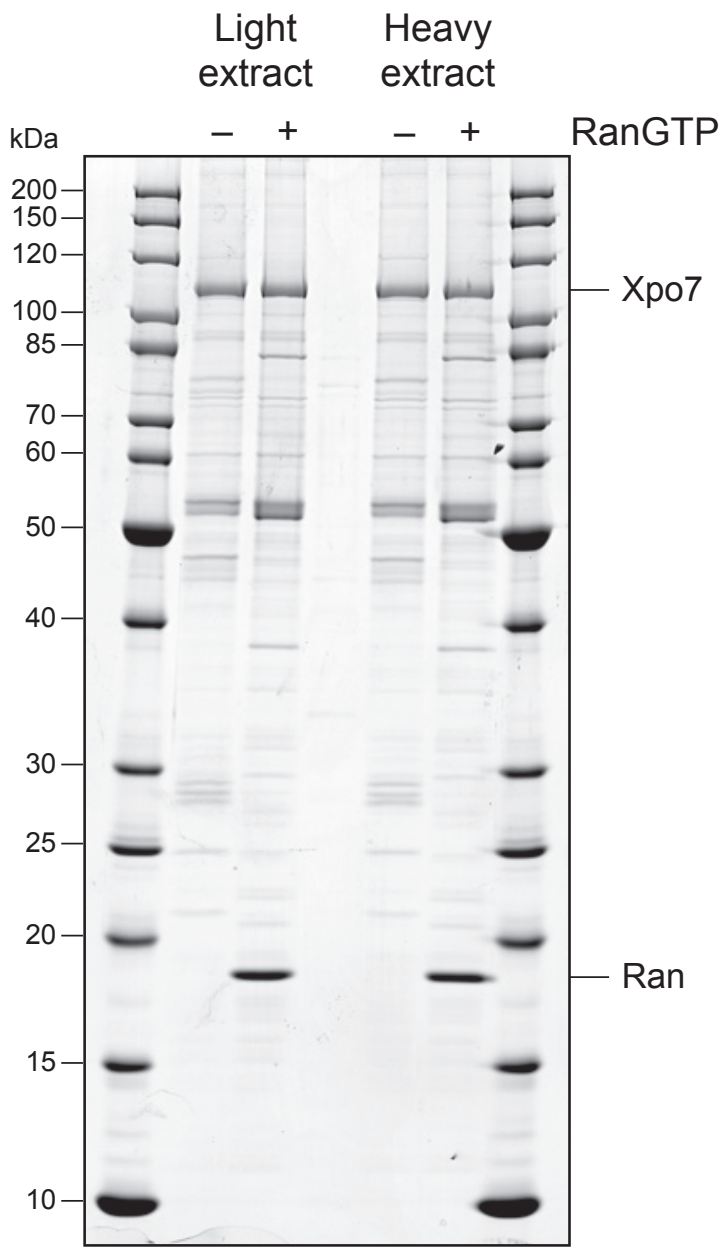

Figure 3-44 Xpo7 affinity chromatography using cytoplasmic HeLa extracts prepared with SILAC method

Affinity chromatography on immobilized Xpo7 was performed using cytoplasmic HeLa extracts labeled with light or heavy amino acids in the absence or presence of $5 \mu \mathrm{M}$ RanGTP. Bound proteins were analyzed by SDS-PAGE followed by Coomassie staining. Molecular weight markers were loaded left and right side of the gel and indicated accordingly on the left side.

\section{MS analysis of the eluates}

We analyzed the forward and reverse experiments together and identified approximately 750 proteins. The RanGTP enrichment level of each protein was calculated by dividing the signal intensity of the protein in +Ran sample to that of in -Ran sample, and the results were plotted as $\log _{2}$ values (Figure 3-45). Logarithmic depiction allowed us to observe the fold change in the relative protein amounts upon RanGTP addition. Positive values indicate RanGTP dependent binding, whereas negative values indicate RanGTP sensitive binding. In other words, the binding of a protein to Xpo7 is higher in the absence of RanGTP. 


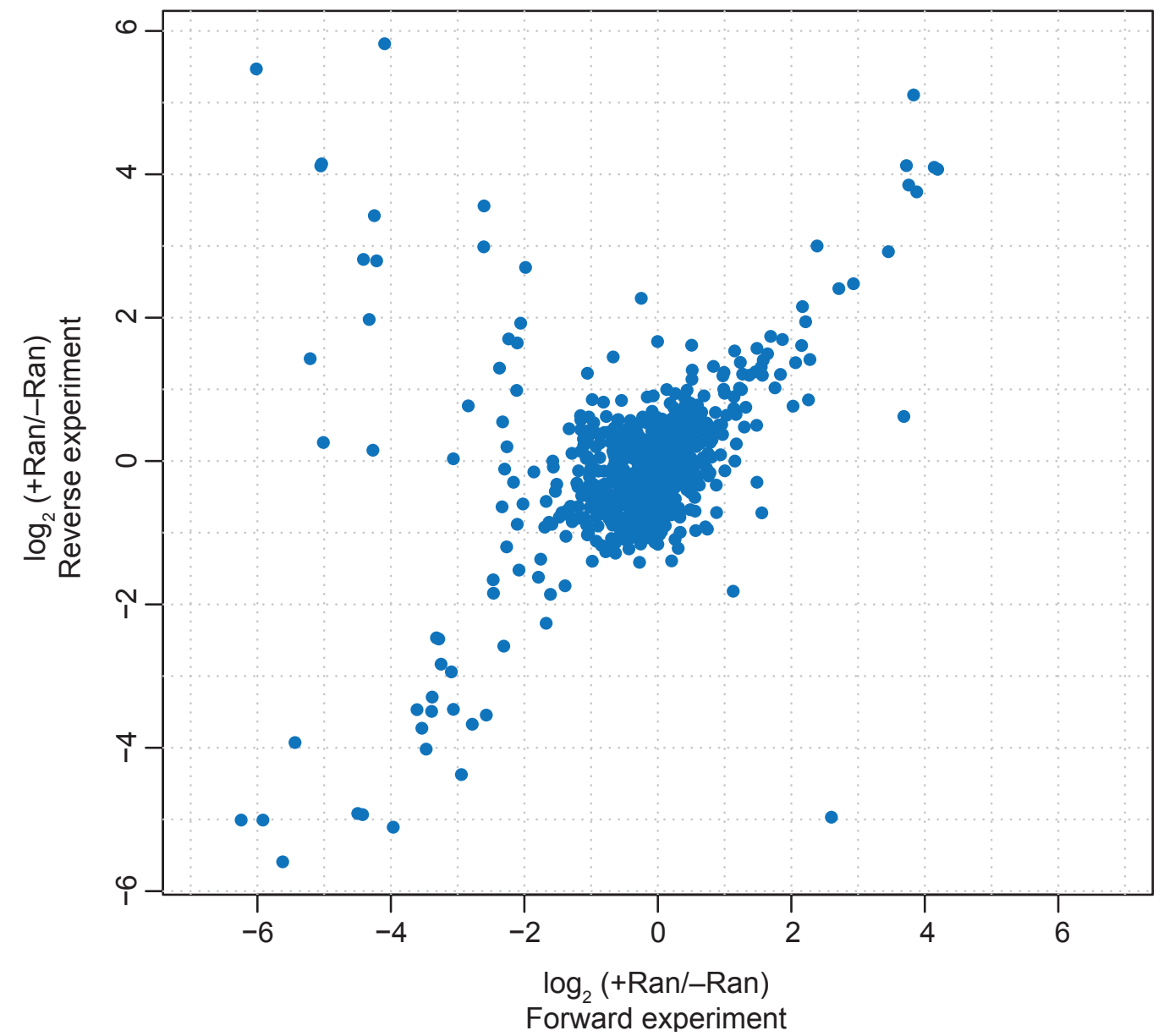

Figure 3-45 MS analysis of forward and reverse experiments

Ratios of protein levels obtained from forward (horizontal axis) and reverse (vertical axis) experiments are plotted as $\log _{2}$ values. The forward experiment contained -Ran sample from the light extract and + Ran sample from the heavy extract, whereas the reverse experiment contained -Ran sample from the heavy extract and +Ran from the light experiment

We classified the identified proteins into four groups (Figure 3-46). The first group was formed by the proteins whose level in the -Ran and +Ran samples was not significantly altered. This group consisted of $90 \%$ of the proteins (grey circles). These proteins were considered to be the background binders. The proteins that showed an inverse correlation with the presence of RanGTP in the two experiments formed the second group (dark grey circles). The protein level of most of the proteins in this group was higher in the light extract (in both experiments); therefore, these proteins were considered as light contaminants. The proteins that were supplied during the experiment like RanGTP and Xpo7 also belonged to this group. The third group consisted of the proteins that were enriched $\left(\log _{2}>1\right)$ in the presence of RanGTP in both experiments (green circles). This group contained potential export substrates and included all the previously characterized Xpo7 export cargoes as well as several other cytoplasmic proteins (Table 3-6). Finally, 
the proteins that showed RanGTP sensitive binding in both experiments formed the last group (red circles) and contained the potential import substrates. The group consisted of more than 20 proteins that had not been linked to Xpo7 so far (Table 3-7).

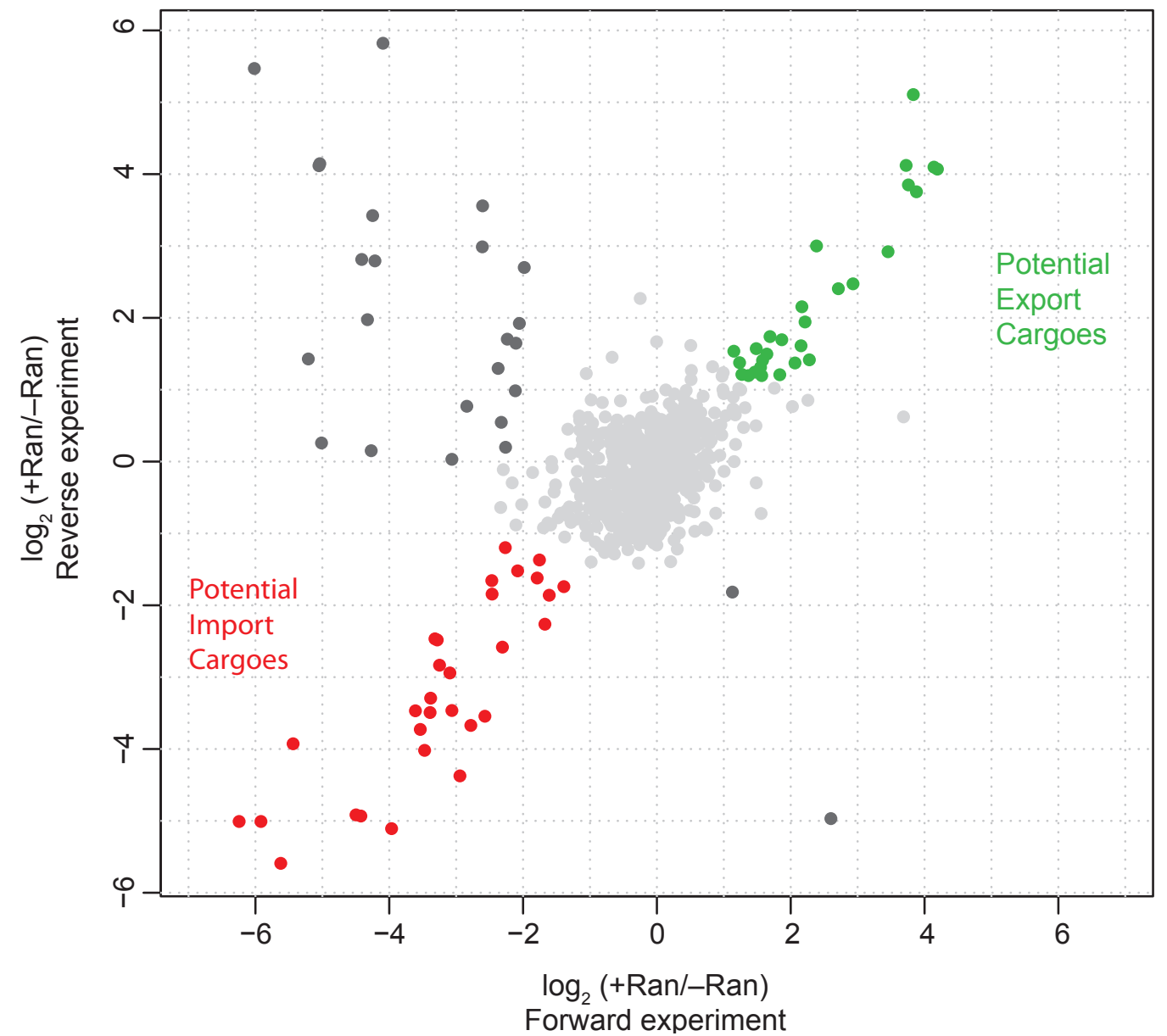

Figure 3-46 MS analysis of the SILAC experiments

Figure 3-45 is depicted with color-coding showing the four regions mentioned in the text. Green represents the proteins that are enriched in the presence of RanGTP in both experiments while red represents the proteins that bound Xpo7 in the absence of RanGTP. Non-specific binders are shown in grey and contaminants are in dark grey. 


\begin{tabular}{|c|c|c|c|c|}
\hline \multirow[b]{2}{*}{ RanGTP dependent Xpo7 binders } & \multirow[b]{2}{*}{ Gene } & \multirow{2}{*}{$\begin{array}{c}\mathrm{MW} \\
(\mathrm{kDa})\end{array}$} & \multicolumn{2}{|c|}{$\log _{2}(+\operatorname{Ran} /-\operatorname{Ran})$} \\
\hline & & & Forward & Reverse \\
\hline $\begin{array}{l}\text { Vacuolar protein sorting-associated protein } \\
26 \mathrm{~A}\end{array}$ & VPS26A & 38 & 4.193 & 4.069 \\
\hline Tetratricopeptide repeat protein $39 \mathrm{C}$ & TTC39C & 66 & 3.878 & 3.756 \\
\hline Vacuolar protein sorting-associated protein 35 & VPS35 & 92 & 3.833 & 5.105 \\
\hline 60 kDa SS-A/Ro ribonucleoprotein & TROVE2 & 61 & 3.761 & 3.849 \\
\hline Vacuolar protein sorting-associated protein 29 & VPS29 & 21 & 3.726 & 4.120 \\
\hline Rho GTPase-activating protein 1 & ARHGAP1 & 50 & 3.456 & 2.922 \\
\hline $\begin{array}{l}\text { Geranylgeranyl transferase type- } 2 \text { subunit } \\
\text { alpha }\end{array}$ & RABGGTA & 65 & 2.930 & 2.475 \\
\hline 14-3-3 protein sigma & SFN & 28 & 2.712 & 2.406 \\
\hline Tubulin-specific chaperone A & TBCA & 14 & 2.387 & 2.999 \\
\hline $\mathrm{PCl}$ domain-containing protein 2 & PCID2 & 46 & 2.279 & 1.417 \\
\hline TBC1 domain family member 5 & TBC1D5 & 91 & 2.214 & 1.942 \\
\hline $\begin{array}{l}\text { Vacuolar protein sorting-associated protein } \\
26 \mathrm{~B}\end{array}$ & VPS26B & 39 & 2.169 & 2.153 \\
\hline $60 S$ ribosomal protein $\mathrm{L} 7 \mathrm{a}$ & RPL7A & 30 & 2.154 & 1.610 \\
\hline GPN-loop GTPase 1 & GPN1 & 42 & 2.065 & 1.372 \\
\hline $60 S$ ribosomal protein L19 & RPL19 & 23 & 1.837 & 1.208 \\
\hline $\begin{array}{l}\text { Geranylgeranyl transferase type-2 subunit } \\
\text { beta }\end{array}$ & RABGGTB & 37 & 1.645 & 1.497 \\
\hline $60 S$ ribosomal protein $\mathrm{L} 13$ & RPL13 & 24 & 1.568 & 1.194 \\
\hline $60 S$ ribosomal protein L14 & RPL14 & 23 & 1.547 & 1.312 \\
\hline Peroxisomal targeting signal 1 receptor & PEX5 & 72 & 1.486 & 1.573 \\
\hline $40 S$ ribosomal protein S6 & RPS6 & 29 & 1.463 & 1.246 \\
\hline $\begin{array}{l}\text { Spliceosome RNA helicase DDX39B;ATP- } \\
\text { dependent RNA helicase DDX39A }\end{array}$ & $\begin{array}{l}\text { DDX39B; } \\
\text { DDX39A }\end{array}$ & 51 & 1.272 & 1.213 \\
\hline Nuclear pore complex protein Nup153 & NUP153 & 157 & 1.238 & 1.375 \\
\hline $\begin{array}{l}\text { Serine/threonine-protein phosphatase } 2 \mathrm{~A} 65 \\
\mathrm{kDa} \text { regulatory subunit A alpha isoform }\end{array}$ & PPP2R1A & 65 & 1.151 & 1.535 \\
\hline
\end{tabular}

Table 3-6 The list of RanGTP dependent Xpo7 cargoes

The proteins that that showed at least two fold enrichment in the presence of RanGTP are listed. Forward and reverse columns show the fold change $\left(\log _{2}(+\operatorname{Ran} /-\operatorname{Ran})\right.$ of proteins in the forward and reverse experiments, respectively. Previously characterized Xpo7 export cargoes are highlighted in light green. See Table 7-1 for the Uniprot identifiers. 


\begin{tabular}{|c|c|c|c|c|}
\hline \multirow[b]{2}{*}{ RanGTP sensitive Xpo7 binders } & \multirow[b]{2}{*}{ Gene } & \multirow{2}{*}{$\begin{array}{c}\text { MW } \\
(\mathrm{kDa})\end{array}$} & \multicolumn{2}{|c|}{$\log _{2}(+\operatorname{Ran} /-\operatorname{Ran})$} \\
\hline & & & Forward & Reverse \\
\hline $\begin{array}{l}\text { Single-strand selective monofunctional } \\
\text { uracil DNA glycosylase }\end{array}$ & SMUG1 & 30 & -6.242 & -5.007 \\
\hline Histone-binding protein RBBP7 & RBBP7 & 48 & -5.915 & -5.010 \\
\hline $\begin{array}{l}\text { Guanosine-3,5-bis(diphosphate) 3- } \\
\text { pyrophosphohydrolase MESH1 }\end{array}$ & HDDC3 & 20 & -5.623 & -5.593 \\
\hline Ran-binding protein 1 & RANBP1 & 23 & -5.437 & -3.926 \\
\hline Copper homeostasis protein cutC homolog & CUTC & 29 & -4.496 & -4.915 \\
\hline Porphobilinogen deaminase & HMBS & 39 & -4.423 & -4.933 \\
\hline $\begin{array}{l}\text { Histone acetyltransferase type B catalytic } \\
\text { subunit }\end{array}$ & HAT1 & 50 & -3.963 & -5.109 \\
\hline $\begin{array}{l}\text { BTB/POZ domain-containing protein } \\
\text { KCTD15 }\end{array}$ & KCTD15 & 32 & -3.608 & -3.468 \\
\hline $\begin{array}{l}\text { Bifunctional protein NCOAT;Protein O- } \\
\text { GIcNAcase;Histone acetyltransferase }\end{array}$ & MGEA5 & 103 & -3.537 & -3.728 \\
\hline Histone deacetylase 8 & HDAC8 & 42 & -3.472 & -4.018 \\
\hline Ribonuclease $\mathrm{P}$ protein subunit $\mathrm{p} 40$ & RPP40 & 42 & -3.391 & -3.493 \\
\hline THO complex subunit 6 homolog & THOC6 & 38 & -3.379 & -3.294 \\
\hline GMP synthase & GMPS & 77 & -3.316 & -2.467 \\
\hline $\begin{array}{l}\text { Arf-GAP with Rho-GAP domain, ANK repeat } \\
\text { and PH domain-containing protein } 3\end{array}$ & ARAP3 & 170 & -3.280 & -2.483 \\
\hline Selenocysteine lyase & SCLY & 48 & -3.250 & -2.832 \\
\hline $\begin{array}{l}\text { ATP-dependent RNA helicase DDX3X; } \\
\text { ATP-dependent RNA helicase DDX3Y }\end{array}$ & $\begin{array}{l}\text { DDX3X; } \\
\text { DDX3Y }\end{array}$ & 73 & -3.093 & -2.940 \\
\hline $\begin{array}{l}\text { Queuine tRNA-ribosyltransferase subunit } \\
\text { QTRTD1 }\end{array}$ & QTRTD1 & 47 & -3.062 & -3.465 \\
\hline Queuine tRNA-ribosyltransferase & QTRT1 & 44 & -2.943 & -4.374 \\
\hline Putative nuclease HARBI1 & HARBI1 & 39 & -2.779 & -3.670 \\
\hline Nicotinamide phosphoribosyltransferase & NAMPT & 56 & -2.572 & -3.543 \\
\hline Ran GTPase-activating protein 1 & RANGAP1 & 64 & -2.468 & -1.658 \\
\hline Nuclear autoantigenic sperm protein & NASP & 86 & -2.461 & -1.843 \\
\hline LanC-like protein 1 & LANCL1 & 45 & -2.310 & -2.583 \\
\hline Exportin-2 & CSE1L & 110 & -1.787 & -1.621 \\
\hline
\end{tabular}

Table 3-7 The list of potential Xpo7 import substrates

The proteins that that showed at least two fold enrichment in the absence of RanGTP are listed. Forward and reverse columns show the fold change $\left(\log _{2}(+\operatorname{Ran} /-\operatorname{Ran})\right.$ of proteins in the forward and reverse experiments, respectively.. See Table $7-2$ for Uniprot identifiers.

\subsubsection{Validation of the interaction of the selected binders with Xpo7}

So far, we identified a new set of proteins that interacted with Xpo7 in a RanGTP dependent or RanGTP sensitive manner. The latter suggests that Xpo7 might function as an importin, supporting our initial findings with Xpo7 affinity 
chromatography. Although some of the RanGTP sensitive binders were cytoplasmic proteins, most of them were nuclear; therefore, the import of these proteins by Xpo7 was an attractive possibility. In order to validate the binding of these candidates to Xpo7, I selected nine candidates and performed pull-downs and binding assays. Moreover, I included a candidate from the potential export cargo list to these assays. A short summary of the selected candidates will follow:

Single-strand selective monofunctional uracil DNA glycosylase (SMUG1) is a glycosylase that removes uracil from preferentially single-stranded DNA and therefore functions in base excision DNA repair (Haushalter et al., 1999; Masaoka et al., 2003). Histone-binding protein RBBP7 (RBBP7) and histone acetyltransferase type B catalytic subunit (HAT1) are the subunits of the type $B$ histone acetyltransferase (HAT-B) complex. HAT-B complex acetylates soluble H4 and is though to play a role in nucleosome assembly (Verreault et al., 1998; Makowski et al., 2001). Guanosine-3,5-bis(diphosphate) 3-pyrophosphohydrolase MESH1 (MESH1) is a cytoplasmic protein that functions in starvation response (Sun et al., 2010). Copper homeostasis protein cutC homolog (CutC) can bind to $\mathrm{Cu}^{1+}$ and may function in copper homeostasis (Li et al., 2005). Porphobilinogen deaminase (HMBS) is another cytoplasmic protein of the import cargo candidates and is involved in the heme biosynthesis by catalyzing the head to tail condensation of four porphobilinogen molecules into hydroxymethylbilane (UniProt, 2015). Protein O-GlcNAcase (MGEA5) cleaves $\mathrm{N}$-acetylglucosamine from O-glycosylated proteins and can function both in the nucleus and cytoplasm (Gao et al., 2001). Histone deacetylase 8 (HDAC8) is a member of the class I histone deacetylases and plays a role in transcriptional regulation (Buggy et al., 2000; Hu et al., 2000; Van den Wyngaert et al., 2000). Nicotinamide phosphoribosyltransferase (NAMPT) is involved in the NAD biosynthesis and is responsible for the production of nicotinamide mononucleotide (Rongvaux et al., 2002). It might also function as adipokine in the bloodstream (Romacho et al., 2013). Finally, alpha and beta subunits (RABGGTA and RABGGTB) of the type II geranylgeranyl transferase are the only tested export candidates. This enzyme transfers the geranylgeranyl moiety to various Rab proteins and directs them to the corresponding membrane (Farnsworth et al., 1994). 

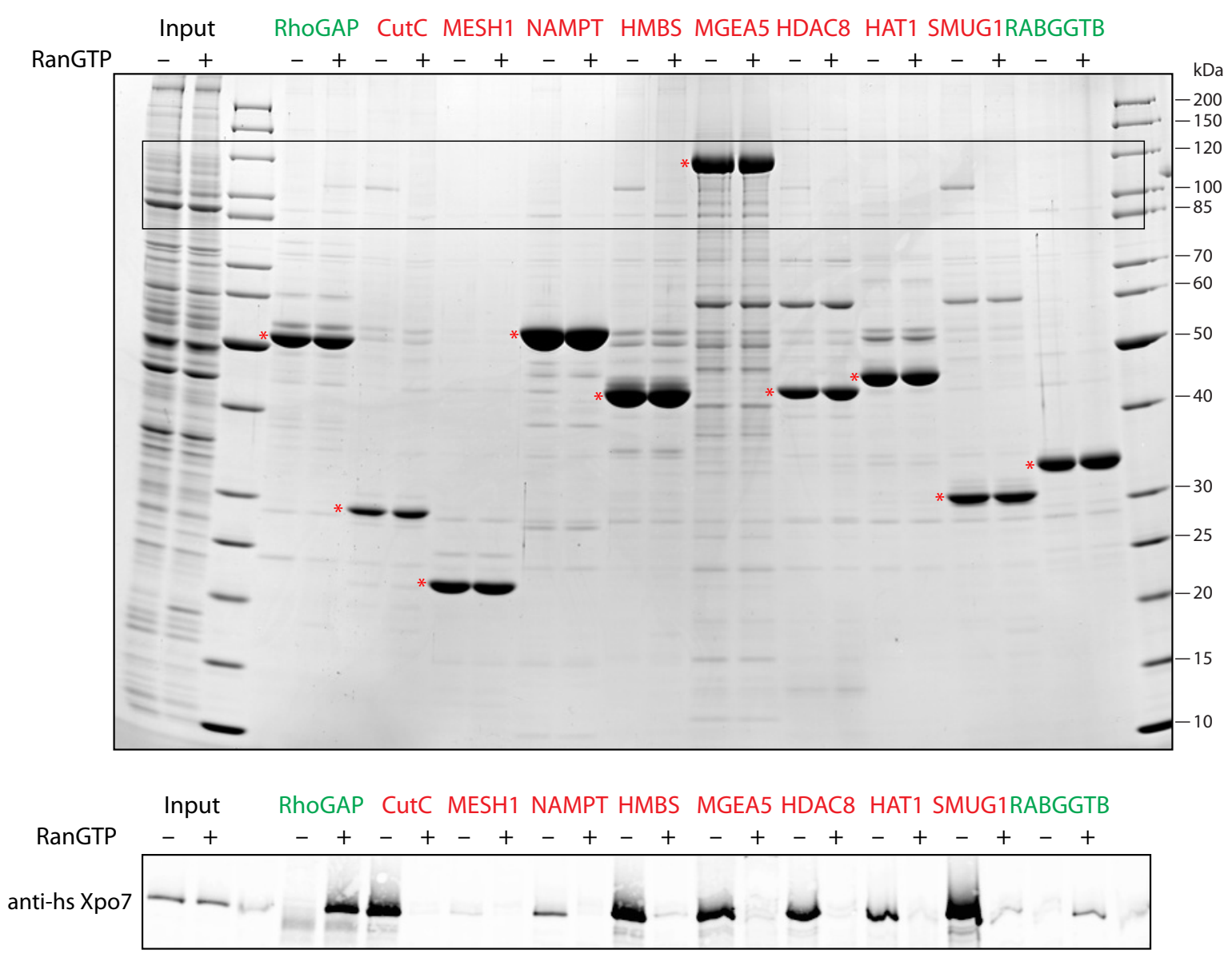

Figure 3-47 Identified candidate proteins enrich Xpo7 from a cytoplasmic extract in a RanGTP regulated manner

0.5 pmol H14-ZZ-Nedd8 tagged candidates were immobilized to Z-affibody dimer beads. Then, the beads were incubated with $250 \mu \mathrm{L}$ cytoplasmic HeLa extract either supplemented with $5 \mu \mathrm{M}$ RanGTP or same volume of buffer. After $3 \mathrm{~h}$ incubation, unbound proteins were removed; the immobilized cargoes and bound proteins were eluted by incubating the beads with $250 \mathrm{nM}$ bdNEDD8 protease. Both input and eluates were separated by SDS-PAGE. One gel was analyzed by Coomassie staining (upper panel). The other one was analyzed by western blotting with anti-hsXpo7 antibody (lower panel). The area corresponding to the highlighted area in the upper panel is shown in the lower panel. Potential export and import cargoes are colored in green and red, respectively. Red asterisks mark the corresponding cargoes.

I cloned these candidates into a vector containing His14-ZZ-NEDD8 tag. I also cloned RhoGAP into a similar construct to use as a positive control for Xpo7 binding. All of these constructs were expressed in E. coli. RBBP7 and RabGGTA were not soluble; therefore I did not include these proteins in the further experiments. To validate the specificity of Xpo7 binding, I immobilized these proteins to anti-Z affibody beads and then incubated with a cytoplasmic HeLa extract either in the absence or presence of $5 \mu \mathrm{M}$ RanGTP. After the unbound proteins were removed and the beads were washed, the immobilized candidates and bound proteins were recovered by incubating the beads with bdNEDD8 protease. The eluates were first separated by SDS-PAGE and then analyzed either by Coomassie-staining or by western blotting using an anti-humanXpo7 
antibody (Figure 3-47). All of the tested proteins enriched Xpo7 from the extract, indicating that these proteins in fact interact with Xpo7. RhoGAP and RabGGTB enriched Xpo7 only in the presence of RanGTP and therefore behaved like export cargoes. In contrast, in the other proteins, Xpo7 enrichment significantly reduced in the presence of RanGTP, which was a characteristic of the import substrates. It should be noted that the Xpo7 binding in MESH1, NAMPT and RabGGTB was relatively weaker compared to the other proteins.

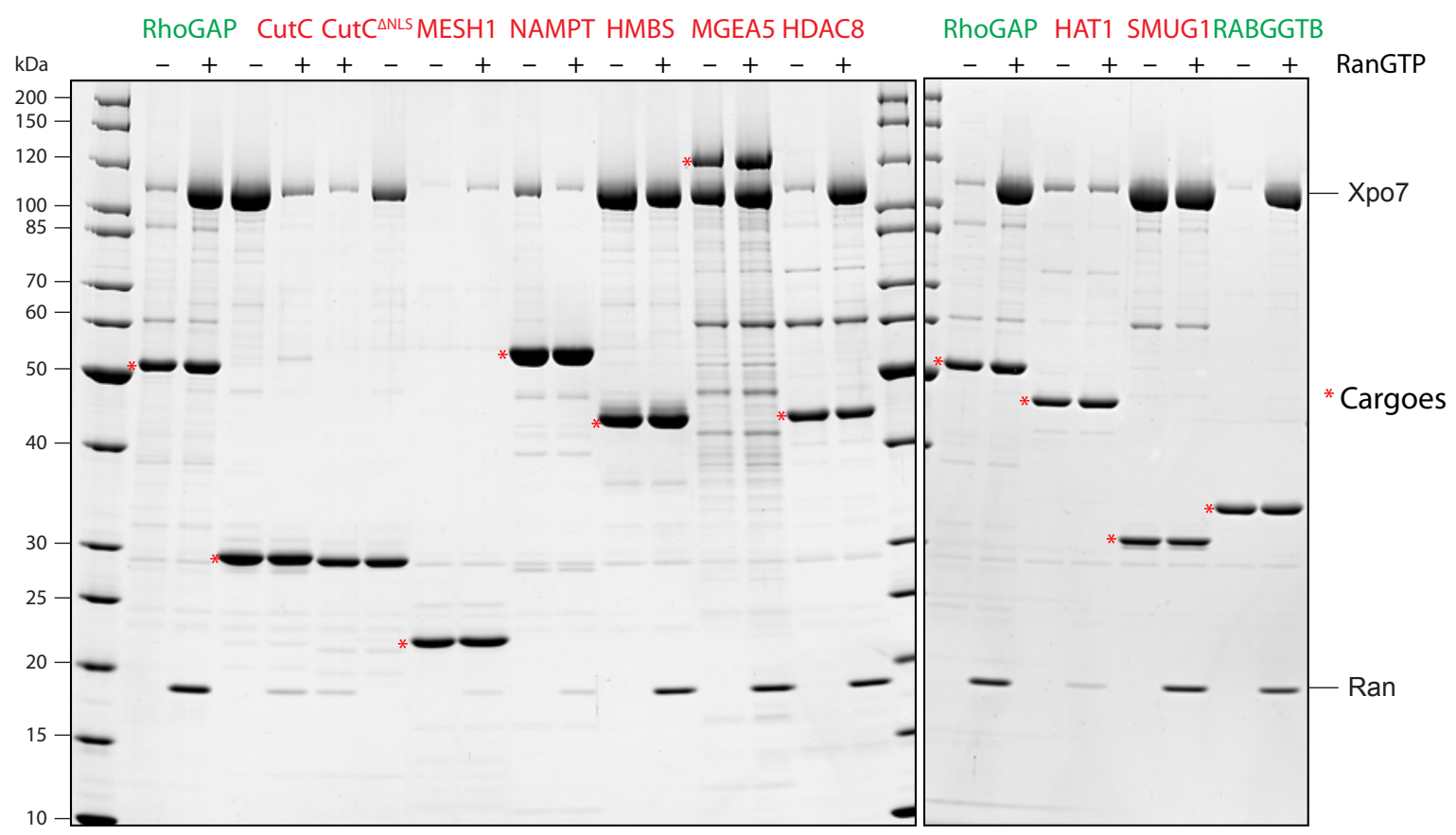

Figure 3-48 Binding assay with candidates and recombinant Xpo7

$0.5 \mu \mathrm{M} \mathrm{H} 14-\mathrm{ZZ}-N E D D 8$ tagged cargoes were incubated with $0.75 \mathrm{Xpo} 4$ in the absence or presence of $1.5 \mu \mathrm{M}$ RanGTP in $500 \mu \mathrm{L}$ at $50 \mathrm{mM} \mathrm{NaCl}$. After $2 \mathrm{~h}$ incubation at $4{ }^{\circ} \mathrm{C}$ the samples were incubated with Z-affibody dimer beads to capture the cargoes and bound proteins. After another $2 \mathrm{~h}$ incubation, unbound proteins were removed, RanGTP and bound proteins were eluted by incubating the beads with $250 \mathrm{nM}$ bdNEDD8 protease. Eluates were separated by SDS-PAGE and analyzed by Coomassie staining. Potential export and import cargoes are colored in green and red, respectively. Red asterisks mark the corresponding cargoes. Cargoes on the left and on the right were tested on different dates, as a control RhoGAP was included in both panels. Similar experiment at $100 \mathrm{mM} \mathrm{NaCl}$ gave identical results. CutC ${ }^{\triangle \mathrm{NLS}}$ represents the construct where the classical nuclear localization signal (RKRAR) on CutC was mutated to TGSAT to test if the interaction depends on NLS.

We have found that all of the selected candidates interact with Xpo7, however, this interaction could have happened directly or via an adaptor molecule. Therefore I wanted to test the direct interaction with recombinantly expressed Xpo7. I followed the same experimental procedure described above. Instead of a cytoplasmic extract, I used recombinant Xpo7 and tested the Xpo7 binding of these cargoes in the absence and presence of RanGTP. Figure 3-48 shows the SDS-PAGE analysis of the experiment. Xpo7 binding, although very weak for some proteins, was seen in all proteins, indicating that these proteins contain contact sites for 
Xpo7. RhoGAP and RabGGTB bound to Xpo7 only in the presence of RanGTP. Additionally, the potential import cargo HDAC8 also bound to Xpo7 only in the presence of RanGTP, and hence behaved like an export cargo in the isolated context. Moreover, the Xpo7 binding of HMBS, MGEA5 and SMUG1 was also different than the previous experiments. These proteins bound to Xpo7 in a RanGTP independent manner. In other words, RanGTP presence did not affect Xpo7 binding to these proteins in this given experimental setting. Xpo7 binding to CutC and NAMPT was as expected and took place only in the absence of RanGTP. Finally, MESH1 and HAT1 also bound to Xpo7, however, the bound Xpo7 was fairly low.

\subsubsection{Does Xpo7 form a dimer?}

In the last year of my PhD work, I supervised a lab rotation student, Matthew G. Logsdon, who investigated the putative import activity of Xpo7. Although his work is not part of this thesis, I will describe one important discovery.

While characterizing an import cargo-Xpo7 complex, we analyzed Xpo7 on MALS and found out that the predicted mass of the molecule was $\sim 255 \mathrm{kDa}$ (Figure 3-49), which suggested a homodimer. The observation was verified with different preparations and with different buffer conditions. Although we did not further probe the nature of the dimer, I will discuss structural and functional implications of the dimer in the second part of the following section.

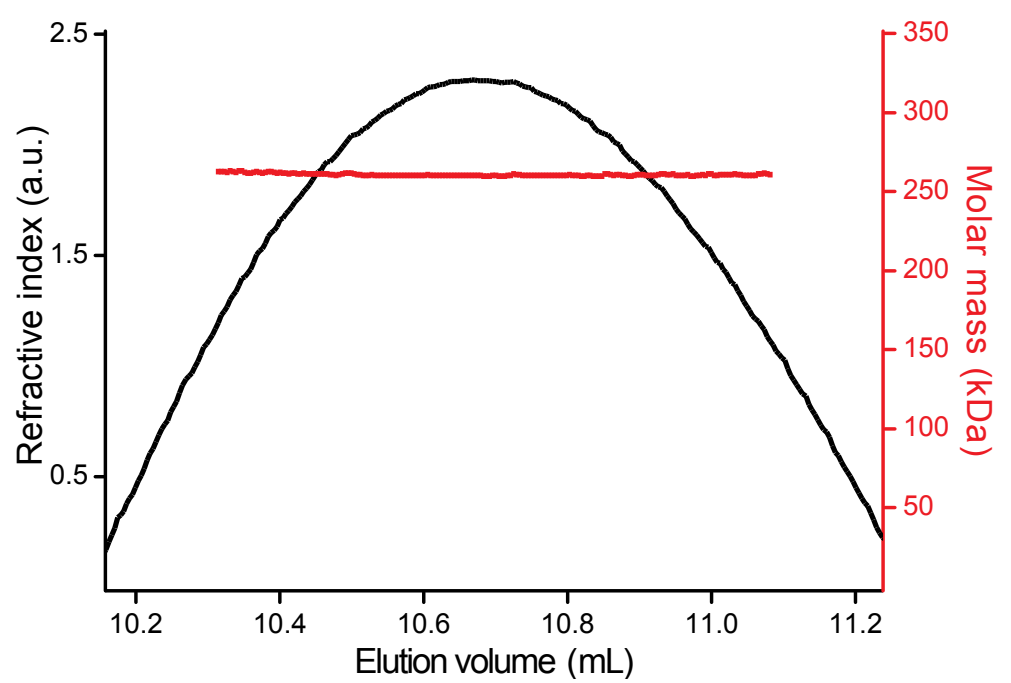

Figure 3-49 MALS analysis of Xpo7

Gel filtration purified Xpo7 was concentrated to $2 \mathrm{mg} / \mathrm{ml}$ and analyzed by Superdex 200 10/30 column coupled to MALS detector. Theoretical molar mass of Xpo7 construct was $125 \mathrm{kDa}$, whereas experimental mass was $255 \mathrm{kDa}$, suggesting a dimer. 


\section{DISCUSSION}

\subsection{Xpo4 mediated nucleocytoplasmic transport}

Structural studies are crucial elements for the comprehensive understanding of NTR function at the molecular level (Cook and Conti, 2010). NTRs are built from the same architectural units (HEAT repeats) and yet adopt different tertiary structures. Even the same NTR can adopt different conformations in its cargo-bound and unbound states. Therefore, the three-dimensional architecture of an NTR cannot be determined without structural information. NTRs functionalize their HEAT repeats in many different forms for cargo (and RanGTP) interactions. First of all, importins and exportins respond to RanGTP-binding either with cargo loading or unloading. Besides this very characteristic difference, NTRs also have their unusual members. Some proteins are cargoes of both an exportin and an importin, shuttling continuously. On the other hand, a cargo might be ferried to the same destination by more than one NTR to facilitate the transport. Alternatively, the same NTR can be the exportin for some proteins and also an importin for others. Given all these extraordinary cases, it is impossible for us to foresee the molecular mechanism by which the NTRs interact with their cargoes without structural information.

Xpo4 is a bidirectional NTR that exports elF5A and Smad3 and imports Sox2 and SRY. Xpo4 has been recently identified as a tumor-suppressor protein (Zender et al., 2008). Having both transcription and translation factors as its cargoes Xpo4 might act as a tumor-suppressor by confining their localization and therefore their activity to certain compartments. This would also give considerable medical interest to Xpo4-mediated nucleocytoplasmic transport. Although structures of several importins and exportins have been elucidated before, there has been no structural information available for Xpo4 and it has been unclear how Xpo4 mediates transport of distinct cargoes in opposite directions. In my PhD work, we have elucidated the structure of the elF5A nuclear export complex. The structure of the complex and the biochemical data presented in section 3.1 provide insights into how Xpo4 functions as a bidirectional NTR. 


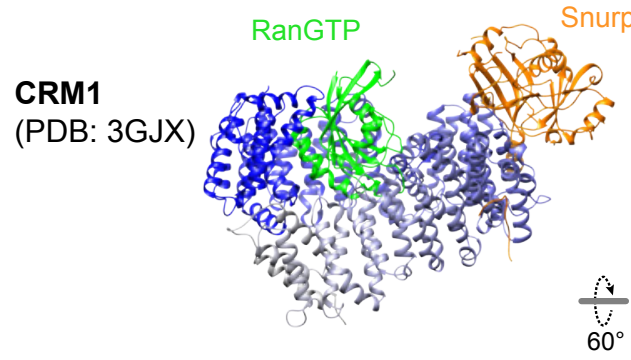

\section{Snurportin 1}

CAS

(PDB: 1WA5)
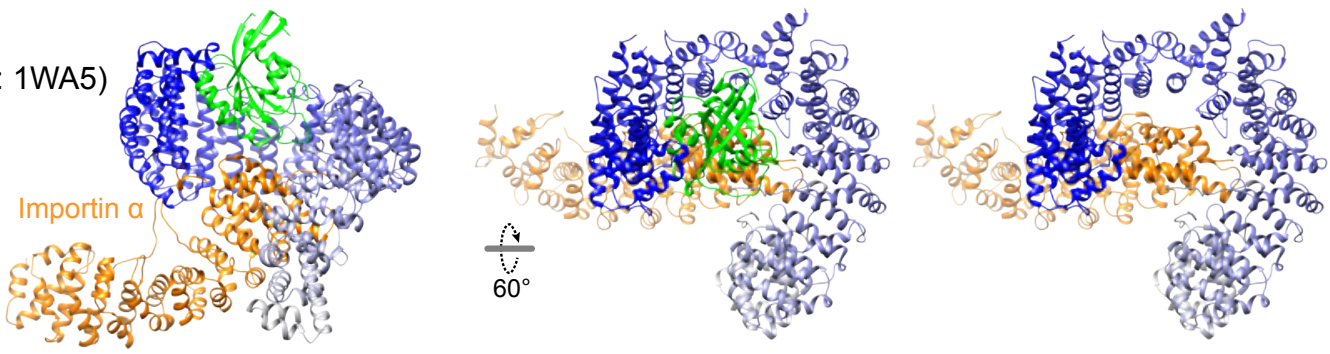

Xpot

(PDB: 3ICQ)
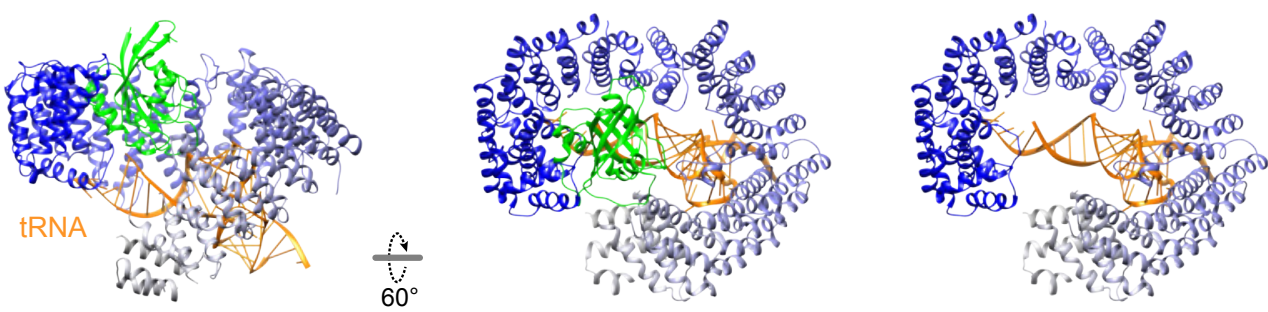

Xpo4

(This study)
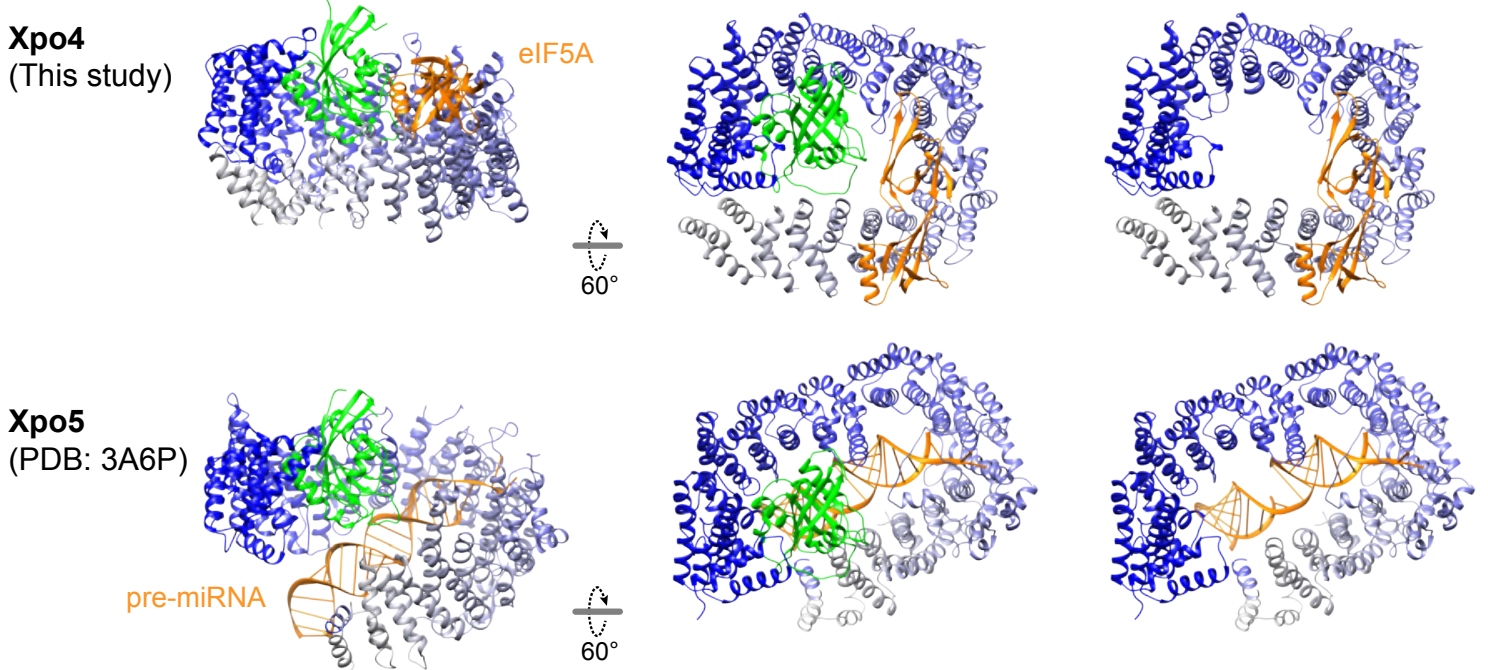

Ipo13

(PDB: 3ZJY)
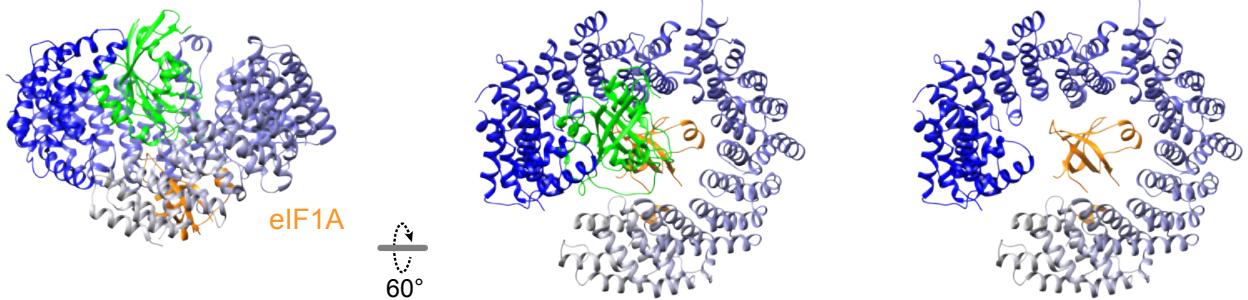

Figure 4-1 Comparison of cargo binding by exportins

All export complexes are aligned with respect to RanGTP and illustrated in a ribbon representation. Exportins are depicted as a color gradient from blue ( $\mathrm{N}$-terminus) to grey (C-terminus). The respective cargoes are shown in orange and Ran is in green. Left and middle: Export complexes are visualized in two orientations. Right: Complexes are displayed as in the middle, but Ran was removed. 
The architecture of Xpo4, despite the sequence variations and relatively larger size (for instance, Xpo4 is $129 \mathrm{kDa}$ and Imp13 is $108 \mathrm{kDa}$; the similarity is $15 \%$ ), is similar to the other exportins and made up of 20 HEAT repeats. Larger size is due to the intra-repeat and inter-repeat insertions that bent the overall structure into a toroid like shape. Ran is enclosed by this toroid, similar to that observed in CRM1Ran interaction, leaving very small area inside the toroid for elF5A. Xpo4 does not recognize a linear export signal but folded domains of elF5A. Notably, elF5A is recognized by Xpo4 in an unprecedented manner. elF5A is placed over the toroid on a cavity created by the intra-repeat loops of HEATs 11 to 16 , while the basic hypusine-containing loop is buried in an acidic groove. Interactions at the acidic groove are indispensible for elF5A binding as well as the export activity of Xpo4.

The binding mode of Xpo4 to its export cargo is different than that observed in other exportins (Figure 4-1). In the nuclear export complex structures solved to date, the exportins CAS, Xpot, Xpo5 and Ipo13 coil around the cargoes, while the basic patch of Ran interacts with acidic residues of the cargoes (Matsuura and Stewart, 2004; Cook et al., 2009; Okada et al., 2009; Grunwald et al., 2013). CRM1, on the other hand, recruits the cargo to the outer surface of the toroid and there is no direct contact between the cargo and Ran (Monecke et al., 2009). Xpo4 neither coils around nor recruits the cargo to the outer surface; instead, elF5A is positioned above the toroid. Moreover, elF5A interacts with Ran through a single glutamic acid residue (elF5A Glu42), which is not essential for elF5A binding (Figure 3-34).

The overall shapes of Xpo4, Imp13 and CRM1 in their cargo-bound form are almost indistinguishable and yet all of them bind their cargoes differently (Figure 4-1). What makes these NTRs, or specifically Xpo4 special? The answer is in the details. Both, Xpo4 and Imp13 recognize their cargoes by charge and shape complementarity. Although the cargoes are acidic translation factors, these exportins interact mostly with basic residues of these proteins. Xpo4 and Imp13 are highly acidic proteins. However, the acidic residues are distributed differently in these NTRs such that they interact with their cargoes through different regions.

At the sequence level, CRM1 is the second closest relative of Xpo4 among the Imp $\beta$ family (after Xpo7). The size and shape of the individual HEAT repeats of Xpo4 and CRM1 are also very similar. However, three distinct features of these 
NTRs lead to different cargo recognition modes. First, similar to $\operatorname{Imp} 13$, the charge distribution of CRM1 is different. Second, the acidic loop of CRM1 is much longer than that of Xpo4. It would clash with elF5A; therefore, CRM1 would not be able to bind elF5A through the acidic loop. Third, the topology around the nuclear export signal (NES) binding site is different in Xpo4. NESs bind the hydrophobic cleft of CRM1 created by the HEATs 11 and 12. Corresponding region of Xpo4 is occupied by an NES-like fragment in the long insertion between HEATs 10 and 11 (see Figure 3-22 and the text in page 61). This makes Xpo4 incompatible for NES binding. Since Xpo4 emerged later in the evolution, the sequence and structural similarity might suggest that Xpo4 was evolved from CRM1 through gene duplication. In such a situation, blocking the NES-binding site by an NES-like insertion would be an elegant mechanism on the way to acquire a new function.

\subsubsection{Implications for cargo loading and cargo release mechanisms}

RanGTP is essential for export complexes. It increases the affinity of exportin to its cargo $\geq 1000$ fold. Ran mediates this either by causing conformational changes in the exportin, hence activating the cargo-binding site or by directly interacting with the cargo (Güttler and Görlich, 2011). All so far crystallized exportins employ both mechanisms at the same time, the exception being CRM1 where Ran does not contact the cargo. How does Ran promote cargo loading onto Xpo4? In the elF5A export complex, the interaction interface between Ran and the cargo is very small compared to that in the other export complex structures and this interaction is dispensable. Consequently, export complex assembly is likely to be triggered by Ran inducing conformational changes in Xpo4. Although the details require the structure of unliganded (or import cargo-bound) Xpo4, it is tempting to speculate that the acidic loop of Xpo4 might orchestrate a point of communication between RanGTP binding and elF5A loading. Considering the fact that the $\mathrm{N}$-terminal positioning of elF5A is crucial for complex formation, one can assume that the binding of Ran stabilizes the possibly flexible acidic loop and provides initial contact site for elF5A. Following conformational changes in the C-terminal of Xpo4 would complete complex assembly. Alternatively, Ran-binding can lock the already positioned eIF5A on the toroid and increase the affinity of exportin to its cargo by decreasing the dissociation rate of the cargo. 
Although the canonical cargo-free exportins exhibit low affinity for RanGTP, bidirectional NTRs like Xpo4 and Imp13 bind RanGTP rather strongly. Therefore, elF5A possibly will also encounter Xpo4•RanGTP complex in the nucleus. In the export complex, the hypusine-containing loop is locked in the acidic pocket by the acidic loop and HEAT repeats of Xpo4. As a result, the insertion of the hypusinecontaining loop into the acidic pocket is sterically not possible. This suggests that Xpo4•RanGTP complex should have an open conformation compared to the presented export complex. Indeed, we have obtained supportive information from mutational analysis of Xpo4 (Figure 3-31 and Figure 3-32).

When an export complex reaches to the cytoplasm, it disassembles upon stimulation of the GTPase activity of Ran, releasing the cargo to the cytoplasm. In the elF5A export complex, the interaction site of RanGAP on Ran is protected by the C-terminal HEAT repeats of Xpo4, and thus is inaccessible (Figure 4-2 and Lipowsky et al., 2000). It is likely that RanGTP is separated from the export complex by RanBP1 (or RanBP2) prior to GTP hydrolysis. Therefore, the mechanism of elF5A export complex disassembly seems to be similar to that observed in other export complexes. When the RanGTP.RanBP1 complex is superimposed to the elF5A export complex, RanBP1 and the C-terminal acidic tail of Ran would clash with intra-repeat loops of Xpo4 (Figure 4-2). In addition, RanBP1 would severely clash with N-terminal domain of elF5A. As a result, RanBP1-binding would destabilize the export complex and trigger elF5A release.
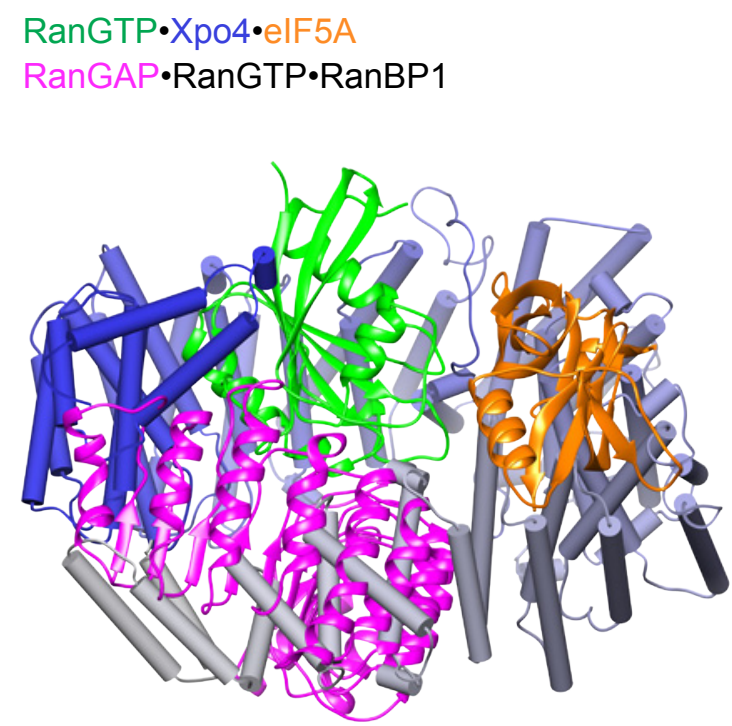

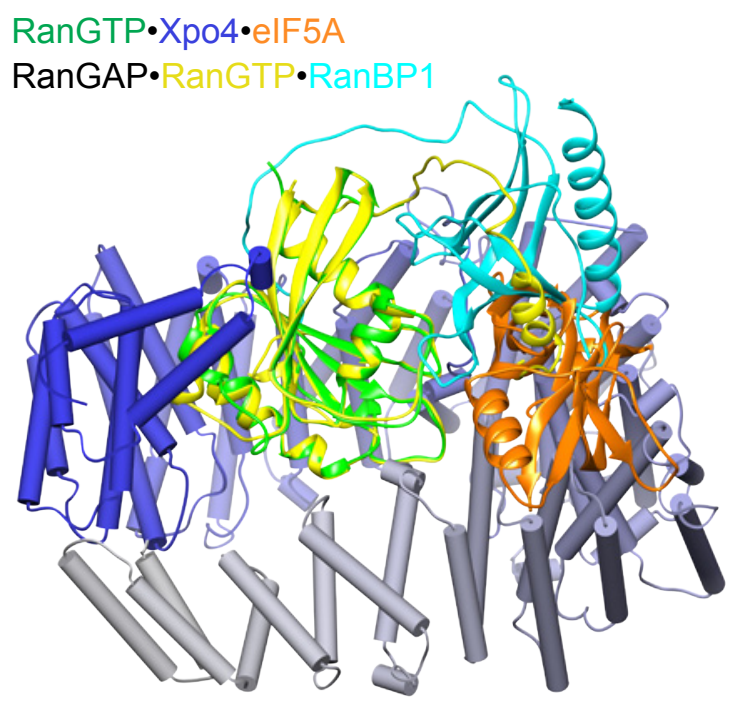

Figure 4-2 Cytoplasmic disassembly of the export complex 
RanGTP•Xpo4•elF5A complex is overlaid with RanGAP•RanGTP•RanBP1 structure (PDB ID 1K5D; Seewald et al., 2002). The structures are aligned via RanGTP. elF5A export complex is shown in a similar color-coding and orientation as in Figure 3-21. On the left, RanGAP (magenta) is shown in a ribbon representation. On the right, RanGTP and RanBP1 are shown in yellow and cyan, respectively. Note the severe clashes of RanGAP with C-terminal of Xpo4 and RanGTP.RanBP1 with Xpo4 and elF5A. (Adapted from Aksu et al., 2016)

\subsubsection{Implications for Xpo4 function}

Imp $\beta$-like NTRs are characterized primarily by their ability to carry cargoes across the nuclear envelope. In addition, they have another very essential function: they act as chaperones for their cargoes. This function is well described for importins, which recognize exposed basic patches of their cargoes as nuclear localization signals. Most of the proteins destined for the nucleus, such as histones and ribosomal proteins, have DNA- or RNA-binding function and contain highly basic domains. By binding to these domains, importins block interaction of these proteins with polyanionic substances (such as tRNAs or acidic proteins) in the cytoplasm and therefore prevent cytoplasmic aggregation of these proteins (Jakel et al., 2002). Same function is also seen in exportins. In the Xpo5 export complex, pre-miRNA is surrounded by Xpo5 and RanGTP, and protected from any endonuclease activity (Okada et al., 2009). Consequently, NTRs shelter their cargoes against unwanted interactions before and during the transport (Jakel et al., 2002).

Hypusination of eIF5A is essential for cell viability and is required for binding to RNAs, ribosomes and possibly to the other interaction partners and therefore critical component of elF5A function (Park et al., 2010). In the elF5A export complex, hypusine docks into an acidic groove of Xpo4 and is shielded from the environment. Consequently, Xpo4 would prevent any interaction partner approaching elF5A. In fact, in the absence of Xpo4, elF5A accumulates in nucleoli probably interacting with newly assembled ribosomes and disengages upon Xpo4 addition (Figure 3-36 and Lipowsky et al., 2000). Therefore, Xpo4 acts like an elF5A inhibitor in the nucleus and during the transport. It should be noted that while stoichiometric amounts of Xpo4 would be needed to block elF5A function in the nucleus, by exporting elF5A from the cytoplasm, Xpo4 accomplishes the same function with substoichiometric amounts.

The inhibitor-like function also explains why elF5A is not exported by CRM1. CRM1 exports hundreds of proteins by binding to short leucine-rich stretches. In a 
similar binding mode, CRM1 would not shield the hypusine and protect it from undesired interactions.

The structure of the elF5A export complex also illuminates how Xpo4 can mediate export of both isoforms of elF5A. Human elF5A2 is $84 \%$ identical to the major isoform of elF5A. Most of the variations are at the C-terminal domain of elF5A, where Xpo4 does not interact extensively (Figure 4-3). Moreover, the residues interacting with Xpo4 is almost identical in both isoforms. Therefore, Xpo4 does not distinguish between the isoforms and can export both.

A
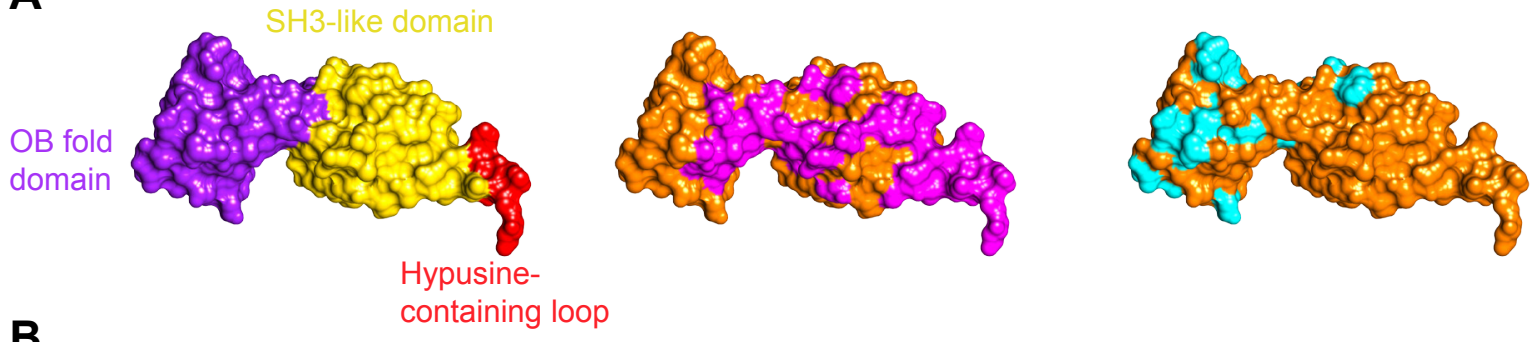

B

10

20

30

40

50

elF5A1 MADDLDFETGDAGA SATFPMQC SALRKNG FVVLKGRPCKIVEMSTSKTG KHGHAKVHLVG EIF5A2 MADE IDFTTGDAGASSTYPMQC SALR KNG F VVLKGRPCKI VEMSTSKTGKHGHAKVHL VG

$\begin{array}{llll}70 & 80 & 90 & 100\end{array}$

elF5A1 I D I F TGKKYED I C P S THNMDVPN I KRNDFQL I G I QDGYLSLLQDSGEVREDLRLPEGDLG eIF5A2 IDIFTGKKYEDICPSTHNMDVPN I KRNDYQL I C I QDG LLLLTETGEVREDLKLPEGELG $130 \quad 140$

elF5A1 KE I EQKYDCGEE I L I TVLSAMTEEAA VA I KAMA K

EIF5A2 KEIEGKYNAGEDVQVS VMCAMSEEYA VA I KPCK-

Figure 4-3 Recognition of elF5A isoforms by Xpo4

(A) elF5As are rendered as surface representation and shown after $180^{\circ}$ rotation around the horizontal axis relative to the view in Figure 3-27 Left: The structural domains of elF5A colored and indicated accordingly. Middle: elF5A is shown in orange and the residues that contact Xpo4 are colored in magenta. Right: The residues that differ in elF5A2 is colored in cyan. (B) Sequence alignment of human elF5A isoforms. Identical residues are highlighted in orange boxes while the different residues in cyan. Magenta circles above the sequence mark the non-conserved residues that contact Xpo4.

\subsubsection{Implications for elF5A function}

As described in section 2.4.2, due to technical reasons, elF5A had been believed to localize to the nucleus. Therefore, a nuclear function for elF5A was an attractive possibility. elF5A was thought to be a shuttling protein, acting as an adaptor molecule for nucleocytoplasmic transport of macromolecules, including the HIV Rev protein (Ruhl et al., 1993; Bevec et al., 1996). Subsequent studies revealed that elF5A did not bind Rev and did not promote export of this protein (Henderson and Percipalle, 1997; Lipowsky et al., 2000). However, due to the ability to bind RNAs in a sequence specific manner, elF5As role as an export adapter for certain 
RNAs have remained a possible function. C-terminal RNA-binding motif of elF5A does not lie at the Xpo4 interaction interface and available for RNA binding. However, given that the RNA interaction of elF5A depends on hypusine (Xu and Chen, 2001) and the Xpo4 dependent export is the only characterized direct export pathway for elF5A (Lipowsky et al., 2000), elF5A's function as an RNA exporter is not rational any longer.

Nevertheless, the elF5A export complex structure does not rule out a nuclear role for elF5A. Acetylation of elF5A at Lys47 has been reported to affect the localization of elF5A (Ishfaq et al., 2012). In the export complex, Lys47 side chain does not contact Xpo4 and the acetylation of this residue seems to be sterically possible. However, the side chain nitrogen makes hydrogen bonding with the main chain oxygen of the hypusine. Since the interaction of this region with Xpo4 is critical, any conformational change upon acetylation would impair export complex formation and cause nuclear accumulation of elF5A. Similarly, any posttranslational modification of elF5A that prevents Xpo4 binding might give rise to nuclear accumulation of elF5A. Nevertheless, any nuclear function would require an active import, which so far has not been characterized for elF5A. elF5A modifications that interrupt Xpo4 binding would be suitable tools for finding an import factor.

\subsubsection{How does Xpo4 recognize other cargoes?}

In the eIF5A export complex, the hypusine plays an important role for Xpo4 binding. Since elF5A is the only protein with this residue, other export cargoes must be recognized differently. Xpo4 has five characteristic insertions. While the acidic loop plays an important role in elF5A binding, roles of the others are elusive. We have already found that the protease sensitive loops are not well conserved among Xpo4 homologs and they are not present in some organisms. Therefore, these regions cannot be part of the conserved export cargo recognition motif. In contrast, insertions at the middle of Xpo4 might be potential regulatory regions important for export cargo recognition. Among the crystallized exportins, CAS and Xpo5 have such long insertions that are important for cargo binding (Matsuura and Stewart, 2004; Okada et al., 2009). 
Sox-type transcription receptors are the only characterized import cargoes of Xpo4. Gontan et al (2009) showed that the basic DNA binding domain of Sox2 and SRY is essential part of the Xpo4 dependent import signal. Given that the inner surface of Xpo4 is acidic (Figure 3-28c), it is tempting to speculate that the import cargoes are recognized through the inner surface of Xpo4. In order to function as a unidirectional importin, RanGTP binding should abolish cargo binding, either directly competing for the binding site or causing conformational changes in the receptor. In canonical importins, the acidic loop has an essential role in cargo binding and release (Conti et al., 2006). In importin $\beta$, Ran competes with the import cargo for binding to the acidic loop. In contrast, in transportin, Ran binding results in an allosteric change of the acidic loop that displaces the cargo. We have already identified HEAT11B of Xpo4 as a potential region for Sox2 recognition motif. This region is very close to the acidic loop (Figure 3-22). Accordingly, it would not be surprising if the acidic loop were part of the import cargo assembly or disassembly mechanism.

Considering the potential importance of the acidic loop for cargo recognition, one can envisage how Xpo4 might act as a bidirectional transporter. All cargoes might bind the acidic loop. The binding can be mutually exclusive or cooperative with RanGTP binding. This would eventually define whether the cargo is an import or export substrate.

\subsubsection{Experimental implications}

elF5A have been the only cargo identified directly by Xpo4 affinity chromatography from a cytoplasmic cell extract. Its high abundance in proliferating cells and its high affinity for Xpo4•RanGTP complex might suggest that elF5A masks the binding (or detection) of other proteins. One approach to identify low abundant or less affine cargoes could be using an elF5A-depleted cell extract. However, this may not be ideal since elF5A depletion would also deplete elF5A interacting proteins. Alternatively, Xpo4 mutants could be utilized for the affinity chromatography. Assuming that the recognition mechanism of other potential export cargoes is different than that of elF5A, it is high likely that one or more of the critical residues of Xpo4 interacts only with elF5A. Then, using Xpo4 mutants that do not bind elF5A would be an elegant way to identify (if there is any) new 
cargoes. We have already characterized several mutants that specifically block elF5A binding (Figure 3-33). Relatively mild mutants (Xpo4 ${ }^{\mathrm{D} 470 \mathrm{~N}}, \mathrm{Xpo} 4^{\mathrm{E} 537 \mathrm{Q}}$ and $X p 04^{\mathrm{S} 631 \mathrm{~A}}$ ) could be the first choice to start with. This hypothesis can be tested with binding assays using the other characterized Xpo4 export cargo, Smad3. In addition, crystallization of the Smad3 export complex would be also informative to see if there is any critical residue that can be exploited for the above-mentioned purpose.

\section{In vitro hypusine-containing elF5A production}

Hypusine is essential for cell viability and elF5A function in translation. Since the identification of elF5A as the hypusine-containing protein, the posttranslational modification of this protein has been extensively studied. The details of the enzymatic reactions, namely deoxyhypusination and hydroxylation, are well known and the protocols describing the in vitro modification of elF5A are available (Park et al., 2011; Wolff et al., 2011). Yet, methods to separate elF5A(Hpu) from elF5A(Dhp) have not existed. During my PhD project, I have established a robust method to separate the fully modified elF5A from the other variants.

The method is based on two features of elF5A variants. The first feature is the presence of the additional positive charge in elF5A(Dhp) and elF5A(Hpu). This allows them to bind cation exchangers stronger than elF5A(Lys). Therefore, it is possible to separate the modified elF5As from the non-modified one in a single chromatographic step. The second feature is the reversibility of the modification of elF5A(Dhp) but not elF5A(Hpu). DHS can use elF5A(Dhp) but not elF5A(Hpu) as a butylamine donor. As a result, in the presence of a suitable acceptor, DHS converts elF5A(Dhp) to elF5A(Lys). In a mixture of elF5As with different modifications, the reversal of the deoxyhypusination reaction produces elF5A(Lys) and elF5A(Hpu). By using the same chromatographic method, the products are separated and fully modified elF5A is obtained.

I used the above-described method to produce large amounts of hypusinated elF5A. The same setup can also be used to determine the composition of an elF5A mixture. By comparing the elution profile (from the cation exchange chromatography) of the mixture with that of elF5A(Lys), one can decide if the mixture contains modified elF5A. Later, the mixture can be subjected to the 
reversal of the deoxyhypusination reaction and analyzed on cation exchange chromatography again. The result will allow the examiner to deduce the complete constituents of the mixture.

\subsection{What have we learned about Xpo7?}

Xpo7 has been proposed to be the second broad-spectrum exportin (after the CRM1), yet only three cargoes have been described in detail. We aimed to discover novel export substrates by using cellular extracts as protein source. After optimization of the Xpo7 affinity chromatography, we utilized a SILAC based mass spectrometry method to identify as many interaction partners as possible.

\subsubsection{Xpo7 is a broad-spectrum exportin}

SILAC analysis has revealed more than twenty proteins interacting with Xpo7 in nuclear conditions (in the presence of RanGTP). All the previously identified Xpo7 interaction partners and characterized export cargoes are in this list, indicating the high coverage of the findings. Moreover, subcellular distribution of the identified interaction partners is mostly cytoplasmic. Therefore, it is an attractive possibility that these proteins are also export cargoes.

Having no detectable sequence and structural similarity between these proteins strongly supports the notion that Xpo7 is a broad-spectrum exportin. This raises the question how Xpo7 can recognize such diverse set of cargoes. CRM1 exports the large number of proteins or protein complexes by recognizing a short leucinerich NES. Employing similar recognition mechanism would be a straightforward approach for Xpo7. However, initial analysis by Mingot et al. (2004) showed that the recognition motif was more than just a short peptide and might involve several positively charged patches on the cargo.

Nevertheless, it is likely that the cargoes have common structural elements that allow them to be recognized by Xpo7. While, it may have not been possible to identify such elements with few cargoes at hand, newly identified proteins will certainly increase the identification probability. Detailed analysis of Xpo7-cargo interactions could allow us to build a novel export signal prediction algorithm, which could be used to discover further Xpo7 cargoes. 


\subsubsection{Xpo7 is a potential bidirectional NTR}

We have not only identified potential export substrates but also several potential import cargoes. For most of the newly discovered potential cargoes, there have not been characterized nuclear import pathways. Therefore, Xpo7-dependent nuclear import is a likely explanation for these proteins. For some of the proteins, we have validated that Xpo7 binding is regulated by Ran. Taken together, although the demonstration of the import activity is elusive, the characterization of Xpo7 as a potential importin makes this receptor the forth candidate of the bidirectional NTR family. This feature of Xpo7 was already suggested after the observation that this receptor bound Ran at mediocre affinities (Kutay et al., 2000).

Xpo7 has been recently associated with erythroid nuclear maturation (Hattangadi et al., 2014). While mature mammalian erythrocytes (red blood cells) do not contain nuclei, their progenitors do. During their differentiation, nuclei of the red blood cell progenitors go through major changes, including chromatin condensation and export of the nuclear proteins, which is followed by enucleation (reviewed by Ji et al., 2011). An erythroid specific isoform of Xpo7 (Xpo7B) is highly expressed at the onset of red blood cell maturation (Hattangadi et al., 2014). Blocking of the Xpo7B expression gives rise to nuclear accumulation of proteins, including histones. Given that Xpo7 is a broad-spectrum exportin, Xpo7 can be considered as the factor required for the cytoplasmic transport of these proteins (Hattangadi et al., 2014). Alternatively, due to its new role as importin, it is tempting to speculate that Xpo7 might import the factors necessary for the early steps of differentiation, such as chromatin condensation. Chromatin condensation requires modification of the histones. Having identified two histone modification enzymes (HAT1 and HDAC8) as potential Xpo7 import cargoes supports this hypothesis.

Investigation of Xpo7 also reveals an interesting observation. Among the four exportins (Xpo4, Xpo6, Xpo7, and Imp13) that are not conserved in all eukaryotes, Xpo4 and Imp13 have been already characterized as bidirectional NTRs. Identification of $\mathrm{Xpo7}$ as a potential bidirectional transport receptor raises the question whether all of these NTRs could be a member of this class of $\operatorname{Imp} \beta$ family. Recent work in our lab has showed that Xpo6 also binds Ran at an intermediate strength, which is a necessity to function as a bidirectional NTR. 


\subsubsection{Functional significance of dimeric Xpo7}

Another interesting finding about Xpo7 is being a dimeric protein under the tested conditions. Imp $\beta / / \mathrm{mp} 7$ heterodimer was the first characterized NTR dimer (Jakel et al., 1999). Recently, human CRM1 has been also shown to form homodimer when binding the HIV Rev response element (Booth et al., 2014). Consequently, Xpo7 is the second example of a homodimeric NTR. Certainly, further investigation is necessary to completely elucidate the function of the homodimer. In this section, I will shortly discuss the possible implications.

Imp $\beta$ and Imp7 are typical importins that are able transport cargoes themselves. However, when Imp $\beta$ and Imp7 form a heterodimer, a unique cargo-binding site is generated, where histone $\mathrm{H} 1$ docks (Jakel et al., 1999). Therefore, by forming different interaction interface, these receptors increase the number of cargoes that can be transported. Similar mechanism can also be envisaged for Xpo7. Xpo7 can import one set of proteins when it is monomer and another set of proteins when it is dimer. By regulating the dimer formation, cell can regulate the nuclear import of proteins that depend on Xpo7 dimer.

Which proteins bind dimeric Xpo7? The answer could be the larger ones. NTRs allow rapid translocation of cargoes by shielding the "inert" surfaces of cargoes while passing through the permeability barrier of the nuclear pore complexes (NPCs). However, as the size of the cargo increases, the transport receptor can no longer cover the inert surface efficiently. This causes a slow down in NPC passage and known as the cargo-effect (Ribbeck and Görlich, 2002). As a result, large proteins require more than one receptor for an efficient transport. Forming an Xpo7 dimer would definitely increase the potential inert area that can be covered. When the lists of Xpo7 binders are analyzed, the protein complexes such as the endosomal retromer, the type II geranylgeranyl transferase and type B histone acetyltransferase complex immediately stand out. Moreover, detailed analysis has revealed that some of the Xpo7 binders, i.e. CutC and 14-3-3 protein sigma, function as homooligomers. Consequently, Xpo7 dimer could be a mechanism for the nucleocytoplasmic transport of these proteins.

The dimer formation can also explain some of the discrepancies that we had in the binding assays. Although we have identified HMBS, MGEA5 and SMUG1 as 
interacting with Xpo7 in a RanGTP sensitive manner (Table 3-7 and Figure 3-47), in the isolated context, they bound Xpo7 even in the presence of RanGTP (Figure 3-48). The results give the impression that RanGTP and the cargo bind Xpo7 at the same time. This might imply that additional factors (that are already present in the extract) are required for complete disassembly of Xpo7•RanGTP from the cargo. Alternatively, if there is an Xpo7 dimer, the cargo and Ran can bind to different subunits of the dimer.

\subsection{Future perspectives}

During my PhD work, I have elucidated the recognition of elF5A and RanGTP by Xpo4. How Xpo4 binds to the other export and import cargoes remain to be determined. I have already obtained promising crystallization conditions with Xpo4-Sox2 complex. The crystals resembled the needle-like crystals that were obtained from the initial export complex crystallization trials. There, the removal of the loop at the $\mathrm{N}$-terminus allowed us to obtain high quality crystals. We shall see if similar engineering of Xpo4 affects the crystal quality of the import complex.

Structure of the unliganded Xpo4 would also be of special interest for in-depth understanding of the cargo induced conformational changes. Due to highly flexible nature of Xpo4, its crystallization might be challenging. Therefore, another structure determination method, cryo-EM, could be utilized. Recent developments in cryo-EM technology allowed high-resolution structures comparable to X-ray crystallography. This technique could especially be useful for identifying the conformational flexibility of unliganded Xpo4.

elF5A is involved in cell proliferation (Hanauske-Abel et al., 1994; Kang and Hershey, 1994; Caraglia et al., 2001; Huang et al., 2007). Although the exact mechanism is not known, overexpression of elF5A isoforms has been associated with increased tumorigenesis in several human cancers (Cracchiolo et al., 2004; Clement et al., 2006; Jasiulionis et al., 2007; Zender et al., 2008; Caraglia et al., 2013). This effect is enhanced upon Xpo4 loss and can be suppressed by re-expression of Xpo4 (Zender et al., 2008). This suggests a rather complex interplay between elF5A and Xpo4. We have already revealed how Xpo4 could prevent elF5A activity in the nucleus. A next challenge will be to discover the other effectors of elF5A in the nucleus. 
Deciphering the mechanisms of cargo recognition by Xpo7 that allow it to be a broad-spectrum transport receptor is the most astonishing future perspectives of this study. We have identified several Xpo7-interacting proteins. Although, we have classified these proteins as potential transport substrates, Xpo7 could be merely a regulator for some of these proteins. Therefore, further analysis is essential to characterize the true import or export cargoes.

There are also many open questions regarding the dimer formation of Xpo7. How does RanGTP regulate dimer formation? Which cargoes are recognized by the Xpo7 dimer? Does Xpo7 form heterodimers with other transport receptors? Further assessment of Xpo7 function will clarify these questions.

Having established an efficient experimental protocol to identify novel transport substrates, we can now test different protein pools from various tissues and developmental stages. Red blood cell progenitors would be the first candidate to test. Similarly, the affinity chromatography protocol can be employed to other NTRs to identify new cargoes or novel functions.

In order to understand how a living cell works, one has to unravel the complete functional networks of proteins in the cell. While the identification of its interaction partners might give a clue on the function of a given protein, the structural information would be the ultimate proof of the function at the molecular level. During my PhD project, I have employed these approaches to study the functions of two nuclear transport receptors, namely Xpo4 and Xpo7. The functions of many more proteins could, in principle, be revealed with similar approaches. With respect to nucleocytoplasmic transport mechanism, this project has already revealed important insights in to the function of the cell. 


\section{MATERIAL AND METHODS}

\subsection{Materials}

\subsubsection{Chemicals}

All chemicals were purchased from Calbiochem (San Diego, California, USA), Carl Roth (Karlsruhe, Germany), Life Technologies GmbH (Darmstadt, Germany), Merck (Darmstadt, Germany), MoBiTec (Göttingen, Germany), New England Biolabs (Ipswich, Massachusetts, USA), Pharmacia (Uppsala, Sweden), Pierce (Rockford, Illinois, USA), Promega (Madison, Wisconsin, USA), Qiagen (Hilden, Germany), Roche Diagnostics (Mannheim, Germany), Serva (Heidelberg, Germany) or Sigma-Aldrich (St. Louis, Missouri, USA).

\subsubsection{Instruments}

\begin{tabular}{|ll|}
\hline Instrument & Manufacturer \\
\hline Arium pro UV water systems & Sartorius (Göttingen, Germany) \\
\hline Äkta Purifier/Explorer & Pharmacia (Uppsala, Sweden) \\
\hline Biophotometer-plus & Eppendorf AG (Hamburg, Germany) \\
\hline Synquad (Cartesian) nanodispenser & Digilab Inc (Marlborough, Massachusetts, USA) \\
\hline Climo-Shaker ISF1-X & Kuhner (Basel, Switzerland) \\
\hline DynaPro NanoStar & Wyatt Technology (Dernbach, Germany) \\
\hline Freedom Evo liquid handling robot & Tecan group (Männedorf, Switzerland) \\
\hline GenePulser & Bio-Rad (Hercules, California, USA) \\
\hline iMac 3.1 GHz Intel Core i5 & Apple (Cupertino, California, USA) \\
\hline Labfors3 Bioreactor & Infors (Bottmingen, Switzerland) \\
\hline Leica MZ6 microscope & Leica Microsystems (Mannheim, Germany) \\
\hline MDF 793 -80 ${ }^{\circ}$ C freezer & Sanyo (Osaka, Japan) \\
\hline MiniDAWN Treos & Wyatt Technology (Dernbach, Germany) \\
\hline NanoDrop ND2000C & Peqlab Biotechnologies (Erlangen, Germany) \\
\hline Odyssey Infrared Imaging System & Licor (Lincoln, Nebraska, USA) \\
\hline Perfection V700 photo scanner & Epson (Long Beach, California, USA) \\
\hline Pitman neo pipettes & Gilson (Middleton, Wisconsin, USA) \\
\hline RM Multi-1 programmable rotator & STARLAB (Hamburg, Germany) \\
\hline $\begin{array}{l}\text { Rock Imager automated imaging } \\
\text { system }\end{array}$ & Formulatrix Inc. (Walthom, Massachusetts, USA) \\
\hline SensoQuest lab cycler & SensoQuest (Göttingen, Germany) \\
\hline Shodex RI-101 & Showa Denko (Minato-ku,Japan) \\
\hline
\end{tabular}




\begin{tabular}{|ll|}
\hline Instrument & Manufacturer \\
Sonifier 450 & Branson (Berkshire, UK) \\
\hline TCS SP5 confocal microscope & Leica Microsystems (Mannheim, Germany) \\
\hline Thermomixer comfort & Eppendorf AG (Hamburg, Germany) \\
\hline UV table & Benda Laborgärate (Wiesloch, Germany) \\
\hline 1260 Infinity Quaternary LC system & Agilent Technologies (Waldbronn, Germany) \\
\hline
\end{tabular}

Table 5-1 Laboratory equipment

\begin{tabular}{|lll|}
\hline Centrifuge & Rotor(s)/Type & Manufacturer \\
\hline Discovery M120 & S55A, S45A, AT3 & Thermo Scientific (Waltham, MA, USA) \\
\hline LYNX 6000 & F9, F12 & Thermo Scientific (Waltham, MA, USA) \\
\hline RC6 plus centrifuge & F9, F10 & Thermo Scientific (Waltham, MA, USA) \\
\hline WX Ultra centrifuge & T647.5, T1250.0 & Thermo Scientific (Waltham, MA, USA) \\
\hline Tabletop centrifuge & 5424 & Eppendorf AG (Hamburg, Germany) \\
\hline $\begin{array}{l}\text { Refrigerated tabletop } \\
\text { centrifuge }\end{array}$ & $5417 R, 5430 R$ & Eppendorf AG (Hamburg, Germany) \\
\hline Multifuge 3I-R & 75006445 & Heraeus (Hanau, Germany) \\
\hline
\end{tabular}

Table 5-2 Centrifuges and rotors

\subsubsection{Software and bioinformatics tools}

\begin{tabular}{|ll|}
\hline Software & Manufacturer \\
\hline Bento 4 & FileMaker Inc. (Santa Clara, California, USA) \\
\hline Bookends & Sonny Software (Checy Chase, Marylands, USA) \\
\hline ccp4 suite & Rutherford Appleton Laboratory (Oxford, UK) \\
\hline UCSF Chimera & UCSF (San Fransisco, California, USA) \\
\hline Illustrator CS5 & Adobe Systems (San Josa, California, USA) \\
\hline ImageJ & National Institute of Health (USA) \\
\hline LASAF & Leica (Mannheim, USA) \\
\hline Lasergene 9 suite & DNASTAR (Madison, Wisconsin, USA) \\
\hline Mac OS X Lion 10.7.5 & Apple (Cupertino, California, USA) \\
\hline Microsoft Office & Microsoft Corporation (Redmond, Washington, USA) \\
\hline Papers2 & Mekentosj (Aalsmeer, Netherlands) \\
\hline Phenix Suite & Lawrence Berkeley Laboratory (Berkeley, California, USA) \\
\hline Photoshop CS5 & Adobe Systems (San Jose, California, USA) \\
\hline Prism 5 & GraphPad Software (La Jolla, California, USA) \\
\hline PyMol & Schrödinger (Portland, Oregon, USA) \\
\hline RockMaker & Formulatrix Inc. (Walthom, Massachusetts, USA) \\
\hline Scaffold 4 & Proteome Software (Portland, Oregon, USA) \\
\hline
\end{tabular}

Table 5-3 Software 


\begin{tabular}{|ll|}
\hline Tool & Website \\
\hline Bioinformatics toolkit & http://toolkit.tuebingen.mpg.de \\
\hline BLAST & http://blast.ncbi.nlm.nih.gov/Blast.cgi \\
\hline COBALT & http://www.ncbi.nlm.nih.gov/tools/cobalt/cobalt.cgi \\
\hline ELM & http://elm.eu.org/search/ \\
\hline Fasta converter & http://genome.nci.nih.gov/tools/reformat.html \\
\hline IUPred & http://iupred.enzim.hu \\
\hline NEB tools & https://www.neb.com/tools-and-resources \\
\hline NLStradamus & http://www.moseslab.csb.utoronto.ca/NLStradamus/ \\
\hline NPS@ & https://npsa-prabi.ibcp.fr/ \\
\hline Nucleotide & http://www.ncbi.nlm.nih.gov/nuccore \\
\hline OligoAnalyzer 3.1 & http://eu.idtdna.com/calc/analyzer \\
\hline OligoCalc & http://www.basic.northwestern.edu/biotools/oligocalc.html \\
\hline Protein Data Bank (PDB) & http://www.rcsb.org/pdb/home/home.do \\
\hline PSIPRED & http://bioinf.cs.ucl.ac.uk/psipred/ \\
\hline PSORT II & http://psort.hgc.jp/form2.html \\
\hline TermiNator & http://www.isv.cnrs-gif.fr/terminator3/index.html \\
\hline The ConSurf Server & http://consurf.tau.ac.il \\
\hline UniProt & http://www.uniprot.org \\
\hline
\end{tabular}

Table 5-4 Online tools

\subsubsection{E.coli strains}

NEB Turbo (C2984, New England Biolabs), NEB 5-alpha (C2987, New England Biolabs) and NEB 10-beta (C3019, New England Biolabs) cells were used for cloning. NEB Express I ${ }^{q}$ (C3037, New England Biolabs), Top10F' (C3030, Life Technologies) and BL21 (C2530, New England Biolabs) cells were used for recombinant protein expression.

\subsubsection{Media for E.coli culture}

\section{LB liquid medium}

$10 \mathrm{~g}$ Tryptone

$5 \mathrm{~g}$ Yeast extract

$10 \mathrm{~g} \mathrm{NaCl}$

$\mathrm{ddH}_{2} \mathrm{O}$ to $1 \mathrm{~L}$

\section{LB plates}

$10 \mathrm{~g}$ Tryptone

$5 \mathrm{~g}$ Yeast extract

\section{YT medium}

16 g Tryptone

$10 \mathrm{~g}$ Yeast extract

$5 \mathrm{~g} \mathrm{NaCl}$

$\mathrm{ddH}_{2} \mathrm{O}$ to $1 \mathrm{~L}$

$50 \mathrm{mM} \mathrm{KH}_{2} \mathrm{PO}_{4}$ and $2 \%$

Glycerol was added to the

expression cultures

\section{TB medium}

$12 \mathrm{~g}$ Tryptone

$24 \mathrm{~g}$ Yeast extract

$4 \mathrm{ml}$ Glycerol

$2.13 \mathrm{~g} \mathrm{KH}_{2} \mathrm{PO}_{4}$

$12.54 \mathrm{~g} \mathrm{~K}_{2} \mathrm{HPO}_{4}$

$\mathrm{ddH}_{2} \mathrm{O}$ to $1 \mathrm{~L}$ 
$10 \mathrm{~g} \mathrm{NaCl}$

$15 \mathrm{~g} \mathrm{Agar}$

dd $_{2} \mathrm{O}$ to $1 \mathrm{~L}$

For the selection and cultivation, liquid media and plates were supplemented with appropriate antibiotics in the following concentrations: Ampicillin $(100 \mu \mathrm{g} / \mathrm{mL})$, kanamycin $(50 \mu \mathrm{g} / \mathrm{mL})$ and spectinomycin $(50 \mu \mathrm{g} / \mathrm{mL})$.

\subsubsection{Buffers and solutions}

10x PfuS buffer

$200 \mathrm{mM}$ Tris/ $\mathrm{HCl} \mathrm{pH} 9.0$

$250 \mathrm{mM} \mathrm{KCl}$

$15 \mathrm{mM} \mathrm{MgSO}_{4}$

$100 \mathrm{mM}\left(\mathrm{NH}_{4}\right)_{2} \mathrm{SO}_{4}$

$1 \%$ Tween-20

$1 \mathrm{mg} / \mathrm{mL}$ BSA

Orange G sample buffer

$10 \mathrm{mM}$ Tris/HCl pH 8.0

10 mM EDTA pH 8.0

$50 \%(w / v)$ Glycerin

$25 \%(w / v)$ Orange G 10x Ligation buffer

$500 \mathrm{mM}$ Tris/HCl pH 7.5

$100 \mathrm{mM} \mathrm{MgCl}_{2}$

$100 \mathrm{mM}$ DTT

$10 \mathrm{mM}$ ATP

$250 \mathrm{mg} / \mathrm{mL}$ BSA

\section{1x TAE buffer}

$4.84 \mathrm{~g}$ Tris Base

$1.14 \mathrm{~mL}$ Acetic acid

$2 \mathrm{ml} 0.5 \mathrm{M}$ EDTA pH 8.0

$\mathrm{ddH}_{2} \mathrm{O}$ to $1 \mathrm{~L}$ 10x SDS-PAGE running buffer $150 \mathrm{~g}$ Glycine

$30 \mathrm{~g}$ Tris Base

$10 \mathrm{~g}$ SDS

$\mathrm{ddH}_{2} \mathrm{O}$ to $1 \mathrm{~L}$

\section{SDS sample buffer}

125 mM Tris/HCl pH 6.8

$3 \%$ SDS

$50 \mathrm{mM}$ DTT

1.0 M Sucrose

$0.1 \mathrm{mg} / \mathrm{ml}$ Bromophenol

Blue
Coomassie stock solution

2\% (w/v) Coomassie

Brilliant Blue G250

$50 \%$ Ethanol
Colloidal Coomassie stock solution

$0.08 \%$ (w/v) Coomassie Brilliant Blue G250

1.6\% Ortho-phosphoric acid

$8 \%(\mathrm{w} / \mathrm{v})\left(\mathrm{NH}_{4}\right)_{2} \mathrm{SO}_{4}$

$20 \%$ Methanol

20x E-Mix

$20 \mathrm{mM}$ HEPES/KOH pH 7.5

$200 \mathrm{mM}$ Creatine phosphate

$1 \mathrm{mg} / \mathrm{mL}$ Creatine kinase

$10 \mathrm{mM}$ ATP

$10 \mathrm{mM}$ GTP

$250 \mathrm{mM}$ Sucrose 10x PBS

$137 \mathrm{mM} \mathrm{NaCl}$

$2.7 \mathrm{mM} \mathrm{KCl}$

$8.1 \mathrm{mM} \mathrm{Na}_{2} \mathrm{HPO}_{4}$

$1.76 \mathrm{mM} \mathrm{KH}_{2} \mathrm{PO}_{4}$

10x Blotting buffer

$3 \mathrm{~g}$ Tris Base

$15 \mathrm{~g}$ Glycine

$200 \mathrm{~mL}$ Methanol

$3 \mathrm{~mL} 10 \%$ SDS

$\mathrm{ddH}_{2} \mathrm{O}$ to $1 \mathrm{~L}$ 
10x TBS

$25 \mathrm{~g}$ Tris $\mathrm{HCl}$

$7.2 \mathrm{~g}$ Tris Base

$87.6 \mathrm{~g} \mathrm{NaCl}$

$\mathrm{dd} \mathrm{H}_{2} \mathrm{O}$ to $1 \mathrm{~L}$

10x M9 salts

$170 \mathrm{~g} \mathrm{Na}_{2} \mathrm{HPO}_{4} \cdot 2 \mathrm{H}_{2} \mathrm{O}$

$60 \mathrm{~g} \mathrm{KH}_{2} \mathrm{PO}_{4}$

$5 \mathrm{~g} \mathrm{NaCl}$

$20 \mathrm{~g} \mathrm{NH}_{4} \mathrm{Cl}$

$\mathrm{ddH}_{2} \mathrm{O}$ to $1 \mathrm{~L}$
Transport buffer

$20 \mathrm{mM}$ HEPES/KOH pH 7.5

$110 \mathrm{mM}$ KOAc

$5 \mathrm{mM} \mathrm{Mg}(\mathrm{OAc})_{2}$

$1 \mathrm{mM}$ EGTA

$250 \mathrm{mM}$ Sucrose

M9 1000x trace elements

$3.7 \mathrm{mg}\left(\mathrm{NH}_{4}\right)_{6} \mathrm{Mo}_{7} \mathrm{O}_{24}$

$24.7 \mathrm{mg} \mathrm{H}_{3} \mathrm{BO}_{3}$

$7.1 \mathrm{mg} \mathrm{CoCl} 2$

$2.5 \mathrm{mg} \mathrm{CuSO}_{4}$

$15.8 \mathrm{mg} \mathrm{MnCl} 2$

$2.9 \mathrm{mg} \mathrm{ZnSO}_{4}$

$\mathrm{ddH}_{2} \mathrm{O}$ to $1 \mathrm{~mL}$

Add $150 \mathrm{mM}$ EDTA and

$4.67 \mathrm{mg} / \mathrm{mL} \mathrm{FeCl}_{3}$ just before adding to medium

\section{RS2 buffer}

$50 \mathrm{mM}$ HEPES/KOH pH8.2

$500 \mathrm{mM} \mathrm{NaCl}$

$5 \mathrm{mM} \mathrm{MgCl}_{2}$
$1 \mathrm{M}$ Tris/HCl pH 7.7

$26.13 \mathrm{~g}$ Tris $\mathrm{HCl}$

$123 \mathrm{~g}$ Tris Base

$\mathrm{dd}_{2} \mathrm{O}$ to $1 \mathrm{~L}$

\section{M9 minimal medium}

$1 \times$ M9 salts

$2 \mathrm{mM} \mathrm{MgSO}_{4}$

$0.4 \%$ Glucose

$0.3 \mathrm{mM} \mathrm{CaCl}_{2}$

$1 \mathrm{mg} / \mathrm{L}$ Thiamine

$1 \mathrm{mg} / \mathrm{L}$ Biotin

1x Trace elements
RS1 buffer

$50 \mathrm{mM}$ Tris $/ \mathrm{HCl} \mathrm{pH} 7.7$

$500 \mathrm{mM} \mathrm{NaCl}$

$2 \mathrm{mM} \mathrm{Mg}(\mathrm{OAc})_{2}$
Binding assay buffer

$50 \mathrm{mM}$ Tris/ $\mathrm{HCl} \mathrm{pH} 7.7$

$100 \mathrm{mM} \mathrm{NaCl}$

$2 \mathrm{mM} \mathrm{Mg}(\mathrm{OAc})_{2}$

$2 \mathrm{mM}$ DTT

\subsubsection{Commercial crystallization screens}

$\mathrm{AmSO}_{4}$, Anions, Cations, Classics, Classics II, Cryos, JSCG+, MbClass, MbClass II, MPD, Nucleix, PACT, PEGs, PEGs II, pHClear, Protein Complex screens were purchased from Qiagen (Hilden, Germany). Index screen was purchase from Hampton Research (Aliso Viejo, California, USA). Pentaerythriol-14 screen was purchased from Jena Bioscience (Jena, Germany). Wizard 1+2 and Wizard 3+4 screens were purchase from Rikagu (Bainbridge Islands, Washington, USA). 


\subsection{Methods}

\subsubsection{DNA construct preparation}

\section{Oligo design and synthesis}

Oligos were designed and optimized using "priming" feature of Seqbuilder (DNASTAR Lasergene Suite 9). Mutations and restriction enzyme cleavage sites were introduced during oligo design. Oligos were ordered from Sigma-Aldrich Chemie GmbH (Steinheim, Germany) as desalted oligos. Upon delivery, oligos were resuspended with water to $100 \mu \mathrm{M}$ and stored at $-20^{\circ} \mathrm{C}$.

\section{Polymerase Chain Reaction (PCR)}

PCR was used for amplification of desired DNA fragments from DNA templates (Mullis et al., 1986).

Typical $100 \mu \mathrm{l}$ volume reaction contained $50 \mathrm{ng}$ of template DNA, $10 \mu \mathrm{l}$ of 10x dNTPs (2.5 mM each), $10 \mu \mathrm{l}$ of 10x PfuS buffer, $1 \mu \mathrm{l}$ of the forward and the reverse primer, $2 \mu \mathrm{l}$ of DMSO, $1 \mu \mathrm{l}$ of PfuS triple mix and was completed to $100 \mu \mathrm{l}$ with $\mathrm{dd}_{2} \mathrm{O}$. PCRs were performed in a SensoQuest lab cycler (Göttingen). A typical example for a PCR protocol is the following:

\begin{tabular}{|c|c|c|c|}
\hline Step & Temperature $\left({ }^{\circ} \mathrm{C}\right)$ & Time & Repeat \\
\hline Initial denaturation & 98.5 & $5^{\prime}$ & 1 \\
\hline Denaturation & 98.5 & $30 "$ & \multirow{3}{*}{30} \\
\hline Annealing & $58-64$ & $30 "$ & \\
\hline Extension & 68 & Varying & \\
\hline Final extension & 68 & $10^{\prime}$ & 1 \\
\hline
\end{tabular}

Table 5-5 Steps of PCR

Annealing temperature was chosen according to primer characteristics and optimized when necessary. Extension time depended on the length of the amplified region; $2 \mathrm{~kb} / \mathrm{min}$ polymerase speed was taken into account when calculating the required time. 


\section{Mutagenesis PCR}

Above mention PCR protocol was performed also for the mutagenesis PCR. After the reaction was completed, $2 \mu \mathrm{L}$ Dnl was added to reaction mixture to digest methylated template DNA and incubated for $1 \mathrm{~h}$ at $37^{\circ} \mathrm{C}$.

\section{DNA gel electrophoresis}

DNA fragments were separated as described in (Sambrook and Russell, 2001) on agarose gels containing $0.8 \%$ to $2 \%$ agarose in $1 \times$ TAE buffer. $1 / 10$ volume Orange G loading buffer was added to DNA samples before loading. In order to visualize, $0.05 \mathrm{\mu g} / \mathrm{ml}$ ethidium bromide was added to liquid agarose. After electrophoresis, DNA bands were visualized on a UV Table (Benda Laborgeräte, Wiesloch), bands corresponding to expected molecular weight were excised for subsequent cloning.

\section{DNA extraction from agarose gels}

Zymoclean gel DNA recovery kit (Zymo Research, Freiburg) was used to recover DNA from manually cut agarose gel bands after electrophoresis.

\section{Determination of DNA concentration}

The concentration of DNA solutions was determined via extinction coefficient at 260nm ( $E_{260}$ ), with $E_{260}=1.0$ corresponding to $50 \mu \mathrm{g} / \mathrm{mL}$ double-stranded DNA (Sambrook and Russell, 2001). Measurements were done using ND-2000C spectrophotometer.

\section{DNA cleavage with restriction enzymes}

Restriction enzymes were obtained from New England Biolabs (Ipswich, USA) and used as recommended by manufacturer to digest $4 \mu \mathrm{g}$ vector or $2 \mu \mathrm{g}$ insert DNA in a reaction volume of $50 \mu \mathrm{L}$.

\section{Ligation of DNA fragments}

\section{Sticky end ligation}

Vector and insert DNA were cleaved with appropriate restriction enzymes. To prevent re-ligation, vector was dephosphorylated with Fast Alkaline Phosphatase 
(FastAP, Fermentas) for $1 \mathrm{~h}$ at $37^{\circ} \mathrm{C}$. DNA fragments were separated on agarose gel. After extraction and purification, vector and insert fragments were ligated by $0.5 \mu \mathrm{l}$ T4 DNA ligase (100 $\mathrm{ng} / \mu \mathrm{L}$, expressed in our lab by Steffen Frey) in a volume of $5 \mu \mathrm{L}$ at $37{ }^{\circ} \mathrm{C}$ for $30 \mathrm{~min}$ in $1 \mathrm{x}$ ligase buffer. $20 \mathrm{ng}$ of vector was incubated with 6 fold molar excess of insert DNA. As a re-ligation control, vector was incubated without insert. $0.5 \mu \mathrm{L}$ of ligation reaction was transformed into electro-competent E.coli cells.

\section{Blunt end ligation}

Blunt end ligation was carried out for the ligation of mutagenesis PCR products. $100 \mathrm{ng}$ of purified DNA was phosphorylated by $0.5 \mu \mathrm{l}$ T4 Polynucleotide kinase ( $300 \mathrm{ng} / \mu \mathrm{L}$, expressed in our lab by Steffen Frey) and ligated by $0.5 \mu \mathrm{l}$ of T4 DNA ligase in a volume of $5 \mu \mathrm{l}$ in $1 \mathrm{x}$ ligase buffer in SensoQuest lab cycler with the following program:

\begin{tabular}{|lcc|}
\hline Step & Temperature $\left({ }^{\circ} \mathbf{C}\right)$ & Time \\
\hline Phosphorylation & 37 & 30 \\
\hline Ligation & 16 & $16 \mathrm{~h}$ \\
\hline Enzyme deactivation & 70 & $10^{\prime}$ \\
\hline
\end{tabular}

Table 5-6 Steps of blunt end ligation reaction

As a negative control, same ligation reaction was carried out without the ligase. $1 \mu \mathrm{L}$ of ligation reaction was transformed into electro-competent E.colicells.

\section{Preparation of electro-competent E.coli cells}

Electro-competent cells were prepared by Gabriele Hawlitschek according to the protocol from (Sambrook and Russell, 2001).

\section{Electroporation of E.coli cells}

$40 \mu \mathrm{L}$ electro-competent $E$.coli cells were combined with $1 \mu \mathrm{L}$ ligation reaction or $250 \mu \mathrm{g}$ plasmid DNA in an electroporation cuvette (165-2086; BioRad, Hercules, USA). Electroporation was performed using MicroPulser (BioRad, Hercules, USA) according to manufacturer's instructions. Cells were recovered for $1 \mathrm{~h}$ at $37^{\circ} \mathrm{C}$ in 1 $\mathrm{ml} 2 \mathrm{YT}, 100 \mu \mathrm{L}$ of cells was plated on LB agar containing the appropriate antibiotic(s) and incubated o/n at $37^{\circ} \mathrm{C}$ 


\section{DNA purification from E.coli cultures}

Small scale plasmid DNA preparations (mini-preps) and large scale plasmid DNA preparations (midi-preps) were done using the NucleoSpin Plasmid kit and NucleoBond PC100 (both Macherey Nagel, Düren) according to the manufacturer's instructions.

\section{Sequencing of plasmid DNA}

All constructs were verified by DNA sequencing (SeqLab, Göttingen), results were analyzed by Seqman (DNASTAR Lasergene Suite 9).

\section{Vectors}

\begin{tabular}{|c|c|}
\hline $\begin{array}{l}\text { Construct } \\
\text { ID }\end{array}$ & Construct Name \\
\hline T145 & H21-TEV-DOHH \\
\hline pKG031 & H14-ZZ-bdSUMO-Ran'-180(Q69L) \\
\hline pKG078 & H14-bdSUMO-ZZ-bdNEDD8-Ran"-180(Q69L) \\
\hline pSF965 & BirA \\
\hline pMA018 & H10-ZZ-bdNEDD8-hsRhoGAP \\
\hline pMA023 & H10-ZZ-bdNEDD8-CutC \\
\hline pMA027 & H10-ZZ-bdNEDD8-hsCutC RKRAR14_18TGSAT \\
\hline pMA028 & H10-ZZ-bdNEDD8-hsXpo7 \\
\hline pMA030 & H10-ZZ-bdNEDD8-hsHAT1 \\
\hline pMA031 & H10-ZZ-bdNEDD8-hsMESH1 \\
\hline pMA032 & H10-ZZ-bdNEDD8-hsNAMPT \\
\hline pMA036 & H10-ZZ-bdNEDD8-hsRBBP7 \\
\hline pMA037 & H10-ZZ-bdNEDD8-hsSMUG1 \\
\hline pMA038 & H10-ZZ-bdNEDD8-hsHMBS \\
\hline pMA039 & H10-ZZ-bdNEDD8-hsMGEA5 \\
\hline pMA040 & H10-ZZ-bdNEDD8-hsHDAC8 \\
\hline pMA042 & H10-ZZ-bdNEDD8-hsRABGGTB \\
\hline pMA061 & H14-ZZ-bdSUMO-GS-hselF5A \\
\hline pMA064 & H10-GFP-TEV-hsDHS \\
\hline pMA066 & H14-bdSUMO-agtg-mmXpo4 \\
\hline pMA071 & H14-ZZ-bdSUMO-hselF5A ${ }^{15-154}$ \\
\hline pMA079 & ZZ-bdNEDD8-ggSox2-H6 \\
\hline pMA083 & H14-bdSUMO-mmXpo4 \\
\hline pMA087 & H14-bdSUMO-mmXpo4 delta931-948 \\
\hline pMA107 & H14-bdSUMO-mmXpo4 ${ }^{\text {delta241-260\&931-948 }}$ \\
\hline pMA111 & H14-bdSUMO-mmXpo4 delta241-260 \\
\hline pMA114 & H14-ZZ-bdSUMO-ggSox2 \\
\hline
\end{tabular}




\begin{tabular}{|c|c|}
\hline $\begin{array}{l}\text { Construct } \\
\text { ID }\end{array}$ & Construct Name \\
\hline pMA119 & H14-ZZ-bdSUMO-ggSox2 HMG box domain \\
\hline pMA131 & H14-bdSUMO-atgt-mmXpo4Y110R \\
\hline pMA134 & H14-bdSUMO-atgt-mmXpo4D395R \\
\hline pMA136 & H14-bdSUMO-atgt-mmXpo4D396R \\
\hline pMA137 & H14-bdSUMO-atgt-mmXpo4E401R \\
\hline pMA139 & H14-bdSUMO-atgt-mmXpo4E904R \\
\hline pMA141 & H14-bdSUMO-atgt-mmXpo4E905R \\
\hline pMA143 & H14-bdSUMO-atgt-mmXpo4D395D396R \\
\hline pMA145 & H14-bdSUMO-atgt-mmXpo4E904E905R \\
\hline pMA146 & H14-bdSUMO-atgt-mmXpo4E390Q \\
\hline pMA147 & H14-bdSUMO-atgt-mmXpo4E390R \\
\hline pMA148 & H14-bdSUMO-atgt-mmXpo4E462R \\
\hline pMA150 & H14-bdSUMO-atgt-mmXpo4E465R \\
\hline pMA151 & H14-bdSUMO-atgt-mmXpo4D470N \\
\hline pMA152 & H14-bdSUMO-atgt-mmXpo4D470R \\
\hline pMA153 & H14-bdSUMO-atgt-mmXpo4E537Q \\
\hline pMA154 & H14-bdSUMO-atgt-mmXpo4E537R \\
\hline pMA155 & H14-bdSUMO-atgt-mmXpo4S631A \\
\hline pMA156 & H14-bdSUMO-atgt-mmXpo4S631R \\
\hline pMA157 & H14-bdSUMO-atgt-mmXpo4S695R \\
\hline pMA161 & H14-bdSUMO-atgt-mmXpo4E906R \\
\hline pMA165 & H14-ZZ-bdSUMO-GS-hselF5AE42A \\
\hline pMA177 & H14-bdSUMO-atgt-mmXpo4D395D396E904E905R \\
\hline pMA189 & H14-ZZ-bdSUMO-hselF5AK50A \\
\hline pMA190 & H14-ZZ-bdSUMO-hselF5AK50R \\
\hline pMA191 & H14-ZZ-bdSUMO-hselF5AH51A \\
\hline pMA215 & H14-bdSUMO-atgt-mmXpo $4^{1-460}$ \\
\hline pMA218 & H14-bdSUMO-atgt-mmXpo $4^{\text {delta } 559-600}$ \\
\hline pMA219 & H14-bdSUMO-atgt-mmXpo $4^{\text {delta } 575-600}$ \\
\hline pMA220 & H14-bdSUMO-atgt-mmXpo $4^{\text {delta654-668 }}$ \\
\hline pMA221 & H14-bdSUMO-atgt-mmXpo4 $4^{\text {delta1036-1040 }}$ \\
\hline pMA222 & H14-bdSUMO-atgt-mmXpo $4^{1-625}$ \\
\hline pMA223 & H14-bdSUMO-atgt-mmXpo $4^{1-691}$ \\
\hline pMA224 & H14-bdSUMO-atgt-mmXpo $4^{1-759}$ \\
\hline pMA225 & H14-bdSUMO-atgt-mmXpo $4^{1-967}$ \\
\hline pMA226 & H14-bdSUMO-atgt-mmXpo $4^{1-993}$ \\
\hline pMA227 & H14-bdSUMO-atgt-mmXpo4 $4^{1-1069}$ \\
\hline pMA228 & H14-bdSUMO-atgt-mmXpo4 $4^{1-1120}$ \\
\hline pKS308 & ED-SUMOvera-H12-mmXpo7A \\
\hline pDG2298 & H14-bdSUMO-Avi-hsXpo7 \\
\hline
\end{tabular}


Table 5-7 List of vectors used in this study

$\mathrm{H} 10, \mathrm{H} 14$ and $\mathrm{H} 21,10,14$ and 21 histidine tags; SUMO, Small ubiquitin-like modifier protease; $\mathrm{ZZ}, \mathrm{Z}$ domain of Staphylococcal Protein A; GFP, green fluorescent protein; TEV, tobacco etch virus protease; NEDD8, Neural precursor cell expressed developmentally down-regulated protein $\underline{8}$; bd, Brachypodium distachyon; $\mathrm{mm}$, Mus musculus; all other proteins were from Homo sapiens

pKG031 and pKG078 were kindly provided by K. Gencalp (our lab), T145 was kindly provided by C. Enke (our lab). pSF965 was kindly provided by Dr. Steffen Frey (our lab). pKS308 was kindly provided by Dr. Katharina Seibel (our lab). pDG2298 was kindly provided by Prof. Dirk Görlich.

\subsubsection{Protein expression and purification}

\section{Expression and purification of Xpo4 variants}

All Xpo4 variants were expressed as $\mathrm{N}$-terminal H14-bdSUMO fusion in E.coli Top10 F' cells. The constructs were transformed into Top10 F' cells and plated on LB agar plates with kanamycin for selection. A single colony was inoculated in $100 \mathrm{ml}$ TB medium supplemented with kanamycin, and pre-culture was grown overnight at $30{ }^{\circ} \mathrm{C}$ with $95 \mathrm{rpm}$ shaking. The pre-culture was diluted to $\mathrm{OD}_{600}$ of 0.3 into $500 \mathrm{ml}$ TB supplemented with kanamycin and incubated at $30{ }^{\circ} \mathrm{C}$ until $\mathrm{OD}_{600}$ reached to 1.0. Cultures were transferred to $21^{\circ} \mathrm{C}$ and grown until $\mathrm{OD}_{600}$ reached to 2.0. Protein expression was induced by the addition of $150 \mu \mathrm{M}$ IPTG and expression was performed for $14-16 \mathrm{~h}$ at $21^{\circ} \mathrm{C}$. Before centrifugation, $5 \mathrm{mM}$ EDTA and $1 \mathrm{mM}$ PMSF were added to the culture to inhibit proteases. Cells were harvested by centrifugation (10 min, $4{ }^{\circ} \mathrm{C}, 5000 \mathrm{rpm}$, F9 rotor) and resuspended in RS1 buffer (50 mM Tris/ $\left.\mathrm{HCl} \mathrm{pH} 7.7,500 \mathrm{mM} \mathrm{NaCl}, 2 \mathrm{mM} \mathrm{Mg}(\mathrm{OAc})_{2}\right)$ supplemented with $5 \%$ glycerol to an $\mathrm{OD}_{600}$ of 100 . Resuspended cells were snap frozen in liquid nitrogen and stored at $-80^{\circ} \mathrm{C}$ or processed immediately. The cells were thawed in warm water, supplemented with $5 \mathrm{mM}$ DTT and lysed with Branson Sonifier W-450 (40\% duty cycle, 9 output power, $2 \times 2$ min on ice). Lysate was cleared by centrifugation $\left(1.5 \mathrm{~h}, 4{ }^{\circ} \mathrm{C}, 41000 \mathrm{rpm}, \mathrm{T} 647.5\right.$ rotor). $15 \mathrm{mM}$ imidazole was added to supernatant and the supernatant was incubated with $1 \mathrm{ml}$ $\mathrm{Ni}$ (II) chelate matrix (24\% Ni-EDTA amide coupled, PEG-passivated silica with $500 \AA$ A pores; matrix was prepared by Prof. Dirk Görlich) equilibrated with RS1 buffer supplemented with $15 \mathrm{mM}$ imidazole for $1.5 \mathrm{~h}$ at $4{ }^{\circ} \mathrm{C}$. Beads were settled and the supernatant was removed. Beads were resuspended with RS1 buffer, transferred to a gravity flow column (Sigma-Aldrich $\mathrm{GmbH}$ ) and washed thoroughly with RS1 buffer containing $25 \mathrm{mM}$ imidazole and $5 \mathrm{mM}$ DTT. Elution was carried 
out by protease cleavage based on (Frey and Görlich, 2014). Briefly, beads were incubated with $200 \mathrm{nM}$ ZZ-tagged bdSENP1 protease (expressed in our lab by Steffen Frey) in RS1 buffer containing $25 \mathrm{mM}$ imidazole and $2 \mathrm{mM}$ DTT for $1.5 \mathrm{~h}$ at $4{ }^{\circ} \mathrm{C} .1 \mathrm{ml}$ RS1 buffer was added from top and the eluate was collected in a single fraction. The eluate consisted untagged protein and ZZ-bdSENP1 protease. The protease was removed by incubating the eluate with $20 \mu \mathrm{L}$ anti-Z affibody matrix (200 нM ZpA963 dimer coupled, GSH quenched Sepharose 2B, matrix was prepared by Prof. Dirk Görlich) for $1 \mathrm{~h}$ at $4{ }^{\circ} \mathrm{C}$. Unbound fraction was subjected to a Superdex 200 16/60 gel filtration column (GE Healthcare) equilibrated with 50 $\mathrm{mM}$ Tris/ $\mathrm{HCl} \mathrm{pH} \mathrm{7.7,} 150 \mathrm{mM} \mathrm{NaCl}, 2 \mathrm{mM} \mathrm{MgOAc}$ and $2 \mathrm{mM}$ DTT. Peak fractions were pooled, supplemented with $250 \mathrm{mM}$ sucrose, aliquoted (to prevent repeated freeze-thaw cycles), snap frozen in liquid nitrogen and stored at $-80^{\circ} \mathrm{C}$. This protocol allowed production of $20-25 \mathrm{mg} \mathrm{Xpo} 4$ from $0.5 \mathrm{~L}$ of expression culture.

For the proteins that were used in crystallization, anion exchange chromatography was performed after the size exclusion chromatography to remove minor proteolytic degradation contaminants. Peak fractions of the gel filtration were pooled; protein sample (diluted to $20 \mathrm{mM} \mathrm{NaCl}$ with $20 \mathrm{mM}$ Tris/ $\mathrm{HCl} \mathrm{pH} \mathrm{7.0,}$ $2 \mathrm{mM} \mathrm{DTT}$ ) was injected to a Mono Q HR $5 / 51 \mathrm{ml}$ column (GE Healthcare) equilibrated with $20 \mathrm{mM}$ Tris/ $\mathrm{HCl} \mathrm{pH} \mathrm{7.0,20} \mathrm{mM} \mathrm{NaCl}$ and $2 \mathrm{mM}$ DTT. Bound proteins were eluted with a linear gradient ending at $50 \mathrm{mM}$ Tris/ $\mathrm{HCl} \mathrm{pH} \mathrm{7.0,300}$ $\mathrm{mM} \mathrm{NaCl}, 2 \mathrm{mM}$ DTT. Pure Xpo4 fraction was directly used for complex formation and crystallization trials.

\section{Expression and purification of Selenomethionine-substituted Xpo4}

The Xpo4 constructs were transformed into BL21 cells and plated on LB agar plates with kanamycin for selection. A single colony was inoculated in $1 \mathrm{~mL} L B$ medium supplemented with kanamycin and grown at $37^{\circ} \mathrm{C}$ for $3-4 \mathrm{~h}$. The culture was centrifuged; pellet was washed twice with M9 medium and resuspended in $1 \mathrm{~mL}$ M9 medium. $200 \mathrm{ml}$ M9 medium was inoculated with the resuspended cells and the pre-culture was incubated $\mathrm{o} / \mathrm{n}$ at $37{ }^{\circ} \mathrm{C}$. Expression cultures were prepared by inoculating $3 \times 50 \mathrm{ml}$ of pre-culture in $3 \times 700 \mathrm{ml}$ fresh $\mathrm{M} 9$ medium. Cultures were grown until $\mathrm{OD}_{600}$ of 0.6 at $37^{\circ} \mathrm{C}$ and then transferred to $20^{\circ} \mathrm{C}$. After $\mathrm{OD}_{600}$ reached to $0.8-1.0,100 \mathrm{mg} / \mathrm{L}$ lysine, phenylalanine and threonine 
(Sigma-Aldrich $\mathrm{GmbH}$ ), $50 \mathrm{mg} / \mathrm{L}$ isoleucine, leucine, valine (Sigma-Aldrich $\mathrm{GmbH}$ ) and $50 \mathrm{mg} / \mathrm{L}$ selenomethionine (Acros Organics) were added. Cultures were incubated at $20{ }^{\circ} \mathrm{C}$ for $15-20 \mathrm{~min}$ and protein expression was induced by the addition of $200 \mu \mathrm{M}$ IPTG. Protein expression took place for 12-14 h. The rest of the protocol was performed as described for native Xpo4. Since the selenomethionine is prone to oxidation, $10 \mathrm{mM}$ DTT was added to the harvested cells before lysis and DTT concentration of all buffers was increased to $5 \mathrm{mM}$ during purification.

\section{Expression and purification of RanGTP}

$\operatorname{Ran}^{5-180}(\mathrm{Q} 69 \mathrm{~L})$ was expressed and purified mainly following the protocol given for Xpo4 variants with the modifications described below. Cells were resuspended in RS2 buffer (50 mM HEPES/KOH pH 8.2, $500 \mathrm{mM} \mathrm{NaCl,} 2 \mathrm{mM} \mathrm{MgCl}$ ) supplemented with $5 \%$ glycerol, $100 \mu \mathrm{M}$ DFP. After binding the protein to $\mathrm{Ni}$ (II) chelate matrix, beads were washed with two column volume (CV) of RS2 buffer containing $25 \mathrm{mM}$ imidazole, $5 \mathrm{mM}$ DTT, and $30 \mu \mathrm{M}$ GTP; then with $2 \mathrm{CV}$ of RS2 buffer containing $25 \mathrm{mM}$ imidazole, $5 \mathrm{~mm}$ DTT, and $1 \mathrm{mM}$ ATP. Final washing was carried out using RS2 buffer containing $25 \mathrm{mM}$ imidazole and $5 \mathrm{mM}$ DTT. Elution was carried out either with untagged bdSENP1 protease or with RS2 buffer containing $500 \mathrm{mM}$ imidazole and $5 \mathrm{mM}$ DTT (for complex formation and crystallization trials). bdSENP1 protease was separated from Ran via cation exchange chromatography. The eluate (diluted to $100 \mathrm{mM} \mathrm{NaCl}$ with $50 \mathrm{mM}$ Tris/ $\mathrm{HCl}, 5 \mathrm{mM} \mathrm{MgOAc}, 2 \mathrm{mM}$ DTT) was bound to a HiTrap SP HP $5 \mathrm{ml}$ column (GE Healthcare) equilibrated with $20 \mathrm{mM}$ Tris/ $\mathrm{HCl} \mathrm{pH} 7.5,40 \mathrm{mM} \mathrm{NaCl}, 5 \mathrm{mM}$ MgOAc, and $2 \mathrm{mM}$ DTT. First, protease was eluted with a gradient ending at 300 $\mathrm{mM} \mathrm{NaCl}$, and then Ran was eluted with $50 \mathrm{mM}$ Tris/ $\mathrm{HCl} \mathrm{pH} \mathrm{7.5,600} \mathrm{mM} \mathrm{NaCl,} 5$ $\mathrm{mM} \mathrm{MgOAc}$, and $2 \mathrm{mM}$ DTT.

The nucleotide state of Ran was confirmed by following protocol. $5 \mathrm{~nm}$ of purified Ran was incubated at $95{ }^{\circ} \mathrm{C}$ for 5 min in order to denature the protein and release the nucleotide. Debris was removed by centrifugation (90 sec, $14000 \mathrm{rpm}, \mathrm{F}-45-$ 30-1 rotor; then $10 \mathrm{~min}, 45000 \mathrm{rpm}$, S45A rotor), supernatant (diluted to $50 \mathrm{mM}$ $\mathrm{NaCl}$ with $50 \mathrm{mM} \mathrm{Tris} / \mathrm{HCl} \mathrm{pH} 8.0$ ) was injected to a Mono Q HR $5 / 51 \mathrm{ml}$ column

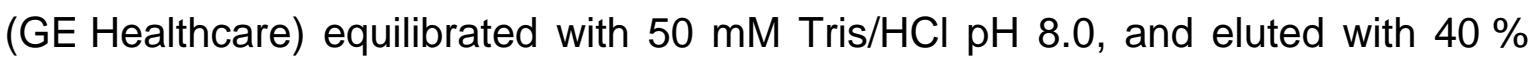


linear gradient with $50 \mathrm{mM}$ Tris/ $\mathrm{HCl} \mathrm{pH}$ 8.0, $1 \mathrm{M} \mathrm{NaCl}$. Elution pattern was compared with that of a $5 \mathrm{nmol}$ GDP-GTP mixture.

\section{Expression and purification of other proteins}

All other proteins were expressed and purified as described for Xpo4 variants with minor modifications. Protein expression was induced at $\mathrm{OD}_{600}$ of $0.8-1.0$. Glycerol was omitted during resuspension. Single immobilized metal affinity chromatography (IMAC) step with $\mathrm{Ni}$ (II) chelate matrix was enough to obtain the desired protein in high concentration and purity. Bound proteins were eluted with RS1 buffer supplemented with $500 \mathrm{mM}$ imidazole and $2 \mathrm{mM}$ DTT.

\section{Expression of proteins with in vivo biotinylation}

Streptavidin-biotin complex is one of the strongest non-covalent complex having a $\mathrm{K}_{\mathrm{d}}$ of $10^{-15} \mathrm{M}$ and this complex is stable at extreme $\mathrm{pH}$, temperature or salt concentrations. We exploited this interaction in the pull down experiments by using a biotinylated protein and a streptavidin-coupled solid phase (streptavidin-agarose, Sigma-Aldrich $\mathrm{GmbH}$ ). For biotinylation, the expression constructs were designed to have an N-terminal Avi-tag (amino acid sequence: GLNDIFEAQKIEWHE), which can be recognized by biotin ligase BirA for covalent attachment of a biotin moiety (Beckett et al., 1999). The expression construct was co-transformed with BirA expression vector (pSF965). Before induction, $20 \mu \mathrm{g} / \mathrm{ml}$ biotin was added to the culture medium. Protein expression was performed as native proteins.

\section{SDS-PAGE}

Recombinant proteins were analyzed by discontinuous sodiumdodecylsulfate polyacrylamide gels (SDS-PAGE) according to the protocols provided by (Sambrook and Russell, 2001). All gels were prepared by Gabriele Hawlitschek and Jürgen Schünemann with the components indicated in Table 5-8. Gels were run for $65 \mathrm{~min}$ at $50 \mathrm{~mA}$ constant current. Afterwards, the proteins were fixed and stained by heating the gel in 3\% acetic acid and 1:100 dilution of Coomassie stock solution. Gels were destained with $\mathrm{H}_{2} \mathrm{O}$ and documented using an EPSON Scanner. 


\begin{tabular}{|l|c|c|c|}
\hline & Heavy Gel (16\%) & Light Gel (7.5\%) & Stacking Gel (4.5\%) \\
\hline Rotiphorese Gel 30 & $108 \mathrm{ml}$ & $51 \mathrm{ml}$ & $15 \mathrm{ml}$ \\
\hline $2 \mathrm{M}$ Tris pH 8.8 & $40 \mathrm{ml}$ & $40 \mathrm{ml}$ & - \\
\hline $0.5 \mathrm{M}$ Tris $\mathrm{pH} 6.8$ & - & - & $15 \mathrm{ml}$ \\
\hline $\mathrm{H}_{2} \mathrm{O}$ & $32 \mathrm{ml}$ & $107 \mathrm{ml}$ & $68 \mathrm{ml}$ \\
\hline $10 \%$ SDS & $2 \mathrm{ml}$ & $2 \mathrm{ml}$ & $1 \mathrm{ml}$ \\
\hline $85 \%$ Glycerol & $8 \mathrm{ml}$ & - & - \\
\hline $2 \mathrm{M}$ Sucrose & $10 \mathrm{ml}$ & - & - \\
\hline TEMED & $130 \mu \mathrm{l}$ & $130 \mu \mathrm{l}$ & $150 \mu \mathrm{l}$ \\
\hline $10 \%$ APS & $2 \times 640 \mu \mathrm{l}$ & $2 \times 640 \mu \mathrm{l}$ & $1.5 \mathrm{ml}$ \\
\hline
\end{tabular}

Table 5-8 Composition of gradient gel solutions

\subsubsection{In vitro modification of elF5A}

\section{Deoxyhypusination}

Protocol for the deoxyhypusination reaction was adapted from (Lipowsky et al., 2000). $20 \mu \mathrm{M}$ untagged recombinant elF5A was incubated with $2 \mu \mathrm{M}$ deoxyhypusine synthase, $2 \mathrm{mM}$ NAD, $2 \mathrm{mM}$ spermidine, $2 \mathrm{mM}$ DTT, $200 \mathrm{mM}$ glycine $\mathrm{pH} 9.0$ for $4 \mathrm{~h}$ at $37^{\circ} \mathrm{C}$. In order to separate deoxyhypusinated elF5A (elF5A(Dhp)) from non-modified elF5A (elF5A(Lys)), buffer was exchanged to

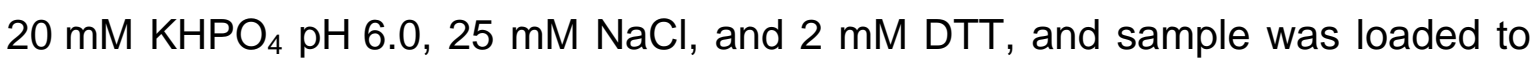
HiTrap SP HP $1 \mathrm{ml}$ column equilibrated with $20 \mathrm{mM} \mathrm{KHPO}_{4} \mathrm{pH}$ 6.0, $25 \mathrm{mM} \mathrm{NaCl}$, $2 \mathrm{mM}$ DTT. Bound proteins were eluted with 15 column volume $40 \%$ linear gradient of $50 \mathrm{mM} \mathrm{KHPO}_{4} \mathrm{pH} 6.0,2 \mathrm{M} \mathrm{NaCl}, 2 \mathrm{mM}$ DTT. The efficiency of modification was $90-95 \%$

When elF5A $\mathrm{A}^{15-154}$ was used for modification, $\mathrm{pH}$ of $\mathrm{KHPO}_{4}$ buffers was adjusted to 6.5

\section{Hydroxylation of elF5A(Dhp)}

Hydroxylation assay was adapted from a published protocol (Park et al., 2011) as follows: $20 \mu \mathrm{M}$ untagged elF5A(Dhp) was incubated with $20 \mu \mathrm{M}$ H14-tagged deoxyhypusine hydroxylase, $2 \mathrm{mM} \mathrm{DTT}, 25 \mathrm{mM}$ Tris/ $\mathrm{HCl} \mathrm{pH} 7.5$ for $4 \mathrm{~h}$ at $37^{\circ} \mathrm{C}$. elF5A was separated from the enzyme by $\mathrm{Ni}$ (II) chelate matrix. Unbound proteins were used in the following reaction. 


\section{Reversal of deoxyhypusination}

For enzymatic removal of deoxyhypusine, $20 \mu \mathrm{M}$ elF5A(Dhp) was incubated with 2 $\mu \mathrm{M}$ deoxyhypusine synthase, $2 \mathrm{mM}$ NAD, 2 mM 1,3-diaminopropane, 2 mM DTT, $200 \mathrm{mM}$ glycine $\mathrm{pH} 9.0$ for $4 \mathrm{~h}$ at $37^{\circ} \mathrm{C}$. Removal of modification was analyzed by cation exchange chromatography as explained for deoxyhypusination. The efficiency of removal was $100 \%$.

End product of the hydroxylation reaction was also subjected to reversal of deoxyhypusination reaction and cation exchange chromatography to determine the efficiency of the hydroxylation reaction.

\section{Large scale hypusination}

Deoxyhypusination and hydroxylation reactions were coupled for the large-scale elF5A hypusination. Since the second reaction is irreversible, coupling pulls the reaction in the forward direction, which increases the efficiency of the deoxyhypusination. Briefly, $160 \mu \mathrm{M}$ H14-ZZ-bdSUMO tagged elF5A was incubated with $4 \mu \mathrm{M}$ deoxyhypusine synthase, $20 \mu \mathrm{M}$ deoxyhypusine hydroxylase, $2 \mathrm{mM}$ NAD, $2.5 \mathrm{mM}$ spermidine, $5 \mathrm{mM}$ DTT, $50 \mathrm{mM}$ Tris $\mathrm{pH} 7.5$ for $16 \mathrm{~h}$ at room temperature. Afterwards, $20 \mu \mathrm{M}$ deoxyhypusine hydroxylase was added and further incubated for $4 \mathrm{~h}$ at $37^{\circ} \mathrm{C}$. elF5A was separated from the enzymes by anti-Z affibody dimer matrix. Bound proteins were eluted with $150 \mathrm{nM}$ bdSUMO protease in $15 \mathrm{mM}$ Tris/ $\mathrm{HCl} \mathrm{pH} 7.0,150 \mathrm{mM} \mathrm{NaCl}$, and $2 \mathrm{mM}$ DTT. Elution (diluted to $50 \mathrm{mM} \mathrm{NaCl}$ with $15 \mathrm{mM}$ Tris/HCl pH 7.0) was bound to a HiTrap SP HP $5 \mathrm{ml}$ column (GE Healthcare) equilibrated with $15 \mathrm{mM}$ Tris/HCl pH 7.0, $20 \mathrm{mM} \mathrm{NaCl}$, and $2 \mathrm{mM}$ DTT. After non-modified elF5A was eluted with $50 \mathrm{mM}$ Tris/HCl pH 7.0, $300 \mathrm{mM} \mathrm{NaCl}$, and $2 \mathrm{mM} \mathrm{DTT}$, modified elF5A was eluted with $50 \mathrm{mM} \mathrm{Tris} / \mathrm{HCl} \mathrm{pH}$ $7.0,600 \mathrm{mM} \mathrm{NaCl}$, and $2 \mathrm{mM}$ DTT.

Reversal of deoxyhypusination reaction was carried out as explained in the previous section with small changes; $100 \mu \mathrm{M}$ modified elF5A and $2.5 \mu \mathrm{M} \mathrm{H} 10$ tagged deoxyhypusine synthase were used for the reaction. elF5A was separated from the enzyme by $\mathrm{Ni}$ (II) chelate matrix. Buffer of the unbound proteins were exchanged to $15 \mathrm{mM}$ Tris/ $\mathrm{HCl} \mathrm{pH} \mathrm{7.0,20} \mathrm{mM} \mathrm{NaCl}$, and $2 \mathrm{mM} \mathrm{DTT}$. elF5A(Hpu) was separated from elF5A(Lys) by cation exchange chromatography as explained 
above. Purified protein was supplemented with $250 \mathrm{mM}$ sucrose, aliquoted, snap frozen in liquid nitrogen and stored at $-80^{\circ} \mathrm{C}$.

\subsubsection{Sample preparation for crystallization}

Protein samples of the highest purity were used in the sample preparation for the crystallization trials. Major effort was to use samples as fresh as possible. Therefore, the protein samples were used either directly after purification or just after one round of freeze thaw cycle. For initial crystallization trials, large-scale samples were prepared. After the initial hits, small-scale preparations were preferred. These preps were aliquoted in $5 \mu \mathrm{L}$ fractions to prevent repeated freeze thaw cycles. In the following sections, I noted down the protocols of the most representative preparations. For simplicity, I described all the protocols for the full-length proteins. When complex formation was carried out with engineered proteins (e.g elF5 $A^{15-154}$, Xpo $4 \Delta N$ ), very same protocol was employed by simply substituting the engineered protein with the full-length protein. When selenomethionine substituted proteins were used, DTT concentration of the buffers was increased to $5 \mathrm{mM}$.

\section{Reconstitution of the elF5A export complexes}

For large-scale preparations, $12.8 \mathrm{mg} \mathrm{Xpo4} \mathrm{was} \mathrm{directly} \mathrm{mixed} \mathrm{with} 4.7 \mathrm{mg} \mathrm{H} 14$ ZZ-bdSUMO tagged RanGTP and $1.9 \mathrm{mg}$ elF5A(Hpu). After $1 \mathrm{~h}$ incubation on ice, sample buffer was exchanged to $15 \mathrm{mM}$ Tris/ $\mathrm{HCl} \mathrm{pH} \mathrm{7.7,48} \mathrm{mM} \mathrm{NaCl,} 2 \mathrm{mM}$ MgOAc and $2 \mathrm{mM}$ DTT (binding buffer). The sample was further incubated on ice for $3 \mathrm{~h}$. Then, $4 \mathrm{ml}$ of the pre-equilibrated anti- $Z$ affibody dimer beads were added and the complex was immobilized via tagged RanGTP. After $2 \mathrm{~h}$ rotation in the cold room, the beads were transferred to a gravity flow column and unbound proteins were removed by washing with the binding buffer. Immobilized RanGTP and the bound proteins were recovered by the protease elution by incubating with $150 \mathrm{nM}$ bdSUMO protease containing binding buffer. The eluate was kept on ice $\mathrm{o} / \mathrm{n}$. Next day; the eluate was centrifuged $10 \mathrm{~min}$ at $14000 \mathrm{rpm}$ and subjected to a Superdex 200 16/60 gel filtration column (GE Healthcare) equilibrated with 15 mM Tris/ $\mathrm{HCl} \mathrm{pH} \mathrm{7.7,} 18 \mathrm{mM} \mathrm{NaCl}, 2 \mathrm{mM} \mathrm{MgOAc}$ and $2 \mathrm{mM}$ DTT. Peak fractions were pooled and concentrated to $12 \mathrm{mg} / \mathrm{ml}$. After concentration, sample was directly used for crystallization. 
For small-scale preparations, $1.38 \mathrm{mg}$ Xpo4 was mixed with $228 \mu \mathrm{g}$ untagged RanGTP and $180 \mu \mathrm{g}$ elF5A(Hpu) and buffer was exchanged to $15 \mathrm{mM}$ Tris/HCl $\mathrm{pH}$ 7.7, $18 \mathrm{mM} \mathrm{NaCl}, 2 \mathrm{mM} \mathrm{MgOAc}$ and $2 \mathrm{mM}$ DTT. The sample was kept at $4{ }^{\circ} \mathrm{C}$ $\mathrm{o} / \mathrm{n}$ for complex formation. Export complex was subjected to size exclusion chromatography as large-scale preparations in order to remove free RanGTP and elF5A(Hpu). Peak fractions were pooled, concentrated to $12 \mathrm{mg} / \mathrm{ml}$ and used for manual crystal settings. The rest was divided in $5 \mu \mathrm{L}$ aliquots, snap frozen in liquid nitrogen and stored at $-80^{\circ} \mathrm{C}$.

\section{Reconstitution of the RanGTP.Xpo4 complex}

Nuclear RanGTP.Xpo4 complex was prepared by following the large-scale elF5A export complex formation protocol with two modifications. First, the complex formation was done by mixing $10.8 \mathrm{mg}$ Xpo4 with $3.5 \mathrm{mg} \mathrm{H} 14-Z Z$-bdSUMO tagged RanGTP. Second, after the size exclusion chromatography, the complex was concentrated to $15 \mathrm{mg} / \mathrm{ml}$ and used directly for crystallization.

\section{Xpo4 preparation}

As explained in 5.2.2, after purification, Xpo4 was cleared by size exclusion chromatography and then polished by anion exchange chromatography. Afterwards, buffer of Xpo4 was exchanged either to $15 \mathrm{mM} \mathrm{Tris/HCl} \mathrm{pH} \mathrm{7.7,}$ $20 \mathrm{mM} \mathrm{NaCl}$ and $2 \mathrm{mM}$ DTT or to $15 \mathrm{mM}$ Tris/ $\mathrm{HCl}, 100 \mathrm{mM} \mathrm{NaCl}$ and $2 \mathrm{mM}$ DTT. After concentrating to $15 \mathrm{mg} / \mathrm{ml}$, Xpo4 was used for crystallization.

\section{Reconstitution of the Sox2 import complex}

Sox2 import complex was prepared by following essentially the same protocol for the reconstitution of the RanGTP•Xpo4 complex. ZZ-bdNEDD8-Sox2-H6 was used for the instead of RanGTP.

\subsubsection{In vitro limited proteolysis}

In order to identify the possible flexible regions, proteins or protein complexes were subjected to (analytical scale) in vitro limited proteolysis. $3 \mu \mathrm{g}$ of protein or protein complex was mixed with trypsin, chymotrypsin and GluC (Roche) in 1:20, $1: 100,1: 500,1: 2500(\mathrm{w} / \mathrm{w})$ protease: substrate ratio in $15 \mu \mathrm{L}$ of $15 \mathrm{mM}$ Tris/HCl $\mathrm{pH} 7.7,18 \mathrm{mM} \mathrm{NaCl}, 2 \mathrm{mM} \mathrm{MgOAc}$. As a control one sample without any protease 
was prepared. The samples were incubated $1 \mathrm{~h}$ at $22{ }^{\circ} \mathrm{C}$ in a thermocycler. Afterwards, $15 \mu \mathrm{L}$ SDS loading buffer (supplemented with $5 \mathrm{mM}$ PMSF and $5 \mathrm{mM}$ EDTA) was added and the samples were boiled (in the thermocycler) at $98^{\circ} \mathrm{C}$ for $10 \mathrm{~min} .10 \mu \mathrm{L}$ of the samples was analyzed by SDS-PAGE followed by Coomassie blue staining.

For the analysis of the digested samples in size exclusion chromatography, $180 \mu \mathrm{g}$ of elF5A $^{15-154} \cdot$ Xpo4 $\cdot$ RanGTP complex was incubated with trypsin $(1: 500 \mathrm{w} / \mathrm{w})$ or chymotrypsin $(1: 100 \mathrm{w} / \mathrm{w})$ in $30 \mu \mathrm{L}$ of $15 \mathrm{mM}$ Tris/ $\mathrm{HCl} \mathrm{pH} \mathrm{7.7,18}$ $\mathrm{mM} \mathrm{NaCl}, 2 \mathrm{mM} \mathrm{MgOAc}, 2 \mathrm{mM}$ DTT for $90 \mathrm{~min}$ at room temperature. $25 \mu \mathrm{L}$ of the sample was injected to a Superdex $20010 / 30$ gel filtration column equilibrated with reaction buffer. Eluted proteins were collected in $200 \mu \mathrm{L}$ fractions and precipitated with $25 \mu \mathrm{L}$ of $100 \%$ TCA. Pellet was resuspended in $25 \mu \mathrm{L}$ SDS loading buffer and $10 \mu \mathrm{L}$ was analyzed by SDS-PAGE followed by Coomassie blue staining.

In order to monitor the effect of the deletions, elF5A export complexes with Xpo4, $X p o 4 \Delta N, X p o 4 \Delta N \& C$ and $X p o 4 \Delta C$ were subjected to analytical scale digestions. $3 \mu \mathrm{g}$ of each of the complexes were incubated with trypsin $(1: 1000 \mathrm{w} / \mathrm{w})$ for $1 \mathrm{~h}$ at $22{ }^{\circ} \mathrm{C}$ and analyzed by following the same procedure above.

\subsubsection{Molecular weight determination with static light scattering (SLS)}

Size exclusion chromatography can be used to estimate the molecular weight of globular proteins. Nevertheless, different conformations or nonspecific interactions with column particles can change the running behavior of the sample. In order to determine the absolute mass, therefore the stoichiometry, of the proteins or protein complexes, we used size exclusion chromatography with multi angle light scattering (SEC-MALS). The principle of the technique is that, for a given particle, the amount of scattered light is proportional to the molecular concentration and the molar mass. In the device, a Superdex 200 10/30 GL (GE Healthcare) gel filtration column is coupled to UV (1260 Infinity, Agilent Technologies, USA) and refractive index (RI) (Shodex RI-101, Showa Denko KK) detectors to measure the protein concentration and to a miniDAWN TREOS (Wyatt Technology) static light scattering detector to measure the intensity of the scattered light. For the 
SEC-MALS experiments, $100 \mu \mathrm{L}$ of $2 \mathrm{mg} / \mathrm{ml}$ sample was used. Astra 6 software (Wyatt Technology) was used to calculate the absolute molecular mass.

\subsubsection{Crystallization}

It is extremely difficult to predict the conditions that would trigger proteins or protein complexes to form well-ordered crystals. Therefore, it is necessary to screen broad range of conditions. We used the crystallization facility of MPI-BPC, run by Dr. Vlad Pena, for automated screening of the crystallization conditions. First, large scale crystallization conditions were screened for initial crystallization hits. Later, the hits were optimized by grid screens. Finally, manual drops were assembled to obtain larger crystals. These crystals were harvested and stored for data collection. Vapor diffusion was the choice of method for all the crystallization setups.

\section{Sparse matrix screening}

We used the commercially available crystallization screens for initial hits. Crystallization drops were set up either in 96 well sitting drop MRC plates (Hampton) using Cartesian Microsys (Genomic Solutions) Nano dispenser robot or in 96 well sitting drop Intelli plates (Ari) using Gryphon (Ari) robot. $60 \mathrm{nl}$ of reservoir solution was pipetted into the wells of the plates followed by $60 \mathrm{nl}$ of protein solution (100 $\mu \mathrm{L}+100 \mu \mathrm{L}$ for Gryphon robot). Plates were sealed tightly and stored at $4{ }^{\circ} \mathrm{C}$ or $20{ }^{\circ} \mathrm{C}$ incubators. Plates were imaged at regular intervals by automated imagers (Formulatrix) and crystal growth was monitored via RockMaker software (Formulatrix).

\section{Grid screening}

When crystals were observed in the sparse matrix screens, corresponding conditions were optimized by grid screens to improve the growth, shape and quality of the initial crystals. RockMaker software (Formulatrix) was used to generate the grid screens. The grid screens were designed by varying the $\mathrm{pH}$ of the buffer and the concentration of the precipitant of the initial condition. One such example is shown below. The plates were pipetted and monitored as described for sparse matrix screens. 


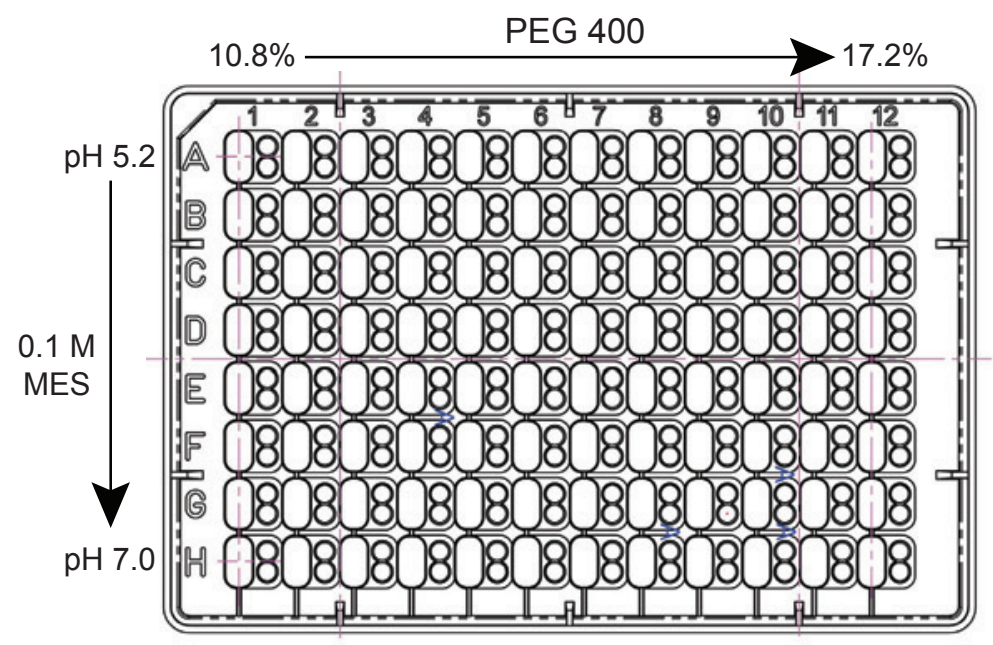

Figure 5-1 An example MRC plate of a grid screen design

Initial crystallization condition is $0.1 \mathrm{M}$ MES pH 6.5, 15\% PEG 400 (PEGll-A2). Original condition was placed to $E 7$ in the grid screen. $\mathrm{pH}$ was varied across columns to cover the whole buffering capacity. On the other hand, precipitant concentration was varied across rows. (MRC plate image was taken from MRC website)

\section{Manual drops}

After the conditions were optimized in the grid screens, further refinement was done with manual drops using 24-well hanging drop Linbro plates. $500 \mu \mathrm{L}$ of the crystallization conditions was pipetted into the well. $1 \mu \mathrm{L}$ of the reservoir solution was placed on a coverslip and $1 \mu \mathrm{L}$ of the protein solution was added. After 2 or 3 such drops were prepared, the coverslip was inverted and greased to the well. The plates were kept at room temperature or placed to $20{ }^{\circ} \mathrm{C}$ incubators. Crystal growth was followed by visual examination under Leica MZ6 microscope (Leica Microsystems).

\section{Seeding}

Seeding technique was used to optimize the crystal growth and the final size of the crystals. Crystallization process can be divided into two, namely, nucleation and crystal growth. Although the aim of the initial screening is to find the optimum condition that supports both, in order to obtain larger crystals, it is necessary to decrease the number of the nucleation events. This can be achieved by modifying the crystallization condition such that it does not allow new nucleation events but supports crystal growth from the present crystals (or seeds). We employed both streakseeding and microseeding for optimization using the manual hanging drop set up explained above. For streakseeding, a cat whisker was passed through the initial crystals to collect small crystals and then a streak line was drawn in the new 
drop to leave the seeds. For microseeding, the initial crystals were taken into 5-10 $\mu \mathrm{L}$ new reservoir solution. Afterwards, crystals were crushed by vortexing, sonication or shaking with a glass bead to create seed stock. The seed stock was diluted in reservoir solution to create dilutions series (ranging from $10^{-1}$ to $10^{-9}$ ) and $1 \mu \mathrm{L}$ of the each dilution was used as precipitant for crystallization.

\section{Crystal mounting}

After crystals reached to a certain size, they had to be harvested in order to store and transport. Crystallization drop containing the desired crystal(s) was slowly exchanged with a cryo protectant solution (i.e 0.1 M MES pH 6.26, 26 \% PEG 400 and $15 \%$ Glycerol). After the crystal was equilibrated with the cryo protectant, the crystal was carefully fished out of the drop using 0.06-0.5 $\mu \mathrm{m}$ sized Nylon loops mounted on a magnetic head (mounted CryoLoops, Hampton Research) and immediately dipped in liquid nitrogen for freezing. Finally, loop was placed into puck and stored in Dewar until further use in the synchrotron.

\section{In situ limited proteolysis}

After the results of the in vitro limited proteolysis experiment, In situ limited proteolysis was performed. Trypsin, chymotrypsin and GluC were diluted with complex buffer to $5.26 \mu \mathrm{g} / \mathrm{ml}, 52.6 \mu \mathrm{g} / \mathrm{ml}$ and $100 \mu \mathrm{g} / \mathrm{ml}$, respectively and each protease was tested separately. $1 \mu \mathrm{L}$ of the protease was mixed with $11 \mu \mathrm{L}$ of the protein solution $(12 \mathrm{mg} / \mathrm{ml})$ on ice just before the crystallization trial. The grid screen crystallization plates (Figure 5-1) were set up as described in sparse matrix screening.

\subsubsection{Structure determination}

All diffraction data were collected at beamline X10SA at the Swiss Light Source (Villigen, Switzerland). All datasets were indexed, integrated and scaled with XDS (Kabsch, 2010). SHELXD was used to locate the 56 selenium sites (out of 66) (Schneider and Sheldrick, 2002). Initial phases were obtained by molecular replacement with PHASER (McCoy, 2007) using Ran (PDB ID 3GJX Monecke et al., 2009) and elF5A (PDB ID 3CPF Tong et al., 2009) as search models. The resulting information and position of selenium atoms were used to obtain the electron density map in AutoSol Wizard (Terwilliger et al., 2009) in Phenix suite 
(Adams et al., 2010). Model building was carried out with resolve and buccaneer (Cowtan, 2006) using AutoBuild Wizard (Terwilliger et al., 2008) in Phenix and with COOT (Emsley and Cowtan, 2004). Iterative cycles of refinement using PHENIX Refine (Afonine et al., 2012) were done after each round of model building and the quality of the model was assessed with MolProbity (Chen et al., 2010). In the final stages, the model was refined against a native data set at a resolution of $3.20 \AA$ to an Rwork of $23.3 \%$ and Rfree of $29.9 \%$. The model has good stereochemistry, with $96.4 \%$ of the residues in the most favored region of the Ramachandran plot and only two residues in the disallowed region.

All figures were prepared using USCF Chimera (http://www.cgl.ucsf.edu/chimera).

\subsubsection{Binding assays}

Binding assays were performed to analyze the protein-protein interactions. Different methods were employed to precipitate the protein complexes. Representative protocols for each different binding assay were written below. Protein concentrations in specific experiments might differ than written here; those cases were clearly indicated in the text and explained in the figure legends.

In all experiments, freshly prepared or aliquoted (single use) proteins were used. Salt contributions of all the components were calculated and $0.5 \mathrm{M} \mathrm{NaCl}$ was used to compensate the salt concentration when necessary. All proteins were mixed in $50 \mathrm{mM}$ Tris/ $\mathrm{HCl} \mathrm{pH} 7.7,2 \mathrm{mM} \mathrm{Mg}(\mathrm{OAc})_{2}$ and $2 \mathrm{mM}$ DTT buffer. Final salt concentration was adjusted to $100 \mathrm{mM}$ after the contributions from the proteins. Therefore, $50 \mathrm{mM}$ Tris/ $\mathrm{HCl} \mathrm{pH} \mathrm{7.7,} 100 \mathrm{mM} \mathrm{NaCl}, 2 \mathrm{mM} \mathrm{Mg}(\mathrm{OAc})_{2}$ and $2 \mathrm{mM}$ DTT was considered as "binding buffer" in the following sections.

When Xpo4-RanGTP interaction was tested, $1 \mu \mathrm{M}$ Xpo4 and $0.75 \mu \mathrm{M} Z Z$ bdNEDD8 tagged RanGTP were used.

When elF5A-Xpo4•RanGTP interaction was tested, $1.25 \mu \mathrm{M}$ elF5A was mixed with $1 \mu \mathrm{M}$ Xpo4 and $0.75 \mu \mathrm{M} Z Z$ Z-bdNEDD8 tagged RanGTP.

In Xpo4 competition experiments, $0.75 \mu \mathrm{M}$ ZZ-NEDD8 tagged RanGTP was incubated with $0.75 \mu \mathrm{M}$ Xpo4 and $0.75 \mu \mathrm{M} \mathrm{H14-Avi-Tev} \mathrm{tagged} \mathrm{Xpo4.}$ 
When HMG domain of Sox2-Xpo4 interaction was tested, $0.75 \mu \mathrm{M}$ Xpo4 and $2 \mu \mathrm{M}$ HMG domain were used.

When elF5A mutants were tested for Xpo4•RanGTP interaction, $0.75 \mu \mathrm{M} \mathrm{H14-ZZ-}$ bdSUMO tagged elF5A mutants were incubated with $1 \mu \mathrm{M}$ Xpo4 and RanGTP, each.

When Xpo7-cargo interactions were tested, $0.5 \mu \mathrm{M}$ of H14-ZZ-bdNEDD8 tagged cargoes was incubated with $0.75 \mathrm{Xpo} 7$ in the presence or absence of $1.5 \mu \mathrm{M}$ RanGTP.

\section{Binding assays with anti-Z affibody dimer beads}

Proteins were mixed in $350 \mu \mathrm{L}$ salt adjusted binding buffer and incubated $2 \mathrm{~h}$ in cold room on SB3 rotator (Bibby Scientific) with $12 \mathrm{rpm}$. Meanwhile $40 \mu \mathrm{L}$ of anti-Z affibody dimer coupled beads were transferred to MoBiCols (MoBiTec) and equilibrated with binding buffer $(2 \times 350 \mu \mathrm{L}) .300 \mu \mathrm{L}$ of the sample was transferred to MoBiCols and incubated with beads to immobilize ZZ-bdNEDD8 or ZZ-bdSUMO tagged cargo. After 90 min rotation, unbound proteins were removed by centrifugation at $700 \mathrm{rpm}$ for $30 \mathrm{sec}$ in a refrigerated tabletop centrifuge. The beads were washed twice with binding buffer $(680+320 \mu \mathrm{L}) .40 \mu \mathrm{L}$ of $250 \mathrm{nM}$ bdNEDD8 or bdSUMO protease supplemented binding buffer was added to each sample and incubated in the cold room for 90 min. Cleaved proteins were collected in a total volume of $100 \mu \mathrm{L}$ as following: After the cleavage $40 \mu \mathrm{L}$ was collected in a $1.5 \mathrm{ml}$ tube by centrifugation, $60 \mu \mathrm{L}$ binding buffer was added to the beads and again centrifuged and collected in the same tube. Then, it was mixed with $100 \mu \mathrm{L}$ SDS loading buffer "Eluate". Remaining proteins (on the beads) were eluted by incubating the beads with $200 \mu \mathrm{L}$ SDS loading buffer for $5 \mathrm{~min}$ at room temperature and centrifugation "Beads". $25 \mu \mathrm{L}$ of the remaining $50 \mu \mathrm{L}$ of the initial sample and $25 \mu \mathrm{L}$ of the unbound protein fraction were mixed with $25 \mu \mathrm{L}$ SDS loading buffer, "Input" and "FT". $10 \mu \mathrm{L}$ of Input, FT, Eluate and Beads were analyzed by SDS-PAGE.

\section{Binding assays with phenyl sepharose}

Proteins were mixed in $350 \mu \mathrm{L}$ salt adjusted binding buffer and incubated $2 \mathrm{~h}$ in the cold room on SB3 rotator (Bibby Scientific) with $12 \mathrm{rpm}$. Meanwhile $40 \mu \mathrm{L}$ of 
phenyl sepharose beads (low substitution, GE Healthcare) were transferred to MoBiCols (MoBiTec) and equilibrated with binding buffer (2x $350 \mu \mathrm{L}) .300 \mu \mathrm{L}$ of the sample was transferred to MoBiCols and incubated with beads to immobilize the NTR. After $2 \mathrm{~h}$ rotation, unbound proteins were removed by centrifugation at $700 \mathrm{rpm}$ for $30 \mathrm{sec}$ in a refrigerated tabletop centrifuge. The beads were washed twice with binding buffer $(680+320 \mu \mathrm{L})$. Bound proteins were eluted by incubating beads with $100 \mu \mathrm{L} 2 \%$ SDS supplemented SDS loading buffer for $5 \mathrm{~min}$ at room temperature. After $100 \mu \mathrm{L}$ is collected in a tube by centrifugation, another $100 \mu \mathrm{L}$ SDS loading buffer was added to the beads and collected in the same tube (total $200 \mu \mathrm{L}$ ) "Eluate". $25 \mu \mathrm{L}$ of the remaining $50 \mu \mathrm{L}$ of the initial sample and $25 \mu \mathrm{L}$ of the unbound protein fraction were mixed with $25 \mu \mathrm{L}$ SDS loading buffer, "Input" and "FT". $10 \mu \mathrm{L}$ of Input, FT and Eluate were analyzed by SDS-PAGE.

\subsubsection{Nuclear export assays}

The assays were performed as previously described (Güttler et al., 2010) with the following modifications: $2 \mu \mathrm{M}$ alexa567-labelled hypusinated elF5A was allowed to diffuse into the nuclei of permeabilized HeLa cells. After $15 \mathrm{~min}$, the mixture was split and $2 \mu \mathrm{M}$ Xpo 4 variant was added. After $30 \mathrm{~min}$, the distribution of elF5A was recorded by confocal fluorescence microscopy.

\subsubsection{Pull down experiments from cytoplasmic Hela extracts}

Cytoplasmic Hela S100 extracts used in this study were kindly provided by Lührmann Lab (Department of Cellular Biochemistry, MPI-BPC). Cytoplasmic SILAC Hela extracts (Heavy and Light) were kindly provided by Dr. Miroslav Nikolov (Mass Spectrometry Research Group, MPI-BPC).

The cytoplasmic extracts from Lührmann Lab were supplemented with $20 \mathrm{mM}$ HEPES/KOH pH 7.5 and $40 \mathrm{mM} \mathrm{NaCl}$. Both extracts were centrifuged at 45000 rpm for $1 \mathrm{~h}$ at $4{ }^{\circ} \mathrm{C}$ using S55A rotor. Supernatant was carefully collected, aliquoted, flash frozen in liquid nitrogen and stored at $-80^{\circ} \mathrm{C}$.

\section{Xpo4 and Xpo7 affinity chromatography}

In vivo biotinylated NTRs were immobilized on streptavidin agarose beads (SigmaAldrich $\mathrm{GmbH}$ ). For each reaction, $20 \mu \mathrm{L}$ of the streptavidin agarose beads were 
used. Beads were taken to a MoBiCol, washed three times with pull down buffer (50 mM Tris/ $\mathrm{HCl} \mathrm{pH} 7.5,50 \mathrm{mM} \mathrm{NaCl}, 5 \mathrm{mM} \mathrm{Mg}(\mathrm{OAc})_{2}, 2 \mathrm{mM}$ DTT) supplemented with $0.005 \%$ digitonin. Buffer was removed by centrifugation for $30 \mathrm{sec}$ at 1000 rpm at $4{ }^{\circ} \mathrm{C}$ in a tabletop centrifuge.

For each reaction $0.5 \mathrm{nmol}$ of biotinylated NTR was immobilized on beads by adding the required amount of protein in $500 \mu \mathrm{L}$ pull down buffer and incubating $1 \mathrm{~h}$ in cold room on SB3 rotator (Bibby Scientific) with $12 \mathrm{rpm}$. After the immobilization, unbound proteins were removed by centrifugation. In order to saturate the available biotin binding sites on the beads, the beads were washed three times with $2 \mu \mathrm{g}$ biotin containing pull down buffer. Finally, beads were washed with pull down buffer $(3 x 500 \mu \mathrm{L})$ and then $20 \mu \mathrm{L}$ of beads were transferred to $1.5 \mathrm{ml}$ tubes.

$1.5 \mathrm{ml}$ of the cytoplasmic Hela extract supplemented with either $5 \mu \mathrm{M}$ RanGTP or same volume of RanGTP buffer was added to the tubes. The samples were incubated in the cold room for $3 \mathrm{~h}$ on SB3 rotator at $8 \mathrm{rpm}$. Afterwards, the tubes were placed on ice for $5 \mathrm{~min}$ and centrifuged for $5 \mathrm{~min}$ at $1000 \mathrm{rpm}$ to settle the beads at the bottom of the tube. Supernatant was carefully removed. Beads were resuspended in $500 \mu \mathrm{L}$ pull down buffer and transferred to MoBiCols. Beads were washed with pull down buffer $(3 \times 500 \mu \mathrm{L})$ allowing them to settle again before the last wash. In order to remove the buffer in the bead volume (dead volume), the samples were centrifuged for $5 \mathrm{sec}$ at $1300 \mathrm{rpm}$. MoBiCols were placed to $1.5 \mathrm{ml}$ tubes and $30 \mu \mathrm{L}$ SDS sample buffer was added to the beads. Tubes were kept at room temperature for $5 \mathrm{~min}$. After centrifugation, another $30 \mu \mathrm{L}$ SDS sample buffer was added and then samples were centrifuged immediately. $10 \mu \mathrm{L}$ was analyzed by SDS-PAGE.

For mass spectrometry-based quantitative proteomics, Xpo7 affinity chromatography was performed with the same procedure above using the cytoplasmic SILAC extracts. For each 'heavy' and 'light' extract, the affinity chromatography was performed in the presence or absence of $5 \mu \mathrm{M}$ RanGTP.

\section{Affinity chromatography with Xpo7 cargoes}

After the identification of potential Xpo7 binders from cytoplasmic Hela extract. We wanted to test if these proteins (RhoGAP, CutC, MESH1, NAMPT, HMBS, 
MGEA5, HDAC8, HAT1, SMUG1 and RabGGTB) can pull down Xpo7 from cytoplasmic Hela extract in a RanGTP dependent or RanGTP sensitive manner. Therefore the affinity chromatography experiment was performed by immobilizing the potential Xpo7 cargoes.

We performed the affinity chromatography experiments with same procedure described in the previous section with some modifications. H14-ZZ-NEDD8 tagged cargoes were used as bait and anti-Z affibody dimer beads were used to capture the baits. $0.5 \mathrm{nmol}$ of the cargo was immobilized and beads were washed. In contrast to NTR chromatography, we did not use $1.5 \mathrm{ml}$ of the extract. Instead, the extract was diluted with same volume of pull down buffer and supplemented with either $3 \mu \mathrm{M}$ RanGTP or same volume of RanGTP buffer. Finally, sample was centrifuged $10 \mathrm{~min}$ at $13000 \mathrm{rpm}$ in a refrigerated tabletop centrifuge. $500 \mu \mathrm{L}$ of the preparation was added to the beads and incubated in the cold room for $3 \mathrm{~h}$ on SB3 rotator at $12 \mathrm{rpm}$. Beads were settled on ice and the centrifuged. Flow through was collected and beads were washed. Cargoes and bound proteins were eluted with NEDD8 protease elution. Simply, beads were incubated with $40 \mu \mathrm{L}$ of $200 \mathrm{nM}$ bdNEDD8 protease for $1 \mathrm{~h}$. Cleaved proteins were recovered in total volume of $80 \mu \mathrm{L}$. $80 \mu \mathrm{L}$ SDS sample buffer was added to the eluate. $10 \mu \mathrm{L}$ of the initial extract was mixed with $80 \mu \mathrm{L}$ SDS sample buffer "Input". $9 \mu \mathrm{L}$ of input and $16 \mu \mathrm{L}$ of the eluates were analyzed by SDS-PAGE.

\subsubsection{Western Blotting}

Whatman papers, SDS gels, nitrocellulose membranes and foam pads were equilibrated in Blotting buffer ( $15 \mathrm{~g} / \mathrm{L}$ Gylcine, $3 \mathrm{~g} / \mathrm{L}$ Tris base in $20 \%$ Methanol and $0.03 \%$ SDS). The blotting cassette was prepared as following: A layer of Whatman paper (3.0 mm, Whatman) was positioned on a foam pad. On top of that the SDS gel was placed, followed by the nitrocellulose membrane (protran, $0.2 \mu \mathrm{m}$ pore size, Schleicher und Schuell $\mathrm{GmbH}$ ). Finally, another layer of Whatman paper was placed and covered with another foam pad. All air bubbles were removed. Blotting cassette was placed into blotting buffer filled chamber such that the SDS gel faced the negative pole of the blotting chamber. It enabled the SDS intercalated proteins to migrate towards the nitrocellulose membrane. Blotting was performed at $4{ }^{\circ} \mathrm{C}$ either for $4 \mathrm{~h}$ at $400 \mathrm{~mA}$ or $16 \mathrm{~h}$ or $100 \mathrm{~mA}$ with gentle stirring. 
Afterwards, the membrane was carefully removed from the cassette and incubated at room temperature first with $1 \mathrm{x}$ TBS for $10 \mathrm{~min}$, then with blocking buffer $(4 \mathrm{~g}$ milk powder in $100 \mathrm{ml} 1 \mathrm{x}$ TBS) for $1 \mathrm{~h}$. Later, the membrane was placed in a primary antibody containing solution (in 1:50000 dilution in blocking buffer) and incubated at least $2 \mathrm{~h}$ (up to $16 \mathrm{~h}$ ) at room temperature. After washing three times with 1x TBS (10 min each), the membrane was incubated with the secondary antibody (Goat $\alpha$-rabbit IRdye, Goat a-mouse IRdye, Licor) containing solution for $1 \mathrm{~h}$ at room temperature. Finally, the membrane was washed with 1x TBS (3x 10 $\mathrm{min}$ ), air-dried and scanned at $800 \mathrm{~nm}$ wavelength using the Odyssey scanner (Licor).

\subsubsection{Mass spectrometry analysis}

Samples to be analyzed by Mass spectrometry were separated on $10 \%$ Bis-Tris gels (NuPAGE, Life Technologies) and stained with Colloidal Coomassie Blue. Individual bands were carefully cut and delivered to Mass spectrometry facility of MPI-BPC for analysis. Final results were analyzed by Scaffold 4.0 software (Proteome Software).

For SILAC analysis, the eluates of the heavy extract with RanGTP and light extract without RanGTP were mixed in equal amounts (Forward experiment). For reverse experiment, the eluates of the light extract with RanGTP and heavy extract without RanGTP were mixed. Forward and reverse experiment samples were separated on $4-12 \%$ gradient SDS-PAGE (NuPAGE, Life Technologies) and stained with Colloidal Coomassie Blue. Each gel lane was cut into 12 equal slices; proteins within the slices were in-gel digested with trypsin and peptides were extracted as described in (Shevchenko et al., 1996). The rest of the analysis was carried out by Samir Karaca (Mass Spectrometry Research Group, MPI-BPC) as previously described in (Wirth et al., 2013). 


\section{REFERENCES}

Adam SA, Marr RS, Gerace L (1990) Nuclear protein import in permeabilized mammalian cells requires soluble cytoplasmic factors. J Cell Biol, 111: 807-816

Adams PD, Afonine PV, Bunkoczi G, Chen VB, Davis IW, Echols N, Headd JJ, Hung LW, Kapral GJ, Grosse-Kunstleve RW, McCoy AJ, Moriarty NW, Oeffner R, Read RJ, Richardson DC, Richardson JS, Terwilliger TC, Zwart PH (2010) PHENIX: a comprehensive Python-based system for macromolecular structure solution. Acta Crystallogr D Biol Crystallogr, 66: 213-221

Afonine PV, Grosse-Kunstleve RW, Echols N, Headd JJ, Moriarty NW, Mustyakimov M, Terwilliger TC, Urzhumtsev A, Zwart PH, Adams PD (2012) Towards automated crystallographic structure refinement with phenix.refine. Acta Crystallogr $D$ Biol Crystallogr, 68: 352-367

Aksu M, Trakhanov S, Görlich D (2016) Structure of the exportin Xpo4 in complex with RanGTP and the hypusine-containing translation factor elF5A. Nat Commun, 7: 11952

Andrade MA, Bork P (1995) HEAT repeats in the Huntington's disease protein. Nat Genet, 11: 115-116

Andrade MA, Petosa C, O'Donoghue SI, Muller CW, Bork P (2001) Comparison of ARM and HEAT protein repeats. $\mathrm{J} \mathrm{Mol} \mathrm{Biol,} \mathrm{309:} \mathrm{1-18}$

Arnold M, Nath A, Wohlwend D, Kehlenbach RH (2006) Transportin is a major nuclear import receptor for c-Fos: a novel mode of cargo interaction. J Biol Chem, 281: 54925499

Arts GJ, Fornerod M, Mattaj IW (1998) Identification of a nuclear export receptor for tRNA. Curr Biol, 8: 305-314

Bayliss R, Littlewood T, Strawn LA, Wente SR, Stewart M (2002) GLFG and FxFG nucleoporins bind to overlapping sites on importin-beta. J Biol Chem, 277: 50597-50606

Becker J, Melchior F, Gerke V, Bischoff FR, Ponstingl H, Wittinghofer A (1995) RNA1 encodes a GTPase-activating protein specific for Gsp1p, the Ran/TC4 homologue of Saccharomyces cerevisiae. J Biol Chem, 270: 11860-11865

Beckett D, Kovaleva E, Schatz PJ (1999) A minimal peptide substrate in biotin holoenzyme synthetase-catalyzed biotinylation. Protein Sci, 8: 921-929

Benne R, Hershey JW (1978) The mechanism of action of protein synthesis initiation factors from rabbit reticulocytes. J Biol Chem, 253: 3078-3087

Bevec D, Jaksche H, Oft M, Wohl T, Himmelspach M, Pacher A, Schebesta M, Koettnitz K, Dobrovnik M, Csonga R, Lottspeich F, Hauber J (1996) Inhibition of HIV-1 replication in lymphocytes by mutants of the Rev cofactor elF-5A. Science, 271: 1858-1860

Bischoff FR, Klebe C, Kretschmer J, Wittinghofer A, Ponstingl H (1994) RanGAP1 induces GTPase activity of nuclear Ras-related Ran. Proc Natl Acad Sci U S A, 91: 2587-2591 
Bischoff FR, Görlich D (1997) RanBP1 is crucial for the release of RanGTP from importin beta-related nuclear transport factors. FEBS Lett, 419: 249-254

Bischoff FR, Ponstingl H (1991a) Mitotic regulator protein RCC1 is complexed with a nuclear ras-related polypeptide. Proc Natl Acad Sci U S A, 88: 10830-10834

Bischoff FR, Ponstingl H (1991b) Catalysis of guanine nucleotide exchange on Ran by the mitotic regulator RCC1. Nature, 354: 80-82

Bohnsack MT, Czaplinski K, Görlich D (2004) Exportin 5 is a RanGTP-dependent dsRNAbinding protein that mediates nuclear export of pre-miRNAs. RNA, 10: 185-191

Bohnsack MT, Regener K, Schwappach B, Saffrich R, Paraskeva E, Hartmann E, Görlich D (2002) Exp5 exports eEF1A via tRNA from nuclei and synergizes with other transport pathways to confine translation to the cytoplasm. EMBO J, 21: 6205-6215

Bonner WM (1975) Protein migration into nuclei. I. Frog oocyte nuclei in vivo accumulate microinjected histones, allow entry to small proteins, and exclude large proteins. $J$ Cell Biol, 64: 421-430

Booth DS, Cheng Y, Frankel AD (2014) The export receptor Crm1 forms a dimer to promote nuclear export of HIV RNA. Elife, 3: e04121

Brownawell AM, Macara IG (2002) Exportin-5, a novel karyopherin, mediates nuclear export of double-stranded RNA binding proteins. J Cell Biol, 156: 53-64

Buggy JJ, Sideris ML, Mak P, Lorimer DD, Mclntosh B, Clark JM (2000) Cloning and characterization of a novel human histone deacetylase, HDAC8. Biochem J, 350 Pt 1: 199-205

Bullock TL, Clarkson WD, Kent HM, Stewart M (1996) The 1.6 angstroms resolution crystal structure of nuclear transport factor 2 (NTF2). J Mol Biol, 260: 422-431

Calado A, Treichel N, Muller EC, Otto A, Kutay U (2002) Exportin-5-mediated nuclear export of eukaryotic elongation factor $1 \mathrm{~A}$ and tRNA. EMBO J, 21: 6216-6224

Cansizoglu AE, Chook YM (2007) Conformational heterogeneity of karyopherin beta2 is segmental. Structure, 15: 1431-1441

Caraglia M, Marra M, Giuberti G, D'Alessandro AM, Budillon A, del Prete S, Lentini A, Beninati S, Abbruzzese A (2001) The role of eukaryotic initiation factor $5 \mathrm{~A}$ in the control of cell proliferation and apoptosis. Amino Acids, 20: 91-104

Caraglia M, Park MH, Wolff EC, Marra M, Abbruzzese A (2013) elF5A isoforms and cancer: two brothers for two functions? Amino Acids, 44: 103-109

Chen KY, Liu AY (1997) Biochemistry and function of hypusine formation on eukaryotic initiation factor 5A. Biol Signals, 6: 105-109

Chen VB, Arendall WB, Headd JJ, Keedy DA, Immormino RM, Kapral GJ, Murray LW, Richardson JS, Richardson DC (2010) MolProbity: all-atom structure validation for macromolecular crystallography. Acta Crystallogr D Biol Crystallogr, 66: 12-21

Chook YM, Blobel G (1999) Structure of the nuclear transport complex karyopherin-beta2Ran x GppNHp. Nature, 399: 230-237 
Chuderland D, Konson A, Seger R (2008) Identification and characterization of a general nuclear translocation signal in signaling proteins. Mol Cell, 31: 850-861

Cingolani G, Petosa C, Weis K, Muller CW (1999) Structure of importin-beta bound to the IBB domain of importin-alpha. Nature, 399: 221-229

Ciufo LF, Brown JD (2000) Nuclear export of yeast signal recognition particle lacking Srp54p by the Xpo1p/Crm1p NES-dependent pathway. Curr Biol, 10: 1256-1264

Clement PM, Johansson HE, Wolff EC, Park MH (2006) Differential expression of elF5A-1 and elF5A-2 in human cancer cells. FEBS J, 273: 1102-1114

Conti E, Muller CW, Stewart M (2006) Karyopherin flexibility in nucleocytoplasmic transport. Curr Opin Struct Biol, 16: 237-244

Cook A, Bono F, Jinek M, Conti E (2007) Structural biology of nucleocytoplasmic transport. Annu Rev Biochem, 76: 647-671

Cook AG, Conti E (2010) Nuclear export complexes in the frame. Curr Opin Struct Biol, 20: $247-252$

Cook AG, Fukuhara N, Jinek M, Conti E (2009) Structures of the tRNA export factor in the nuclear and cytosolic states. Nature, 461: 60-65

Coutavas E, Ren M, Oppenheim JD, D'Eustachio P, Rush MG (1993) Characterization of proteins that interact with the cell-cycle regulatory protein Ran/TC4. Nature, 366: 585-587

Cowtan K (2006) The Buccaneer software for automated model building. 1. Tracing protein chains. Acta Crystallogr D Biol Crystallogr, 62: 1002-1011

Cracchiolo BM, Heller DS, Clement PM, Wolff EC, Park MH, Hanauske-Abel HM (2004) Eukaryotic initiation factor 5A-1 (elF5A-1) as a diagnostic marker for aberrant proliferation in intraepithelial neoplasia of the vulva. Gynecol Oncol, 94: 217-222

Dean KA, von Ahsen O, Görlich D, Fried HM (2001) Signal recognition particle protein 19 is imported into the nucleus by importin 8 (RanBP8) and transportin. J Cell Sci, 114: 3479-3485

Doerfel LK, Wohlgemuth I, Kothe C, Peske F, Urlaub H, Rodnina MV (2013) EF-P is essential for rapid synthesis of proteins containing consecutive proline residues. Science, 339: 85-88

Dong X, Biswas A, Suel KE, Jackson LK, Martinez R, Gu H, Chook YM (2009) Structural basis for leucine-rich nuclear export signal recognition by CRM1. Nature, 458: 1136-1141

Dorfman J, Macara IG (2008) STRADalpha regulates LKB1 localization by blocking access to importin-alpha, and by association with Crm1 and exportin-7. Mol Biol Cell, 19: $1614-1626$

Eibauer M, Pellanda M, Turgay Y, Dubrovsky A, Wild A, Medalia O (2015) Structure and gating of the nuclear pore complex. Nat Commun, 6: 7532

Emsley P, Cowtan K (2004) Coot: model-building tools for molecular graphics. Acta Crystallogr D Biol Crystallogr, 60: 2126-2132 
Farnsworth CC, Seabra MC, Ericsson LH, Gelb MH, Glomset JA (1994) Rab geranylgeranyl transferase catalyzes the geranylgeranylation of adjacent cysteines in the small GTPases Rab1A, Rab3A, and Rab5A. Proc Natl Acad Sci U S A, 91: 11963-11967

Fischer U, Huber J, Boelens WC, Mattaj IW, Luhrmann R (1995) The HIV-1 Rev activation domain is a nuclear export signal that accesses an export pathway used by specific cellular RNAs. Cell, 82: 475-483

Fornerod M, Ohno M, Yoshida M, Mattaj IW (1997) CRM1 is an export receptor for leucine-rich nuclear export signals. Cell, 90: 1051-1060

Frey S, Görlich D (2007) A saturated FG-repeat hydrogel can reproduce the permeability properties of nuclear pore complexes. Cell, 130: 512-523

Frey S, Görlich D (2014) A new set of highly efficient, tag-cleaving proteases for purifying recombinant proteins. J Chromatogr A, 1337: 95-105

Gall JG (1967) Octagonal nuclear pores. J Cell Biol, 32: 391-399

Gao Y, Wells L, Comer FI, Parker GJ, Hart GW (2001) Dynamic O-glycosylation of nuclear and cytosolic proteins: cloning and characterization of a neutral, cytosolic beta- $\mathrm{N}$ acetylglucosaminidase from human brain. J Biol Chem, 276: 9838-9845

Gontan C, Güttler T, Engelen E, Demmers J, Fornerod M, Grosveld FG, Tibboel D, Görlich D, Poot RA, Rottier RJ (2009) Exportin 4 mediates a novel nuclear import pathway for Sox family transcription factors. J Cell Biol, 185: 27-34

Gordon ED, Mora R, Meredith SC, Lee C, Lindquist SL (1987) Eukaryotic initiation factor 4D, the hypusine-containing protein, is conserved among eukaryotes. J Biol Chem, 262: 16585-16589

Görlich D, Dabrowski M, Bischoff FR, Kutay U, Bork P, Hartmann E, Prehn S, Izaurralde E (1997) A novel class of RanGTP binding proteins. J Cell Biol, 138: 65-80

Görlich D, Henklein P, Laskey RA, Hartmann E (1996a) A 41 amino acid motif in importinalpha confers binding to importin-beta and hence transit into the nucleus. EMBO J, 15: $1810-1817$

Görlich D, Kostka S, Kraft R, Dingwall C, Laskey RA, Hartmann E, Prehn S (1995) Two different subunits of importin cooperate to recognize nuclear localization signals and bind them to the nuclear envelope. Curr Biol, 5: 383-392

Görlich D, Kutay U (1999) Transport between the cell nucleus and the cytoplasm. Annu Rev Cell Dev Biol, 15: 607-660

Görlich D, Pante N, Kutay U, Aebi U, Bischoff FR (1996b) Identification of different roles for RanGDP and RanGTP in nuclear protein import. EMBO J, 15: 5584-5594

Görlich D, Prehn S, Laskey RA, Hartmann E (1994) Isolation of a protein that is essential for the first step of nuclear protein import. Cell, 79: 767-778

Gregio AP, Cano VP, Avaca JS, Valentini SR, Zanelli CF (2009) elF5A has a function in the elongation step of translation in yeast. Biochem Biophys Res Commun, 380: 785-790 
Grunwald M, Lazzaretti D, Bono F (2013) Structural basis for the nuclear export activity of Importin13. EMBO J, 32: 899-913

Gutierrez E, Shin BS, Woolstenhulme CJ, Kim JR, Saini P, Buskirk AR, Dever TE (2013) elF5A promotes translation of polyproline motifs. Mol Cell, 51: 35-45

Güttler T, Görlich D (2011) Ran-dependent nuclear export mediators: a structural perspective. EMBO J, 30: 3457-3474

Güttler T, Madl T, Neumann P, Deichsel D, Corsini L, Monecke T, Ficner R, Sattler M, Görlich D (2010) NES consensus redefined by structures of PKI-type and Rev-type nuclear export signals bound to CRM1. Nat Struct Mol Biol, 17: 1367-1376

Hanauske-Abel HM, Park MH, Hanauske AR, Popowicz AM, Lalande M, Folk JE (1994) Inhibition of the G1-S transition of the cell cycle by inhibitors of deoxyhypusine hydroxylation. Biochim Biophys Acta, 1221: 115-124

Hattangadi SM, Martinez-Morilla S, Patterson HC, Shi J, Burke K, Avila-Figueroa A, Venkatesan S, Wang J, Paulsen K, Görlich D, Murata-Hori M, Lodish HF (2014) Histones to the cytosol: exportin 7 is essential for normal terminal erythroid nuclear maturation. Blood, 124: 1931-1940

Haushalter KA, Todd Stukenberg MW, Kirschner MW, Verdine GL (1999) Identification of a new uracil-DNA glycosylase family by expression cloning using synthetic inhibitors. Curr Biol, 9: 174-185

Henderson BR, Percipalle P (1997) Interactions between HIV Rev and nuclear import and export factors: the Rev nuclear localisation signal mediates specific binding to human importin-beta. J Mol Biol, 274: 693-707

Ho JH, Kallstrom G, Johnson AW (2000) Nmd3p is a Crm1p-dependent adapter protein for nuclear export of the large ribosomal subunit. J Cell Biol, 151: 1057-1066

Hopper AK, Traglia HM, Dunst RW (1990) The yeast RNA1 gene product necessary for RNA processing is located in the cytosol and apparently excluded from the nucleus. $J$ Cell Biol, 111: 309-321

Hu E, Chen Z, Fredrickson T, Zhu Y, Kirkpatrick R, Zhang GF, Johanson K, Sung CM, Liu R, Winkler J (2000) Cloning and characterization of a novel human class I histone deacetylase that functions as a transcription repressor. J Biol Chem, 275: 15254-15264

Huang Y, Higginson DS, Hester L, Park MH, Snyder SH (2007) Neuronal growth and survival mediated by elF5A, a polyamine-modified translation initiation factor. Proc Natl Acad Sci U S A, 104: 4194-4199

Huber J, Cronshagen U, Kadokura M, Marshallsay C, Wada T, Sekine M, Luhrmann R (1998) Snurportin1, an m3G-cap-specific nuclear import receptor with a novel domain structure. EMBO J, 17: 4114-4126

Hulsmann BB, Labokha AA, Görlich D (2012) The permeability of reconstituted nuclear pores provides direct evidence for the selective phase model. Cell, 150: 738-751

Hutten S, Kehlenbach RH (2007) CRM1-mediated nuclear export: to the pore and beyond. Trends Cell Biol, 17: 193-201 
Imaoka N, Nakajima T (1973) Hypusine, N6-(4-amino-2-hydroxybutyl)-2,6diaminohexanoic acid, in tissue proteins of mammals. Biochim Biophys Acta, 320: 97-103

Ishfaq M, Maeta K, Maeda S, Natsume T, Ito A, Yoshida M (2012) Acetylation regulates subcellular localization of eukaryotic translation initiation factor $5 \mathrm{~A}$ (elF5A). FEBS Lett, 586: $3236-3241$

Ishizawa J, Kojima K, Hail NJ, Tabe Y, Andreeff M (2015) Expression, function, and targeting of the nuclear exporter chromosome region maintenance 1 (CRM1) protein. Pharmacol Ther, 153: 25-35

Izaurralde E, Kutay U, von Kobbe C, Mattaj IW, Görlich D (1997) The asymmetric distribution of the constituents of the Ran system is essential for transport into and out of the nucleus. EMBO J, 16: 6535-6547

Izaurralde E, Lewis J, Gamberi C, Jarmolowski A, McGuigan C, Mattaj IW (1995) A capbinding protein complex mediating U snRNA export. Nature, 376: 709-712

Jakel S, Albig W, Kutay U, Bischoff FR, Schwamborn K, Doenecke D, Görlich D (1999) The importin beta/importin 7 heterodimer is a functional nuclear import receptor for histone H1. EMBO J, 18: 2411-2423

Jakel S, Görlich D (1998) Importin beta, transportin, RanBP5 and RanBP7 mediate nuclear import of ribosomal proteins in mammalian cells. EMBO J, 17: 4491-4502

Jakel S, Mingot JM, Schwarzmaier P, Hartmann E, Görlich D (2002) Importins fulfil a dual function as nuclear import receptors and cytoplasmic chaperones for exposed basic domains. EMBO J, 21: 377-386

Jao DL, Chen KY (2006) Tandem affinity purification revealed the hypusine-dependent binding of eukaryotic initiation factor $5 \mathrm{~A}$ to the translating $80 \mathrm{~S}$ ribosomal complex. $J$ Cell Biochem, 97: 583-598

Jao DL, Yu Chen K (2002) Subcellular localization of the hypusine-containing eukaryotic initiation factor $5 \mathrm{~A}$ by immunofluorescent staining and green fluorescent protein tagging. $J$ Cell Biochem, 86: 590-600

Jasiulionis MG, Luchessi AD, Moreira AG, Souza PP, Suenaga AP, Correa M, Costa CA, Curi R, Costa-Neto CM (2007) Inhibition of eukaryotic translation initiation factor $5 \mathrm{~A}$ (elF5A) hypusination impairs melanoma growth. Cell Biochem Funct, 25: 109-114

Jenkins ZA, Haag PG, Johansson HE (2001) Human elF5A2 on chromosome 3q25-q27 is a phylogenetically conserved vertebrate variant of eukaryotic translation initiation factor 5A with tissue-specific expression. Genomics, 71: 101-109

Ji P, Murata-Hori M, Lodish HF (2011) Formation of mammalian erythrocytes: chromatin condensation and enucleation. Trends Cell Biol, 21: 409-415

Sambrook J, Russell DW. (2001) Molecular Cloning: A Laboratory Manual. Cold Spring Harbor Laboratory Press, Cold Spring Harbor, NY, USA.

Kabsch W (2010) XDS. Acta Crystallogr D Biol Crystallogr, 66: 125-132

Kalderon D, Roberts BL, Richardson WD, Smith AE (1984) A short amino acid sequence able to specify nuclear location. Cell, 39: 499-509 
Kang HA, Hershey JW (1994) Effect of initiation factor elF-5A depletion on protein synthesis and proliferation of Saccharomyces cerevisiae. J Biol Chem, 269: 3934-3940

Kataoka N, Bachorik JL, Dreyfuss G (1999) Transportin-SR, a nuclear import receptor for SR proteins. J Cell Biol, 145: 1145-1152

Kemper WM, Berry KW, Merrick WC (1976) Purification and properties of rabbit reticulocyte protein synthesis initiation factors M2Balpha and M2Bbeta. J Biol Chem, 251: $5551-5557$

Kim KK, Hung LW, Yokota H, Kim R, Kim SH (1998) Crystal structures of eukaryotic translation initiation factor $5 \mathrm{~A}$ from Methanococcus jannaschii at $1.8 \mathrm{~A}$ resolution. Proc Natl Acad Sci U S A, 95: 10419-10424

Kim MS, Pinto SM, Getnet D, Nirujogi RS, Manda SS, Chaerkady R, Madugundu AK, Kelkar DS, Isserlin R, Jain S, Thomas JK, Muthusamy B, Leal-Rojas P, Kumar P, Sahasrabuddhe NA, Balakrishnan L, Advani J, George B, Renuse S, Selvan LD, Patil AH, Nanjappa V, Radhakrishnan A, Prasad S, Subbannayya T, Raju R, Kumar M, Sreenivasamurthy SK, Marimuthu A, Sathe GJ, Chavan S, Datta KK, Subbannayya Y, Sahu A, Yelamanchi SD, Jayaram S, Rajagopalan P, Sharma J, Murthy KR, Syed N, Goel R, Khan AA, Ahmad S, Dey G, Mudgal K, Chatterjee A, Huang TC, Zhong J, Wu X, Shaw PG, Freed D, Zahari MS, Mukherjee KK, Shankar S, Mahadevan A, Lam H, Mitchell CJ, Shankar SK, Satishchandra P, Schroeder JT, Sirdeshmukh R, Maitra A, Leach SD, Drake CG, Halushka MK, Prasad TS, Hruban RH, Kerr CL, Bader GD, lacobuzio-Donahue CA, Gowda H, Pandey A (2014) A draft map of the human proteome. Nature, 509: 575-581

Klebe C, Bischoff FR, Ponstingl H, Wittinghofer A (1995) Interaction of the Nuclear GTPBinding Protein Ran with Its Regulatory Proteins RCC1 and RanGAP1 - Biochemistry (ACS Publications). Biochemistry,

Kose S, Imamoto N, Tachibana T, Shimamoto T, Yoneda Y (1997) Ran-unassisted nuclear migration of a $97-\mathrm{kD}$ component of nuclear pore-targeting complex. J Cell Biol, 139: $841-849$

Koyama M, Matsuura Y (2010) An allosteric mechanism to displace nuclear export cargo from CRM1 and RanGTP by RanBP1. EMBO J, 29: 2002-2013

Kurisaki A, Kurisaki K, Kowanetz M, Sugino H, Yoneda Y, Heldin C-H, Moustakas A (2006) The mechanism of nuclear export of Smad3 involves exportin 4 and Ran. Mol Cell Biol, 26: 1318-1332

Kutay U, Bischoff FR, Kostka S, Kraft R, Görlich D (1997) Export of importin alpha from the nucleus is mediated by a specific nuclear transport factor. Cell, 90: 1061-1071

Kutay U, Hartmann E, Treichel N, Calado A, Carmo-Fonseca M, Prehn S, Kraft R, Görlich D, Bischoff FR (2000) Identification of two novel RanGTP-binding proteins belonging to the importin beta superfamily. J Biol Chem, 275: 40163-40168

Kutay U, Lipowsky G, Izaurralde E, Bischoff FR, Schwarzmaier P, Hartmann E, Görlich D (1998) Identification of a tRNA-specific nuclear export receptor. Mol Cell, 1: 359-369

Kyrpides NC, Woese CR (1998) Universally conserved translation initiation factors. Proc Natl Acad Sci U S A, 95: 224-228 
Lam MH, Briggs LJ, Hu W, Martin TJ, Gillespie MT, Jans DA (1999) Importin beta recognizes parathyroid hormone-related protein with high affinity and mediates its nuclear import in the absence of importin alpha. J Biol Chem, 274: 7391-7398

Lassak J, Keilhauer EC, Furst M, Wuichet K, Godeke J, Starosta AL, Chen JM, SogaardAndersen L, Rohr J, Wilson DN, Haussler S, Mann M, Jung K (2015) Argininerhamnosylation as new strategy to activate translation elongation factor P. Nat Chem Biol, 11: $266-270$

Lee SB, Park JH, Kaevel J, Sramkova M, Weigert R, Park MH (2009) The effect of hypusine modification on the intracellular localization of elF5A. Biochem Biophys Res Commun, 383: 497-502

Lee SJ, Matsuura Y, Liu SM, Stewart M (2005) Structural basis for nuclear import complex dissociation by RanGTP. Nature, 435: 693-696

Lee YB, Park MH, Folk JE (1995) Diamine and triamine analogs and derivatives as inhibitors of deoxyhypusine synthase: synthesis and biological activity. J Med Chem, 38: 3053-3061

Li J, Ji C, Chen J, Yang Z, Wang Y, Fei X, Zheng M, Gu X, Wen G, Xie Y, Mao Y (2005) Identification and characterization of a novel Cut family cDNA that encodes human copper transporter protein CutC. Biochem Biophys Res Commun, 337: 179-183

Lipowsky G, Bischoff FR, Izaurralde E, Kutay U, Schafer S, Gross HJ, Beier H, Görlich D (1999) Coordination of tRNA nuclear export with processing of tRNA. RNA, 5: 539-549

Lipowsky G, Bischoff FR, Schwarzmaier P, Kraft R, Kostka S, Hartmann E, Kutay U, Görlich D (2000) Exportin 4: a mediator of a novel nuclear export pathway in higher eukaryotes. EMBO J, 19: 4362-4371

Liu J, Perumal NB, Oldfield CJ, Su EW, Uversky VN, Dunker AK (2006) Intrinsic disorder in transcription factors. Biochemistry, 45: 6873-6888

Lund E, Guttinger S, Calado A, Dahlberg JE, Kutay U (2004) Nuclear export of microRNA precursors. Science, 303: 95-98

Magdolen V, Klier H, Wohl T, Klink F, Hirt H, Hauber J, Lottspeich F (1994) The function of the hypusine-containing proteins of yeast and other eukaryotes is well conserved. $\mathrm{Mol}$ Gen Genet, 244: 646-652

Mahajan R, Delphin C, Guan T, Gerace L, Melchior F (1997) A small ubiquitin-related polypeptide involved in targeting RanGAP1 to nuclear pore complex protein RanBP2. Cell, 88: $97-107$

Makowski AM, Dutnall RN, Annunziato AT (2001) Effects of acetylation of histone H4 at lysines 8 and 16 on activity of the Hat1 histone acetyltransferase. J Biol Chem, 276: 43499-43502

Masaoka A, Matsubara M, Hasegawa R, Tanaka T, Kurisu S, Terato H, Ohyama $\mathrm{Y}$, Karino N, Matsuda A, Ide H (2003) Mammalian 5-formyluracil-DNA glycosylase. 2. Role of SMUG1 uracil-DNA glycosylase in repair of 5-formyluracil and other oxidized and deaminated base lesions. Biochemistry, 42: 5003-5012 
Matsuura Y, Stewart M (2004) Structural basis for the assembly of a nuclear export complex. Nature, 432: 872-877

Matunis MJ, Coutavas E, Blobel G (1996) A novel ubiquitin-like modification modulates the partitioning of the Ran-GTPase-activating protein RanGAP1 between the cytosol and the nuclear pore complex. J Cell Biol, 135: 1457-1470

McCoy AJ (2007) Solving structures of protein complexes by molecular replacement with Phaser. Acta Crystallogr D Biol Crystallogr, 63: 32-41

Melchior F, Paschal B, Evans J, Gerace L (1993) Inhibition of nuclear protein import by nonhydrolyzable analogues of GTP and identification of the small GTPase Ran/TC4 as an essential transport factor. J Cell Biol, 123: 1649-1659

Mingot JM, Bohnsack MT, Jakle U, Görlich D (2004) Exportin 7 defines a novel general nuclear export pathway. EMBO J, 23: 3227-3236

Mingot JM, Kostka S, Kraft R, Hartmann E, Görlich D (2001) Importin 13: a novel mediator of nuclear import and export. EMBO J, 20: 3685-3694

Mohr D, Frey S, Fischer T, Güttler T, Görlich D (2009) Characterisation of the passive permeability barrier of nuclear pore complexes. EMBO J, 28: 2541-2553

Monecke T, Güttler T, Neumann P, Dickmanns A, Görlich D, Ficner R (2009) Crystal structure of the nuclear export receptor CRM1 in complex with Snurportin1 and RanGTP. Science, 324: 1087-1091

Moore MS, Blobel G (1993) The GTP-binding protein Ran/TC4 is required for protein import into the nucleus. Nature, 365: 661-663

Moore MS, Blobel G (1994) Purification of a Ran-interacting protein that is required for protein import into the nucleus. Proc Natl Acad Sci U S A, 91: 10212-10216

Mosammaparast N, Jackson KR, Guo Y, Brame CJ, Shabanowitz J, Hunt DF, Pemberton LF (2001) Nuclear import of histone H2A and H2B is mediated by a network of karyopherins. J Cell Biol, 153: 251-262

Muhlhausser P, Muller EC, Otto A, Kutay U (2001) Multiple pathways contribute to nuclear import of core histones. EMBO Rep, 2: 690-696

Mullis, Faloona F, Scharf S, Saiki R, Horn G, Elich H (1986) Specific enzymatic amplification of DNA in vitro: the polymerase chain reaction. Cold Spring Harb Symp Quant Biol, 51 Pt 1: 263-273

Nachury MV, Weis K (1999) The direction of transport through the nuclear pore can be inverted. Proc Natl Acad Sci U S A, 96: 9622-9627

Nakajima T, Matsubayashi T, Kakimoto Y, Sano I (1971) Distribution of hypusine, N 6 -(4amino-2-hydroxybutyl)-2,6-diaminohexanoic acid, in mammalian organs. Biochim Biophys Acta, 252: 92-97

Nilsson J, Weis K, Kjems J (2002) The C-terminal extension of the small GTPase Ran is essential for defining the GDP-bound form. J Mol Biol, 318: 583-593 
Ohno M, Segref A, Bachi A, Wilm M, Mattaj IW (2000) PHAX, a mediator of U snRNA nuclear export whose activity is regulated by phosphorylation. Cell, 101: 187-198

Ohtsubo M, Okazaki H, Nishimoto T (1989) The RCC1 protein, a regulator for the onset of chromosome condensation locates in the nucleus and binds to DNA. J Cell Biol, 109: $1389-1397$

Okada C, Yamashita E, Lee SJ, Shibata S, Katahira J, Nakagawa A, Yoneda Y, Tsukihara $T$ (2009) A high-resolution structure of the pre-microRNA nuclear export machinery. Science, 326: 1275-1279

Ong SE, Blagoev B, Kratchmarova I, Kristensen DB, Steen H, Pandey A, Mann M (2002) Stable isotope labeling by amino acids in cell culture, SILAC, as a simple and accurate approach to expression proteomics. Mol Cell Proteomics, 1: 376-386

Ori A, Banterle N, Iskar M, Andres-Pons A, Escher C, Khanh Bui H, Sparks L, SolisMezarino V, Rinner O, Bork P, Lemke EA, Beck M (2013) Cell type-specific nuclear pores: a case in point for context-dependent stoichiometry of molecular machines. Mol Syst Biol, 9: 648

Paraskeva E, Izaurralde E, Bischoff FR, Huber J, Kutay U, Hartmann E, Luhrmann R, Görlich D (1999) CRM1-mediated recycling of snurportin 1 to the cytoplasm. J Cell Biol, 145: 255-264

Park JH, Wolff EC, Folk JE, Park MH (2003) Reversal of the deoxyhypusine synthesis reaction. Generation of spermidine or homospermidine from deoxyhypusine by deoxyhypusine synthase. J Biol Chem, 278: 32683-32691

Park JH, Wolff EC, Park MH (2011) Assay of deoxyhypusine hydroxylase activity. Methods Mol Biol, 720: 207-216

Park MH, Nishimura K, Zanelli CF, Valentini SR (2010) Functional significance of elF5A and its hypusine modification in eukaryotes. Amino Acids, 38: 491-500

Park MH, Wolff EC, Folk JE (1993) Hypusine: its post-translational formation in eukaryotic initiation factor $5 \mathrm{~A}$ and its potential role in cellular regulation. Biofactors, 4: 95-104

Partridge JR, Schwartz TU (2009) Crystallographic and biochemical analysis of the Ranbinding zinc finger domain. $\mathrm{J} \mathrm{Mol} \mathrm{Biol,} \mathrm{391:} \mathrm{375-389}$

Patel SS, Belmont BJ, Sante JM, Rexach MF (2007) Natively unfolded nucleoporins gate protein diffusion across the nuclear pore complex. Cell, 129: 83-96

Peat TS, Newman J, Waldo GS, Berendzen J, Terwilliger TC (1998) Structure of translation initiation factor $5 \mathrm{~A}$ from Pyrobaculum aerophilum at $1.75 \mathrm{~A}$ resolution. Structure, 6: 1207-1214

Peil L, Starosta AL, Virumae K, Atkinson GC, Tenson T, Remme J, Wilson DN (2012) Lys34 of translation elongation factor EF-P is hydroxylated by YfcM. Nat Chem Biol, 8: 695-697

Plafker SM, Macara IG (2000) Importin-11, a nuclear import receptor for the ubiquitinconjugating enzyme, UbcM2. EMBO J, 19: 5502-5513 
Plafker SM, Macara IG (2002) Ribosomal protein L12 uses a distinct nuclear import pathway mediated by importin 11. Mol Cell Biol, 22: 1266-1275

Pollard VW, Michael WM, Nakielny S, Siomi MC, Wang F, Dreyfuss G (1996) A novel receptor-mediated nuclear protein import pathway. Cell, 86: 985-994

Reichelt R, Holzenburg A, Buhle ELJ, Jarnik M, Engel A, Aebi U (1990) Correlation between structure and mass distribution of the nuclear pore complex and of distinct pore complex components. J Cell Biol, 110: 883-894

Reményi A, Lins K, Nissen LJ, Reinbold R, Schöler HR, Wilmanns M (2003) Crystal structure of a POU/HMG/DNA ternary complex suggests differential assembly of Oct4 and Sox2 on two enhancers. Genes Dev, 17: 2048-2059

Ribbeck K, Görlich D (2001) Kinetic analysis of translocation through nuclear pore complexes. EMBO J, 20: 1320-1330

Ribbeck K, Görlich D (2002) The permeability barrier of nuclear pore complexes appears to operate via hydrophobic exclusion. EMBO J, 21: 2664-2671

Ribbeck K, Kutay U, Paraskeva E, Görlich D (1999) The translocation of transportin-cargo complexes through nuclear pores is independent of both Ran and energy. Curr Biol, 9: 47-50

Ribbeck K, Lipowsky G, Kent HM, Stewart M, Görlich D (1998) NTF2 mediates nuclear import of Ran. EMBO J, 17: 6587-6598

Richards SA, Lounsbury KM, Macara IG (1995) The C terminus of the nuclear RAN/TC4 GTPase stabilizes the GDP-bound state and mediates interactions with RCC1, RAN-GAP, and HTF9A/RANBP1. J Biol Chem, 270: 14405-14411

Robbins J, Dilworth SM, Laskey RA, Dingwall C (1991) Two interdependent basic domains in nucleoplasmin nuclear targeting sequence: identification of a class of bipartite nuclear targeting sequence. Cell, 64: 615-623

Romacho T, Villalobos LA, Cercas E, Carraro R, Sanchez-Ferrer CF, Peiro C (2013) Visfatin as a novel mediator released by inflamed human endothelial cells. PLoS One, 8: e78283

Rongvaux A, Shea RJ, Mulks MH, Gigot D, Urbain J, Leo O, Andris F (2002) Pre-B-cell colony-enhancing factor, whose expression is up-regulated in activated lymphocytes, is a nicotinamide phosphoribosyltransferase, a cytosolic enzyme involved in NAD biosynthesis. Eur J Immunol, 32: 3225-3234

Rosorius O, Reichart B, Kratzer F, Heger P, Dabauvalle MC, Hauber J (1999) Nuclear pore localization and nucleocytoplasmic transport of elF-5A: evidence for direct interaction with the export receptor CRM1. J Cell Sci, 112: 2369-2380

Rout MP, Blobel G (1993) Isolation of the yeast nuclear pore complex. J Cell Biol, 123: 771-783 
Ruhl M, Himmelspach M, Bahr GM, Hammerschmid F, Jaksche H, Wolff B, Aschauer H, Farrington GK, Probst H, Bevec D, et A (1993) Eukaryotic initiation factor 5 A is a cellular target of the human immunodeficiency virus type 1 Rev activation domain mediating transactivation. J Cell Biol, 123: 1309-1320

Saini P, Eyler DE, Green R, Dever TE (2009) Hypusine-containing protein elF5A promotes translation elongation. Nature, 459: 118-121

Scheffzek K, Klebe C, Fritz-Wolf K, Kabsch W, Wittinghofer A (1995) Crystal structure of the nuclear Ras-related protein Ran in its GDP-bound form. Nature, 374: 378-381

Schneider TR, Sheldrick GM (2002) Substructure solution with SHELXD. Acta Crystallogr D Biol Crystallogr, 58: 1772-1779

Schnier J, Schwelberger HG, Smit-McBride Z, Kang HA, Hershey JW (1991) Translation initiation factor $5 \mathrm{~A}$ and its hypusine modification are essential for cell viability in the yeast Saccharomyces cerevisiae. Mol Cell Biol, 11: 3105-3114

Schwoebel ED, Talcott B, Cushman I, Moore MS (1998) Ran-dependent signal-mediated nuclear import does not require GTP hydrolysis by Ran. J Biol Chem, 273: 35170-35175

Seewald MJ, Korner C, Wittinghofer A, Vetter IR (2002) RanGAP mediates GTP hydrolysis without an arginine finger. Nature, 415: 662-666

Shevchenko A, Wilm M, Vorm O, Mann M (1996) Mass spectrometric sequencing of proteins silver-stained polyacrylamide gels. Anal Chem, 68: 850-858

Shi XP, Yin KC, Zimolo ZA, Stern AM, Waxman L (1996) The subcellular distribution of eukaryotic translation initiation factor, elF-5A, in cultured cells. Exp Cell Res, 225: 348356

Shiba T, Mizote H, Kaneko T, Nakajima T, Kakimoto Y (1971) Hypusine, a new amino acid occurring in bovine brain. Isolation and structural determination. Biochim Biophys Acta, 244: 523-531

Stuven T, Hartmann E, Görlich D (2003) Exportin 6: a novel nuclear export receptor that is specific for profilin.actin complexes. EMBO J, 22: 5928-5940

Sun D, Lee G, Lee JH, Kim HY, Rhee HW, Park SY, Kim KJ, Kim Y, Kim BY, Hong JI, Park C, Choy HE, Kim JH, Jeon YH, Chung J (2010) A metazoan ortholog of SpoT hydrolyzes ppGpp and functions in starvation responses. Nat Struct Mol Biol, 17: 11881194

Taylor CA, Sun Z, Cliche DO, Ming H, Eshaque B, Jin S, Hopkins MT, Thai B, Thompson JE (2007) Eukaryotic translation initiation factor 5A induces apoptosis in colon cancer cells and associates with the nucleus in response to tumour necrosis factor alpha signalling. Exp Cell Res, 313: 437-449

Teng YB, Ma XX, He YX, Jiang YL, Du J, Xiang C, Chen Y, Zhou CZ (2009) Crystal structure of Arabidopsis translation initiation factor elF-5A2. Proteins, 77: 736-740 
Terwilliger TC, Adams PD, Read RJ, McCoy AJ, Moriarty NW, Grosse-Kunstleve RW, Afonine PV, Zwart PH, Hung LW (2009) Decision-making in structure solution using Bayesian estimates of map quality: the PHENIX AutoSol wizard. Acta Crystallogr D Biol Crystallogr, 65: 582-601

Terwilliger TC, Grosse-Kunstleve RW, Afonine PV, Moriarty NW, Zwart PH, Hung LW, Read RJ, Adams PD (2008) Iterative model building, structure refinement and density modification with the PHENIX AutoBuild wizard. Acta Crystallogr D Biol Crystallogr, 64: $61-69$

Thomas F, Kutay U (2003) Biogenesis and nuclear export of ribosomal subunits in higher eukaryotes depend on the CRM1 export pathway. J Cell Sci, 116: 2409-2419

Thompson JE, Hopkins MT, Taylor C, Wang TW (2004) Regulation of senescence by eukaryotic translation initiation factor $5 \mathrm{~A}$ : implications for plant growth and development. Trends Plant Sci, 9: 174-179

Tong Y, Park I, Hong BS, Nedyalkova L, Tempel W, Park HW (2009) Crystal structure of human elF5A1: insight into functional similarity of human elF5A1 and elF5A2. Proteins, 75: $1040-1045$

Truant R, Cullen BR (1999) The arginine-rich domains present in human immunodeficiency virus type 1 Tat and Rev function as direct importin beta-dependent nuclear localization signals. Mol Cell Biol, 19: 1210-1217

Tsuchiya M, Ogawa H, Suzuki T, Sugiyama N, Haraguchi T, Hiraoka Y (2011) Exportin 4 interacts with Sox9 through the HMG Box and inhibits the DNA binding of Sox9. PLoS One, 6: e25694

Ude S, Lassak J, Starosta AL, Kraxenberger T, Wilson DN, Jung K (2013) Translation elongation factor EF-P alleviates ribosome stalling at polyproline stretches. Science, 339: 82-85

UniProt (2015) UniProt: a hub for protein information. Nucleic Acids Res, 43: D204-D212

Van den Wyngaert I, de Vries W, Kremer A, Neefs J, Verhasselt P, Luyten WH, Kass SU (2000) Cloning and characterization of human histone deacetylase 8. FEBS Lett, 478: 7783

Verreault A, Kaufman PD, Kobayashi R, Stillman B (1998) Nucleosomal DNA regulates the core-histone-binding subunit of the human Hat1 acetyltransferase. Curr Biol, 8: 96108

Vetter IR, Arndt A, Kutay U, Görlich D, Wittinghofer A (1999a) Structural view of the RanImportin beta interaction at 2.3 A resolution. Cell, 97: 635-646

Vetter IR, Nowak C, Nishimoto T, Kuhlmann J, Wittinghofer A (1999b) Structure of a Ranbinding domain complexed with Ran bound to a GTP analogue: implications for nuclear transport. Nature, 398: 39-46

Vetter IR, Wittinghofer A (2001) The guanine nucleotide-binding switch in three dimensions. Science, 294: 1299-1304 
Wang TW, Lu L, Wang D, Thompson JE (2001) Isolation and characterization of senescence-induced cDNAs encoding deoxyhypusine synthase and eucaryotic translation initiation factor 5A from tomato. J Biol Chem, 276: 17541-17549

Watson ML (1954) Pores in the mammalian nuclear membrane. Biochim Biophys Acta, 15: $475-479$

Weinmann L, Hock J, Ivacevic T, Ohrt T, Mutze J, Schwille P, Kremmer E, Benes V, Urlaub H, Meister G (2009) Importin 8 is a gene silencing factor that targets argonaute proteins to distinct mRNAs. Cell, 136: 496-507

Wen W, Meinkoth JL, Tsien RY, Taylor SS (1995) Identification of a signal for rapid export of proteins from the nucleus. Cell, 82: 463-473

Wild T, Horvath P, Wyler E, Widmann B, Badertscher L, Zemp I, Kozak K, Csucs G, Lund $E$, Kutay $U$ (2010) A protein inventory of human ribosome biogenesis reveals an essential function of exportin 5 in 60 S subunit export. PLoS Biol, 8: e1000522

Wirth M, Karaca S, Wenzel D, Ho L, Tishkoff D, Lombard DB, Verdin E, Urlaub H, Jedrusik-Bode M, Fischle W (2013) Mitochondrial SIRT4-type proteins in Caenorhabditis elegans and mammals interact with pyruvate carboxylase and other acetylated biotindependent carboxylases. Mitochondrion, 13: 705-720

Wolff EC, Lee SB, Park MH (2011) Assay of deoxyhypusine synthase activity. Methods Mol Biol, 720: 195-205

$\mathrm{Xu}$ A, Chen KY (2001) Hypusine is required for a sequence-specific interaction of eukaryotic initiation factor $5 \mathrm{~A}$ with postsystematic evolution of ligands by exponential enrichment RNA. J Biol Chem, 276: 2555-2561

Yanagisawa T, Sumida T, Ishii R, Takemoto C, Yokoyama S (2010) A paralog of lysyltRNA synthetase aminoacylates a conserved lysine residue in translation elongation factor P. Nat Struct Mol Biol, 17: 1136-1143

Yao M (2003) Crystal Structure of Hyperthermophilic Archaeal Initiation Factor 5A: A Homologue of Eukaryotic Initiation Factor 5A (elF-5A). Journal of Biochemistry, 133: 7581

Yi R, Qin Y, Macara IG, Cullen BR (2003) Exportin-5 mediates the nuclear export of premicroRNAs and short hairpin RNAs. Genes Dev, 17: 3011-3016

Yokoyama N, Hayashi N, Seki T, Pante N, Ohba T, Nishii K, Kuma K, Hayashida T, Miyata T, Aebi U, et A (1995) A giant nucleopore protein that binds Ran/TC4. Nature, 376: 184-188

Yoshida K, Blobel G (2001) The karyopherin Kap142p/Msn5p mediates nuclear import and nuclear export of different cargo proteins. J Cell Biol, 152: 729-740

Zanelli CF, Maragno AL, Gregio AP, Komili S, Pandolfi JR, Mestriner CA, Lustri WR, Valentini SR (2006) elF5A binds to translational machinery components and affects translation in yeast. Biochem Biophys Res Commun, 348: 1358-1366

Zanelli CF, Valentini SR (2007) Is there a role for elF5A in translation? Amino Acids, 33: 351-358 
Zender L, Xue W, Zuber J, Semighini CP, Krasnitz A, Ma B, Zender P, Kubicka S, Luk JM, Schirmacher P, McCombie WR, Wigler M, Hicks J, Hannon GJ, Powers S, Lowe SW (2008) An oncogenomics-based in vivo RNAi screen identifies tumor suppressors in liver cancer. Cell, 135: 852-864

Zuk D, Jacobson A (1998) A single amino acid substitution in yeast elF-5A results in mRNA stabilization. EMBO J, 17: 2914-2925 


\section{APPENDIX}

\begin{tabular}{|c|c|c|c|c|c|}
\hline O75436 & Q9UBQ0 & E5RJD8 & P62424 & P26373 & F8VQ10 \\
\hline Q8N584 & Q07960 & Q5JVF3 & Q9HCN4 & P50914 & F6QR24 \\
\hline Q96QK1 & Q92696 & C9JP52 & P84098 & P50542-4 & P30153 \\
\hline P10155 & P31947 & Q4G0F5 & P53611 & P62753 & \\
\hline
\end{tabular}

Table 7-1 Uniprot identifiers of the proteins that were significantly enriched in the presence of RanGTP

\begin{tabular}{|c|c|c|c|c|c|}
\hline Q53HV7 & Q9NTM9 & O60502 & P49915 & Q9H974 & P46060 \\
\hline Q16576 & P08397 & Q9BY41 & Q8WWN8 & Q9BXR0 & P49321-3 \\
\hline Q8N4P3 & O14929 & O75818 & Q96115 & Q96MB7 & O43813 \\
\hline P43487 & Q96SI1 & Q86W42 & O00571 & P43490 & P55060 \\
\hline
\end{tabular}

Table 7-2 Uniprot identifiers of the proteins that were significantly enriched in the absence of RanGTP 